\title{
Building-Integrated Photovoltaics: A Case Study
}

Gregory Kiss

Jennifer Kinkead

Kiss Cathcart Anders Architects, P.C.

Mahadev Raman

Ove Arup \& Partners New York

NREL Technical Monitor: Sheila Hayter

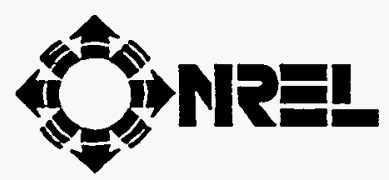

National Renewable Energy Laboratory 1617 Cole Boulevard Golden, Colorado 80401-3393

A national laboratory of the U.S. Department of Energy Managed by the Midwest Research Institute for the U.S. Department of Energy under Contract No. DE-AC36-83CH10093

March 1995 
This publication was reproduced from the best available camera-ready copy submitted by the subcontractor and received no editorial review at NREL.

\section{NOTICE}

This report was prepared as an account of work sponsored by an agency of the United States government. Neither the United States government nor any agency thereof, nor any of their employees, makes any warranty, express or implied, or assumes any legal liability or responsibility for the accuracy, completeness, or usefulness of any information, apparatus, product, or process disclosed, or represents that its use would not infringe privately owned rights. Reference herein to any specific commercial product, process, or service by trade name, trademark, manufacturer, or otherwise does not necessarily constitute or imply its endorsement, recommendation, or favoring by the United States government or any agency thereof. The views and opinions of authors expressed herein do not necessarily state or reflect those of the United States government or any agency thereof.

Available to DOE and DOE contractors from:

Office of Scientific and Technical Information (OSTI)

P.O. Box 62

Oak Ridge, TN 37831

Prices available by calling (615) 576-8401

Available to the public from:

National Technical Information Service (NTIS)

U.S. Department of Commerce

5285 Port Royal Road

Springfield, VA 22161

(703) $487-4650$ 


\section{DISCLAIMER}

Portions of this document may be illegible electronic image products. Images are produced from the best available original document. 


\section{CONTENTS}

Executive Summary $-i$

Introduction: - 1

Building Description: _-_

BIPV System Type - 8

BIPV System Options -_-_ 9

Energy Performance Evaluation _-_ 20

Cost Analysis - 26

Utility Rate Analysis - 42

Payback Analysis _-_____ 47

Conclusions - 63

Recommendations for Future Study -65

Appendices -67

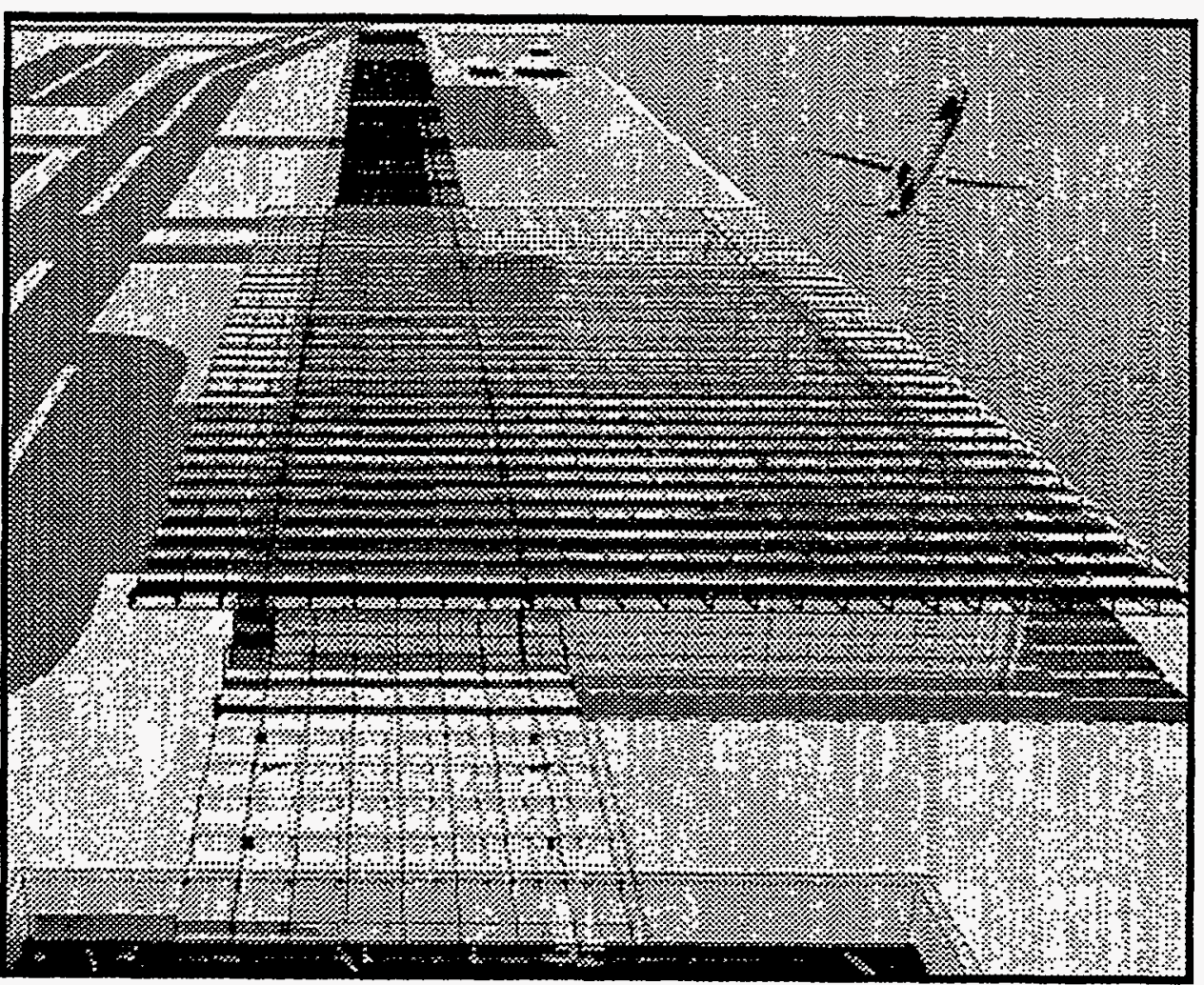

Fig. 1. Case study building: conference center and pedestrian canopy as seen from hotel roof.

Cover page: Conference center at main entry. 


\section{EXECUTIVE SUMMARY:}

\section{BACKGROUND}

In 1992, Kiss Cathcart Anders Architects performed a study for NREL on Building-Integrated Photovoltaics (BIPV) issues as seen from the perspective of the building community. That study included a broad overview of potential BIPV applications in commercial/institutional buildings. Wall, roof, and semidetached applications were illustrated, and some quantitative analysis of the effects of building geometry on PV output was performed. In general, the purpose of the study was to list major issues and potential applications; by it's nature it asked more questions than it answered.

\section{SCBOPE}

This second phase study was commissioned by NREL in 1994 to produce quantitative data on the performance of specific BIPV systems. High value-added applications for BIPV are targeted in medium to high-end commercial/institutional buildings. These building types should offer a good initial market for BIPV products.

\section{SYSTEMS EVALUATED}

In order to limit the number of variables only roof systems are evaluated. The energy performance, construction cost and simple payback for five different BIPV roof options are evaluated in each of six different locations around the US (Oakland, New York, Miami, Phoenix, Chicago and Cincinnati). The roof options include:

1. A single-glazed PV roof using glass-substrate PVs;

2. A double-glazed PV roof with insulating PV modules;

3. A ballasted roof-mounted system;

4. A sawtooth light monitor roof with indirect north daylighting;

5. A sawtooth roof with north light and active heat recovery.

\section{PV TECHNOLOGIES EVALUATED}

Each system was evaluated with three different PV technologies: Crystalline silicon at $\$ 4.40 / \mathrm{W}$ and $140 \mathrm{~W} / \mathrm{m} 2(13 \mathrm{~W} / \mathrm{sf})$, amorphous silicon at $\$ 3 / \mathrm{W}$ and $52 \mathrm{~W} / \mathrm{m} 2(4.8 \mathrm{~W} / \mathrm{sf})$, and advanced thin films (for future applications) at $\$ 2 / \mathrm{W}$ 
and $108 \mathrm{~W} / \mathrm{m} 2(10 \mathrm{~W} / \mathrm{sf})$. The first two technologies are currently available; the costs and efficiencies of the third are projections for the future.

\section{BIPV SYSTEM COSTS AND ENERGY PERFORMANCE}

Construction costs and energy benefits of all BIPV roof systems are compared with a reference design (a standard flat, opaque, insulating roof). The reference design has a cost basis of zero; any additional structure, construction materials, PV modules or electrical systems are counted as costs. If the BIPV roof option displaces any of the materials used in the reference design (ie, roof membrane, insulation or metal roof deck), the cost of that material is subtracted from the cost of the BIPV option.

The energy benefits of each BIPV roof system are compared with the reference roof to determine the net effect of each system on building energy consumption. In several of the BIPV options the PV module itself is the building roof; in these cases energy savings or losses due to heat or light transmission through the module are always included in the anlayses. In some of the BIPV systems the PV modules are not integral to daylight or thermal performance; in these cases payback is also analyzed two ways:

1 for the complete system, including all construction costs and all energy benefits, and

2 for the PV system only, including only PV-related costs (modules, wiring, inverters) and using only PV electricity as income.

\section{LOCATIONS EVALUATED}

Oakland, New York, Miami, Phoenix, Chicago and Cincinnati represent a wide range of climate types from temperate cold to subtropical, and an equally wide range of utility rates and construction costs.

\section{UTILITY RATE ANALYSIS}

In each city an applicable utility rate is selected which seems likely to provide the greatest value for power offset by the PV system. Plans with high time of use charges and low demand charges are chosen where available. The actual value of power offset by the PV system is calculated by multiplying the hourly PV output for each day of each month by the rate prevailing at each hour. The resulting total dollar figure is divided by total electrical production for the year to give an average dollar $/ \mathrm{kWh}$ electrical rate. 


\section{PAYBACK}

In the case of the full system analysis (including collateral energy effects), the value of the total energy difference between each option and the base case was divided into the construction cost premium to establish a simple payback. For the PV-only analysis, the value of the electricity generated by the system was divided into the cost of the PV system only. The payback calculations do not take into account tax benefits.

\section{CONCLUSIONS}

- Payback periods range from 14.5 years and up. Of the six locations in the analysis, the best environment for BIPV systems is Oakland, where insolation is good, the climate is mild and energy costs are high. The worst case is Cincinnati, where insolation, climate and energy costs are all less favorable.

-Systems with payback periods of approximately twenty years according to this study) can be cost-effective today. Tax benefits, which are not included in these calculations, can cut payback times significantly, bringing them into the tenyear range. Although the mass market usually requires payback of less than five years, institutions with longer planning horizons can find these applications economical now.

-No one PV technology is most cost effective in all cases. The study indicates that either high efficiency, high cost PVs like single crystal or low efficiency, low cost PVs like amorphous silicon can perform better in the right circumstances. The former technology (high $\mathrm{W} / \mathrm{m} 2$ ) is less affected by high area-related balance of system costs, such as glazing framing systems; the latter technology (low $\$ / \mathrm{m} 2$ ) benefits more from credits from avoided construction materials, such as atrium glass.

- No one BIPV roof system is most cost effective in all cases. Applications offering significant material credits, such as skylights or atriums, usually give the best return. In some cases, systems that combine daylight and thermal benefits with PV can be cost effective even if they are not displacing expensive construction materials. 
- This study did not attempt to identify the best possible application for BIPV. Shorter payback periods may be possible with other BIPV systems which offer higher material credits, such as curtain walls, or in other locations with higher utility rates or more favorable climates. It is worth repeating, however, that within the scope of this study cost-effective BIPV applications exist today. 


\section{INTRODUCTION:}

In January 1993 Kiss Cathcart Anders Architects (Gregory Kiss and Jennifer Kinkead, investigators) completed a study for the National Renewable Energy Laboratory entitled "Building-Integrated Photovoltaics". Based on the premise that there are many economic advantages to integrating Photovoltaics into building construction, the report was a broad survey of the issues involved in integrating Photovoltaics (PVs) into buildings, seen from the perspective of the building industry. The market for building-integrated products was (and still is) in its infancy. Although advances in PV technologies have made many new applications possible, development of products specifically designed for building use has barely begun.

One of the barriers to the creation of a Building-Integrated Photovoltaic (BIPV) industry is a lack of knowledge about how PV-building products will perform in place, as construction materials as well as PV devices. At present-there are few built BIPV projects in the United States from which to gain experience. Most of these have been residential, many of them retrofits to existing houses. In Europe many more BIPV projects have been built, including many residences and a number of advanced, high-visibility commercial and institutional projects.

Our 1993 report concentrated on commercial and institutional building applications because we believe that these building types are the highest value-added applications for PVs, and will therefore be a viable early (and long term) market for BIPV products. In commercial and institutional buildings BIPV materials can be used to replace conventional building materials which cost as much as or more than the PVs; these buildings often have sophisticated envelope and mechanical systems which offer the potential to capture additional daylighting and thermal energy benefits; and their load profiles are well matched to peak sun (and peak electricity rates). The previous report surveyed these and many other issues from the point of view of the design community and the building industry, and while it included a number of schematic system designs and some quantitative analysis, by its nature it raised more questions than it answered. 
This report is intended to help answer some of these questions, particularly:

- How would a BIPV system in a medium-sized institutional building perform in terms of the thermodynamic behavior of the building envelope, as well as in terms of electrical output?

- How will these systems be constructed?

- How much will they cost and what will the payback be?

A building is a combination of many complex systems: structural, mechanical, electrical, and others. Changes to the parameters of one system affect the others. An assessment of the performance of a BIPV system as an element of a building skin therefore requires a multidisciplinary approach. We collaborated on this study with Mahadev Raman and Ross Clarke, mechanical and structural engineers respectively, at Ove Arup and Partners New York, a building engineering firm. We began by creating a hypothetical building program, then selecting a basic type of BIPV system (roof or wall) to develop for design and analysis. In setting these initial criteria we relied on our subjective judgment, since an exhaustive analysis of possible building types, programs and sites was beyond the scope of this study. In any case we were not attempting to select the optimum project for PV integration; we were interested in a building that would be in the mid- to high-end of the cost and performance range, and that would have aspects that would be applicable to other projects.

The building type was selected according to the following criteria: that it be a mid-size commercial project in the $\$ 10-20$ million construction cost range; that it operate primarily during daylight hours; that it be public or semi-public to ensure good visibility for the PV systems; that it have a medium to high level of material finish; and that it be suitable for a BIPV system that could develop significant corollary energy benefits or displace significant construction material costs, or both. Given these parameters, we selected a medium sized conference and convention center as the program.

Among the range of PV-integration options for a convention center, we chose to study integration at the roof of the main exhibition hall. Rooftop BIPV systems offer opportunities for good orientation and collateral energy benefits. In addition, the one-to-one ratio between roof and floor area enabled us to confine 
our analysis to a typical square meter of interior space for energy demand and production.

While the building was designed in its entirety in English units to establish a scale for the project and to provide peak electrical demand numbers for use in utility rate analysis, all other analyses, including thermal performance, construction cost and payback, were performed on a square meter basis. 


\section{BUILDING DESCRIPTION:}

The case study building is a conference center designed to support mediumsized conferences and exhibitions for 300 to 500 persons at a time. The total building floor area is $6,700 \mathrm{~m} 2$ gross $(72,000 \mathrm{sf})$.

Note that the site diagram (Fig 2) shows a larger program which includes a hotel and pedestrian/parking structure, all PV-integrated. This case study focuses exclusively on the conference center. The renderings illustrating the report (cover page, Figs. 1, 3, 6, 30) depict a sawtooth roof system with light monitors. The sawtooth roof system is illustrated to give a general sense of the the architectural quality of a combined PV/daylight structure. The other BIPV roof systems were also analyzed and are illustrated with diagrams, sections and detail perspectives in the BIPV Options and Cost Analysis sections.

For the purpose of the energy balance analysis, occupancy is projected from 9am-7pm daily. Standard internal environmental criteria apply for temperature, humidity and lighting (Appendix A outlines these criteria and other building load assumptions in more detail). The building is projected to have a peak demand of $\leq 500 \mathrm{~kW}$ and is thus considered a medium-scale commercial utility user. 


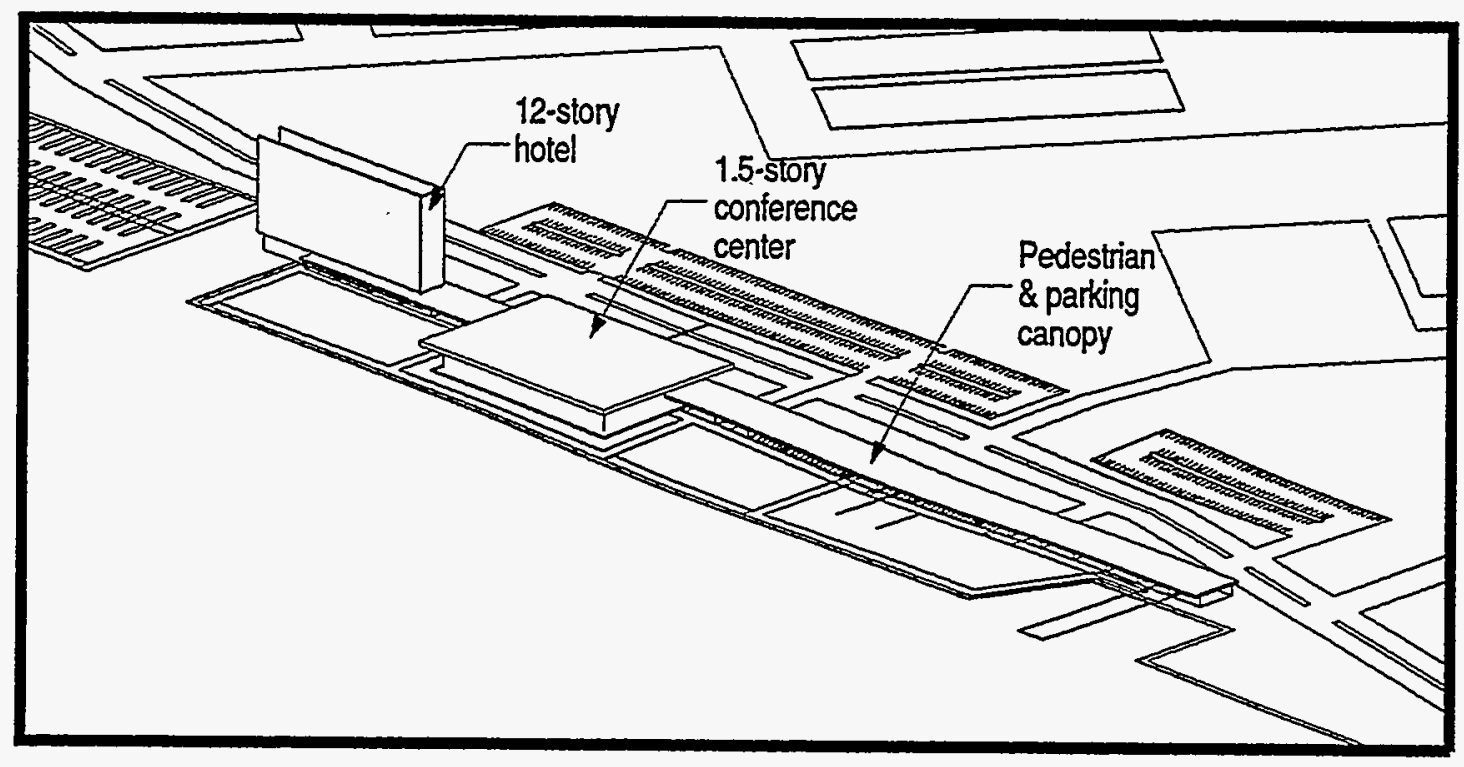

Fig 2. Case study building and surrounding site.

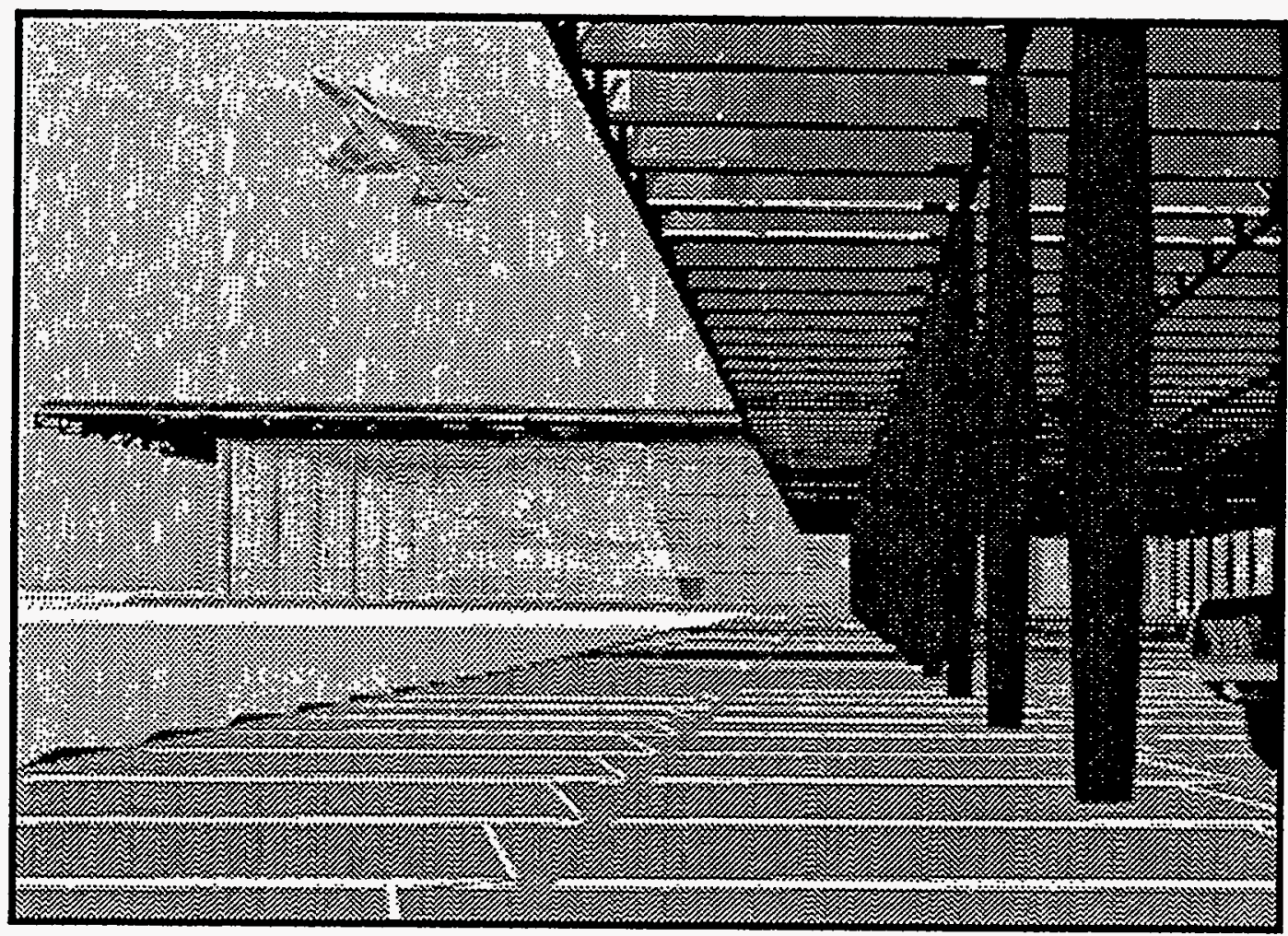

Fig 3. Conference center as seen from below parking canopy. 


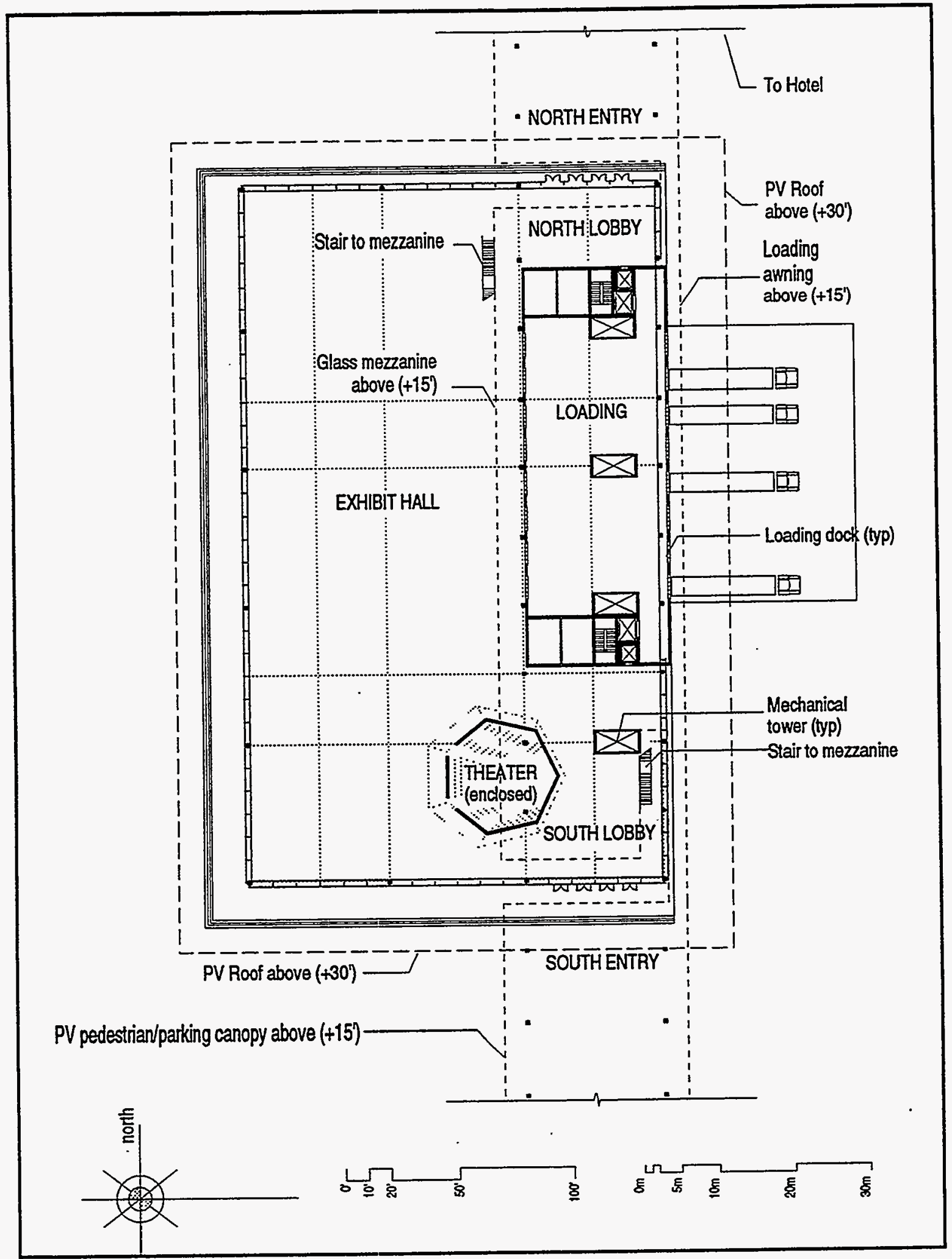

Fig 4. Conference center ground floor plon. 


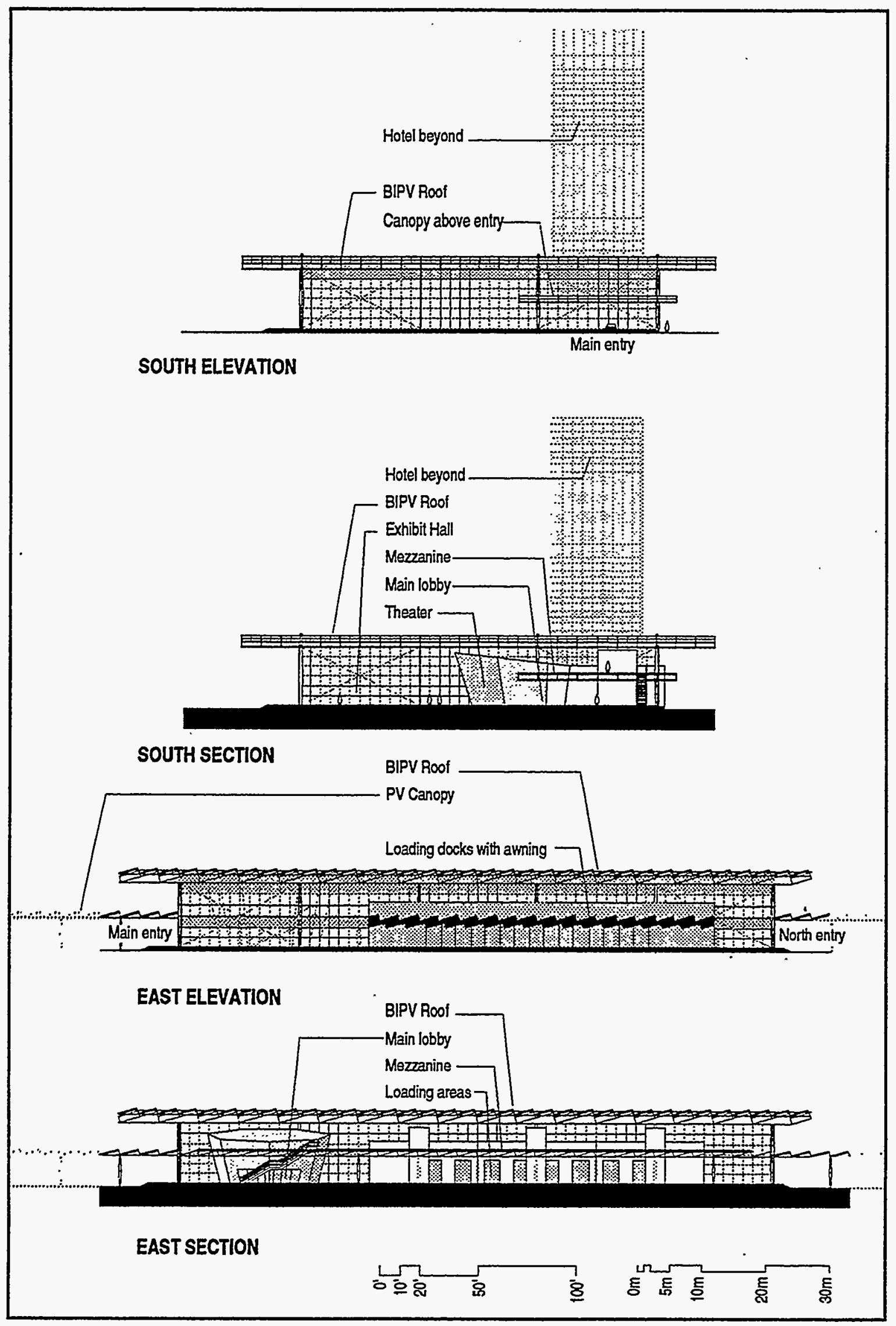

Fig 5. Conference center elevations and sections. 


\section{BIPV SYSTEM TYPE}

The external surfaces of a building have many functions: they keep out the weather, provide security and keep heat out (or in). A BIPV system adds another role to this program: generating power. A building surface can usually be classified as a roof or a wall, with significant differences in function, construction, and thermal and solar loadings. For the sake of clarity, we chose to design and analyze exclusively a roof-type BIPV system.

The choice of the conference center program influenced this decision in favor of a roof system. BIPV wall systems generally have a higher potential than roofs to displace costly building materials, but they suffer PV output losses due to relatively poor orientation. There is also relatively little wall area in most conference and convention centers: they are usually one story structures featuring a large exhibition and meeting space, creating a high roof to floor area ratio. In this case we felt that a roof system would have good PV performance, good potential for collateral energy benefits, and important significance as an architectural element. A roof system also has the advantage of being easy to analyze on a per square meter basis, making the results applicable to a wide range of building sizes.

A conference center is frequently built in conjunction with other facilities, and our site diagram (Fig 2) shows an adjacent hotel. The hotel is articulated as a tall thin slab, with maximum east/west exposure, ideal for BIPV curtain wall applications. Although we did not analyze the hotel in detail for this study, it plays a role in the economic analyses of the conference center. Since the hotel would not be energy self-sufficient from its own PV skin, it serves as a built-in customer for any surplus power produced by the conference center. As a rule, it generally does not pay to produce a surplus of PV power, since present policy in the US provides a low return on power sold back to the utilities. Our payback analyses assume the full retail peak cost of electricity is avoided by the PV system at all times.

Once the building program and site were selected, the project was designed to a level of detail sufficient for the schematic thermal and cost analyses which follow. 


\section{BIPV SYSTEM OPTIONS}

We identified five BIPV roof systems and analyzed each to determine its overall impact on the building's energy balance. A conventional flat opaque roof design served as the basis for comparison. This analysis was performed in six different climates. Construction cost estimates of each system were prepared, and the simple payback was calculated by dividing the construction cost premium by energy savings multiplied by local electrical rate.

The principal function of a roof is to keep out the precipitation and, secondarily, to moderate radiation (heat and light) between the interior and exterior environment. By selectively allowing light in and heat in or out, the roof can reduce building loads by supplementing or replacing lighting and heating/cooling systems. We attempted to find roof configurations that served as many functions as possible at a minimum construction cost premium. Since PVs should capture as much direct sunlight as possible, they can easily be combined with clerestory glazing which captures only indirect light. PVs on glass substrates (the majority of large-area PV modules suitable for building integration are on glass substrates) can also be made semi-transparent, and can be designed to pass some direct sunlight into a space while producing electricity. The heat that PVs create heat as they convert sunlight to electricity can be used or discarded as required.

The five BIPV roof configurations included two using direct-transmission semitransparent $P V$ roofs, two in a roof monitor (sawtooth) configuration with indirect light gain, and one flat opaque PV roof-mounted array. The latter system was based on the standard flat roof (the reference design for energy and cost calculations), with a gravity-mounted (ballasted) PV array above the roof membrane. This system is roof-mounted as opposed to roof-integrated, in that the PVs serve no function as a construction material: they do not form a weathering surface or serve other architectural functions.

In all the BIPV systems the PVs had the same orientation: $15^{\circ}$ tilt, azimuth southwest (see Utility Rate Analysis for a discussion of the effects of orientation on PV output). 


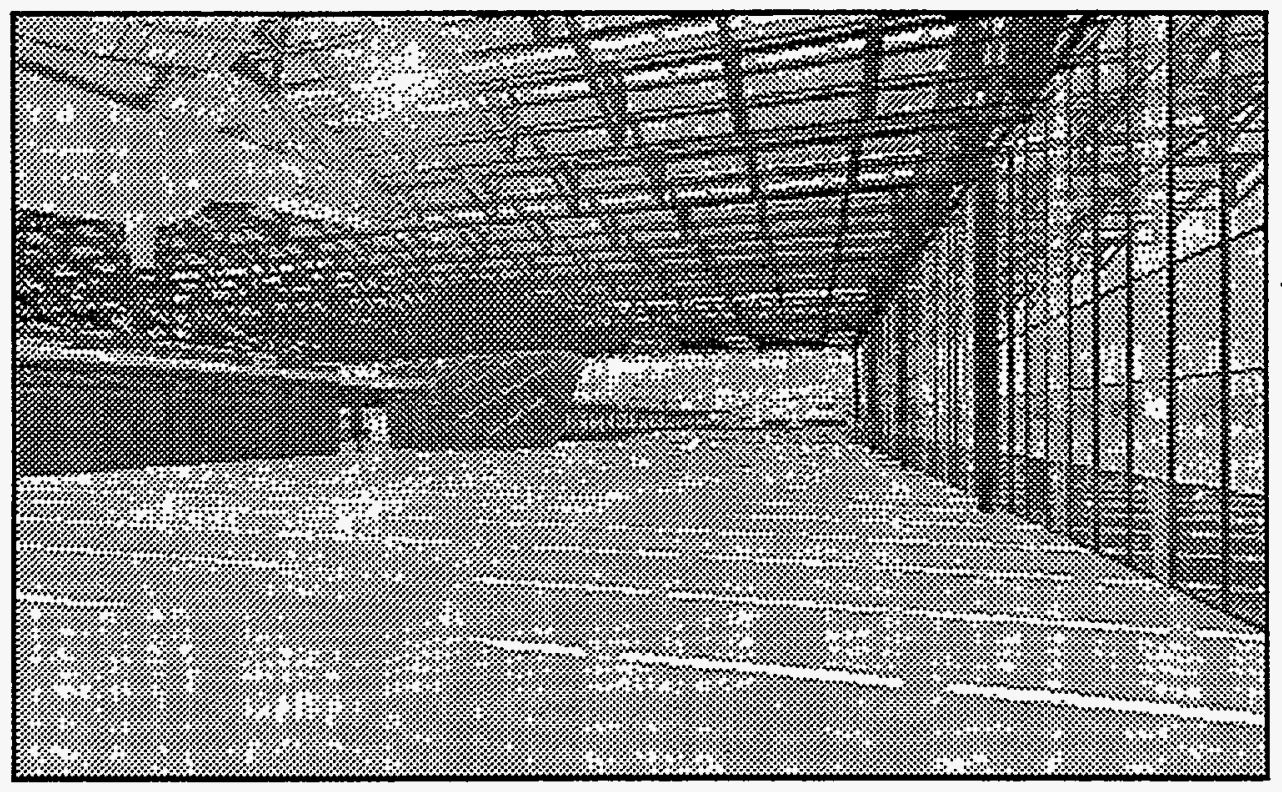

Fig 6. Conference center exhibition hall. 


\section{OPTION 1: SEMI-TRANSPARENT PV ROOF}

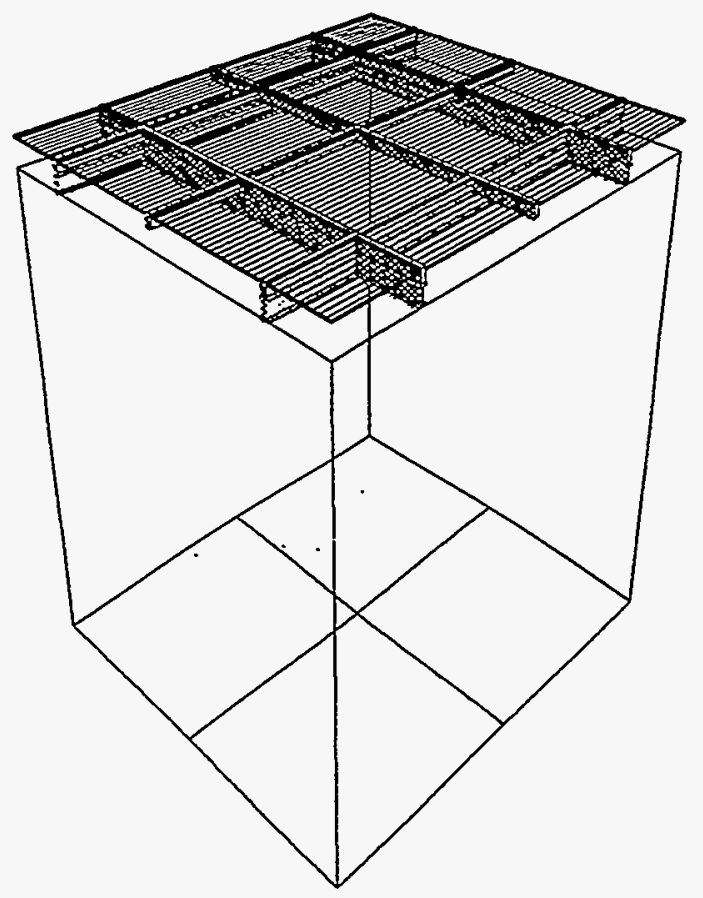

Fig 7. Option I partial roof illustration. Note: all PV output analyzed at $15^{\circ}$ tilt.

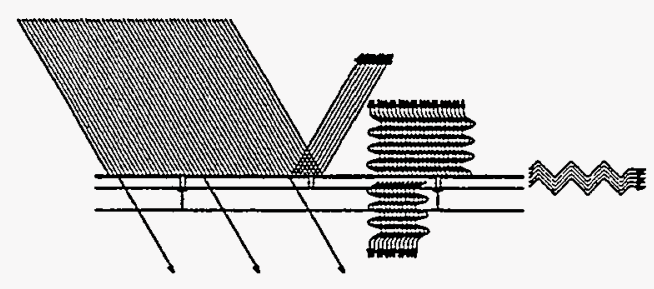

Fig 8. Option 1 energy balance diagram (see Fig 21 for labels and quantities).

\section{SSUMMARY:}

- Poor thermal performance results in building energy consumption more than twice that of better insulated alternatives.

- Wide extremes of building loads requires oversized HVAC systems, increasing capital cost.
This system is similar to large-area skylights, with semi-transparent modules in aluminum frames. Existing thin film technologies can produce glassencapsulated modules that are $\approx 5 \%$ transparent. With additional scribing or patterning of the modules the transparency can be increased to $15 \%$ or $20 \%$ or beyond, although the module efficiency decreases in proportion to the transparent area. Other existing technologies where single crystal or polycrystalline cells are laminated between glass sheets also offer flexible degrees of transparency. A $5 \%$ transparent roof over a large area is sufficient for most daylighting purposes: bright sunlight is approximately 100,000 lux, which will transmit 5,000 lux to the interior, much more than the standard artificial lighting range of 500-1,000 lux. On a cloudy day 10,000 lux exterior will be transmitted through as 500 lux, at the low end of standard illumination levels.

Standard laminated glass-to-glass modules have poor thermal performance. A 5\% transparent module has a low shading coefficient (a measure of direct radiation transmitted) but a high U-value (a measure of thermal conductivity). Because of the high U-value, building energy costs are high. There are wide extremes of heating and cooling require- . ments, which affects operating costs and requires an oversized HVAC system to handle the loads. The direct daylighting scheme may have a tendency to overlight the space on sunny days and underlight it on cloudy days, when supplemental artificial lighting may be required. 


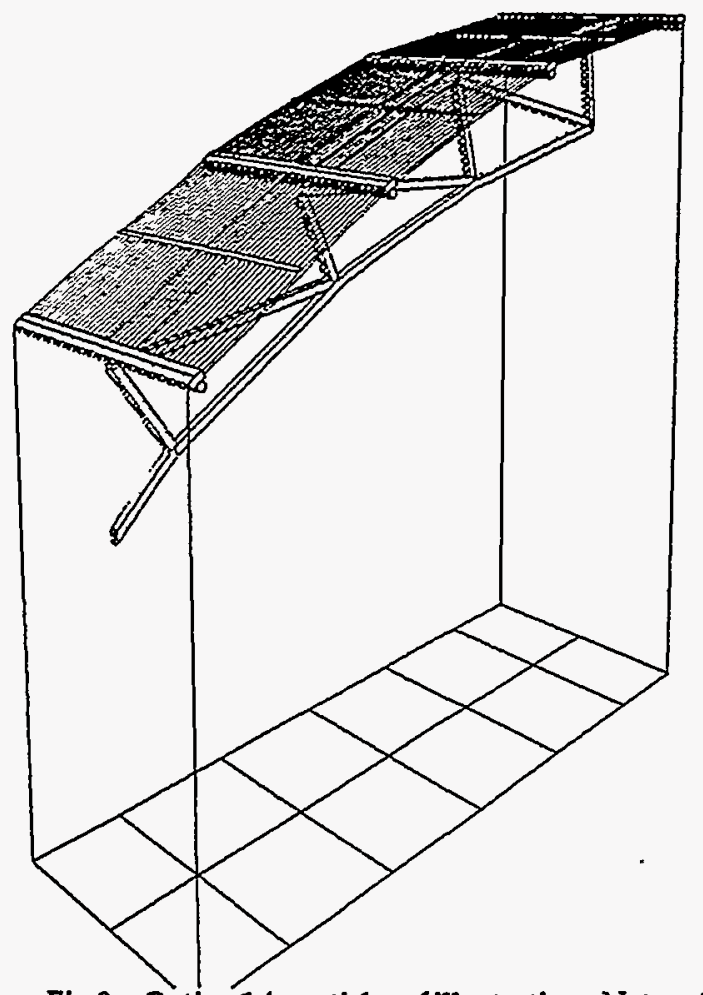

Fig 9. Option $1 A$ partial roof illustration.. Note: all PV output analyzed at $15^{\circ}$ tilt.

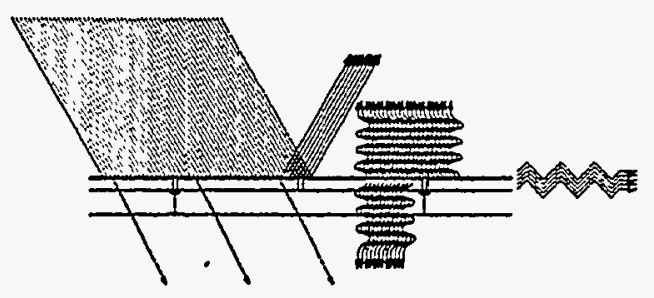

Fig 10. Option $1 A$ energy balance diagram (see Fig 21 for labels and quantities).
OPTION 1A: SEMI-TRANSPARENT SINGLE GLAZED PV ATRIUM

This option is similar to Option 1 in PV output and daylight and thermal performance. For cost estimation, this system is compared to the base case of an atrium roof, where skylight framing, laminated glass, and exposed architectural support structure are present.

When this system is considered for applications where an atrium exists or is planned, the daylight and thermal properties are not an issue- atria are often semiconditioned spaces - and a material credit is given for the cost of displacing tinted laminated atrium glass.

\section{SUMMARY:}

- Lowest installed cost due to simple installation and good material credit.

- Poor thermal performance may not be a factor in some climates or in cases where atrium space is not fully conditioned 


\section{OPTION 2: SEMI-TRANSPARENT DOUBLE} GLAZED PV ROOF

This system is similar to Option 1, with the substitution of high-performance double glazed PV lites for the single glazed versions. The laminated PV module is bonded to an inner lite with a spacer $13 \mathrm{~mm}\left(0.5^{\prime \prime}\right)$ thick. The cavity can be air or argon filled, and a low-E coating can be applied to the second lite to further improve the energy performance.

This option has much better energy performance than Option 1, and better overall energy performance than for an opaque insulated roof due to the daylighting contributions. (see Energy Performance Evaluations, p. 19)

Fig 11. Option 2 partial roof illustration. Note all PV output analyzed at $15^{\circ}$ tilt.

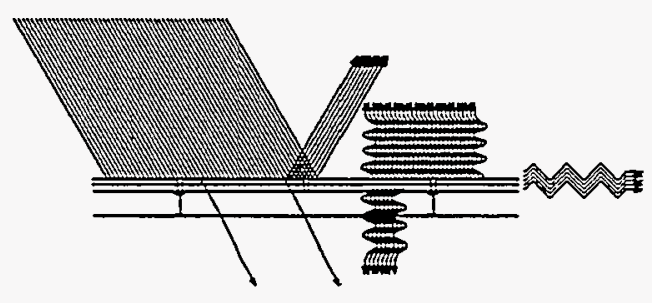

Fig 12. Option 2 energy balance diagram (see Fig 21 for labels and quantities).
Direct light transmittance is somewhat lower for this scheme than for Option 1, since the additional glazing layer absorbs more light. However, the potential for over-lighting and under-lighting, and the reliance on artificial lighting for cloudy days in winter, are still present in this scheme.

\section{SUMMARY:}

- Good overall energy performance despite low thermal insulation value, due to daylighting contribution.

- Tendency to over-and under-light space below; supplemental lighting may be required on cloudy days.

- Insulated Glazing PV unit not yet available as product; potentially expensive. 


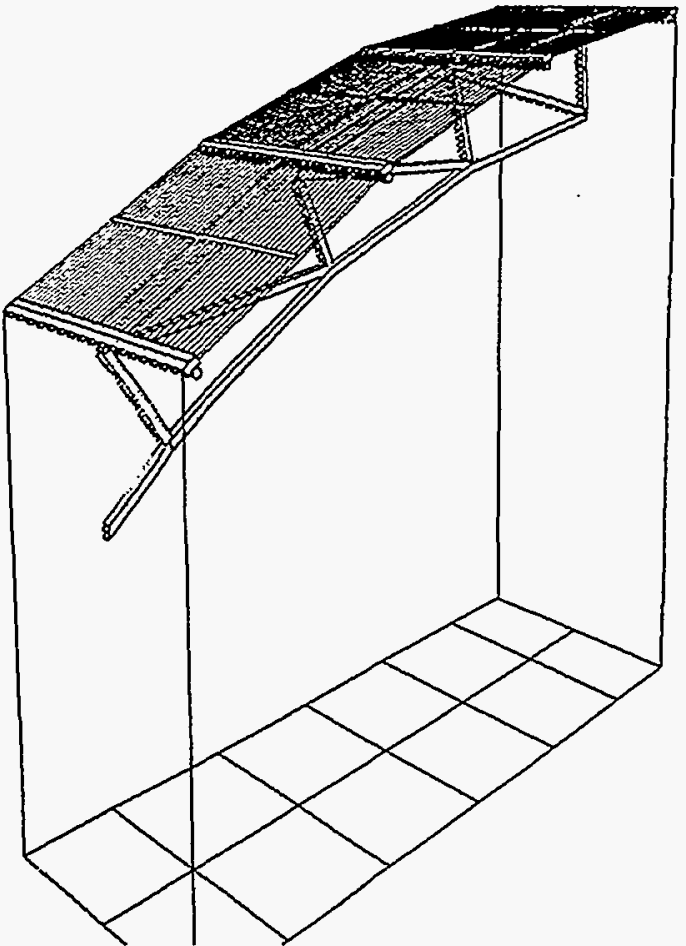

Fig 13. Option 2a partial roofillustration.

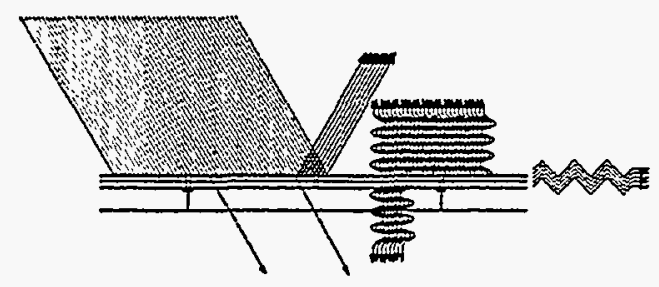

Fig 14. Option $2 a$ energy balance diagram (see Fig 21 for labels and quantities).

\section{OPTION 2A: SEMI-TRANSPARENT DOUBLE GLAZED PV ATRIUM}

This option is similar to Option 2, for applications where an atrium exists or is planned. For cost estimation, this system is compared to the base case of an atrium roof, where skylight framing, laminated double-glazing, and exposed architectural support structure are present.

It is important to note that an insulated PV-glazed atrium will not provide as much daylight as conventional glazing, which is roughly comparable to unfiltered sunlight. The light levels in this kind of space would be more like a shaded outdoor space, which would be more than adequate for most purposes and indeed would be more comfortable than a conventional atrium for extended occupation. The amount of light admitted to a space can be controlled by mixing PV with conventional glazing or by specifying PV with a custom transparency. In the cost analysis (see following section) a relatively high material credit is given for the cost of displacing insulated atrium safety glass.

\section{SUMMARY:}

- Similar to Option 2, with a higher material credit.

- Replaces a traditional atrium roof.

- Not as much daylight as traditional atriums would be a more comfortable environment to occupy for long periods.

- Much more energy efficient than a traditional atrium roof. 


\section{OPTION 3: FLAT BALLASTED ROOF ARRAY}

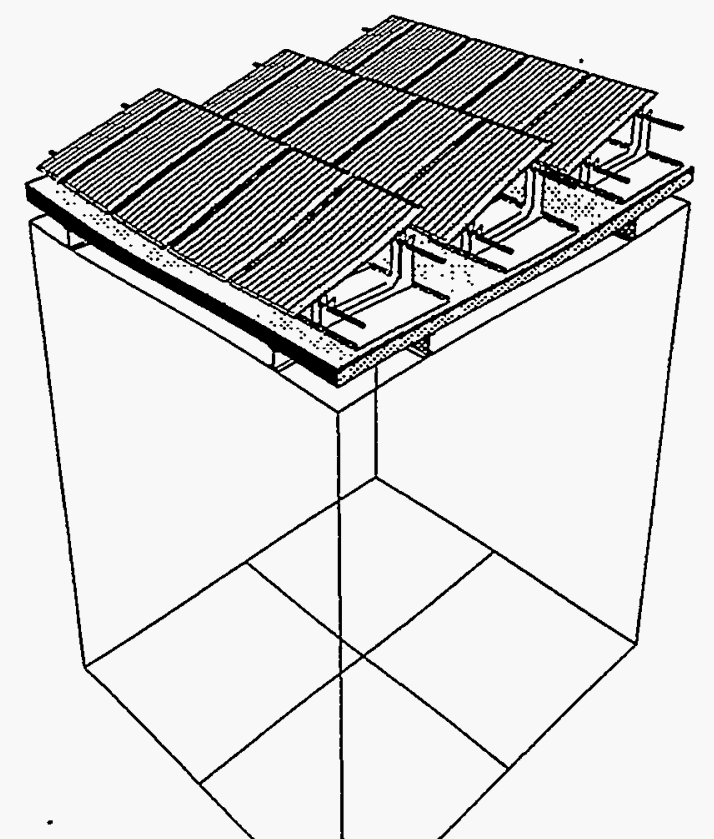

Fig 15. Option 3 partial roof illustration.

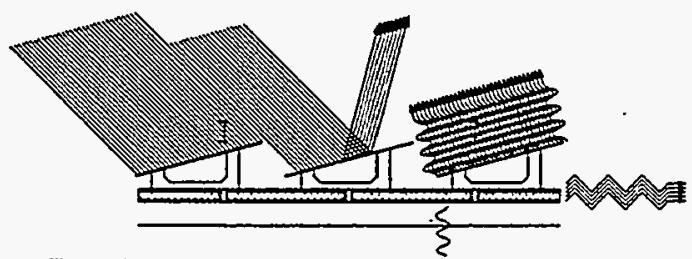

Fig 16. Option 3 energy balance diagram (see Fig 21 for labels and quantities).

\section{SUMMARY:}

- Simple installation, avoids roof penetrations.

- Potentially low cost.

- No material credits or daylight/thermal benefits.

- May have limited applicability due to code problems.
This is a conventional flat commercial roof: steel structure, steel deck, rigid insulation (Metric U 0.3; english R28.4), membrane roofing, with PVs installed on ballasted trays above the membrane.

This system is simple to install and does not penetrate the roof membrane, but several limitations exist:

-Seismic codes may not permit a system that is not physically restrained from lateral movement.

-The weight of the system (in excess of 10-15psf over the PV area) exceeds the load bearing capacity of many commercial roofs, which are typically designed to minimum structural criteria.

The costs of the structure and installation should be low, but the system does not gain any material credits by displacing other construction materials, and has no daylighting and limited thermal energy benefits: the value of the shading to the reference roof design is approximately $U 0.2$, which yields energy reductions from $0.45-1.2 \mathrm{kWh} / \mathrm{m} 2 / \mathrm{yr}(\$ 0.08$ $-0.11 / \mathrm{m} 2 / \mathrm{yr})$.

Due to the ease of installation and minimal disruption to the building envelope, this system is well suited for retrofit applications, although rooftop equipment, parapets and other obstructions may significantly limit available rooftop areas. 


\section{OPTION 4: PV ROOF MONITORS}

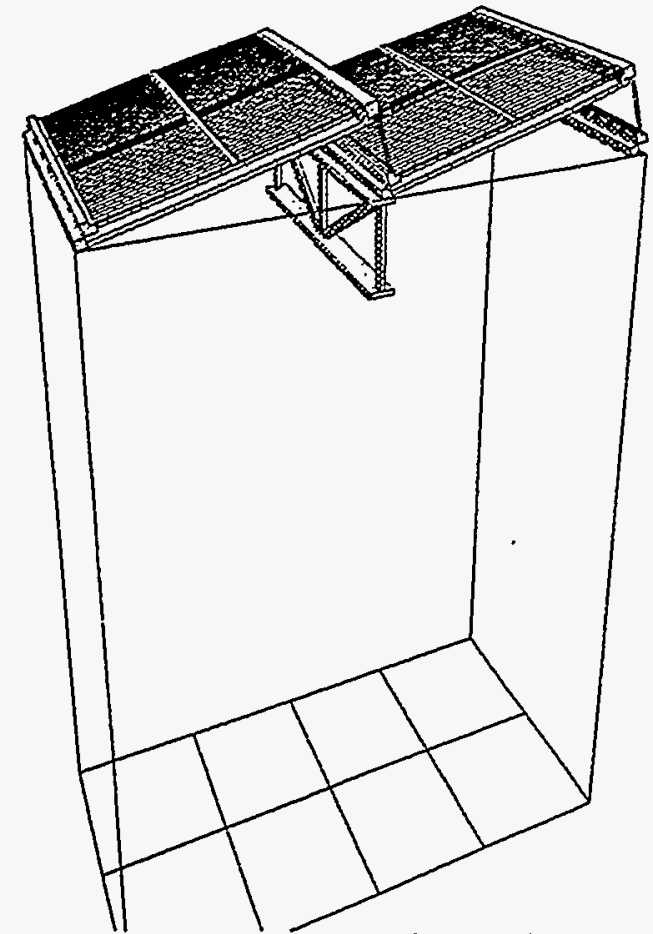

Fig 17. Option 4 partial roof illustration.

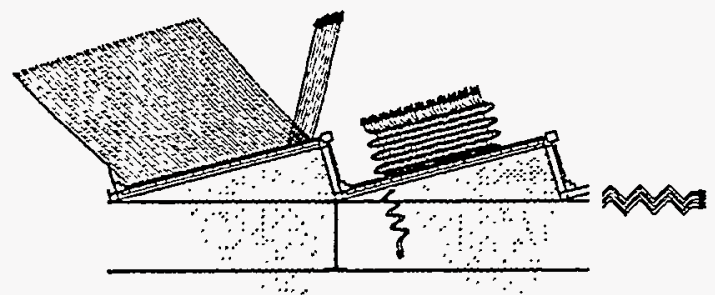

Fig 18. Option 4 energy balance diagram (see Fig 21 for labels and quantities).

\section{SUUMMARY:}

- Medium to high construction cost.

- Very good energy performance.

- Tilt on PVs and clerestories provides reliable drainage and helps keep modules clean.
This is a variation of the traditional industrial light monitor sawtooth roof system. The benefits of indirect natural light have been apparent to artists and industrialists alike: diffuse, variable, and free, it has psychological benefits that go beyond the light provided.

The north-facing clerestory glazing is complemented by PVs on the opaque south facing side of the roof monitor. The increased slope given the PVs when compared to other large area flat roofs has benefits because of higher output per unit area of PV, and also has better drainage and less dirt buildup. The sawtooth can also create problems with snow accumulation and ponding of water: these issues must be carefully studied in each case.

The PV can be insulated with inexpensive materials, giving the roof system as a whole a higher insulating value than any transparent system could have. Opaque or glass substrate PVs can be used. For glass modules, a shingled mounting method that relies partly on overlaps and flashing techniques might be used instead of conventional pressure plate systems; or other substrates such as plastic or sheet metal can be used instead. Interlocking plastic shingle sub-substrate systems have been developed by a number of manufacturers, although . these add an extra element to the product. Metal substrate PVs can be used directly as a building material; like glass, sheet metal is already a building material. Formed into pans, modules of this type can be used as sheet metal for standing seam, flat seam or batten seam roofing. 


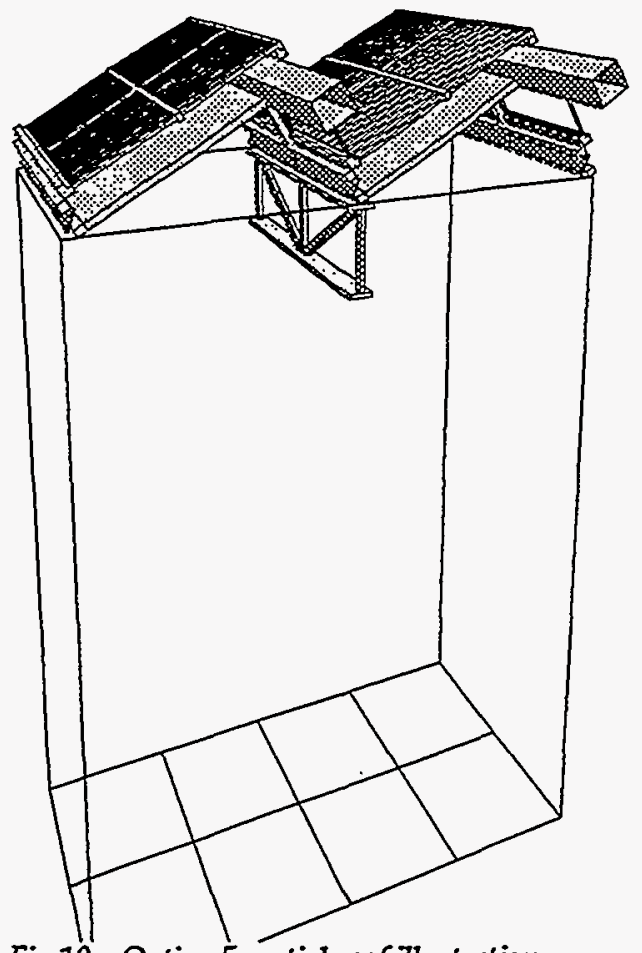

Fig 19. Option 5 partial roof illustration.

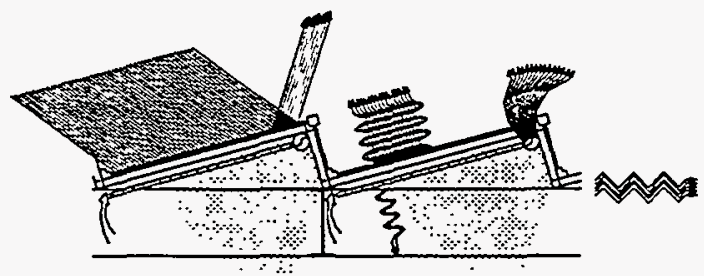

Fig 20. Option 5 energy balance diagram (see Fig 21 for labeis and quantities).

\section{SUMMARY:}

- Best energy performance.

- System is most effective if all the heat captured cen be used without requiring long-term thermal storage.

- High construction costs.

- Active systems require maintenance and repair, increasing life cycle cost.
OPTION 5: PV ROOF MONITORS WITH ACTIVE HEAT RECOVERY

This variation of Option 4 has the most ambitious energy agenda of all the schemes: recovery of as much of the PV heat buildup as possible via a dedicated ventilation system. This entails constructing a second insulated layer below the single-glazed PVs. The airspace within is vented at the top into a duct which feeds into the building HVAC system, or can be vented to the outside when there is no need for the heat. The heat removal also cools the PVs, which decreases thermal degradation of PV output, and reduces thermal stresses on the modules.

The biggest drawback with this system approach is the limited need for heating in most commercial buildings in the US. In Oakland, the system yields $20 \mathrm{x}$ the total heating requirement of the building. Even in Chicago, the coldest climate we evaluated, the system produces $3 x$ the total building heat requirements. If a use were available for all the heat collected, (for an industrial process use or pool water heating, for example), thermal storage might be required to make the heat available at times other than peak sun.

A solution to this problem would be to apply this system to only as much of the building's roof surface as necessary to generate a usable amount of heat. It could be easily combined with an Option 4 roof for this purpose. 
Fig 21. Energy Balance Diagram Summary.
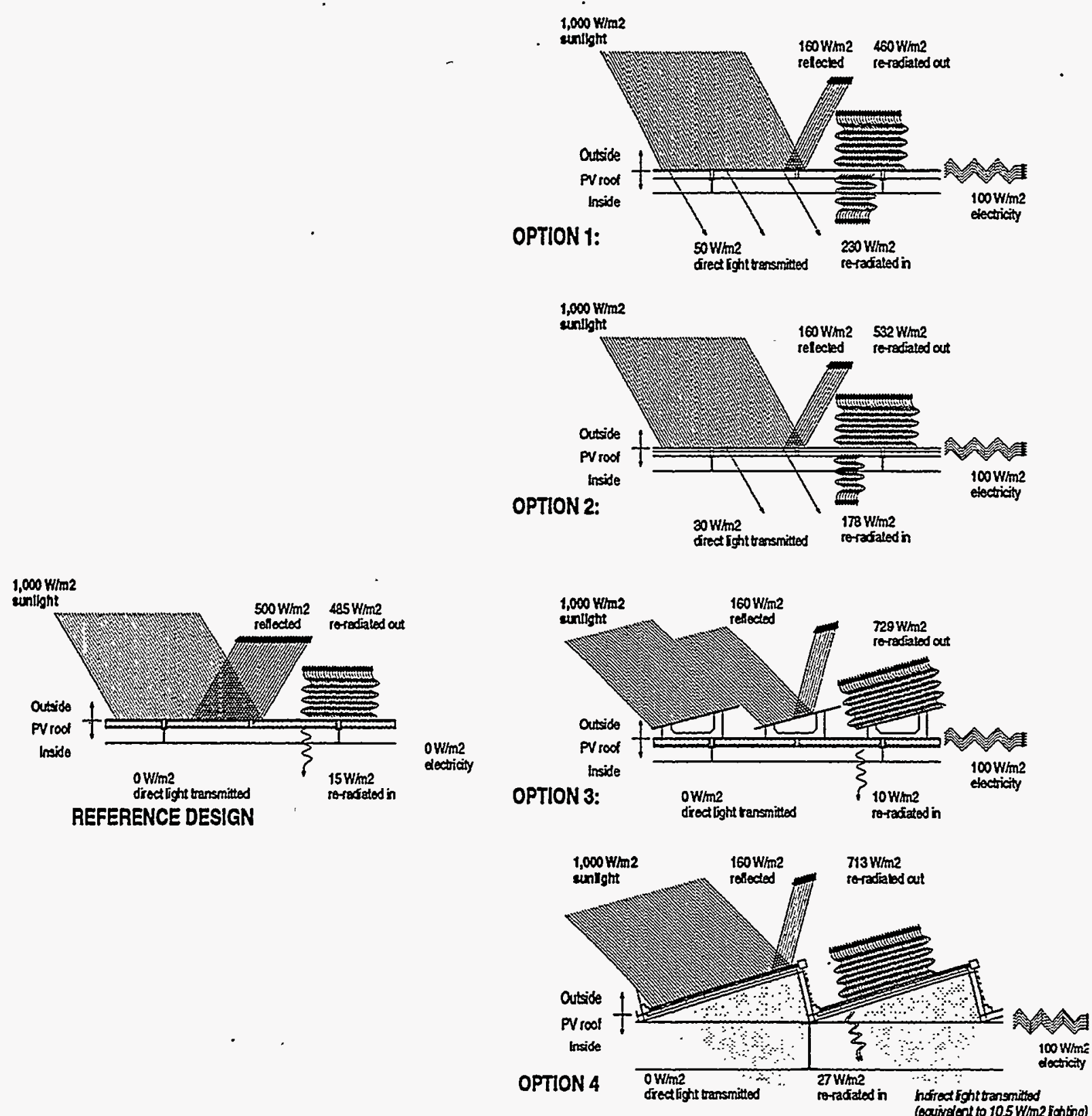

Note: these diagrams depict the energy balance of the roof systems under direct radiation (daylight) only. The energy balance analyses in the next section take into account the full range of daily and annual weather conditions.

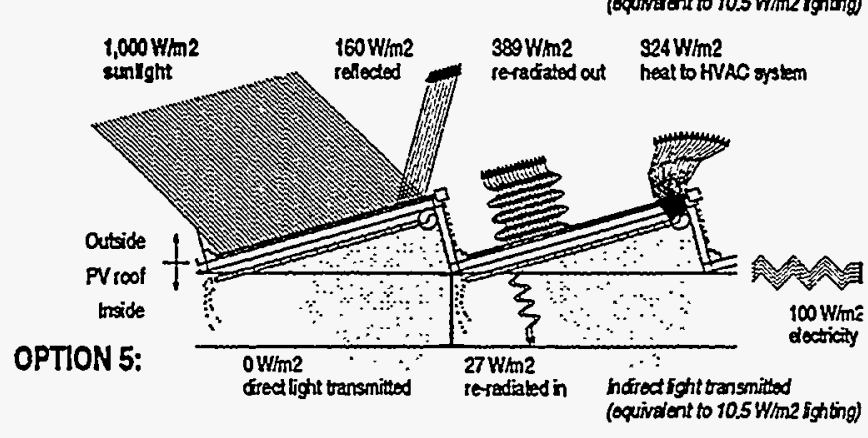




\section{ENERGY PERFORMANCE EVALUATIONS}

For each of the BIPV roof options, building load data was calculated from a building energy balance model developed by Mahadev Raman at Ove Arup \& Partners New York. We evaluated each roof option for its effect on the total building energy use in six different locations in the continental United States. These locations were chosen to represent a range of climate types as follows:

Oakland

New York (Long Island)

Phoenix

Miami

Chicago/Cincinnati
Temperate/coastal/warm

Temperate/coastal/cold

Temperate/arid

Subtropical

Temperate/continental

The effect of each roof system on typical building HVAC and lighting loads was calculated, taking into account:

- Gains from direct and indirect radiation

- Thermal transmittance in and out by conduction

- Offset electrical and thermal loads due to reduced artificial lighting.

The overall energy performance was compared to the reference roof design: flat, insulated dark colored with a U-value (metric) of 0.3 (R28.4 in english units).

The energy consumption of the test building for each roof option in each climate is shown in charts 1-7 on the following pages. The analyses show that Option 1 has poor energy performance in all climates, especially in climates with extremes of hot and cold, due to the poor insulating value of single glazing.

Option 2 (insulated glass roof) has better thermal energy performance than the conventional reference design in every climate except Phoenix, indicating that the daylighting and (to a lesser extent) the useful thermal gains through the glass more than offset it's lesser insulating value. Option 3 has very slightly better thermal performance due to the reduction in heat gain from the shading of the roof. Options 4 and 5 have significantly better energy performance than the reference design in every case. 


\section{OAKLAND: BUILDING LOADS FOR5 ROOFOPTIONS}

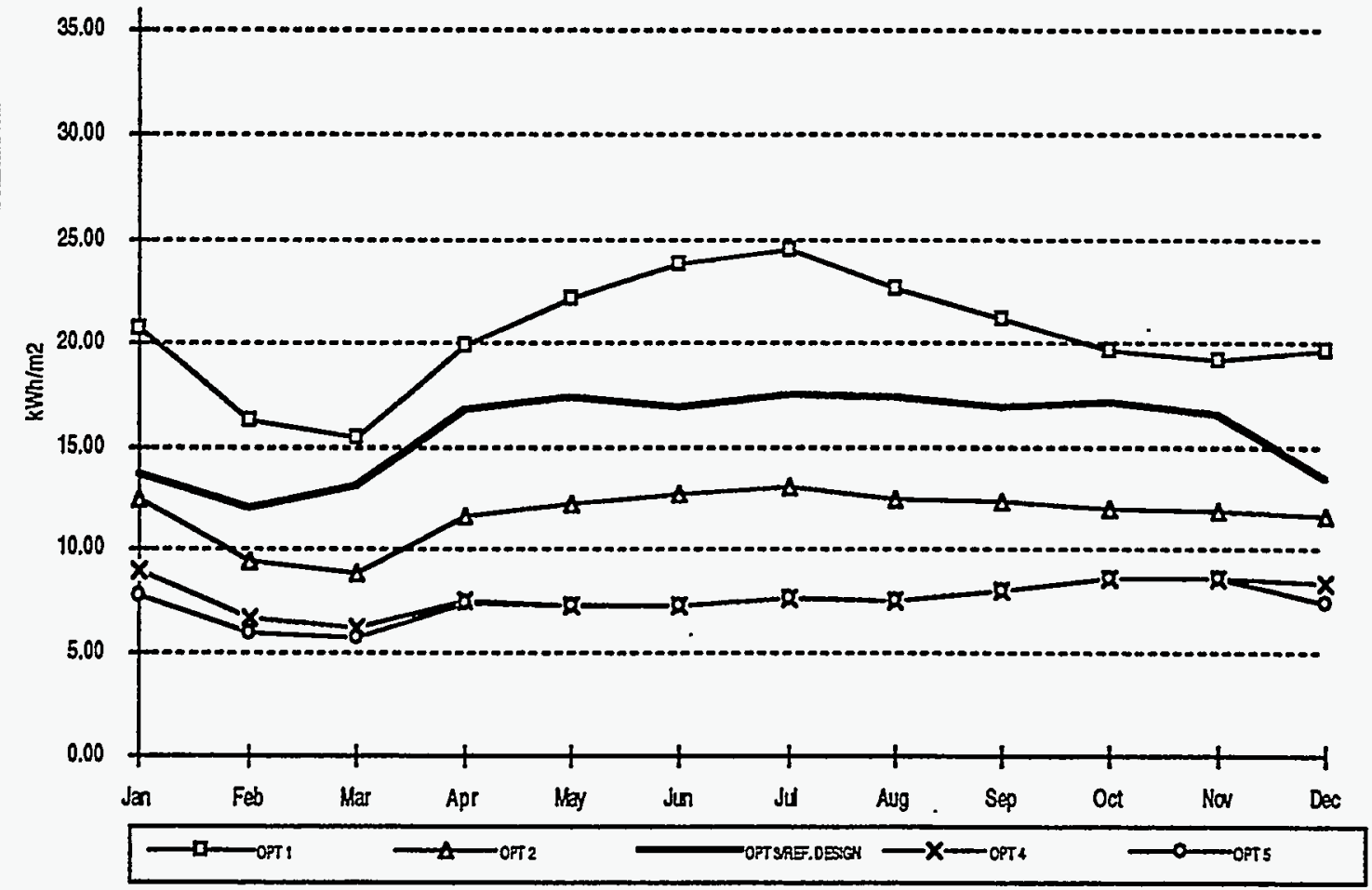

Chart 1. Energy performance for Oakland, California.

NEW YORK: BUILDING LOADS FOR 5 ROOF OPTIONS

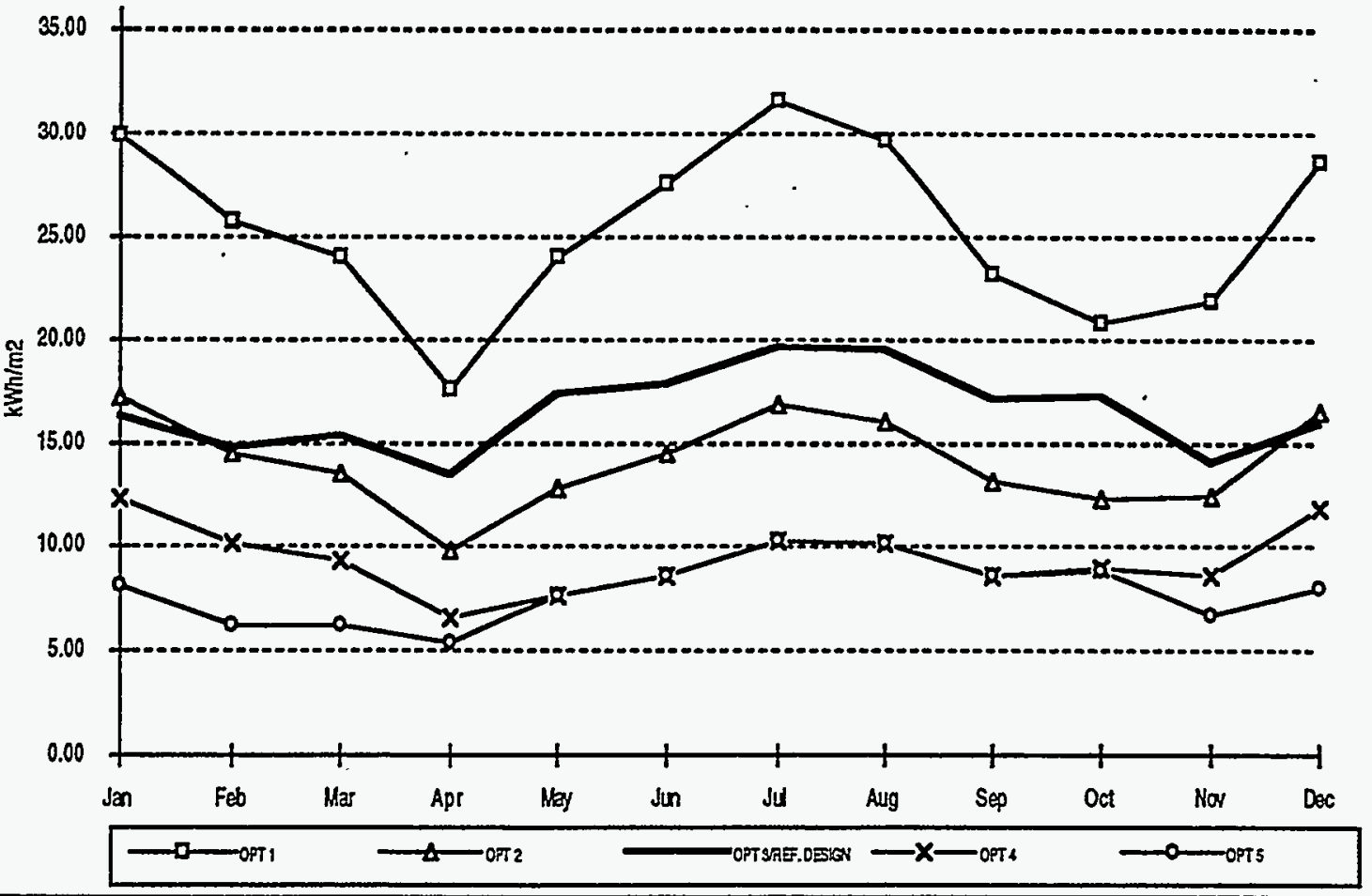

Chart 2. Energy performance for New York City, New York. 


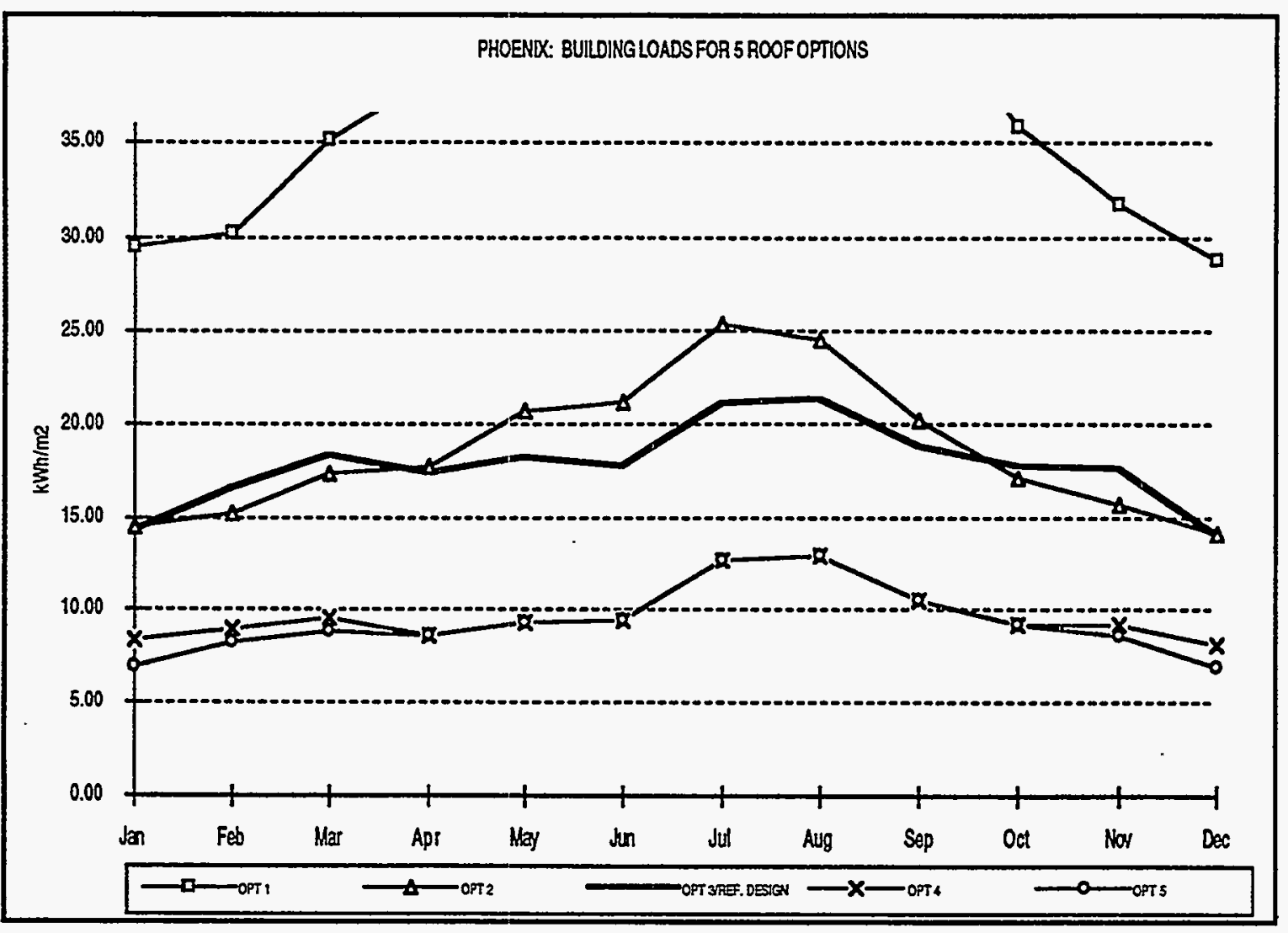

Chart 3. Energy performance for Phoenix, Arizona (extreme data for Option 1 not depicted on graph).

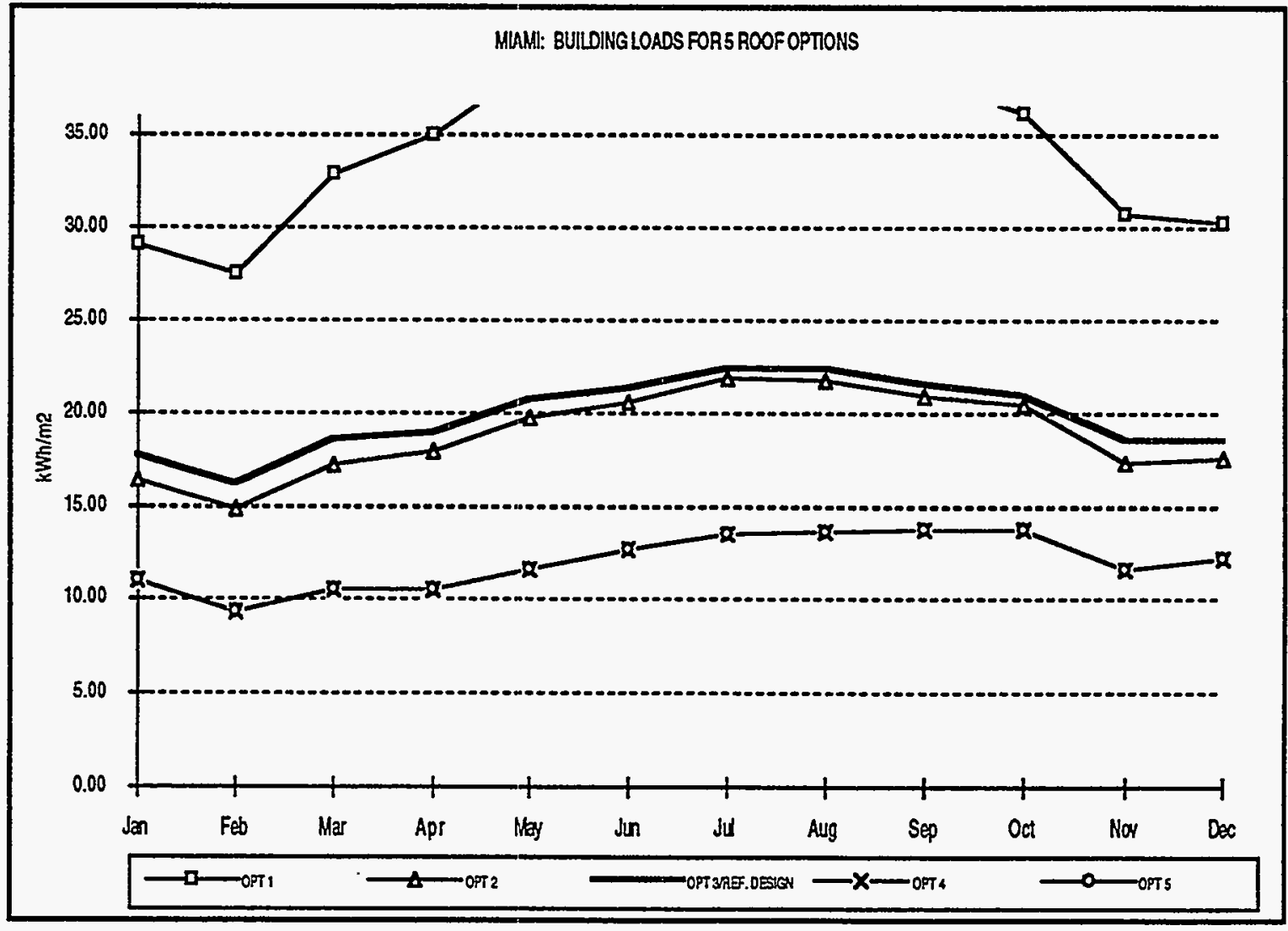

Chart 4. Energy performance for Miami, Florida (extreme data for Option 1 not depicted on graph). 


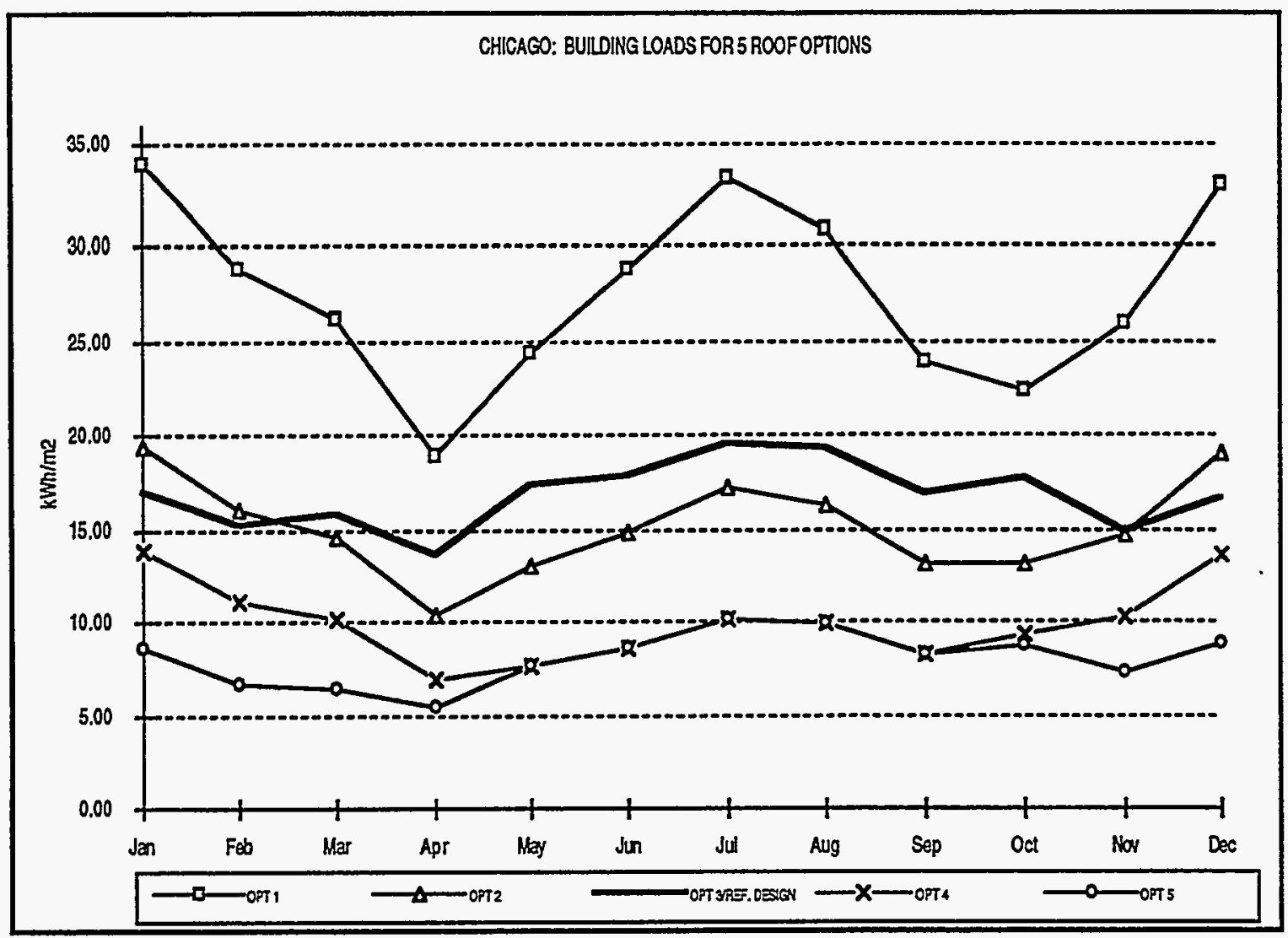

Chart 5. Energy performance for Chicago, Ilinois.

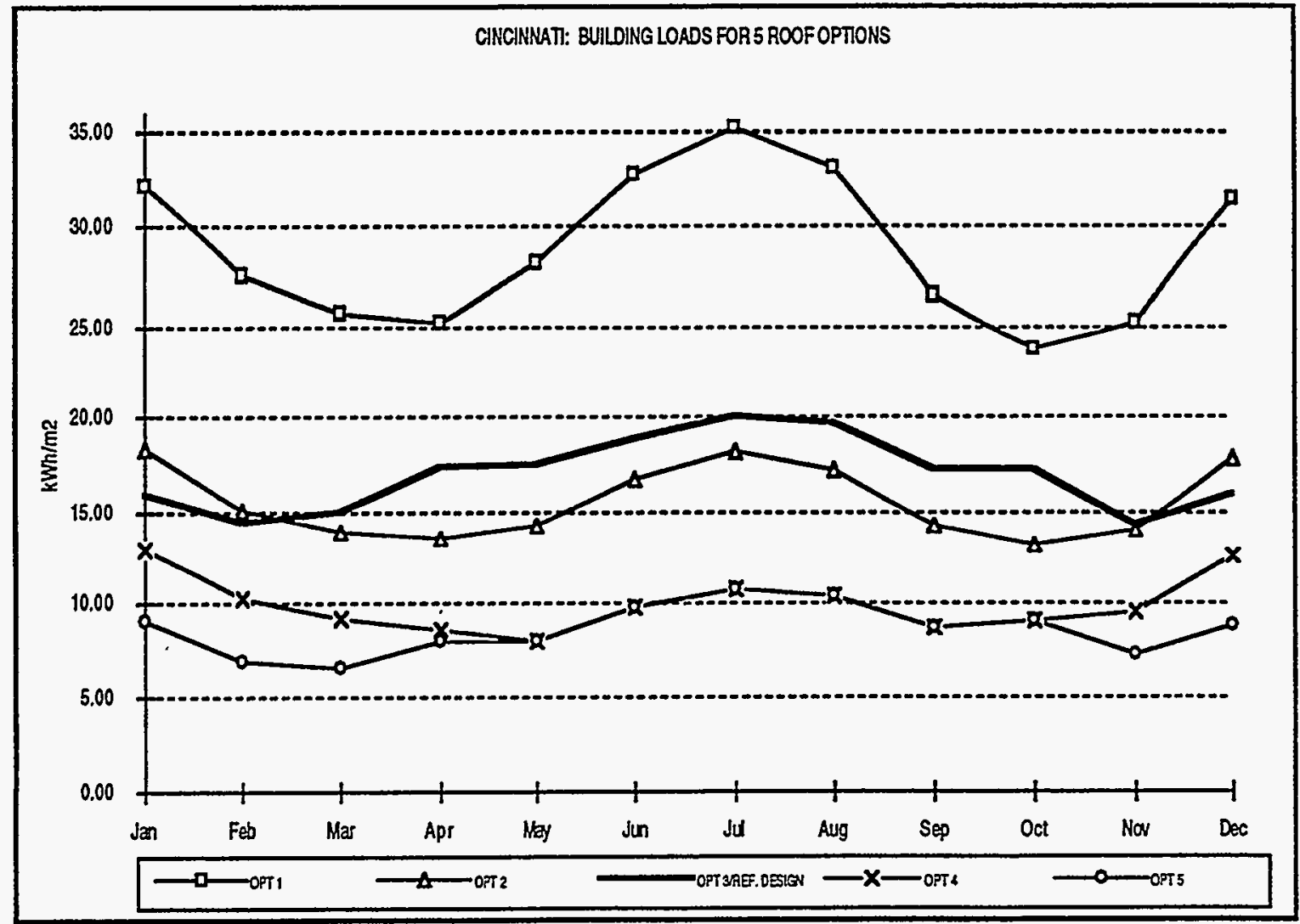

Chart 6. Energy performance for Cincinnati, Ohio. 


\section{PV PERFORMANCE CALCULATIONS}

In addition to the building load calculations, PV performance for each of the options was modeled using PV-F Chart software. PV output was calculated for each of the five BIPV options, in each of the six cities, for each of three PV technologies. The parameters are summarized as follows:

\section{PV Technology}

$\begin{array}{lll}\text { Type } & \text { Efficiency } & \text { Temperature coefficient } \\ \text { PV1 } & 13 \% & .0043\left(1 /{ }^{\circ} \mathrm{C}\right) \\ \text { PV2 } & 5.2 \% & .0027\left(1 /{ }^{\circ} \mathrm{C}\right) \\ \text { PV3 } & 10 \% & .0035\left(1 /{ }^{\circ} \mathrm{C}\right)\end{array}$

\section{Option-specific temperature parameters}

Different construction systems will cause the PVs to operate at different temperatures. All PVs lose efficiency at higher temperatures. The temperature coefficient above is the rate at which the module efficiency linearly decreases with temperature. Since the construction of each option affects the operating temperature of the modules, this factor further affects the total performance of each system.

$\begin{array}{cl}\text { Option } & \text { Cell operating temperature }\left({ }^{\circ} \mathrm{C} \text { above ambient) }\right. \\ 1 & +23.0^{\circ} \\ 2 & +26.6^{\circ} \\ 3 & +18.3^{\circ} \\ 4 & +35.7^{\circ} \\ 5 & +19.5^{\circ}\end{array}$

Other parameters were constant for all options. All options were evaluated at a $15^{\circ}$ altitude tilt. Various azimuth angles were evaluated (see Utility rate analysis.)

PV output was evaluated on an hourly basis for use in the utility rate calculations (see appendix D). 


\section{COST ANALYSIS}

This section estimates the cost premium of each roof option over the cost of a conventional flat non-PV roof. The conventional roof includes steel structure, metal deck, rigid insulation, and membrane roofing (see Fig. 22). Detailed breakdowns of the costs of each option are presented in subsequent tables. Basic assumptions concerning construction and PV technology are as follows:

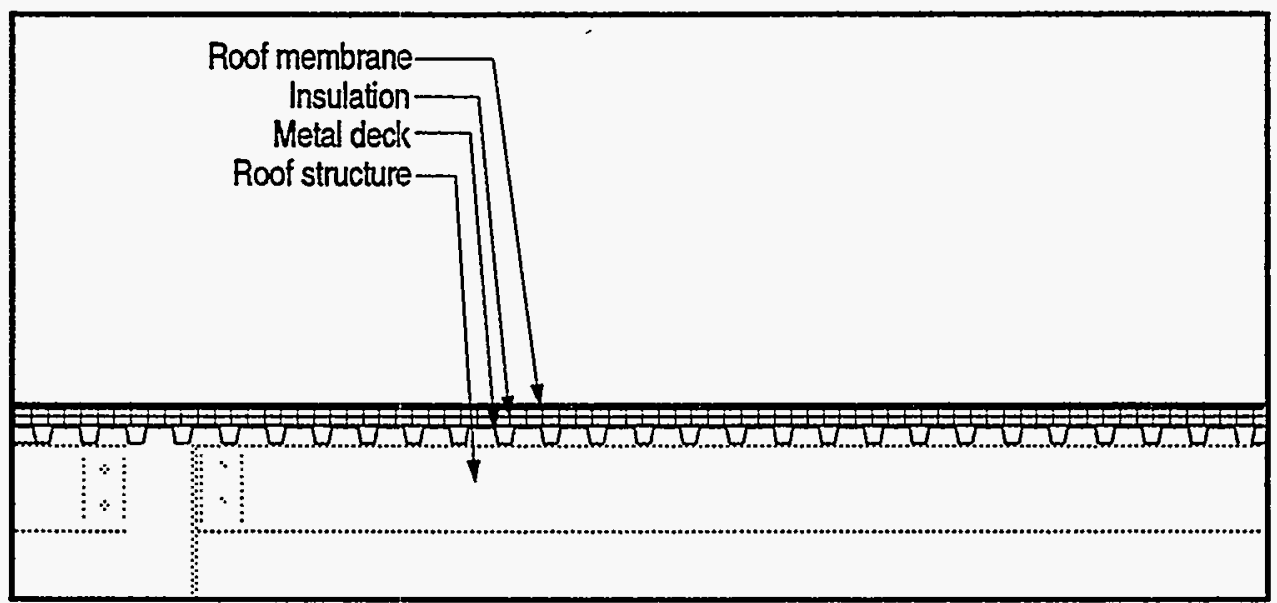

Fig 22. Section through typical bay, Reference Design

- Construction costs include any additional structure, insulation, roofing materials, glazing and sprinklers, beyond what is included in the reference roof.

- Costs of construction materials used in the standard roof that are replaced by a BIPV material are deducted from the cost of the BIPV system.

- PV system costs include modules, fabrication of insulating units (where applicable), aluminum framing to support the modules in a watertight seal (where applicable), wiring, and power conditioning.

- Three different PV technologies are evaluated, ranging from high-efficiency, high-cost/W to low-efficiency, low cost/W:

PV1: Crystalline Silicon

$140 \mathrm{~W} / \mathrm{m} 2 \$ 4.40 / \mathrm{W}$

Source: Arthur D Little report 6/94.

PV2: Amorphous Silicon $52 \mathrm{~W} / \mathrm{m} 2 \quad \$ 3.00 / \mathrm{W}$

Source: Advanced Photovoltaics Systems, Inc., 1994.

PV3: High-efficiency thin-film (CIS, CdTe) $\quad 108 \mathrm{~W} / \mathrm{m} 2 \quad \$ 2.00 / \mathrm{W}$

Source: Energy Photovoltaics, Inc. Projected price for 1996-97. 
PV1 and PV2 represent currently available technologies. PV3 cost/performance figures may be available in the near future using any number of technologies under development. PV3 projections are included because any significant construction project currently in planning stages may not be constructed for several years, at which point the new technologies, or improved versions of existing technologies, may be available.

Table 1 summarizes the construction costs of each option with each of the three PV technologies. (see Payback Analysis for a discussion of the implications of costs) For options 4 and 5, the costs and benefits are evaluated in three ways:

- As a daylight/thermal system only, including all construction costs associated with daylighting and/or thermal energy systems, but no PV-related costs. The payback analysis includes benefits (or costs) associated with daylighting and thermal performance only.

- As a PV system only. To isolate the costs and payback of the PV system, construction costs associated with daylighting and thermal systems are not included, as are any energy savings (or costs) produced by these systems. Only PV income is used.

- As a complete system, including all construction costs associated with daylighting and/or thermal energy systems. In the payback analysis, all benefits (or costs) associated with daylighting and thermal performance are included with PV income.

\begin{tabular}{|c|c|c|c|c|c|c|}
\hline \multirow[t]{2}{*}{ Costs:Fullsystem } & \multicolumn{2}{|r|}{ PV1 } & \multicolumn{2}{|r|}{ PV2 } & \multicolumn{2}{|c|}{ PV3 } \\
\hline & $\$ / m 2$ & $\$ / W$ & $\$ / m 2$ & $\$ / W$ & $\$ / m 2$ & $\$ / W$ \\
\hline OPTION 1 & $\$ 1,115.34$ & $\$ 7.97$ & $\$ 463.71$ & $\$ 8.97$ & $\$ 610.28$ & $\$ 5.67$ \\
\hline OPTION 1a & $\$ 863.30$ & $\$ 6.17$ & $\$ 211.68$ & $\$ 4.10$ & $\$ 358.25$ & $\$ 3.33$ \\
\hline OPTION 2 & $\$ 1,338.16$ & $\$ 9.56$ & $\$ 686.53$ & $\$ 13.29$ & $\$ 833.10$ & $\$ 7.74$ \\
\hline OPTION 2a & $\$ 897.97$ & $\$ 6.42$ & $\$ 246.34$ & $\$ 4.77$ & $\$ 392.91$ & $\$ 3.65$ \\
\hline OPTION 3 & $\$ 1,102.22$ & $\$ 7.88$ & $\$ 450.59$ & $\$ 8.72$ & $\$ 597.16$ & $\$ 5.55$ \\
\hline OPTION 4 & $\$ 1,149.86$ & $\$ 8.22$ & $\$ 498.24$ & $\$ 9.64$ & $\$ 644.80$ & $\$ 5.99$ \\
\hline OPTION 5, 5a & $\$ 1,424.67$ & $\$ 10.18$ & $\$ 773.05$ & $\$ 14.96$ & $\$ 919.61$ & $\$ 8.54$ \\
\hline \multicolumn{7}{|c|}{ Daylight+ThermalOnly } \\
\hline OPTION 4 & $\$ 136.77$ & NA & $\$ 136.77$ & NA & $\$ 136.77$ & NA \\
\hline OPTION 5, 5a & $\$ 235.80$ & NA & $\$ 235.80$ & NA & $\$ 235.80$ & NA \\
\hline \multicolumn{7}{|l|}{ PV systemonly } \\
\hline OPTION 4 & $\$ 1,013.09$ & $\$ 7.24$ & $\$ 361.46$ & $\$ 7.00$ & $\$ 508.03$ & $\$ 4.72$ \\
\hline OPTION 5, 5a & $\$ 1,188.87$ & $\$ 8.50$ & $\$ 537.24$ & $\$ 10.40$ & $\$ 683.81$ & $\$ 6.35$ \\
\hline
\end{tabular}

Table 1. A summary of the system costs (construction plus PV integration) for each of the five roof options, for each of the three different PV technologies. 


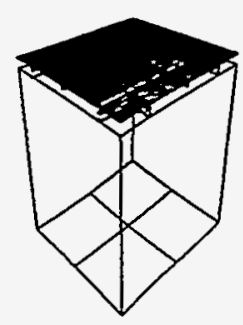

\section{OPTION 1 CONSTRUCTION COSTS}

\begin{tabular}{|c|c|c|c|c|c|c|}
\hline & $\mathrm{s} / \mathrm{m} 2$ & $\begin{array}{c}\text { PV1 } \\
\text { S/W }\end{array}$ & $\$ / m 2$ & $\begin{array}{c}\text { PV2 } \\
S N W\end{array}$ & $\$ / m 2$ & $\begin{array}{l}\text { PV3 } \\
\text { SNW }\end{array}$ \\
\hline $\begin{array}{l}1 \text { Additional roof structure } \\
2 \\
3 \text { PV system costs: }\end{array}$ & $\$ 0.00$ & 50.00 & $\$ 0.00$ & $\$ 0.00$ & $\$ 0.00$ & $\$ 0.00$ \\
\hline $\begin{array}{l}4 \text { PV modules } \\
5\end{array}$ & $\$ 615.72$ & $\$ 4.40$ & $\$ 155.01$ & $\$ 3.00$ & $\$ 215.29$ & $\$ 2.00$ \\
\hline 6 PV framing & $\$ 215.29$ & $\$ 1.54$ & $\$ 215.29$ & $\$ 4.17$ & $\$ 215.29$ & $\$ 2.00$ \\
\hline 7 PV wiring & $\$ 34.88$ & $\$ 0.25$ & $\$ 34.88$ & $\$ 0.68$ & $\$ 34.88$ & S0.32 \\
\hline 8 Other indirect & $\$ 55.97$ & SO. 40 & $\$ 20.67$ & S0.40 & $\$ 43.06$ & $\$ 0.40$ \\
\hline 9 Power Conditioning & $\$ 111.95$ & 50.80 & $\$ 41.33$ & So.80 & $\$ 86.11$ & $\$ 0.80$ \\
\hline $\begin{array}{l}10 \\
11 \text { Material credits: }\end{array}$ & $\$ 1,033.80$ & $\$ 7.39$ & $\$ 467.17$ & $\$ 9.04$ & $\$ 594.62$ & $\$ 5.52$ \\
\hline 12 Roofing & (\$21.53) & $(\$ 0.15)$ & $(\$ 21.53)$ & $(\$ 0.42)$ & $(\$ 21.53)$ & $(\$ 0.20)$ \\
\hline 13 Insulation & (\$20.88) & $(\$ 0.15)$ & $(\$ 20.88)$ & $(\$ 0.40)$ & $(\$ 20.88)$ & (\$0.19) \\
\hline 14 Metal Deck & $(\$ 21.53)$ & $(\$ 0.15)$ & $(\$ 21.53)$ & $(\$ 0.42)$ & $(\$ 21.53)$ & $(\$ 0.20)$ \\
\hline $\begin{array}{l}15 \\
16\end{array}$ & $(\$ 63.94)$ & $(\$ 0.46)$ & $(\$ 63.94)$ & $(\$ 1.24)$ & $(\$ 63.94)$ & (\$0.59) \\
\hline 17 Subtotal & $\$ 969.86$ & $\$ 6.93$ & $\$ 403.23$ & $\$ 7.80$ & $\$ 530.68$ & $\$ 4.93$ \\
\hline 18 Markup (15\%) & $\$ 145.48$ & $\$ 1.04$ & $\$ 60.48$ & $\$ 1.17$ & $\$ 79.60$ & $\$ 0.74$ \\
\hline 19 Total system & $\$ 1,115.34$ & $\$ 7.97$ & $\$ 463.71$ & $\$ 8.97$ & $\$ 610.28$ & $\$ 5.67$ \\
\hline
\end{tabular}

Table 2. Detailed breakdown of Option I cost calculations.

\section{TABLE NOTES:}

6. PV framing. Aluminum extrusion skylight system. Source: DeaMor, Inc. 1994

7. PV wiring. $\$ 37.67 / \mathrm{m} 2(\$ 3.50 / \mathrm{st}) \mathrm{PV}$ area allowance. Could be significantly less: assuming modules are connected in series of six (a-Si modules @ $40 \mathrm{~V}$ ea for a $240 \mathrm{~V}$ system) with snap together connectors in the series string, conduit is needed only for wiring between strings and for home runs to the inverter. In the test building, this should require approx. $1 \mathrm{~m}$ conduit $/ 10 \mathrm{~m} 2 \mathrm{PV}$ area, or $\$ 10.00 / \mathrm{m} 2 @ \$ 10 / \mathrm{m}$ wiring cost allowance.

8. Other indirect. Engineering, permits, shipping, insurance, project management. Source: AD Little 6/94.

9. Power conditioning. Source: various industry reports. This figure should be conservative for a system of over 100kW. APS-Fairfield data suggest a cost of under $\$ 0.60 / \mathrm{W}$ for a system this size.

12. Roofing credit. 55 mil EPDM fully adhered membrane. Source: Deocon Construction Inc., 1994.

13. Insulation. Phenolic foam board 2 layers $\times 1.75^{\prime \prime},(\mathrm{R} 28.4)$ Source: RS Means Construction Cost Data 1993.

14. Metal deck. Source: Devcon Construction Inc., 1994. 


\section{SECTION}

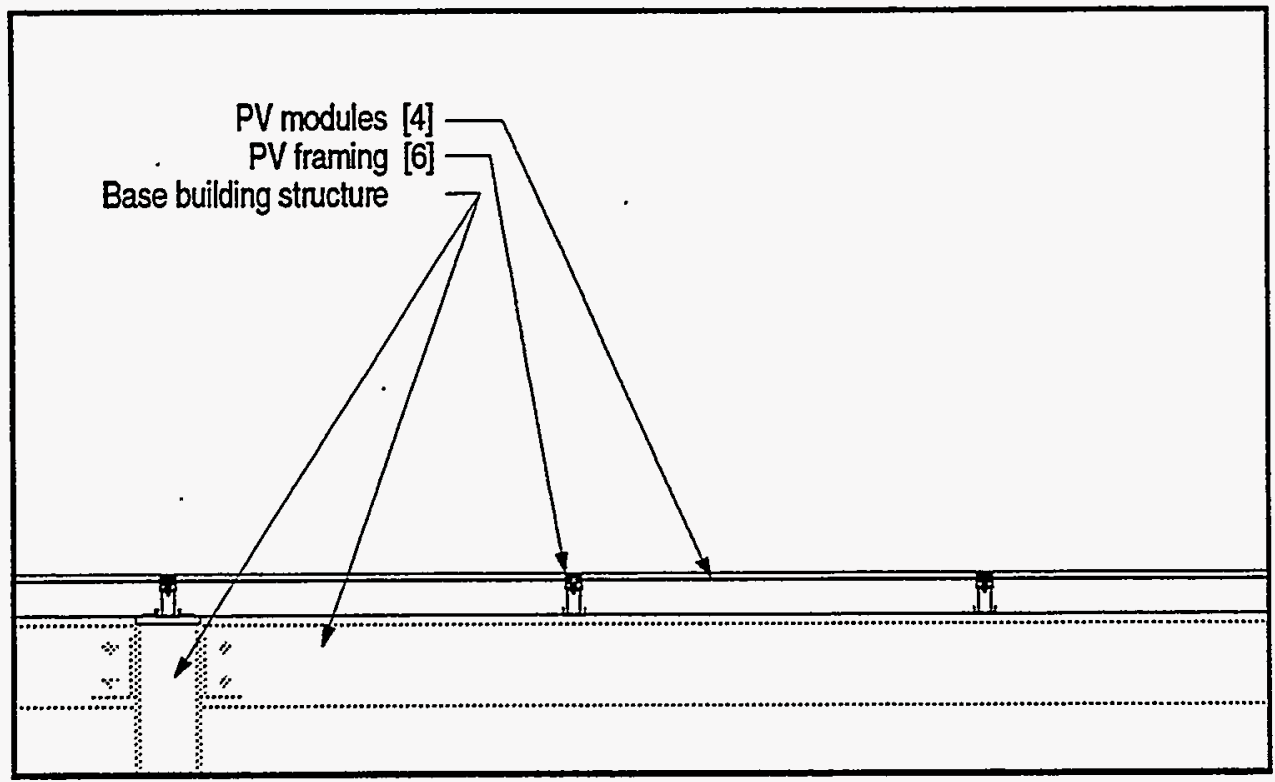

Fig 23. Section through typical bay, Option I 


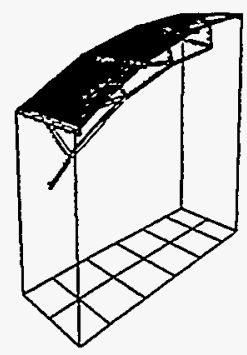

\section{OPTION 1A CONSTRUCTION COSTS}

\begin{tabular}{|c|c|c|c|c|c|c|}
\hline & $\$ / m 2$ & $\begin{array}{r}\text { PV1 } \\
\$ / W\end{array}$ & $\mathrm{~s} / \mathrm{m} 2$ & $\begin{array}{l}\text { PV2 } \\
\text { S/W }\end{array}$ & s/m2 & $\begin{array}{l}\text { PV3 } \\
\text { S/W }\end{array}$ \\
\hline $\begin{array}{l}1 \text { Additional roof structure } \\
2 \\
3 \text { pV sustem costs. }\end{array}$ & $\$ 0.00$ & $\$ 0.00$ & $\$ 0.00$ & $\$ 0.00$ & $\$ 0.00$ & $\$ 0.00$ \\
\hline 4 PV modules & $\$ 615.72$ & $\$ 4.40$ & $\$ 155.01$ & $\$ 3.00$ & $\$ 215.29$ & $\$ 2.00$ \\
\hline 6 PV framing & $\$ 0.00$ & $\$ 0.00$ & $\$ 0.00$ & $\$ 0.00$ & $\$ 0.00$ & $\$ 0.00$ \\
\hline 7 PV wiring & $\$ 34.88$ & $\$ 0.25$ & $\$ 34.88$ & $\$ 0.68$ & $\$ 34.88$ & \$0.32 \\
\hline 8 Other indirect & $\$ 55.97$ & $\$ 0.40$ & $\$ 20.67$ & $\$ 0.40$ & $\$ 43.06$ & $\$ 0.40$ \\
\hline 9 Power Conditioning & $\$ 111.95$ & $\$ 0.80$ & $\$ 41.33$ & $\$ 0.80$ & $\$ 86.11$ & $\$ 0.80$ \\
\hline 10 & $\$ 818.51$ & $\$ 5.85$ & $\$ 251.88$ & $\$ 4.88$ & $\$ 379.33$ & $\$ 3.52$ \\
\hline \multicolumn{7}{|l|}{11 Material credits: } \\
\hline 12 Roofing & $\$ 0.00$ & $\$ 0.00$ & $\$ 0.00$ & $\$ 0.00$ & $\$ 0.00$ & $\$ 0.00$ \\
\hline 13 Insulation & $\$ 0.00$ & $\$ 0.00$ & $\$ 0.00$ & $\$ 0.00$ & $\$ 0.00$ & $\$ 0.00$ \\
\hline 14 Glazing & (\$67.81) & $(\$ 0.48)$ & $(\$ 67.81)$ & $(\$ 1.31)$ & (\$567.81) & $(\$ 0.63)$ \\
\hline 15 & (\$67.81) & $(\$ 0.48)$ & $(\$ 67.81)$ & $(\$ 1.31)$ & $(\$ 67.81)$ & $(\$ 0.63)$ \\
\hline \multicolumn{7}{|l|}{16} \\
\hline 17 Subtotal & $\$ 750.70$ & $\$ 5.36$ & $\$ 184.07$ & $\$ 3.56$ & $\$ 311.52$ & $\$ 2.89$ \\
\hline 18 Markup (15\%) & $\$ 112.60$ & $\$ 0.80$ & $\$ 27.61$ & $\$ 0.53$ & $\$ 46.73$ & $\$ 0.43$ \\
\hline 19 Totalsystem & $\$ 863.30$ & $\$ 6.17$ & $\$ 211.68$ & $\$ 4.10$ & $\$ 358.25$ & $\$ 3.33$ \\
\hline
\end{tabular}

Table 3. Detailed breakdown of Option 1 a cost calculations.

\section{TABLE NOTES:}

Option $1 \mathrm{~A}$ and $2 \mathrm{~A}$ are referenced to an atrium roof design, including skylight framing, glazing and exposed siructure.

7. PV wiring. $\$ 37.67 / \mathrm{m} 2(\$ 3.50 / \mathrm{sf}) \mathrm{PV}$ area allowance. Could be significantly less: assuming modules are connected in series of six (a-Si modules @ $40 \mathrm{~V}$ ea for a $240 \mathrm{~V}$ system) with snap together connectors in the series string, conduit is needed only for wiring between strings and for home runs to the inverter. In the test building, this should require approx. $1 \mathrm{~m}$ conduit $/ 10 \mathrm{~m} 2 \mathrm{PV}$ area, or $\$ 10.00 / \mathrm{m} 2 @ \$ 10 / \mathrm{m}$ wiring cost allowance.

8. Other indirect. Engineering, permits, shipping, insurance, project management. Source: AD Little 6/94.

9. Power conditioning. Source: various industry reports. This figure should be conservative for a system of over $100 \mathrm{~kW}$. APS-Fairfield data suggest a cost of under $\$ 0.60 / \mathrm{W}$ for a system this size.

12,13. Material credits. Since this option is identical to option 1 except that it is being compared to atrium roof construction, credits for the flat roof components are not used.

14. Glazing. Credit for $1 / 4$ " laminated, tinted glass. 


\section{SECTION}

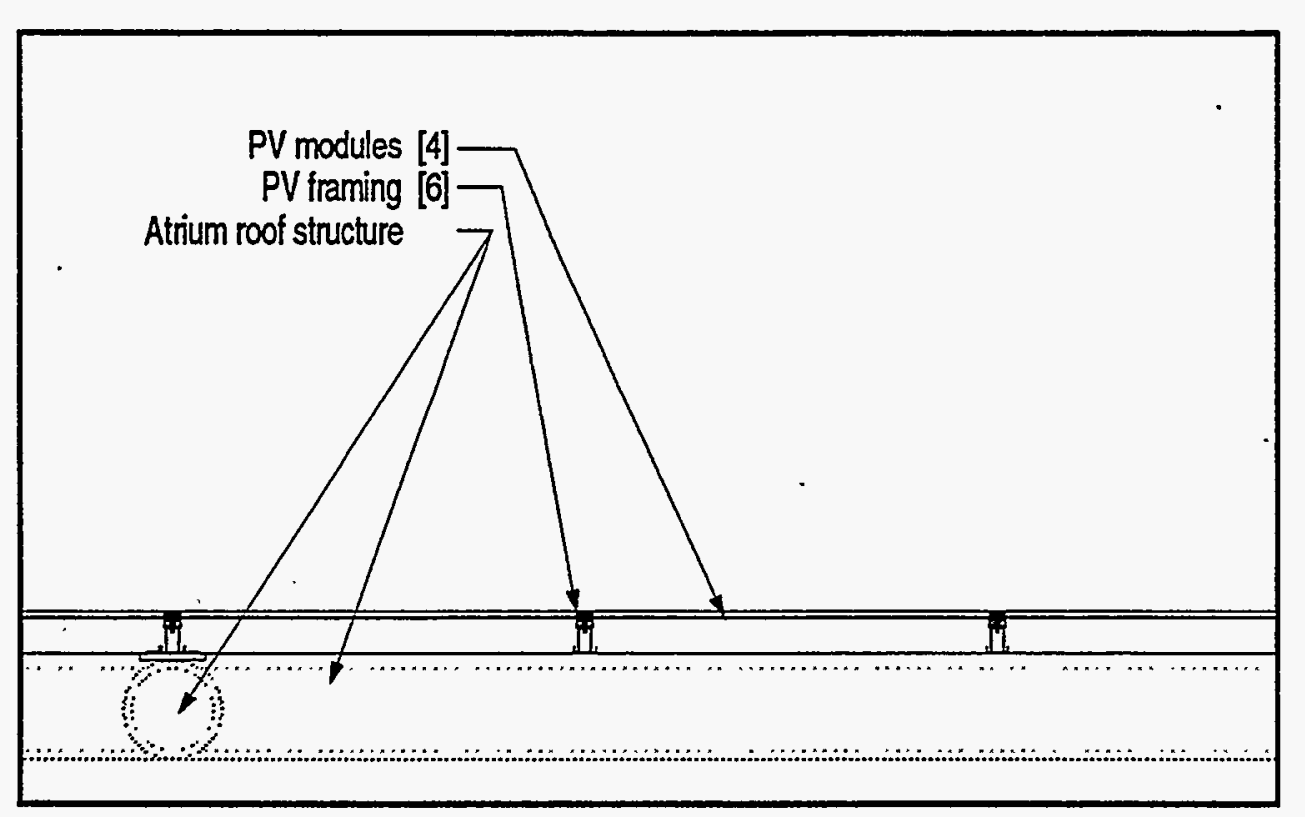

Fig 24. Section through typical bay, Option $1 a$ 


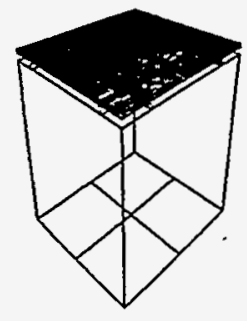

\section{OPTION 2 CONSTRUCTION COSTS}

\begin{tabular}{|c|c|c|c|c|c|c|}
\hline & $\$ / m 2$ & $\begin{array}{l}\text { PVI } \\
\text { S/W }\end{array}$ & $\$ / m 2$ & $\begin{array}{r}\text { PV2 } \\
\$ N W\end{array}$ & $\mathrm{~S} / \mathrm{m} 2$ & $\begin{array}{l}\text { PV3 } \\
\text { S/W }\end{array}$ \\
\hline $\begin{array}{l}1 \text { Additional roof structure } \\
2 \\
3 \text { PV system costs: }\end{array}$ & $\$ 0.00$ & $\$ 0.00$ & $\$ 0.00$ & $\$ 0.00$ & $\$ 0.00$ & $\$ 0.00$ \\
\hline 4 PV modules & $\$ 615.72$ & $\$ 4.40$ & $\$ 155.01$ & $\$ 3.00$ & $\$ 215.29$ & $\$ 2.00$ \\
\hline 5 IG unil fabrication & $\$ 193.76$ & $\$ 1.38$ & $\$ 193.76$ & $\$ 3.75$ & $\$ 193.76$ & $\$ 1.80$ \\
\hline 6 PV framing & $\$ 215.29$ & $\$ 1.54$ & $\$ 215.29$ & $\$ 4.17$ & $\$ 215.29$ & $\$ 2.00$ \\
\hline 7 PV wiring & $\$ 34.88$ & $\$ 0.25$ & $\$ 34.88$ & \$0.68 & $\$ 34.88$ & S0.32 \\
\hline 8 Other indirect & $\$ 55.97$ & $\$ 0.40$ & $\$ 20.67$ & $\$ 0.40$ & $\$ 43.06$ & \$0.40 \\
\hline 9 Power Conditioning & $\$ 111.95$ & $\$ 0.80$ & $\$ 41.33$ & $\$ 0.80$ & $\$ 86.11$ & 50.80 \\
\hline $\begin{array}{l}10 \\
11 \text { Material credits: }\end{array}$ & $\$ 1,227.56$ & $\$ 8.77$ & $\$ 660.93$ & $\$ 12.79$ & $\$ 788.37$ & $\$ 7.32$ \\
\hline 12 Roofing & $(\$ 21.53)$ & $(\$ 0.15)$ & (\$21.53) & $(\$ 0.42)$ & $(\$ 21.53)$ & $(\$ 0.20)$ \\
\hline 13 Insulation & (\$20.88) & $(\$ 0.15)$ & (\$20.88) & $(\$ 0.40)$ & $(\$ 20.88)$ & (\$0.19) \\
\hline 14 Metal Deck & $(\$ 21.53)$ & $(\$ 0.15)$ & $(\$ 21.53)$ & $(\$ 0.42)$ & $(\$ 21.53)$ & $(\$ 0.20)$ \\
\hline 15 & $(\$ 63.94)$ & $(\$ 0.46)$ & (\$63.94) & $(\$ 1.24)$ & $(\$ 63.94)$ & $(\$ 0.59)$ \\
\hline 16 & & & & & & \\
\hline 17 Subtotal & $\$ 1,163,62$ & $\$ 8.32$ & $\$ 596.99$ & $\$ 11.55$ & $\$ 724.43$ & $\$ 6.73$ \\
\hline 18 Markup (15\%) & $\$ 174.54$ & $\$ 1.25$ & $\$ 89.55$ & $\$ 1.73$ & $\$ 108.67$ & $\$ 1.01$ \\
\hline 19 Total & $\$ 1,338.16$ & $\$ 9.56$ & $\$ 686.53$ & $\$ 13.29$ & $\$ 833.10$ & $\$ 7.74$ \\
\hline
\end{tabular}

Table 4. Detailed breakdown of Option 2 cost calculations.

\section{TABLE NOTES:}

5. IG unit fabrication. $\$ 18.00 / \mathrm{sf}$ : Assumes $\$ 21.50 / \mathrm{sf}$ for insulating glazing unit with $1 / 4^{\prime \prime}$ float and $1 / 4^{\prime \prime}$ laminate glass less $\$ 3.50 /$ sf for replacing $1 / 4^{\prime \prime}$ float glass with PV.

6. PV framing. Aluminum extrusion skylight system. Source: DeaMor, Inc. 1994

7. PV wiring. $\$ 37.67 / \mathrm{m} 2$ ( $\$ 3.50 / \mathrm{sf})$ PV area allowance. Could be significantly less: assuming modules are connected in series of 6 (a-Si modules@ 40V ea for a $240 \mathrm{~V}$ system) with snap together connectors in the series string, conduit is needed only for wiring between strings and for home runs to the inverter. In the test building, this should require approx. $1 \mathrm{~m}$ conduit $/ 10 \mathrm{~m} 2 \mathrm{PV}$ area, or $\$ 10.00 / \mathrm{m} 2 @ \$ 10 / \mathrm{m}$ wiring cost allowance.

8. Other indirect. Engineering, permits, shipping, insurance, project management. Source: $A D$ Little 6/94.

9. Power conditioning. This figure should be conservative for a system of over $100 \mathrm{~kW}$. APS-Fairfield data suggest a cost of under $\$ 0.60 / \mathrm{W}$ for a system this size.

12. Roofing credit. 55 mil EPDM fully adhered membrane. Source: Devcon Construction Inc., 1994.

13. Insulation. Phenolic foam board 2 layers $\times 1.75^{\prime \prime},(\mathrm{R} 28.4)$ Source: RS Means Construction Cost Data 1993.

14. Metal deck. Source: Deocon Construction Inc, 1994. 
SECTION

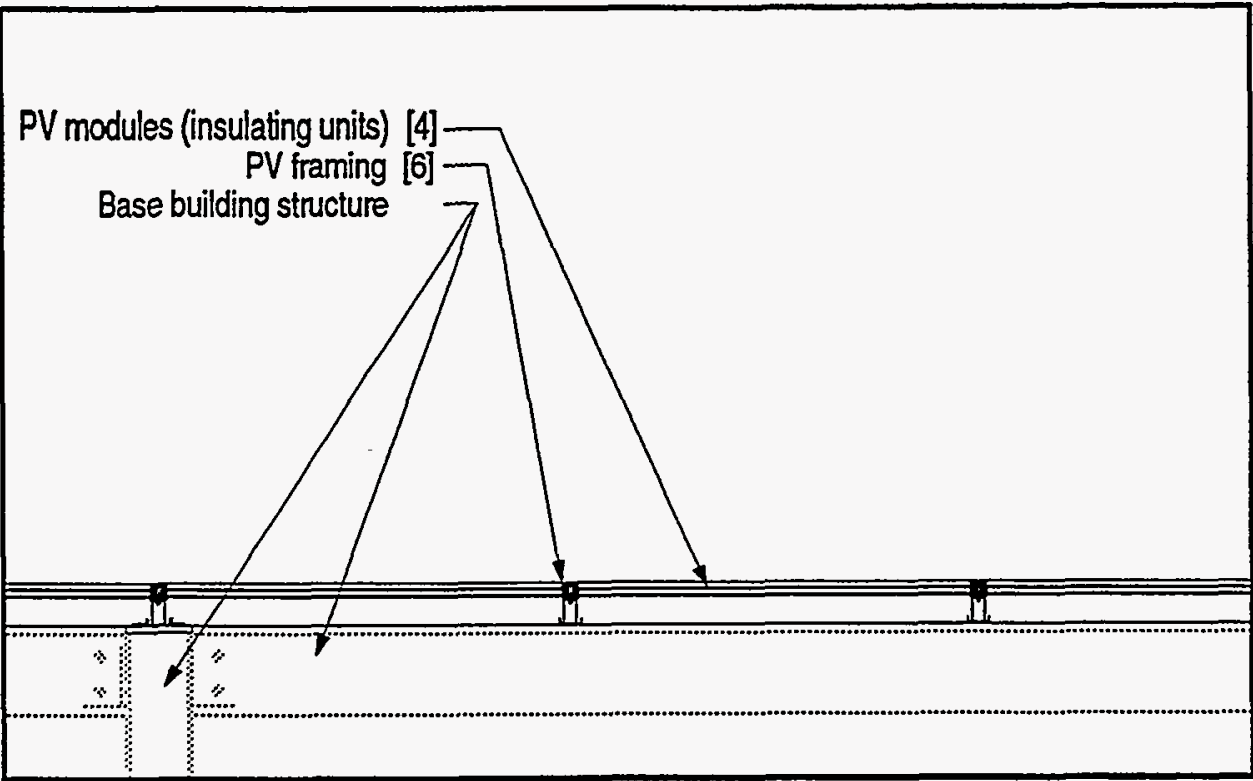

Fig 25. Section through typical bay, Option 2 


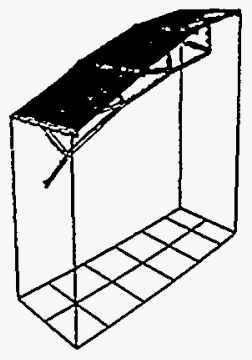

\section{OPTION 2A CONSTRUCTION COSTS}

\begin{tabular}{|c|c|c|c|c|c|c|}
\hline & $\mathrm{s} / \mathrm{m} 2$ & $\begin{array}{c}\text { PV1 } \\
\text { S/W }\end{array}$ & $\mathrm{s} / \mathrm{m} 2$ & $\begin{array}{c}\text { PV2 } \\
S / W\end{array}$ & $\mathrm{~s} / \mathrm{m} 2$ & $\begin{array}{l}\text { PV3 } \\
\text { SN }\end{array}$ \\
\hline $\begin{array}{l}1 \text { Additional roof structure } \\
2 \\
3 \text { PV system costs: }\end{array}$ & $\$ 0.00$ & $\$ 0.00$ & $\$ 0.00$ & $\$ 0.00$ & $\$ 0.00$ & $\$ 0.00$ \\
\hline 4 PV modules & $\$ 615.72$ & $\$ 4.40$ & $\$ 155.01$ & $\$ 3.00$ & $\$ 215.29$ & $\$ 2.00$ \\
\hline 5 Lg. area encaps. (IG units) & $\$ 193.76$ & $\$ 1.38$ & $\$ 193.76$ & $\$ 3.75$ & $\$ 193.76$ & $\$ 1.80$ \\
\hline 6 PV framing & $\$ 0.00$ & $\$ 0.00$ & $\$ 0.00$ & $\$ 0.00$ & $\$ 0.00$ & $\$ 0.00$ \\
\hline 7 PV wiring & $\$ 34.88$ & $\$ 0.25$ & $\$ 34.88$ & $\$ 0.68$ & $\$ 34.88$ & $\$ 0.32$ \\
\hline 8 Other indirect & $\$ 55.97$ & $\$ 0.40$ & $\$ 20.67$ & $\$ 0.40$ & $\$ 43.06$ & $\$ 0.40$ \\
\hline 9 Power Conditioning & $\$ 111.95$ & $\$ 0.80$ & $\$ 41.33$ & $\$ 0.80$ & $\$ 86.11$ & $\$ 0.80$ \\
\hline 10 & $\$ 1,012.27$ & $\$ 7.23$ & $\$ 445.64$ & $\$ 8.63$ & $\$ 573.09$ & $\$ 5.32$ \\
\hline \multicolumn{7}{|l|}{11 Material credits: } \\
\hline 12 Roofing & $\$ 0.00$ & $\$ 0.00$ & S0.00 & $\$ 0.00$ & So.00 & $\$ 0.00$ \\
\hline 13 Insulation & 50.00 & $\$ 0.00$ & $\$ 0.00$ & $\$ 0.00$ & $\$ 0.00$ & $\$ 0.00$ \\
\hline 14 Glazing & $(\$ 231.43)$ & $(\$ 1.65)$ & $(\$ 231.43)$ & $(\$ 4.48)$ & $(\$ 231.43)$ & $(\$ 2.15)$ \\
\hline 15 & $(\$ 231.43)$ & $(\$ 1.65)$ & (\$231.43) & $(\$ 4.48)$ & (\$231.43) & $(\$ 2.15)$ \\
\hline \multicolumn{7}{|l|}{16} \\
\hline 17 Subtotal & $\$ 780.84$ & $\$ 5.58$ & $\$ 214.21$ & $\$ 4.15$ & $\$ 341.66$ & $\$ 3.17$ \\
\hline 18 Markup (15\%) & $\$ 117.13$ & $\$ 0.84$ & $\$ 32.13$ & $\$ 0.62$ & $\$ 51.25$ & SO.48 \\
\hline 19 Total & $\$ 897.97$ & $\$ 6.42$ & $\$ 246.34$ & $\$ 4.77$ & $\$ 392.91$ & $\$ 3.65$ \\
\hline
\end{tabular}

Table 5. Detailed breakdown of Option $2 a$ cost calculations.

\section{TABLE NOTES:}

Option $1 \mathrm{~A}$ and $\mathrm{AA}$ are referenced to an atrium roof design, including skylight framing, glazing and exposed structure.

7. PV wiring. $\$ 37.67 / \mathrm{m} 2$ ( $\$ 3.50 / \mathrm{sf})$ PV area allowance. Could be significantly less: assuming modules are connected in series of 6 (a-Si modules @ $40 \mathrm{~V}$ ea for a $240 \mathrm{~V}$ system) with snap together connectors in the series string, conduit is needed only for wiring between strings and for home runs to the inverter. In the test building, this should require approx. $1 \mathrm{~m}$ conduit $/ 10 \mathrm{~m} 2 \mathrm{PV}$ area, or $\$ 10.00 / \mathrm{m} 2 @ \$ 10 / \mathrm{m}$ wiring cost allowance.

8. Other indirect. Engineering, permits, shipping, insurance, project management. Source: AD Little 6/94.

9. Power conditioning. This figure should be conservative for a system of over $100 \mathrm{~kW}$. APS-Fairfield data suggest a cost of under $\$ 0.60 / \mathrm{W}$ for a system this size.

11. Material credits. Since this option is identical to option 2 except that it is being compared to atrium roof construction, credits for the flat roof components are not used.

14. Glazing. Credit for $1^{\prime \prime}$ double glazing, $1 / 4^{\prime \prime}$ float, $1 / 4^{\prime \prime}$ wire glass. 


\section{SECTION}

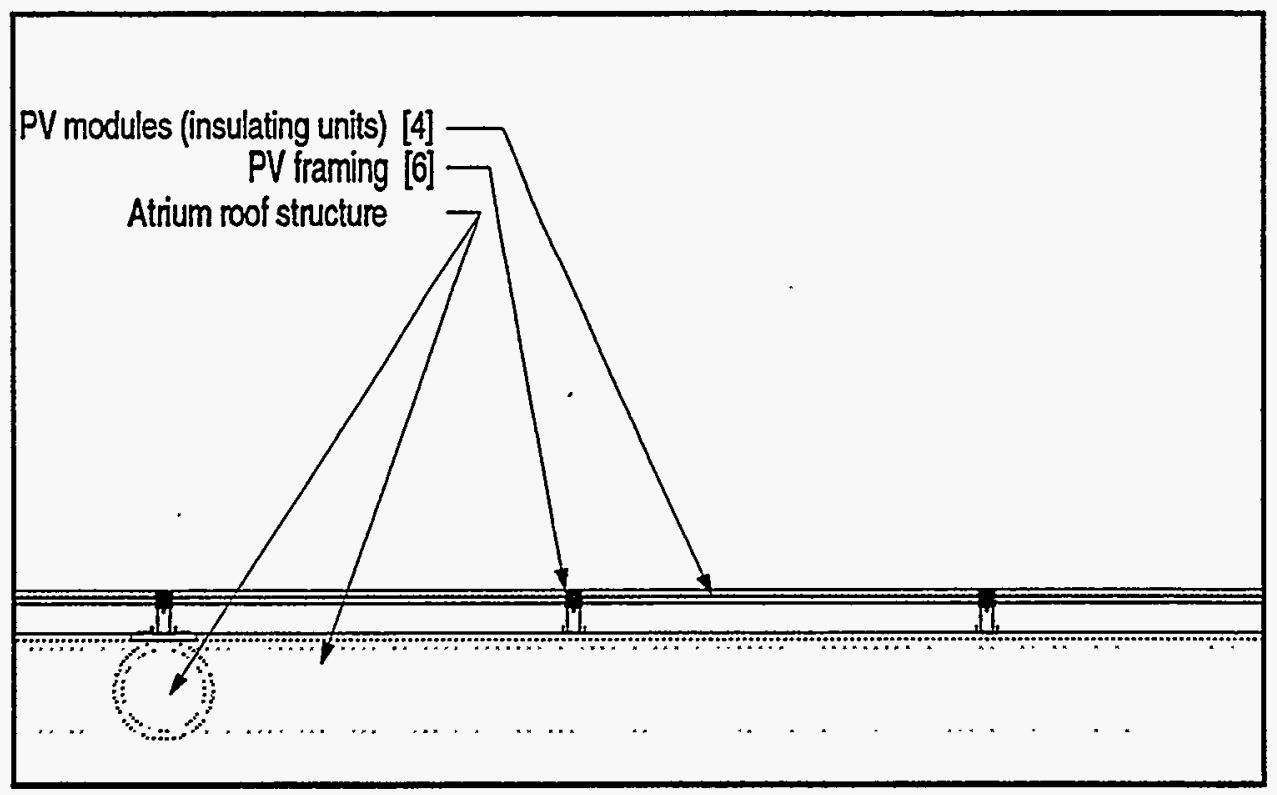

Fig 26. Section through typical bay, Option $2 a$ 


\section{OPTION 3 CONSTRUCTION COSTS}

\begin{tabular}{|c|c|c|c|c|c|c|}
\hline & $\$ / m 2$ & $\begin{array}{l}\text { PV1 } \\
\text { SNW }\end{array}$ & $\mathrm{s} / \mathrm{m}^{2}$ & $\begin{array}{c}\text { PV2 } \\
S / W\end{array}$ & $\mathrm{~s} / \mathrm{m} 2$ & $\begin{array}{l}\text { PV3 } \\
\text { S/W }\end{array}$ \\
\hline $\begin{array}{l}1 \text { Ballasted roof structure } \\
2 \\
3 \text { PV system costs: }\end{array}$ & $\$ 139.94$ & $\$ 1.00$ & $\$ 139.94$ & $\$ 2.71$ & $\$ 139.94$ & $\$ 1.30$ \\
\hline $\begin{array}{l}4 \text { PV modules } \\
5\end{array}$ & $\$ 615.72$ & $\$ 4.40$ & $\$ 155.01$ & $\$ 3.00$ & $\$ 215.29$ & $\$ 2.00$ \\
\hline 6 PV wiring & $\$ 34.88$ & $\$ 0.25$ & $\$ 34.88$ & $\$ 0.68$ & $\$ 34.88$ & \$0.32 \\
\hline 7 Other indirect & $\$ 55.97$ & $\$ 0.40$ & $\$ 20.67$ & $\$ 0.40$ & $\$ 43.06$ & S0.40 \\
\hline 8 Power Conditioning & $\$ 111.95$ & S0.80 & $\$ 41.33$ & $\$ 0.80$ & $\$ 86.11$ & $\$ 0.80$ \\
\hline 9 & $\$ 958.45$ & $\$ 6.85$ & $\$ 391.82$ & $\$ 7.58$ & $\$ 519.27$ & $\$ 4.82$ \\
\hline \multicolumn{7}{|l|}{10} \\
\hline \multicolumn{7}{|l|}{11 Material credits: } \\
\hline \multicolumn{7}{|l|}{12} \\
\hline \multicolumn{7}{|l|}{13} \\
\hline 14 None & $\$ 0.00$ & $\$ 0.00$ & $\$ 0.00$ & $\$ 0.00$ & $\$ 0.00$ & $\$ 0.00$ \\
\hline 15 & $\$ 0.00$ & $\$ 0.00$ & $\$ 0.00$ & 50.00 & $\$ 0.00$ & $\$ 0.00$ \\
\hline \multicolumn{7}{|l|}{16} \\
\hline 17 Subtotal & $\$ 958.45$ & $\$ 6.85$ & $\$ 391.82$ & $\$ 7.58$ & $\$ 519.27$ & $\$ 4.82$ \\
\hline 18 Markup (15\%) & $\$ 143.77$ & $\$ 1.03$ & $\$ 58.77$ & $\$ 1.14$ & $\$ 77.89$ & $\$ 0.72$ \\
\hline 19 Total & $\$ 1,102.22$ & $\$ 7.88$ & $\$ 450.59$ & $\$ 8.72$ & $\$ 597.16$ & $\$ 5.55$ \\
\hline
\end{tabular}

Table 6. Detailed breakdown of Option 3 cost calculations.

TABLE NOTES:

1. Ballasted roof structure. Uses $\$ 1.00 / \mathrm{W}$ for crystalline installation $(\$ 140 / \mathrm{m} 2)$. Source: AD Little 6/94.

6. PV Wiring. $\$ 37.67 / \mathrm{m} 2(\$ 3.50 / \mathrm{sf})$ PV area allowance. Could be significantly less: assuming modules are connected in series of 6 (a-Si modules $140 \mathrm{~V}$ ea for a $240 \mathrm{~V}$ system) with snap together connectors in the series string, conduit is needed only for wiring between strings and for home runs to the inverter. In the test building, this should require approx. $1 \mathrm{~m}$ conduit $/ 10 \mathrm{~m} 2 \mathrm{PV}$ area, or $\$ 10.00 / \mathrm{m} 2 @ \$ 10 / \mathrm{m}$ wiring cost allowance.

7. Other indirect. Engineering, permits, shipping, insurance, project management. Source: $A D$ Little 6/94.

8. Power conditioning. Source: various industry reports. This figure should be conservative for a system of over $100 \mathrm{~kW}$. APS-Fairfield data suggest a cost of under $\$ 0.60 / \mathrm{W}$ for a system this size. 


\section{SECTION}

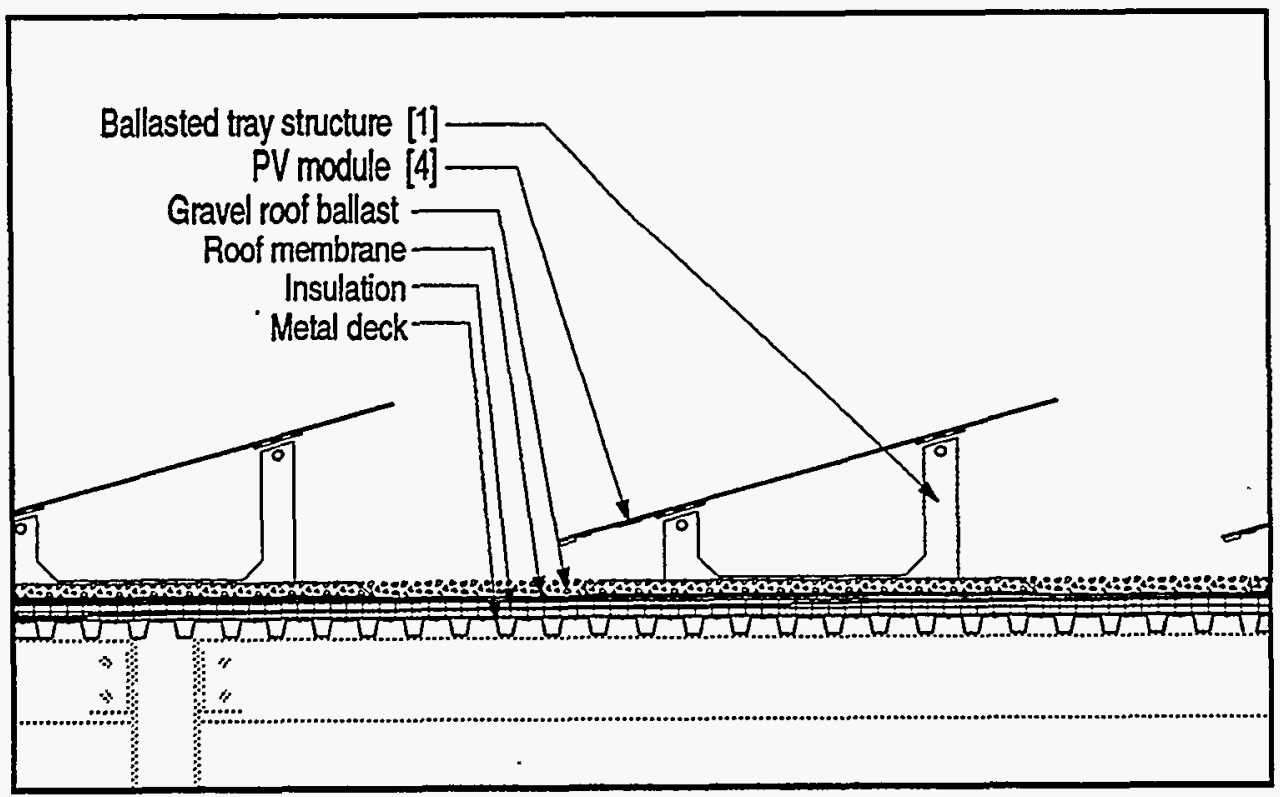

Fig 27. Section through typical bay, Option 3 


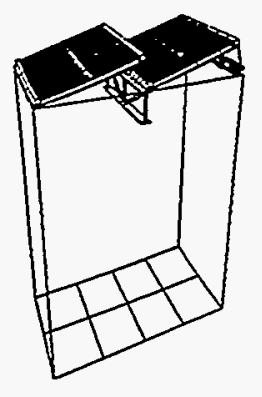

\section{OPTION 4 CONSTRUCTION COSTS}

\begin{tabular}{|c|c|c|c|c|c|c|}
\hline & $\mathrm{s} / \mathrm{m} 2$ & $\begin{array}{l}\text { PV1 } \\
\text { S/W }\end{array}$ & $\$ / m 2$ & $\begin{array}{c}\text { PV2 } \\
\text { S/W }\end{array}$ & $\$ / m 2$ & $\begin{array}{r}\text { PV } \\
\text { S/W } \\
\end{array}$ \\
\hline $\begin{array}{l}1 \text { Additional roof structure } \\
2 \\
3 \text { PV system costs: }\end{array}$ & $\$ 32.28$ & S0.23 & $\$ 32.28$ & $\$ 0.62$ & $\$ 32.28$ & $\$ 0.30$ \\
\hline 4 PV modules & $\$ 615.72$ & $\$ 4.40$ & $\$ 155.01$ & $\$ 3.00$ & $\$ 215.29$ & $\$ 2.00$ \\
\hline 5 Module subframe & $\$ 62.43$ & S0.45 & $\$ 62.43$ & $\$ 1.21$ & $\$ 62.43$ & $\$ 0.58$ \\
\hline 6 & $\$ 0.00$ & $\$ 0.00$ & $\$ 0.00$ & $\$ 0.00$ & $\$ 0.00$ & $\$ 0.00$ \\
\hline 7 PV wiring & $\$ 34.88$ & So.25 & $\$ 34.88$ & $\$ 0.68$ & $\$ 34.88$ & $\$ 0.32$ \\
\hline 8 Other indirect & $\$ 55.97$ & $\$ 0.40$ & $\$ 20.67$ & $\$ 0.40$ & $\$ 43.06$ & $\$ 0.40$ \\
\hline 9 Clerestory Glazing & $\$ 77.50$ & S0.55 & $\$ 77.50$ & $\$ 1.50$ & $\$ 77.50$ & $\$ 0.72$ \\
\hline 10 Add. sprinklers & $\$ 9.15$ & $\$ 0.07$ & $\$ 9.15$ & $\$ 0.18$ & $\$ 9.15$ & $\$ 0.09$ \\
\hline 11 Power Conditioning & $\$ 111.95$ & $\$ 0.80$ & $\$ 41.33$ & $\$ 0.80$ & $\$ 86.11$ & $\$ 0.80$ \\
\hline $\begin{array}{l}12 \\
13 \text { Material credits: } \\
14\end{array}$ & $\$ 999.88$ & $\$ 7.15$ & $\$ 433.25$ & $\$ 8.39$ & $\$ 560.70$ & $\$ 5.21$ \\
\hline 15 None & 50.00 & 50.00 & $\$ 0.00$ & $\$ 0.00$ & $\$ 0.00$ & $\$ 0.00$ \\
\hline 16 & $\$ 0.00$ & $\$ 0.00$ & $\$ 0.00$ & $\$ 0.00$ & $\$ 0.00$ & $\$ 0.00$ \\
\hline 17 Subtotal & $\$ 999.88$ & $\$ 7.15$ & $\$ 433.25$ & $\$ 8.39$ & $\$ 560.70$ & $\$ 5.21$ \\
\hline 18 Markup (15\%) & $\$ 149.98$ & $\$ 1.07$ & $\$ 64.99$ & $\$ 1.26$ & $\$ 84.10$ & 50.78 \\
\hline 19 Total: All & $\mathbf{S 1 , 1 4 9 . 8 6}$ & $\$ 8.22$ & $\$ 498.24$ & $\$ 9.64$ & $\$ 644.80$ & $\$ 5.99$ \\
\hline 20 Total:PV only & $\$ 1,013.09$ & $\$ 7.24$ & $\$ 361.46$ & $\$ 7.00$ & $\$ 508.03$ & $\$ 4.72$ \\
\hline 21 Total:Daylightonly & $\$ 136.77$ & NA & $\$ 136.77$ & NA & $\$ 136.77$ & NA \\
\hline
\end{tabular}

Table 7. Detailed breakdown of Option 4 cost calculations.

TABLE NOTES:

1. Additional roof structure. tube steel framing to form sawtooth roof profile.Source: Devcon Construction Inc., 1994.

5. Module subframe. Steel channel support structure for $2^{\prime \prime}-6^{\prime \prime} \times 5^{\prime}-0^{\prime \prime}$ modules. Source: Bell Products.

7. PV wiring. $\$ 37.67 / \mathrm{m} 2$ ( $\$ 3.50 / \mathrm{sf}$ ) PV area allowance. Could be significantly less: assuming modules are connected in series of six (a-Si modules @ $40 \mathrm{~V}$ ea for a $240 \mathrm{~V}$ system) with snap together connectors in the series string, conduit is needed only for wiring between strings and for home runs to the inverter. In the test building, this should require approx. $1 \mathrm{~m}$ conduit $/ 10 \mathrm{~m} 2 \mathrm{PV}$ area, or $\$ 10.00 / \mathrm{m} 2 @ \$ 10 / \mathrm{m}$ wiring cost allowance.

8. Other indirect. Engineering, permits, shipping, insurance, project management. Source: AD Little report $6 / 94$.

9. Clerestory glazing. Insulated glazing at $\$ 344 / \mathrm{m} 2$ for clerestory area. Source: Devcon Construction Inc., 1994.

10. Additional sprinklers. Sprinkler heads required to cover volumes of sawtooth roof. Source: Devcon Construction Inc., 1994.

11. Power conditioning. This figure should be conservative for a system of over $100 \mathrm{~kW}$. APS-Fairfield data suggest a cost of under $\$ 0.60 / \mathrm{W}$ for a system this size.

20 PV Only. Sum of lines: $4+5+7+8+11$.

21 Daylight Only. Sum of lines: $1+9+10+14+15$. 


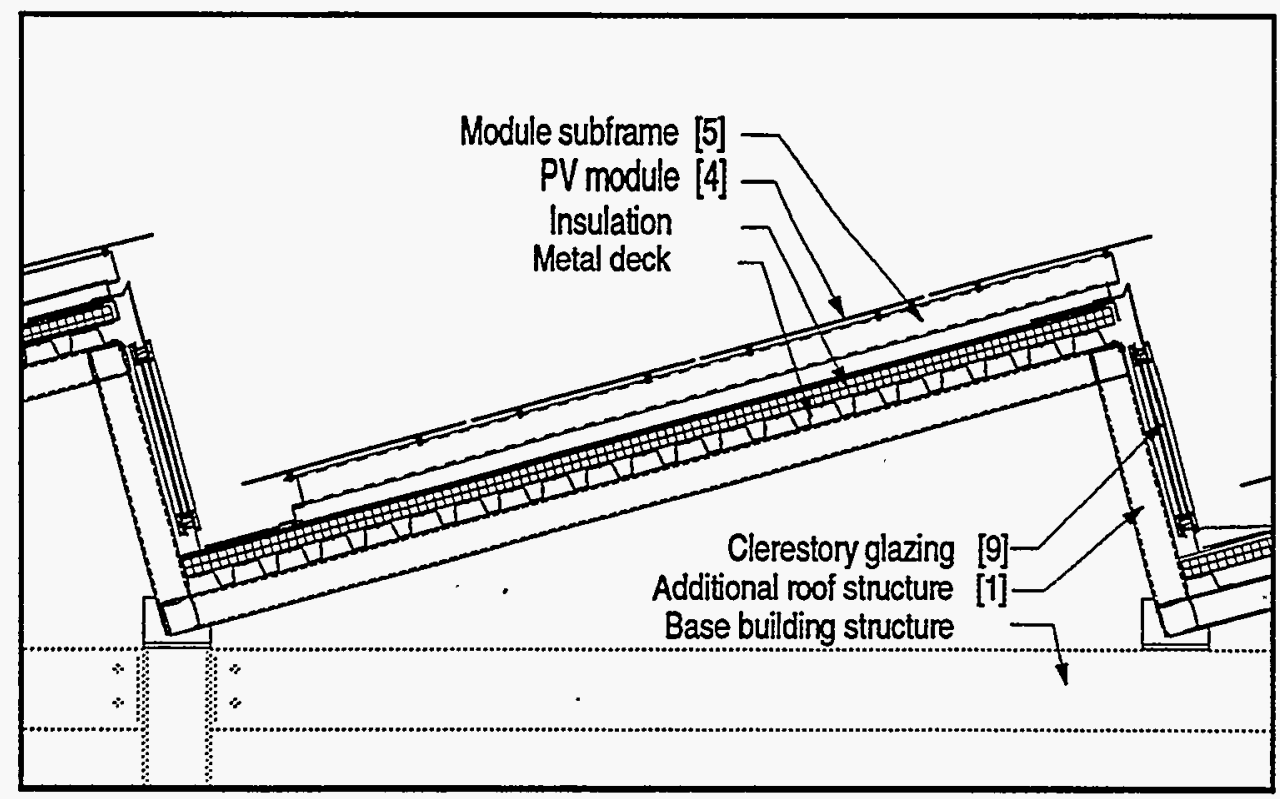

Fig 28. Section through typical bay, Option 4 


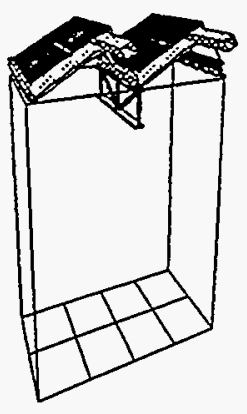

\section{OPTION 5 CONSTRUCTION COSTS}

\begin{tabular}{|c|c|c|c|c|c|c|}
\hline & \multicolumn{2}{|r|}{ PV1 } & \multicolumn{2}{|r|}{ PV2 } & \multicolumn{2}{|r|}{$\overline{\text { PV3 }}$} \\
\hline & $\mathrm{s} / \mathrm{m} 2$ & $S / W$ & $\mathrm{~s} / \mathrm{m} 2$ & S/W & $\mathrm{s} / \mathrm{m} 2$ & $S N$ \\
\hline $\begin{array}{l}1 \text { Additional roof structure } \\
2 \\
3 \text { PV system costs: }\end{array}$ & $\$ 32.28$ & $\$ 0.23$ & $\$ 32.28$ & $\$ 0.62$ & $\$ 32.28$ & $\$ 0.30$ \\
\hline 4 PV modules & $\$ 615.72$ & $\$ 4.40$ & $\$ 155.01$ & $\$ 3.00$ & $\$ 215.29$ & $\$ 2.00$ \\
\hline $5 \mathrm{PV}$ framing & $\$ 215.29$ & $\$ 1.54$ & $\$ 215.29$ & $\$ 4.17$ & $\$ 215.29$ & $\$ 2.00$ \\
\hline 6 & $\$ 0.00$ & $\$ 0.00$ & $\$ 0.00$ & $\$ 0.00$ & $\$ 0.00$ & $\$ 0.00$ \\
\hline 7 PV wiring & $\$ 34.88$ & $\$ 0.25$ & $\$ 34.88$ & $\$ 0.68$ & $\$ 34.88$ & $\$ 0.32$ \\
\hline 8 Other indirect & $\$ 55.97$ & $\$ 0.40$ & $\$ 20.67$ & $\$ 0.40$ & $\$ 43.06$ & $\$ 0.40$ \\
\hline 9 Clerestory Glazing & $\$ 77.50$ & $\$ 0.55$ & $\$ 77.50$ & $\$ 1.50$ & $\$ 77.50$ & $\$ 0.72$ \\
\hline 10 Liner Panel & $\$ 21.53$ & \$0.15 & $\$ 21.53$ & \$0.42 & $\$ 21.53$ & $\$ 0.20$ \\
\hline 11 Heat recovery, therm. stor. & $\$ 64.59$ & \$0.46 & $\$ 64.59$ & $\$ 1.25$ & $\$ 64.59$ & $\$ 0.60$ \\
\hline 12 Add. sprinklers & $\$ 9.15$ & $\$ 0.07$ & $\$ 9.15$ & $\$ 0.18$ & $\$ 9.15$ & $\$ 0.09$ \\
\hline 13 Power Conditioning & $\$ 111.95$ & $\$ 0.80$ & $\$ 41.33$ & $\$ 0.80$ & $\$ 86.11$ & $\$ 0.80$ \\
\hline 14 & $\$ 1,238.85$ & $\$$ & $\$ 672.21$ & $\$ 13.01$ & $\$ 799.66$ & $\$ 7.43$ \\
\hline 15 Material credits: & & & & & & \\
\hline 16 Roofing & $\$ 0.00$ & $\$ 0.00$ & $\$ 0.00$ & $\$ 0.00$ & $\$ 0.00$ & $\$ 0.00$ \\
\hline 17 Metal Deck & $\$ 0.00$ & $\$ 0.00$ & 50.00 & $\$ 0.00$ & $\$ 0.00$ & $\$ 0.00$ \\
\hline 18 & 50.00 & $\$ 0.00$ & 50.00 & 50.00 & $\$ 0.00$ & $\$ 0.00$ \\
\hline 19 Subtotal & $\$ 1,238.85$ & $\$ 8.85$ & $\$ 672.21$ & $\$ 13.01$ & $\$ 799.66$ & $\$ 7.43$ \\
\hline 20 Markup (15\%) & $\$ 185.83$ & $\$ 1.33$ & $\$ 100.83$ & $\$ 1.95$ & $\$ 119.95$ & $\$ 1.11$ \\
\hline 21 Total:All & $\$ 1,424.67$ & $\$ 10.18$ & $\$ \mathbf{\$ 7 7 3 . 0 5}$ & \$14.96 & \$919.61 & $\$ 8.54$ \\
\hline 20 Total:PV only & $\$ 1,188.87$ & $\$ 8.50$ & $\$ 537.24$ & $\$ 10.40$ & $\$ 683.81$ & $\$ 6.35$ \\
\hline 21 Total:Daylloht + Themal & $\$ 235.80$ & NA & $\$ 235.80$ & NA & $\$ 235.80$ & NA \\
\hline
\end{tabular}

Table 8. Detailed breakdown of Option 5 cost calculations.

\section{TABLE NOTES:}

1. Additional roof structure. tube steel framing to form sawtooth roof profile. Source: Deocon Construction Inc, 1994.

5: PV framing. Aluminum extrusion skylight system. Source: DeaMor, Inc. 1994

7. PV wiring. $\$ 37.67 / \mathrm{m} 2$ ( $\$ 3.50 / \mathrm{sf}) \mathrm{PV}$ area allowance. Could be significantly less: assuming modules are connected in series of six (a-Si modules @ $40 \mathrm{~V}$ ea for a $240 \mathrm{~V}$ system) with snap together connectors in the series string, conduit is needed only for wiring between strings and for home runs to the inverter. In the test building, this should require approx. $1 \mathrm{~m}$ conduit $/ 10 \mathrm{~m} 2 \mathrm{PV}$ area, or $\$ 10.00 / \mathrm{m} 2 @ \$ 10 / \mathrm{m}$ wiring cost allowance.

8. Other indirect. Engineering, permits, shipping, insurance, project management. Source: $A D$ Little report 6/94

9. Clerestory glazing. Insulated glazing at $\$ 344 / \mathrm{m} 2$ for clerestory area. Source: Devcon Construction Inc., 1994.

10. Liner panel. Cost of plywood liner to create airspace behind PV modules.

11. Heat recovery HVAC, thermal storage. Budget price for ductwork, controls, thermal mass storage system. Source: Ove Arup \& Partners New York

12. Additional sprinklers. Sprinkler heads required to cover volumes of sawtooth roof. Source: Deocon Construction Inc., 1994.

13. Power conditioning. This figure should be conservative for a system of over $100 \mathrm{~kW}$. APS-Fairfield data suggest a cost of under $\$ 0.60 / \mathrm{W}$ for a system this size.

20 PV Only. Sum of lines: $4+5+7+8+11$.

21 Daylight +Thermal. Sum of lines: $1+9+10+14+15$. 


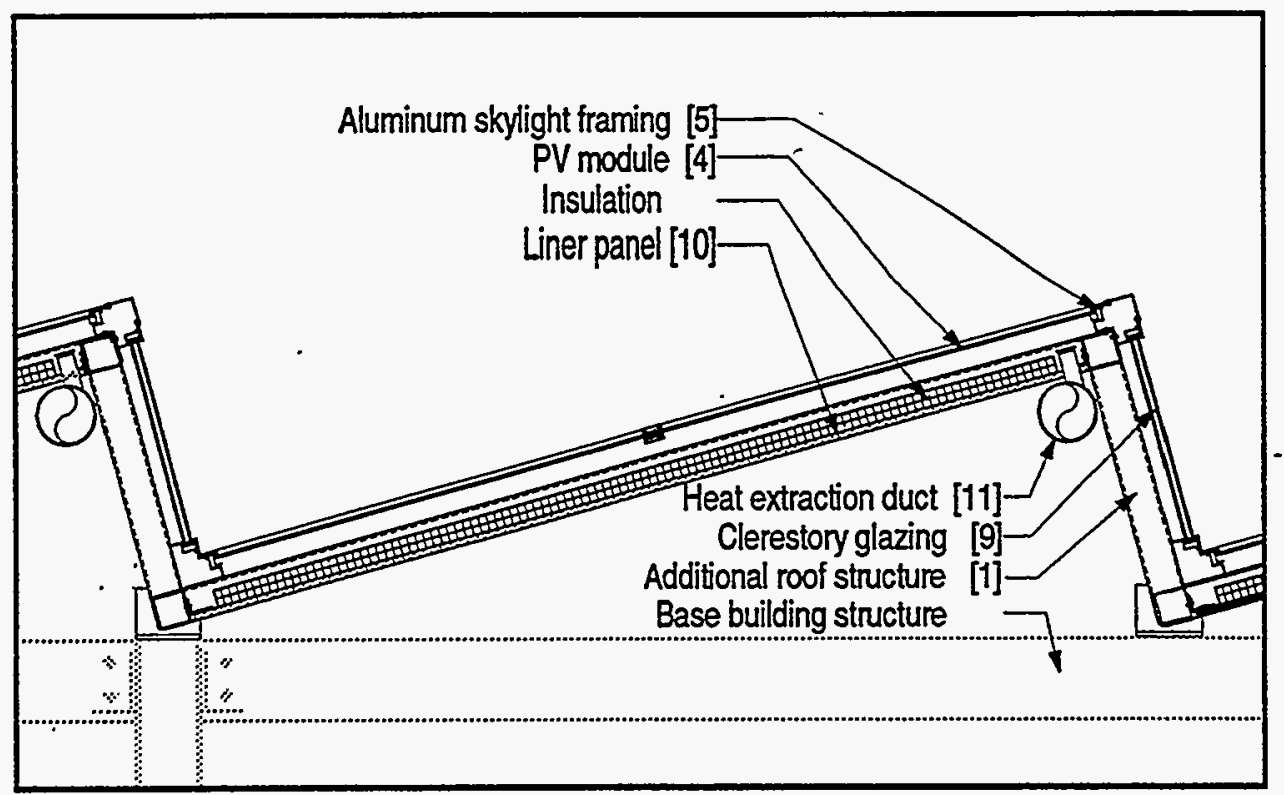

Fig 29. Section through typical bay, Option 5 


\section{UTILITY RATE ANALYSIS}

To obtain a payback period for each system, the costs obtained in the preceding section are divided by the value of energy savings and PV income. Since the thermal performance and PV output of each system was determined for each of the six test cities, the actual value of PV electricity was calculated for each location and used as the basis for the payback calculation. (see Appendix D for detailed calculations of average utility rates.)

We used utility rate data compiled by Casazza, Schultz \& Associates for the Gas Research Institute, entitled "Electric and Gas Rates for the Residential, Commercial and Industrial Sectors: 1993". In each location a rate was found which was applicable to a commercial customer with a peak demand of $5500 \mathrm{~kW}$. When there was more than one applicable rate, the rate that appeared to yield the best return based on maximum time-of-use energy rates were selected (we did not evaluate every rate available).

One exceptional rate was evaluated, a LILCO small commercial $(<7 \mathrm{~kW}$ demand) rate in New York. This rate (noted as New York 2) provides exceptionally high peak energy charges, and gives an idea of the high end of the rates smaller commercial or residential users might find in certain parts of the country.

To obtain the most accurate value of avoided electricity, PV performance was modeled on an hourly basis for each climate using PV-F Chart software. The PV production for each hour of the day for each month was multiplied by the electric rate prevailing at that hour, taking into account time-of-use charges, demand charges, seasonal variations, surcharges, energy cost adjustments and taxes. Demand charges were offset by $20 \%$ of the capacity of the PV system, which would be minimum output for most PV systems during cloudy days. The total cost of electricity for the year was then divided by the total kilowatthours produced by the PVs to obtain the average offset electric cost.

These evaluations were performed for three different array orientations: South, $45^{\circ}$ West of South, and West. (see "PV Orientation Effects on Average Electric Rate" below.) 


\section{DEMAND CHARGE ANALYSIS}

Table 9 summarizes the average rate in $\$ / \mathrm{kWh}$ for four different levels of $\mathrm{PV}$ demand credit. Note that the Oakland and New York 2 rates are energy charges only and have no demand component.

\begin{tabular}{|lrrrr|}
\hline & & \multicolumn{4}{c|}{$\%$ Demand Credit } \\
\cline { 3 - 5 } & $0 \%$ & $20 \%$ & $60 \%$ & $100 \%$ \\
Oakland & $\$ 0.1753$ & $\$ 0.1753$ & $\$ 0.1753$ & $\$ 0.1753$ \\
New York1 & $\$ 0.1258$ & $\$ 0.1306$ & $\$ 0.1402$ & $\$ 0.1499$ \\
New York2 & $\$ 0.2727$ & $\$ 0.2727$ & $\$ 0.2727$ & $\$ 0.2727$ \\
Phoenix & $\$ 0.0878$ & $\$ 0.0897$ & $\$ 0.0935$ & $\$ 0.0972$ \\
Miami & $\$ 0.1091$ & $\$ 0.1158$ & $\$ 0.1291$ & $\$ 0.1424$ \\
Chicago & $\$ 0.0604$ & $\$ 0.0727$ & $\$ 0.0975$ & $\$ 0.1223$ \\
Cincinnati & $\$ 0.0470$ & $\$ 0.0561$ & $\$ 0.0742$ & $\$ 0.0924$ \\
\hline
\end{tabular}

Table 9. PV demand credit levels for different locations.

Although it is not in the scope of this report, there may be cases where storage to achieve greater demand savings is cost-effective. In Chicago, for example, the effective value of PV electricity is more than doubled by capturing $100 \%$ demand credit. The additional value is $\$ 0.0619 / \mathrm{kWh} /$ year.

Almost all the commercial utility rates we examined had a demand charge component. Energy-charge-only rates are common in residential rate plans, less common in small commercial rates, and rare in medium-large commercial rates. In general, the lower the demand charge component of a rate, the higher the value of $P V$ power. 


\section{PV ORIENTATION EFFECTS ON AVERAGE ELECTRIC RATE}

The following three charts illustrate the effects of array orientation on PV electric value. Although annual PV output from a West-facing array $\left(90^{\circ}\right)$ was typically lower than output from a South-facing and Southwest-facing array (Chart 7 ), a $90^{\circ}$ orientation produced an equivalent or better overall electric rate (Chart 8) as a result of higher utility rates in the latter part of the day. The average annual PV value was calculated by multiplying the average electric rate by the PV power produced (Chart 9). The payback analyses which follow use the $45^{\circ}$ orientation for producing the best overall income.

Detailed PV output and electrical rate calculations are listed in Appendix D.

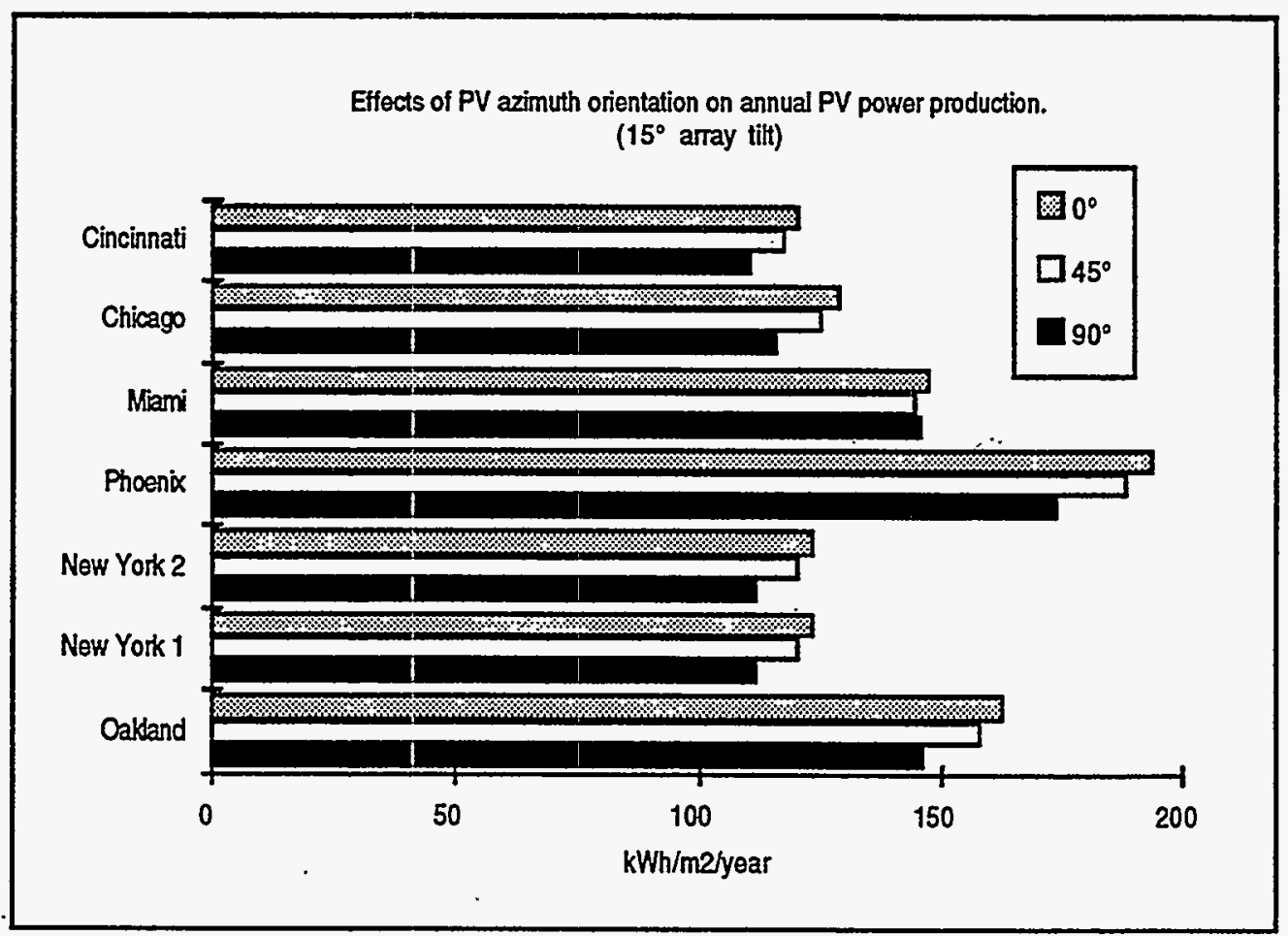

Chart 7. Annual PV power production for three different array orientations. 
Effects of PV azimuth orientation on AVERAGE VALUE of PV electricity. (15 array tit)

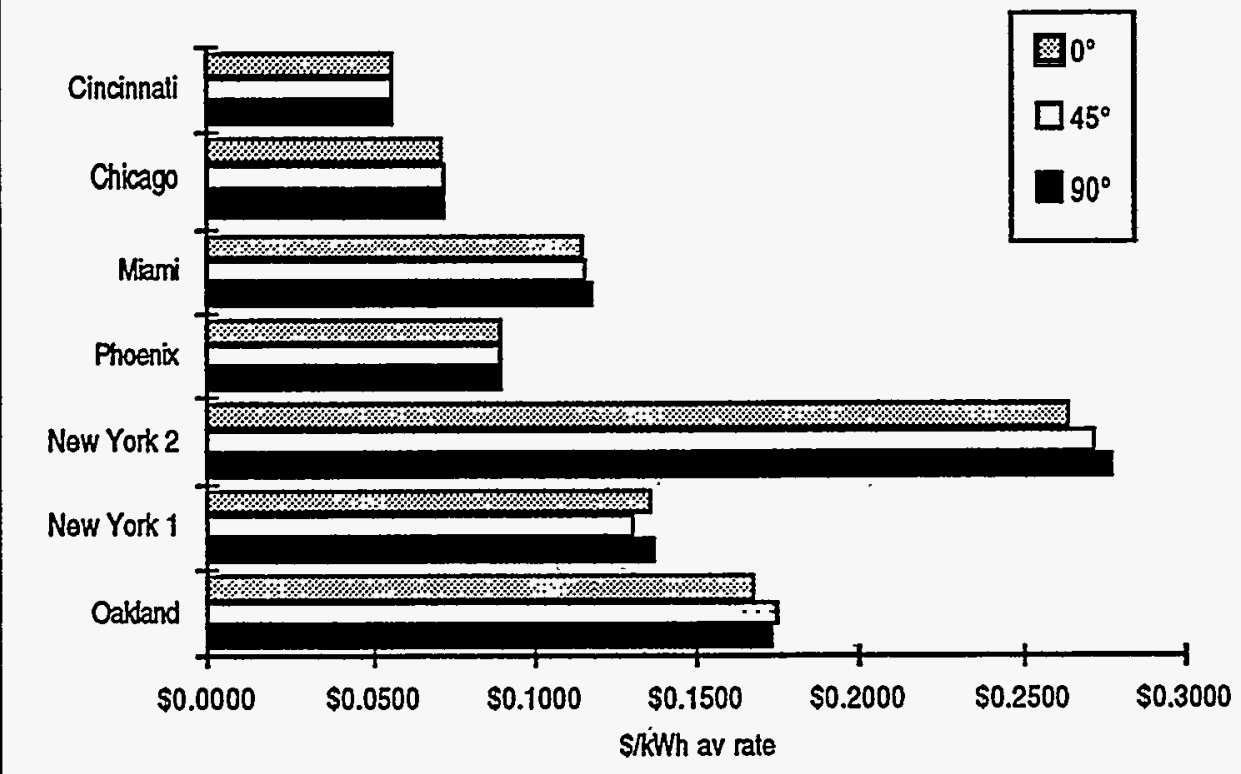

Chart 8. Average PV electric value for three different array orientations.

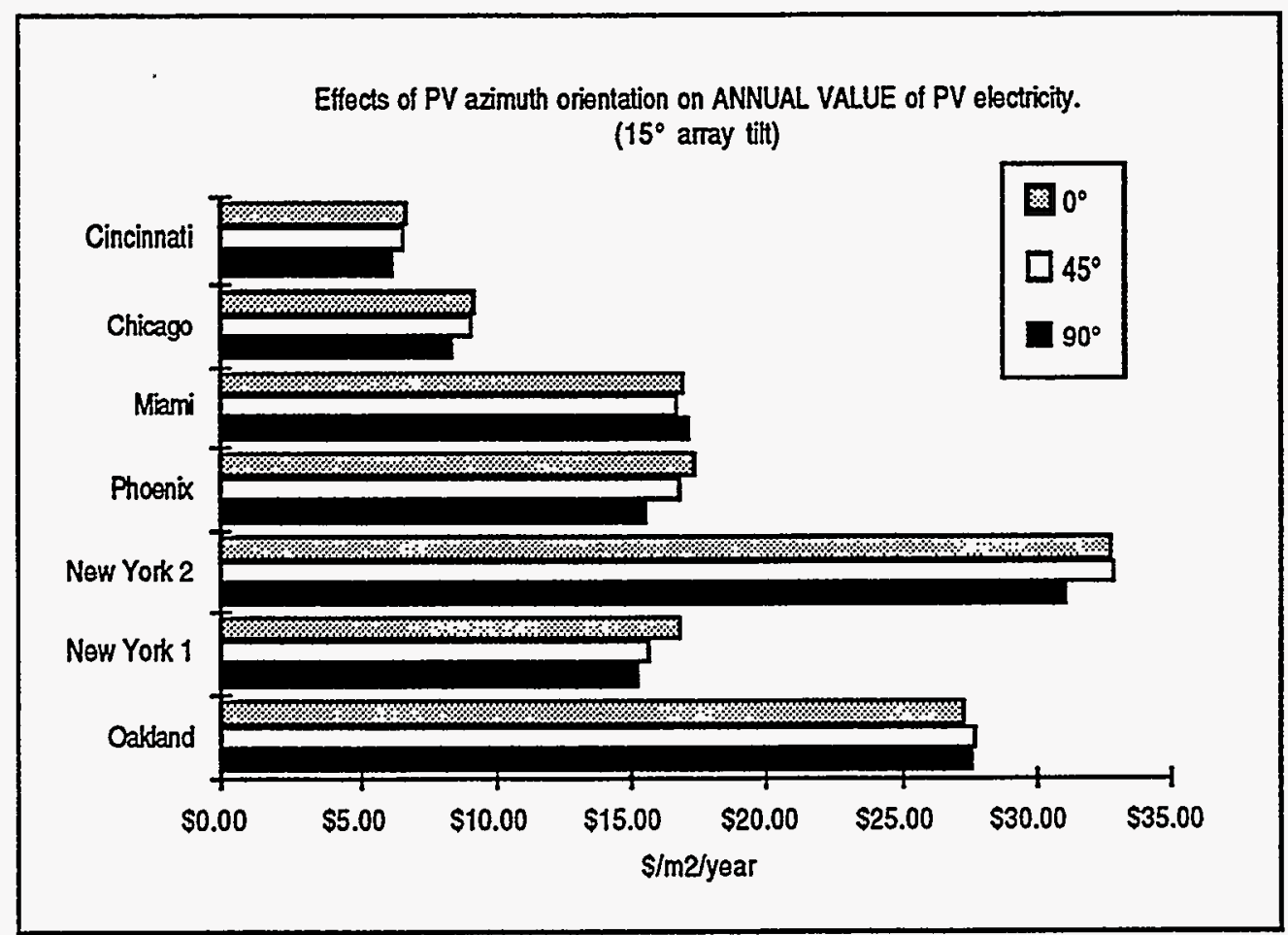

Chart 9. Annual PV value (PV power $x$ average PV electric value) for three different array orientations. 


\section{PAYBACK ANALYSIS - PARAMETERS}

To assess the relative economic value of the options, a simple payback was calculated for each system in each climate for the three PV technologies. For Options 4 and 5, a PV system-only payback was calculated since the daylight/thermal benefits of these designs are not an integral product of the PV modules. The payback calculations are:

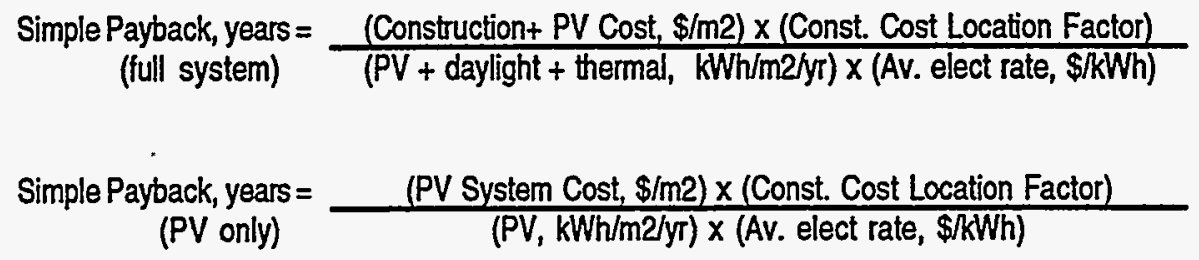

More sophisticated economic models would give a better picture of the actual value of the systems analyzed. We made no attempt to include life cycle costs such as maintenance, cleaning, insurance or any other additional operating costs. We also did not attempt present value calculations.

Some other assumptions are worth noting:

1. Some of the system costs may be high for a project of this size (see cost calculations section, particularly in regard to power conditioning and wiring costs), although the markup of $15 \%$ for the PV system costs may be higher in cases with multiple subcontractors.

2. No escalation was calculated for electric rates.

3. A demand credit of $20 \%$ was given for the PV system. In some cases, a higher demand credit may apply.

4. Tax credits were not factored into payback. For corporate building owners with tax liabilities, these savings could substantially cut the effective cost of the PV systems. A $10 \%$ federal renewable energy tax credit, combined with an accelerated five year depreciation of the system costs, can cut the system cost by more than $40 \%$. Other state and Federal credits and . subsidies may apply.

5. The effects of incorporating PV system costs into a building mortgage have not been considered.

The overall effect of these factors could cut the payback times shown by half or more. 


\section{PAYBACK ANALYSIS - SUMMARY}

Tables 10 and 11 summarize the simple payback period calculated in years for each of the systems. For each option, the best payback of the two currently available PV technologies (PV1 and PV2) is highlighted in boldface.

\begin{tabular}{|c|c|c|c|c|c|c|c|c|}
\hline \multicolumn{9}{|c|}{ Payback,PVOnly } \\
\hline & & Oakland & New York 1 & New York 2 & Phoenix & Miami & Chicago & Cincinnati \\
\hline \multirow[t]{3}{*}{ OPTION 1} & $P V 1$ & 35.68 & 72.65 & 34.79 & 47.15 & 45.74 & 100.54 & 124.02 \\
\hline & PV2 & 36.84 & 75.38 & 36.10 & 47.84 & 46.54 & 104.32 & 128.55 \\
\hline & PV3 & 25.30 & 51.63 & 24.72 & 33.14 & 32.19 & 71.46 & 88.10 \\
\hline \multirow[t]{3}{*}{ OPTION 1a } & $\overline{P V 1}$ & 27.62 & 56.24 & 26.93 & 36.50 & 35.41 & 77.82 & 96.00 \\
\hline & PV2 & 16.82 & 34.41 & 16.48 & 21.84 & 21.25 & 47.62 & 58.68 \\
\hline & PV3 & 14.85 & 30.31 & 14.51 & 19.45 & 18.90 & 41.95 & 51.71 \\
\hline \multirow[t]{3}{*}{ OPTION 2} & $P V 1$ & 43.30 & 87.99 & $\overline{42.14}$ & 57.30 & 55.52 & 121.84 & 150.26 \\
\hline & PV2 & 54.96 & 112.31 & 53.78 & 71.43 & 69.44 & 155.51 & 191.59 \\
\hline & PV3 & 34.86 & 71.04 & 34.02 & 45.72 & 44.37 & 98.37 & 121.25 \\
\hline \multirow[t]{3}{*}{ OPTION 2a } & $P V 1$ & 29.05 & 59.05 & 28.27 & 38.45 & 37.25 & 81.76 & 100.83 \\
\hline & PV2 & 19.72 & 40.30 & 19.30 & 25.63 & 24.92 & 55.80 & 68.74 \\
\hline & PV3 & 16.44 & 33.51 & 16.04 & 21.56 & 20.92 & 46.39 & 57.18 \\
\hline \multirow[t]{3}{*}{ OPTION 3} & PV1 & 34.74 & 70.92 & 33.96 & 45.83 & 44.53 & 98.07 & 121.01 \\
\hline & PV2 & 35.44 & 72.63 & 34.78 & 45.97 & 44.77 & 100.47 & 123.82 \\
\hline & PV3 & 24.45 & 50.00 & 23.94 & 31.98 & 31.11 & 69.15 & 85.28 \\
\hline \multirow[t]{3}{*}{ OPTION 4} & $P V 1$ & 33.74 & 68.24 & 32.68 & 44.82 & 43.29 & 94.63 & 116.63 \\
\hline & PV2 & 29.51 & 60.11 & 28.78 & 38.43 & 37.28 & 83.31 & 102.59 \\
\hline & PV3 & 21.78 & 44.20 & 21.17 & 28.64 & 27.73 & 61.29 & 75.50 \\
\hline \multirow[t]{3}{*}{ OPTION 5,5a } & $P V 1$ & 37.62 & 76.74 & 36.75 & 49.64 & 48.21 & 106.13 & 130.95 \\
\hline & PV2 & 42.36 & 86.78 & 41.56 & 54.97 & 53.52 & 120.06 & 147.97 \\
\hline & PV3 & 28.08 & 57.41 & 27.49 & 36.75 & 35.73 & 79.41 & 97.92 \\
\hline
\end{tabular}

Table 10 A summary matrix of simple payback periods for all possible $P V$ roof option, $P V$ system cost/benefits only (See Appendix D for detailed payback tables for each option). 


\begin{tabular}{|lcrrrrrrr|}
\hline Payback, Fullsystem & & & & & & \\
& \multicolumn{2}{c}{ Cakjand } & New York1 & New York2 & Phoenix & Mami & Chicago & Cincinnati \\
\hline OPTION1 & PV1 & 52.55 & 295.81 & 141.65 & negative & negative & 533.09 & $2,426.02$ \\
& PV2 & 138.10 & negative & negative & negative & negative & negative & negative \\
& PV3 & 44.09 & $1,280.41$ & 613.14 & negative & negative & negative & negative \\
\hline OPTION 1a & PV1 & 27.62 & 56.24 & 26.93 & 36.50 & 35.41 & 77.82 & 96.00 \\
& PV2 & 16.82 & 34.41 & 16.48 & 21.84 & 21.25 & 47.62 & 58.68 \\
& PV3 & 14.85 & 30.31 & 14.51 & 19.45 & 18.90 & 41.95 & 51.71 \\
\hline OPTION 2 & PV1 & 35.29 & 74.43 & 37.56 & 61.91 & 53.37 & 110.12 & 137.67 \\
& PV2 & 35.19 & 77.20 & 40.80 & 85.31 & 62.81 & 122.57 & 155.50 \\
& PV3 & 27.03 & 57.75 & 30.03 & 50.97 & 42.45 & 86.87 & 108.98 \\
\hline OPTION 2a & PV1 & 29.05 & 59.05 & 28.27 & 38.45 & 37.25 & 81.76 & 100.83 \\
& PV2 & 19.72 & 40.30 & 19.30 & 25.63 & 24.92 & 55.80 & 68.74 \\
& PV3 & 16.44 & 33.51 & 16.04 & 21.56 & 20.92 & 46.39 & 57.18 \\
\hline OPTION 3 & PV1 & 34.67 & 70.67 & 33.84 & 45.60 & 44.30 & 97.74 & 120.50 \\
& PV2 & 35.25 & 71.98 & 34.47 & 45.41 & 44.20 & 99.63 & 122.53 \\
& PV3 & 24.38 & 49.77 & 23.83 & 31.77 & 30.90 & 68.85 & 84.81 \\
\hline OPTION 4 & PV1 & 25.37 & 47.70 & 22.84 & 35.52 & 31.74 & 69.86 & 83.79 \\
& PV2 & 17.92 & 32.34 & 15.49 & 25.86 & 21.97 & 48.98 & 57.74 \\
& PV3 & 16.55 & 30.85 & 14.77 & 23.33 & 20.58 & 45.59 & 54.42 \\
\hline OPTION 5 & PV1 & 33.85 & 59.57 & 28.53 & 45.42 & 42.79 & 84.53 & 105.38 \\
& PV2 & 33.36 & 52.80 & 25.28 & 44.97 & 41.37 & 76.22 & 95.63 \\
& PV3 & 26.30 & 45.07 & 21.58 & 35.26 & 33.01 & 64.26 & 80.24 \\
\hline
\end{tabular}

Table 11 A summary matrix of simple payback periods for all possible PV roof options, full system cost/benefits. (See Appendix D for detailed payback tables for each option). 


\section{PAYBACK ANALYSIS-DISCUSSION}

Payback period is a function of a large number of variables, some specific to each roof option, some to location, some to PV technology. Depending on the project, it may be necessary to evaluate a BIPV installation according to one or more of these criteria:

1. Location

PV output (insolation)

Electrical rate

Construction cost location factor

Building energy requirements (climate)

2. BIPV Option

Cost (Construction cost minus material credit)

Thermal performance

Daylight performance

PV performance (a function of PV module temperature)

3. PV technology

Cost $(\$ / W)$

Efficiency $\left(W / m^{2}\right)$

Temperature coefficient $\left(\%\right.$ efficiency $/{ }^{\circ} \mathrm{C}$ )

\section{LOCATION}

Of the three categories, location has the greatest effect on payback, with the best area averaging more than 3.5 times better than the worst. The effect of location on payback is consistent for each BIPV system: Oakland always has the shortest payback, followed by Miami, Phoenix, New York, Chicago, and Cincinnati (see Chart 10). As discussed in the previous section, we included one exceptional case: in New York, a time-of-use rate for small commercial customers with demand too small to qualify for our reference building (less than $7 \mathrm{kWp}$ ). Using this rate, the shortest payback of all is achieved, despite the lowest insolation and the highest construction costs.

In general, Oakland has a favorable balance of mild climate, high electrical rates, and high insolation. Phoenix, which has the highest insolation by far, has a hot climate and moderate electrical rates which lengthen the payback periods. At the other end of the range, Cincinnati has the lowest electrical rate, the second-lowest insolation, and significant heating and cooling seasons. 


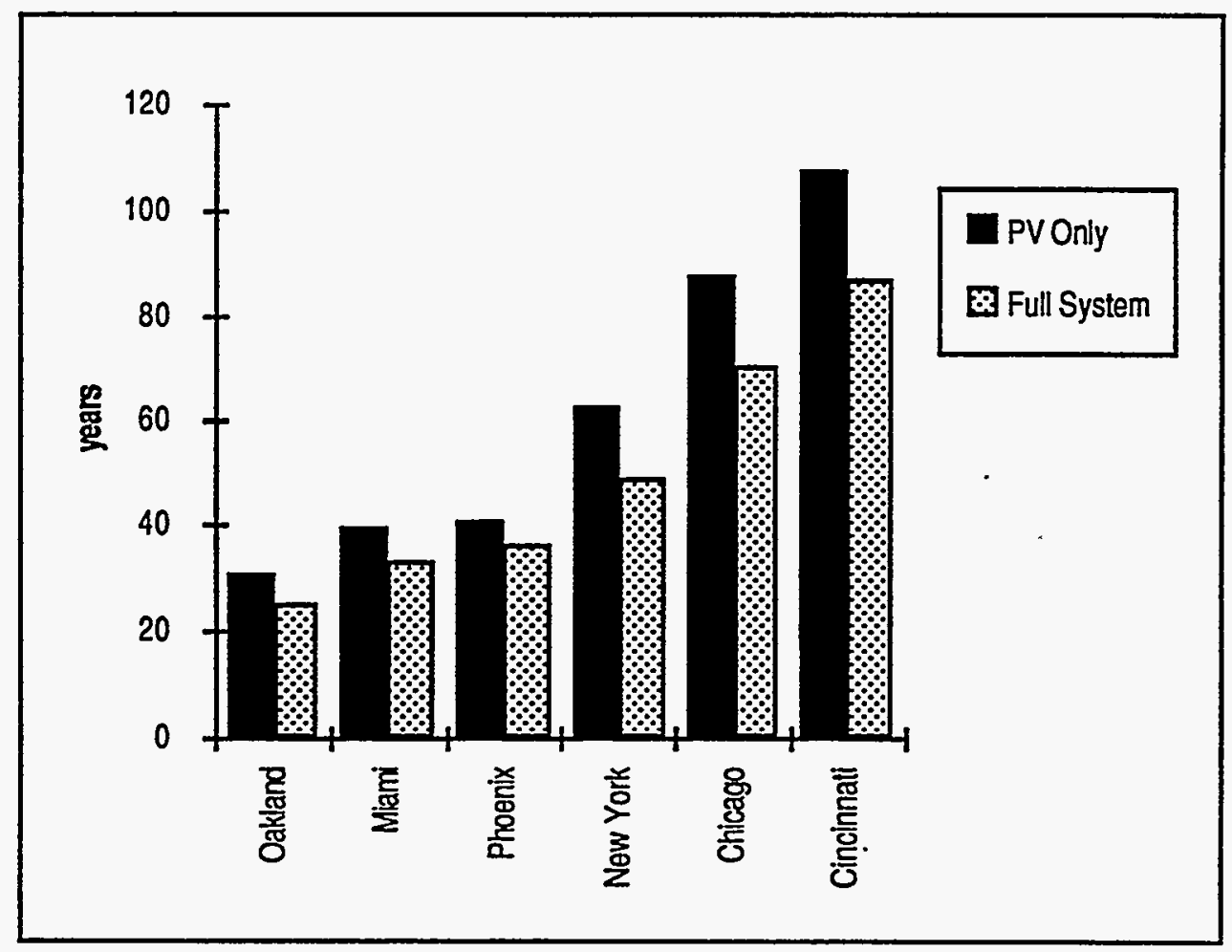

Chart 10. Average payback by location, all options, all PV technologies.

It is important to note that the paybacks shown in Chart 10 are averages for all BIPV options and PV technologies. In each location, the shortest payback achieved was approximately $60 \%$ of the average. Given any significant tax savings related to the PV system (see above, p. 44), full system payback periods of less than 20 years could be possible in all the locations except Chicago and Cincinnati.

\section{BIPV OPTION}

The pattern is not as consistent when payback is evaluated according to BIPV roof option. The range between the best and worst cases on average is less than a factor of two. In most cases, Options $1 \mathrm{a}$ and $2 \mathrm{a}-$ the "atrium" options - are most cost-effective. Unlike all the other options, which are compared with a conventional flat roof, these systems are compared with the specialized and costly construction of atria: they have the highest material credit and lowest additional construction costs, and no daylight or thermal benefits.

Options 1 and 2 are identical in construction and energy performance to Options $1 \mathrm{a}$ and $2 \mathrm{a}$, but are compared with the standard flat roof. They perform poorly, due to the high extra cost of skylight framing systems and low material credit 


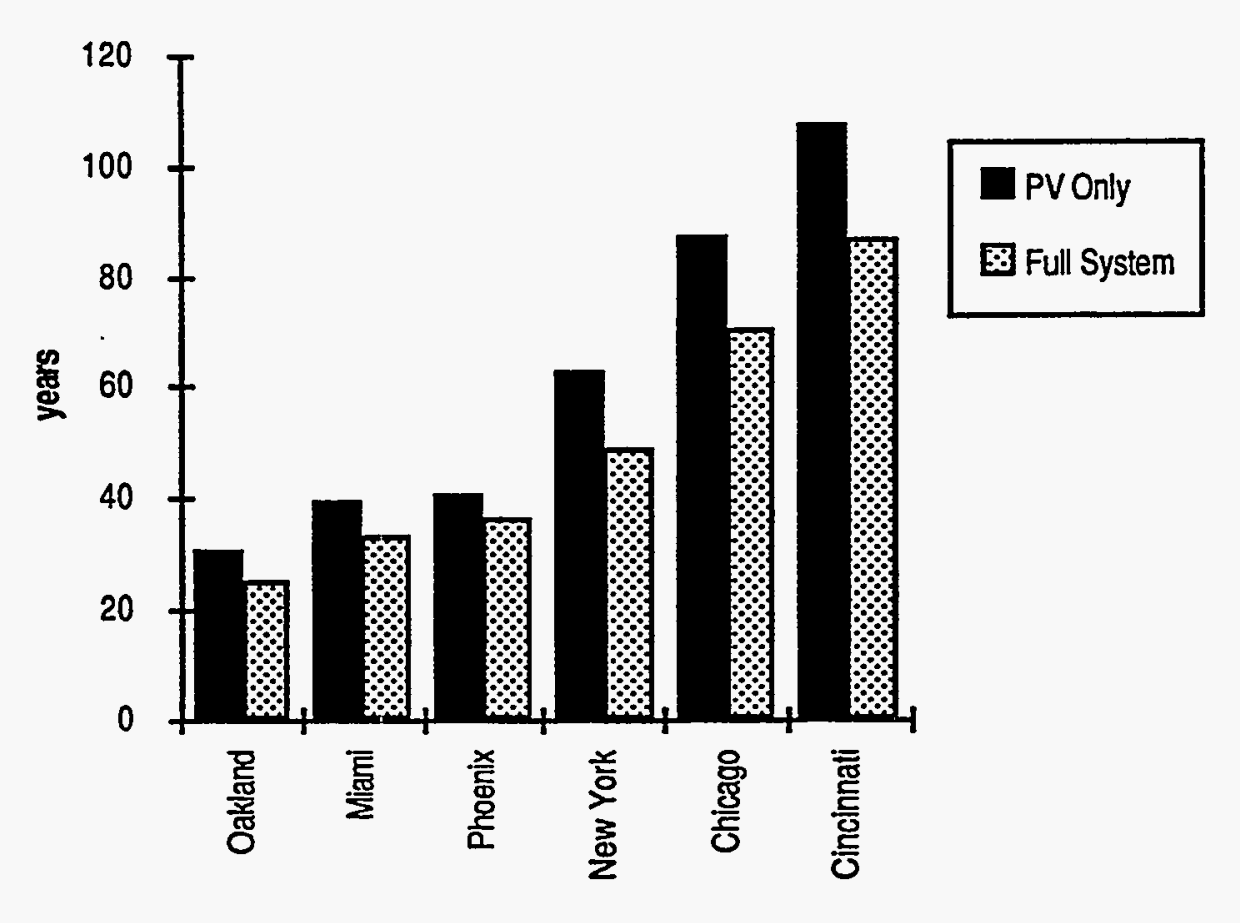

Chart 11. Average payback by option, all climates, all PV technologies.

for the conventional roof system and poor thermal performance. Option 1 is omitted from payback calculations because the very poor thermal performance results in extremely long or negative payback periods.

The roof options which rely most on collateral energy benefits (Options 4, 5, 5a), show the greatest improvement in payback when analyzed as a full system. We found that the active heat recovery of Option 5 is not generally cost-effective compared with the Option 4, which has the same indirect daylighting strategy but less expensive construction due to the absence of ductwork and a doublelayer skin. Since more heat is generated in all cases with Option 5 than is needed for building space heating, this system is inherently inefficient. Option 5a, which is identical to Option 5 except that it is assumed that all available heat is used, approaches Option 4 in payback time.

The ballasted roof-mounted system (Option 3) shows moderately long payback in all cases, since it has additional construction costs, no material credit and no significant collateral energy benefits.

The cost analyses reveal that many of the construction-related items cost as 


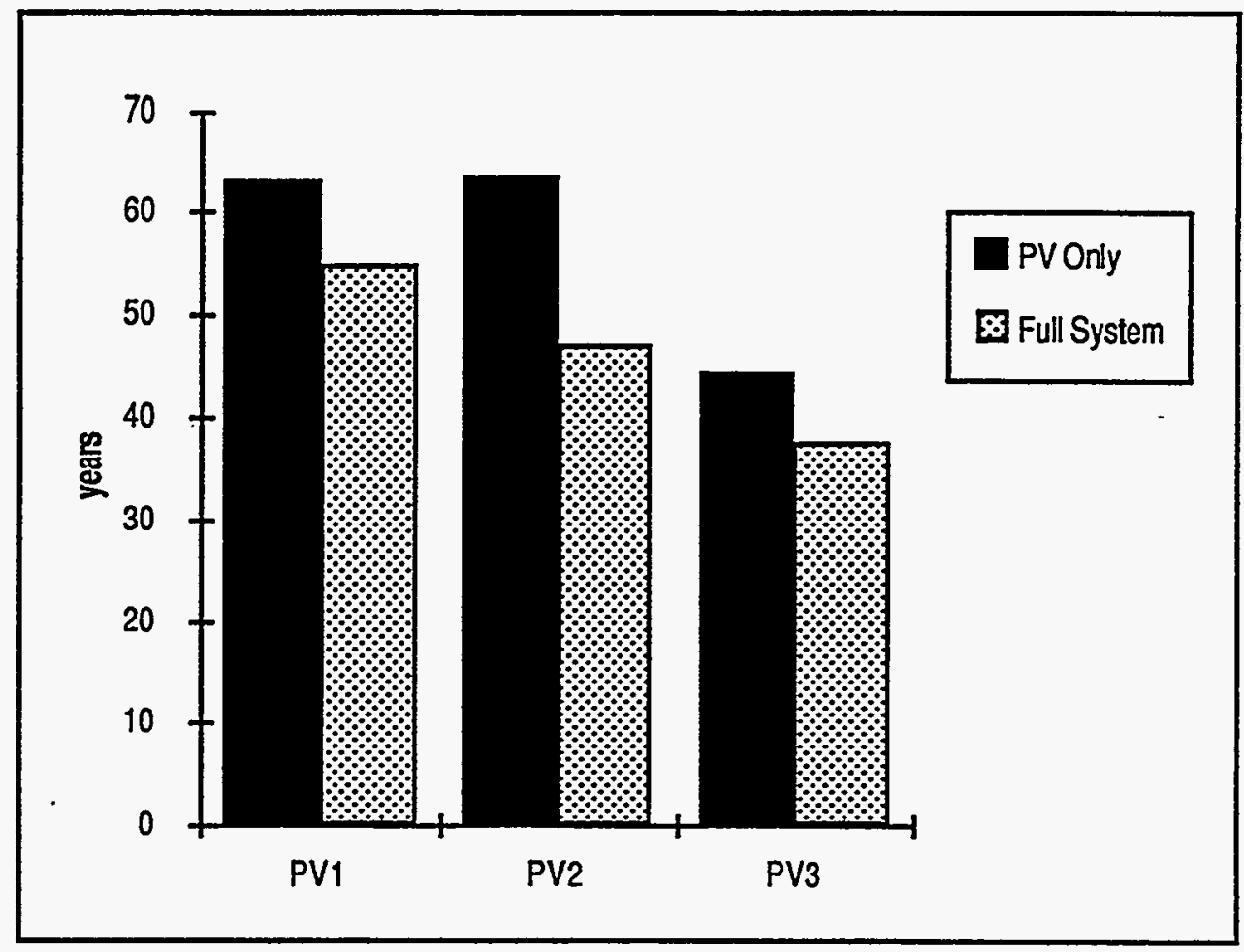

Chart 12. Average payback by PV technology, all locations, all options.

much or more than the PV modules. As PV costs decline, the cost/area of PVs becomes comparable to certain construction costs: PV1 is now $\$ 615 / \mathrm{m} 2$, PV3 is projected to be $\$ 215 / \mathrm{m} 2$. Skylight framing costs $\$ 215 / \mathrm{m} 2$, double glazing costs $\$ 231 / \mathrm{m} 2$. As PV costs drop, the costs of related systems become more significant, and the value of material credits becomes greater. In the future, the reduction of PV-related construction costs will be increasingly important.

\section{PV TECHNOLOGY.}

The three PV technologies evaluated are most cost-effective in very specific conditions. We have identified the three technologies as Crystalline silicon (PV1), amorphous silicon (PV2) and advanced thin-film, CIS or CdTe (PV3). The analysis is primarily on the basis of their cost and efficiency, although the effects of operating temperature on efficiency is taken into account for each material. Other properties of these devices - aesthetics, long-term stability, toxic materials or heavy metals - may significantly affect their viability as building materials, but are not considered in this report.

In all cases evaluated, PV3 systems have the shortest payback. Since PV3 materials are not presently available (although they may be within the next few 


\begin{tabular}{|c|c|c|c|c|c|c|c|}
\hline $\begin{array}{l}2 \\
3 \\
4 \\
5\end{array}$ & $\begin{array}{l}\text { Option2 } \\
\text { PV module } \\
\text { Area-independent costs } \\
\text { Area-dependent costs } \\
\text { Material Credit } \\
\text { Total with markuo \& location factor }\end{array}$ & \begin{tabular}{|l|} 
PV1 \\
$\$ / \mathrm{m} 2$ \\
$\$ 615.72$ \\
$\$ 167.92$ \\
$\$ 443.92$ \\
$(\$ 63.94)$ \\
$\$ 1.533 .98$ \\
\end{tabular} & $\begin{array}{r}\$ / W p \\
\$ 4.40 \\
\$ 1.20 \\
\$ 3.17 \\
(\$ 0.46) \\
\$ 10.96\end{array}$ & \begin{tabular}{|l|} 
PV2 \\
$\$ / \mathrm{m} 2$ \\
$\$ 155.01$ \\
$\$ 62.00$ \\
$\$ 443.92$ \\
$(\$ 63.94)$ \\
$\$ 793.09$ \\
\end{tabular} & $\begin{array}{l}\$ / W p \\
\$ 3.00 \\
\$ 1.20 \\
\$ 8.59 \\
(\$ 1.24) \\
\$ 15.35\end{array}$ & \begin{tabular}{|l|}
$\mathrm{PV} 3$ \\
\$/m2 \\
$\$ 215.29$ \\
$\$ 129.17$ \\
$\$ 443.92$ \\
$(\$ 63.94)$ \\
$\$ 959.73$ \\
\end{tabular} & $\begin{array}{l}\$ / W p \\
\$ 2.00 \\
\$ 1.20 \\
\$ 4.12 \\
(\$ 0.59) \\
\$ 8.92\end{array}$ \\
\hline 5 & Total with markup \& location factor & $\$ 1,533.98$ & $\$ 10.96$ & $\$ 793.09$ & $\$ 15.35$ & $\$ 959.73$ & $\$ 8.92$ \\
\hline $\begin{array}{l}6 \\
7\end{array}$ & $\begin{array}{l}\text { Value of PV output } \\
\text { Value of davliahththermal benefils }\end{array}$ & $\begin{array}{r}\$ 35.14 \\
\$ 8.33\end{array}$ & $\begin{array}{l}\$ 0.25 \\
\$ 0.06\end{array}$ & $\begin{array}{r}\$ 14.20 \\
\$ 8.33\end{array}$ & $\begin{array}{l}\$ 0.27 \\
\$ 0.16\end{array}$ & $\begin{array}{r}\$ 27.17 \\
\$ 8.33\end{array}$ & $\begin{array}{l}\$ 0.25 \\
\$ 0.08\end{array}$ \\
\hline 8 & Total beneffit & $\$ 43.47$ & $\$ 0.31$ & $\$ 22.54$ & $\$ 0.44$ & $\$ 35.50$ & $\$ 0.33$ \\
\hline 9 & Simple Payback & 35.29 & & 35.19 & & 27.03 & \\
\hline & Option2a & $|p|$ & & & & 13 & \\
\hline 10 & PV module & $\$ 615.72$ & $\underset{\$ 4.40}{\$ i W p}$ & $\$ 155.01$ & $\begin{array}{l}\$ / W p \\
\$ 3.00\end{array}$ & $\$$ & $\$ \$ \$ W p$ \\
\hline 11 & Area-independent costs & $\$ 167.92$ & $\$ 1.20$ & $\$ 62.00$ & $\$ 1.20$ & $\$ 129.16$ & $\$ 1.20$ \\
\hline 12 & Area-dependent costs & $\$ 228.63$ & $\$ 1.63$ & $\$ 228.63$ & $\$ 4.42$ & $\$ 228.63$ & $\$ 2.12$ \\
\hline 13 & Material Credit & $(\$ 231.43)$ & $(\$ 1.65)$ & (\$231.43) & $(\$ 4.48)$ & $(\$ 231.43\rangle$ & $(\$ 2.15)$ \\
\hline 14 & Total with markup \& location factor & $\$ 1,020.99$ & $\$ 7.30$ & $\$ 280.09$ & $\$ 5.42$ & $\$ 446.73$ & $\$ 4.15$ \\
\hline 15 & $\begin{array}{l}\text { Value of PV output } \\
\text { Value }\end{array}$ & $\$ 35.14$ & $\$ 0.25$ & $\$ 14.20$ & $\$ 0.27$ & $\$ 27.17$ & $\$ 0.25$ \\
\hline $\begin{array}{l}16 \\
17\end{array}$ & $\begin{array}{l}\text { Value of daylighth } \\
\text { Total benefit }\end{array}$ & $\frac{\$ 0.00}{\$ 35.14}$ & $\$ \$ \$ 0.00$ & $\frac{\$ 0.00}{\$ 14.21}$ & $\$ \$ \$ 0.00$ & $\begin{array}{r}\$ 0.00 \\
\$ 27.17\end{array}$ & $\$ 0.25$ \\
\hline 18 & Simple Payback & 29.05 & & 19.72 & & 16.44 & \\
\hline
\end{tabular}

Table 12. Area-dependent and Area-independent cost and income components. Sample for options 2, $2 a$, Oakland location factors.

years), an interesting comparison can be made between PV1 and PV2.

In general, PV1, high-cost, high-efficiency modules are best suited to installations where the rest of the area-dependent balance of system costs are high and area-dependent benefits are low. Table 12 uses the examples of options 2 and $2 a$ to illustrate the relative value of area-dependent and area-independent costs to different PV technologies. The area-dependent costs (framing, insulated glazing unit fabrication, wiring) are worth $\$ 3.17 / \mathrm{W}$ for PV1, and $\$ 8.59 / \mathrm{W}$ for PV2, well over twice as much (lines 3 and 12). Conversely, the area-dependent income (credit for displaced construction materials and daylight/thermal benefits) are worth proportionally more for PV2 than PV1 (lines 4, 7, 13, 16).

Ulimately, the best payback times are achieved with the lowest cost/watt modules, although there are many situations where area-dependent balance of system costs will make high-cost/high-efficiency modules worthwhile. Buildingintegrated applications tend to favor the former, while standalone systems can justify the latter. 


\section{PAYBACK ANALYSIS - CHARTS}

The following six charts illustrate the payback periods from Tables 11 and 12, sorted by PV Technology. The data is presented in two ways: Full System (all costs and all types of energy benefits) and PV System Only (no construction costs for daylight \& thermal systems, and no daylight/thermal benefits for Options 4, 5, and 5a). 


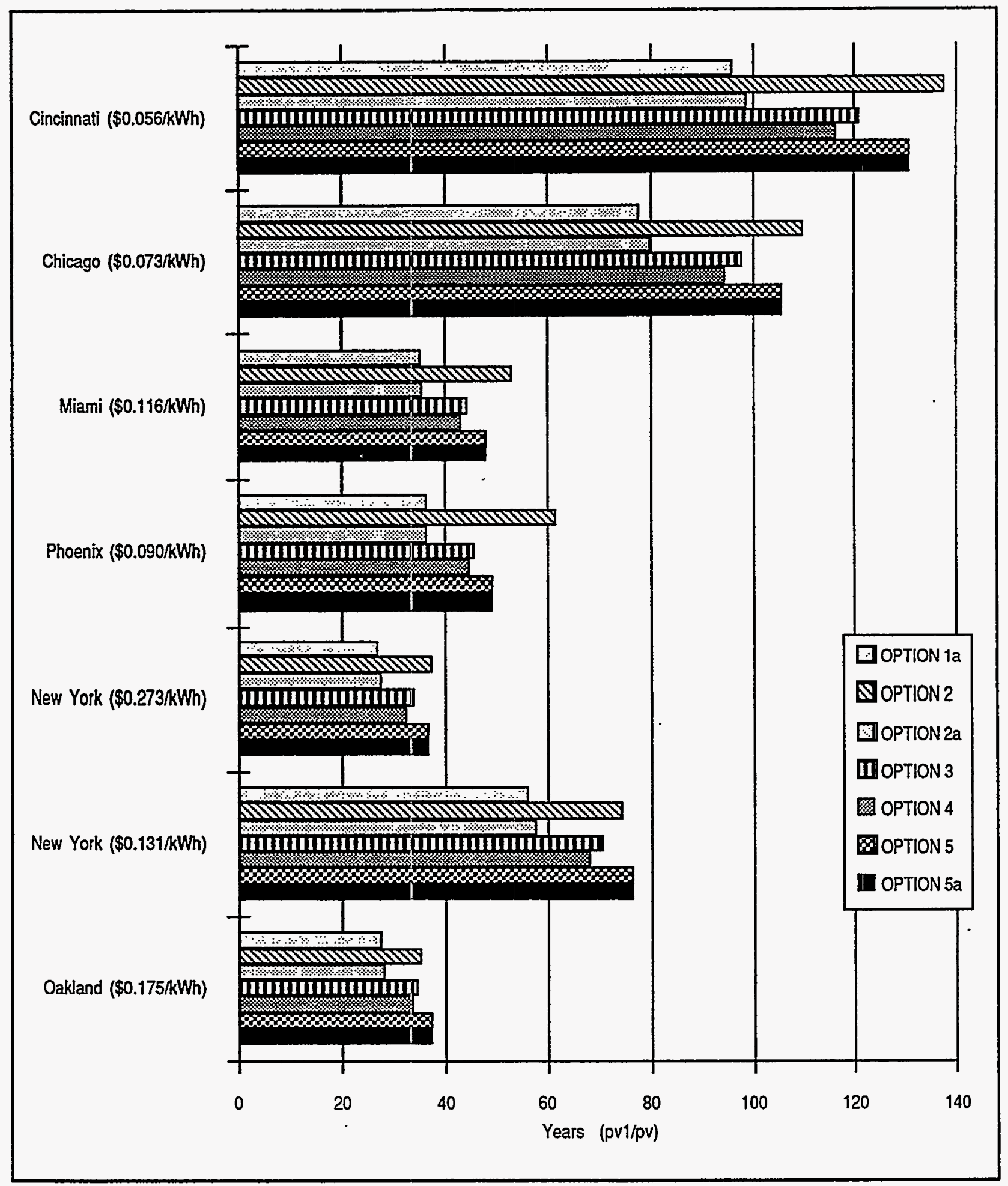

Chart 13. PV1 Payback, PV System Only (140W/m2, $\$ 4.40 / W)$. 


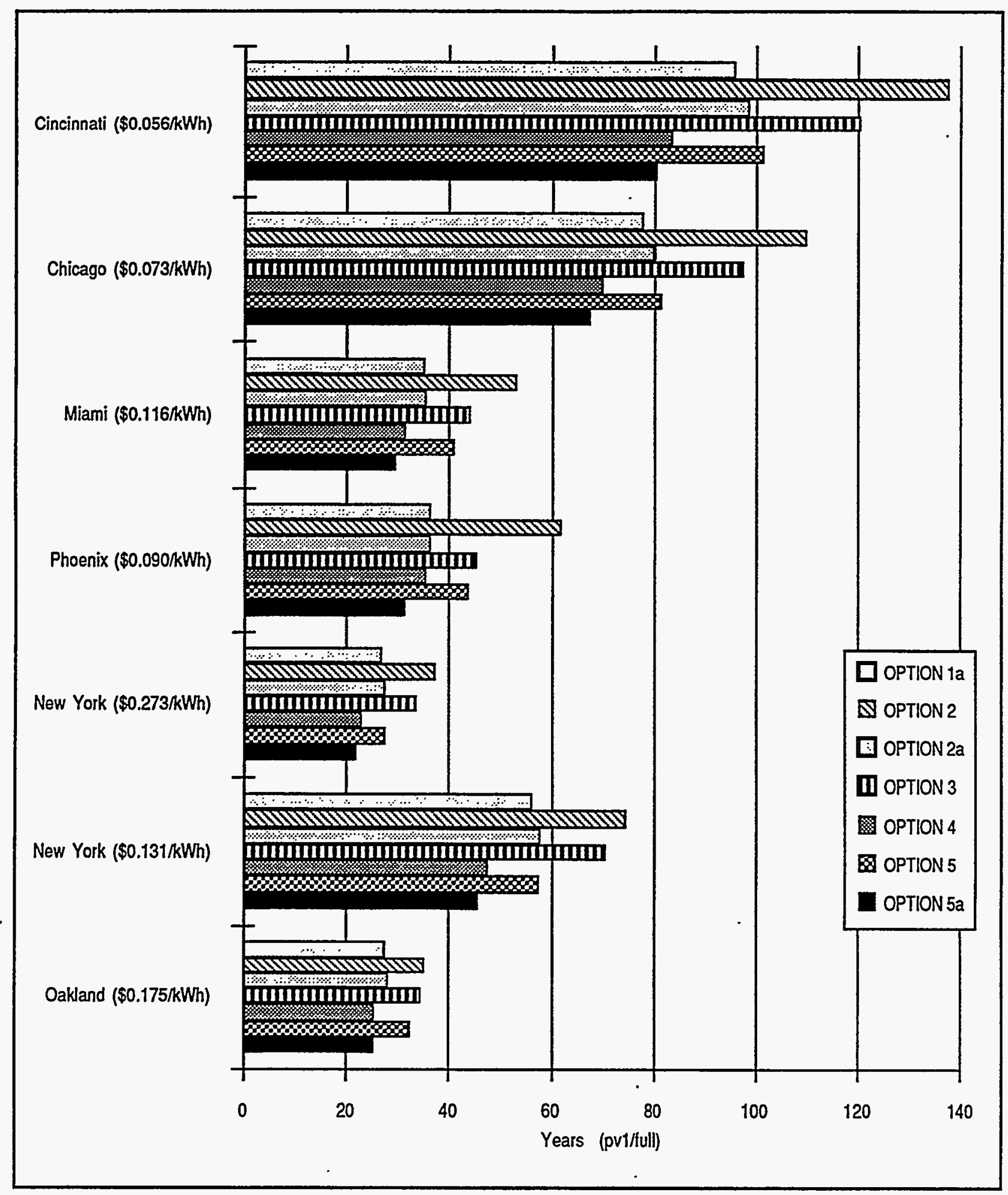

Chart 14. PV1 Payback, Full System (140W/m2, \$4.40/W). Includes daylight and thermal costs \& benefits. 


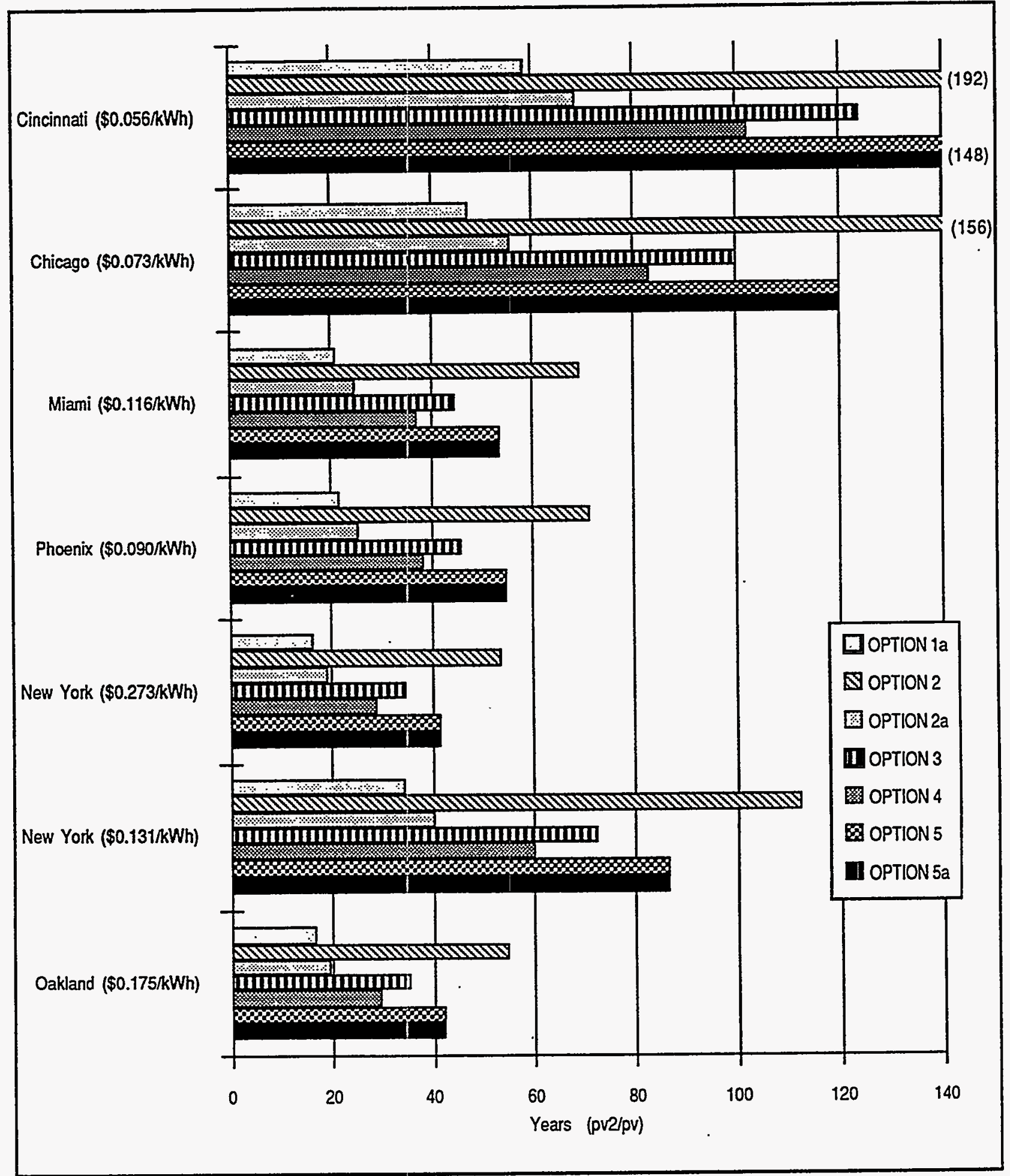

Chart 15. PV2 Payback, PV System Only (52W/m2, \$3.00/W). 


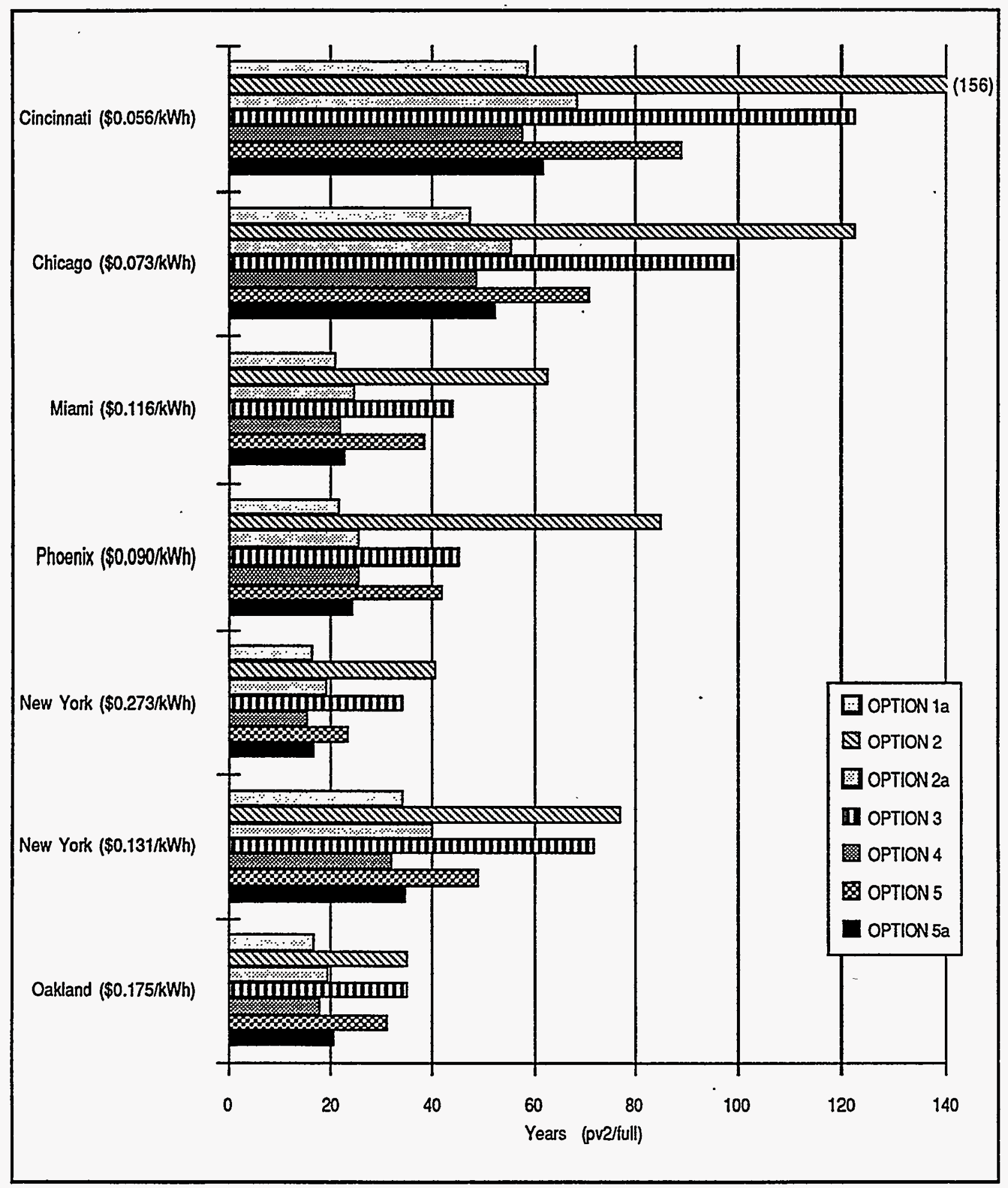

Chart 16. PV2 Payback, Full System $(52 W / m 2, \$ 3.00 / W)$. Includes daylight and thermal costs \& benefits. 


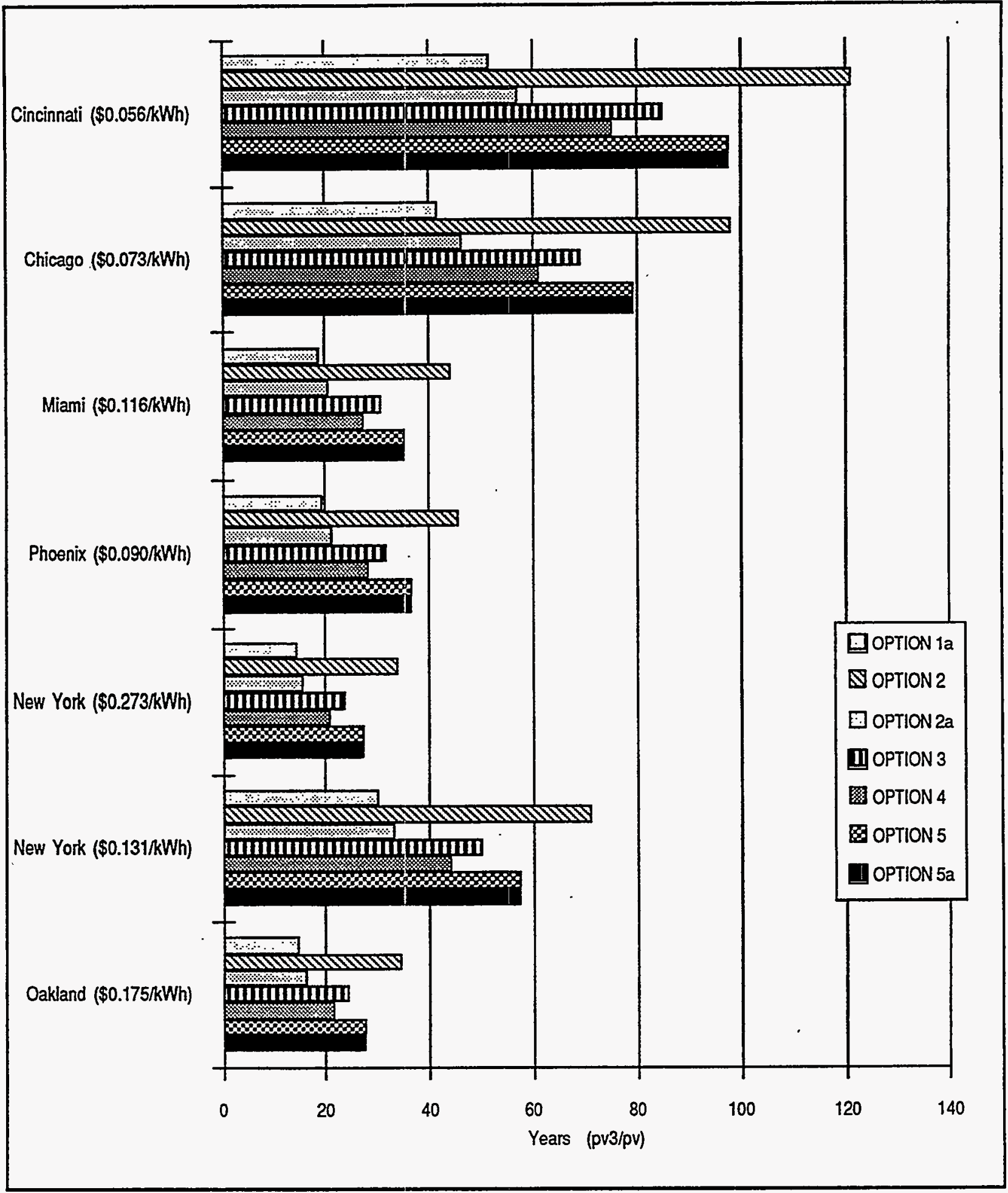

Chart 17. PV3 Payback, PV System Only (108W/m2, \$2.00/W). 


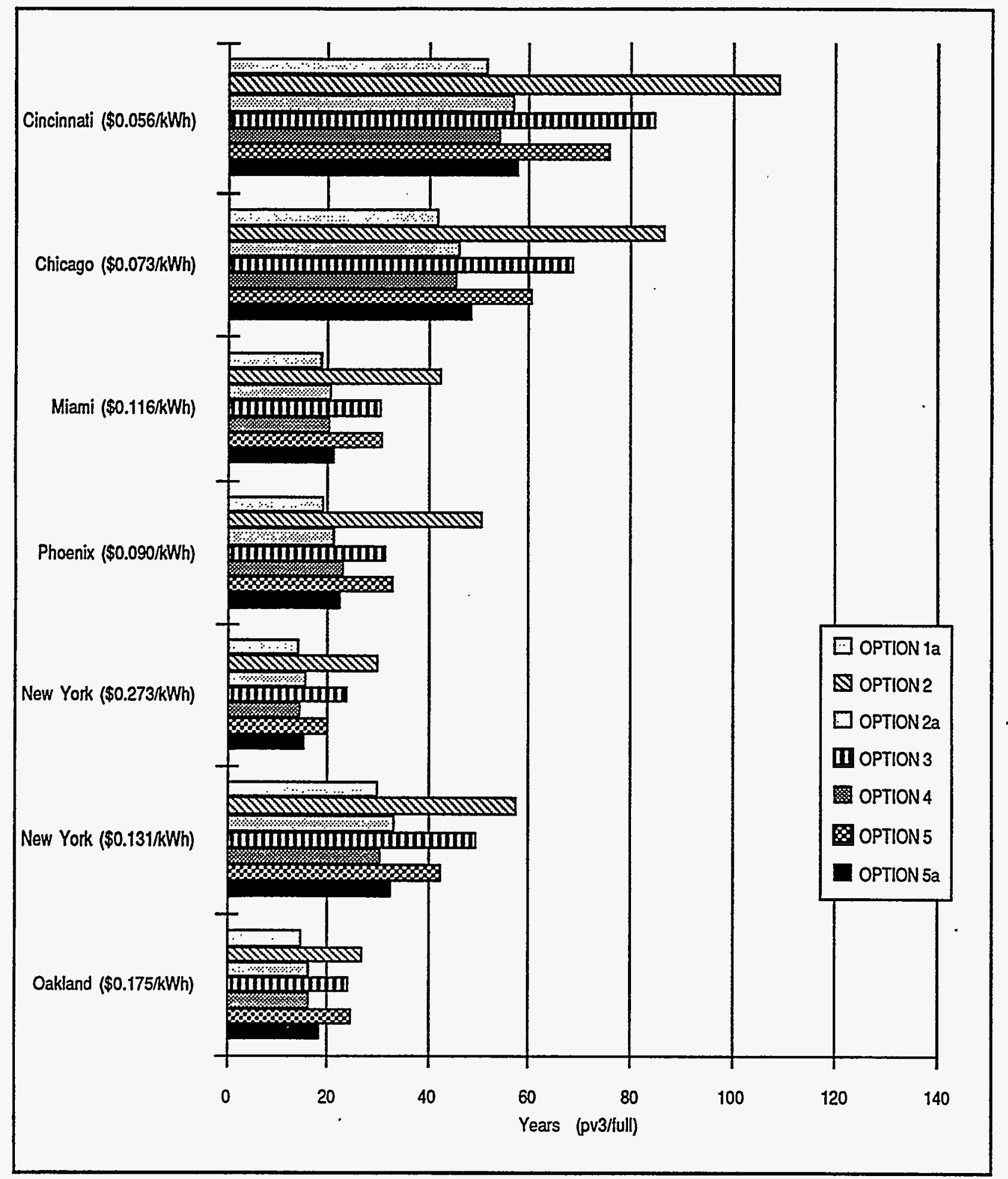

Chart 18. PV3 Payback, Full System (108W/m2, $\$ 2.00 / \mathrm{W})$. Includes daylight and thermal costs $\&$ benefits. 


\section{CONCLUSIONS:}

- Some BIPV roof systems are economical today. With present day technologies and utility rates, several of the systems analyzed show pre-tax payback of 20 years or less; with tax savings included these payback periods could be less than 10 years.

- There is no existing PV technology that is best suited for all BIPV roof applications. With current technologies and prices, low efficiency and low \$/m2 PVs like amorphous silicon show a better return in cases where area-dependent costs (skylight framing systems, insulated glazing unit fabrication) are low and/or area-dependent benefits (construction material credits, daylighting and thermal benefits) are high. Conversely, high efficiency and high \$/W PVs like crystalline silicon are better able to absorb area-dependent costs, and gain less by areadependent benefits.

- The best payback on a PV-only basis (ignoring collateral energy benefits) is from installations such as atriums with high material credit and low additional construction cost.

- Direct rooftop integration, where the PV module serves as the weather barrier for the roof, is not presently cost effective in cases other than atrium applications. The value of the material credit for conventional roofing materials is too low to offset the considerable costs of the framing systems required to accommodate large-area glass-substrate modules. Price reductions in these systems, or the development of other cost-competitive integrated roofing systems (such as PV metal roofing or PV tiles), could make direct integration viable in a much broader range of roof types.

- The value of PV can be significantly enhanced by collateral energy benefits. In most commercial buildings, these benefits will be mostly from daylighting thermal benefits are unlikely to be as great - for commercial buildings heating loads are a small portion of energy used. In cases where all the available thermal energy can be utilized, as in industrial processes with a large makeup air requirement, a very good payback can be achieved. 
- The viability of BIPV roof installations varies greatly with location. The combination of climate, insolation and electric rates in Oakland result in payback periods over three times as short as those in Cincinnati.

- Advances in PV technology will significantly improve the economics of BIPV systems. The better economics of lower cost/higher efficiency modules is clearly shown in the progression from PV1 to PV3. Most experts agree that costs and efficiencies better than PV3 eventually will be achieved.

- As the costs of PV technologies drop, the relative value of other construction systems (skylight framing, wiring) will increase. Any decrease in the costs of these systems will become more important to the cost effectiveness of the BIPV system as a whole. Conversely, the value of material credits for displaced construction materials will increase.

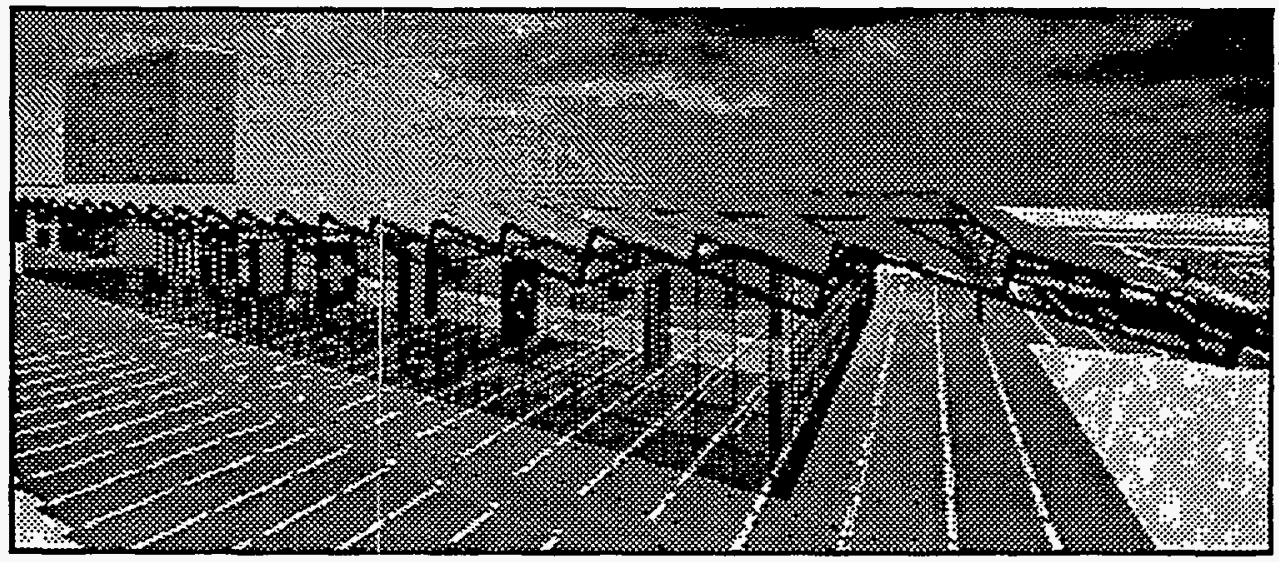

Fig 30. Conference center roof and shadow with hotel in background. 


\section{RECOMMENDATIONS FOR FUTURE STUDY}

\section{PRODUCT DEVELOPMENT:}

The construction cost estimates in this study are based on consultation with contractors, manufacturers, and on industry standard cost references. Since many of the BIPV products and applications evaluated have not been released as products, the cost estimates are preliminary. At present some of these products are being studied or developed; a compilation of ongoing development work would yield more reliable cost/performance data. Relevant research might include:

-Insulated PV glazing units

-Flush-glazed PV glazing units.

- Curtain wall extrusions with integral wiring raceways.

- Semitransparent PV window units in custom transparencies.

- Metal-substrate PV products for standing-seam roofing applications.

-Any development of products that will reduce the cost of PV-related construction elements (framing, wiring, etc.)

\section{RESEARCH:}

Other findings in this study should be investigated more thoroughly:

-The environmental analysis would benefit from detailed thermal evalua. tion of specific building designs including boundary conditions

-The payback analysis should include life cycle costs including O\&M costs, present value calculations, and other parameters.

- More research should be done on utility rate structures and the effects of the inclusion of environmental externalities and T\&D costs in electrical rates. 


\section{PRINCIPAL SOURCES}

Advanced Photovoltaic Systems, Inc., Princeton, NJ

Arthur D Little, Cambridge, MA

Ascension Technology, Inc., Lincoln Center, MA

Bell Products, Inc., Napa, CA

DeaMor, Inc., Vancouver, WA

Devcon Construction, Inc., Milpitas, CA

Energy Photovoltaics, Inc., Princeton, NJ

Gas Research Institute, Chicago, IL

New York Power Authority, NY, NY

Ove Arup \& Partners New York, NY

Ove Arup \& Partners San Francisco, CA

Pacific Gas and Electric, San Francisco, CA

Solar Design Associates, Harvard, MA

Viracon Corporation, Owatunna, MN

PV F-Chart Software, (1992 S.A. Klein and W. Beckman

RS Means Construction Cost Data 1994, RS Means Co., Inc. 


\section{APPENDICES}

Appendix A: Payback Analysis _- 69

Appendix B: Building Energy Balance Model: Parameters and Equations - 79 Appendix C: Building Energy Balance Calculations-_-85 Appendix D: PV Hourly Performance \& Utility Rate Calculations —_-123 


\section{A. PAYBACK ANALYSIS}

The following charts detail the payback calculations for all the roof options and climates analyzed. 


\begin{tabular}{|c|c|c|c|c|c|c|c|c|c|c|}
\hline & & $\begin{array}{l}\text { PV1: 140W/ms @ } \$ 4.40 / W \\
\text { Av } \$ / k W h \\
\text { Construction cost location fact. }\end{array}$ & $\begin{array}{r}\text { Oakkand } \\
\$ 0.175 \\
1.14 \\
\end{array}$ & $\begin{array}{r}\text { New York } 1 \\
\$ 0.131 \\
1.24 \\
\end{array}$ & $\begin{array}{c}\text { New York } 2 \\
\$ 0.273 \\
1.24 \\
\end{array}$ & $\begin{array}{c}\text { Phoenix } \\
\$ 0.090 \\
0.90\end{array}$ & $\begin{array}{c}\text { Mami } \\
\$ 0.116 \\
0.87\end{array}$ & $\begin{array}{c}\text { Chicago } \\
\$ 0.073 \\
1.06\end{array}$ & $\begin{array}{c}\text { Cincinnati } \\
\$ 0.056 \\
0.95 \\
\end{array}$ & Average \\
\hline \multirow[t]{17}{*}{ OPTION 1} & $\begin{array}{l}1 \\
2\end{array}$ & $\begin{array}{l}\text { Base system cost, } \$ / m 2 \\
\text { Additional HVAC, } \$ / m 2\end{array}$ & $\begin{array}{r}\$ 1,268.14 \\
\$ 77.64\end{array}$ & $\begin{array}{r}\$ 1,377.44 \\
\$ 113.00\end{array}$ & $\begin{array}{r}\$ 1,377.44 \\
\$ 113.00\end{array}$ & $\begin{array}{r}\$ 1,001.57 \\
\$ 142.49\end{array}$ & $\begin{array}{r}\$ 965.88 \\
\$ 99.18\end{array}$ & $\begin{array}{r}\$ 1,183.37 \\
\$ 103.13\end{array}$ & $\begin{array}{r}\$ 1,058.46 \\
\$ 95.10\end{array}$ & \\
\hline & 3 & Base system + HVAC & $\$ 1,345.78$ & $\$ 1,490.44$ & $\$ 1,490.44$ & $\$ 1,144.06$ & $\$ 1,065.07$ & $\$ 1,286.51$ & $\$ 1,153.56$ & \\
\hline & 5 & PV output & & & & & & & & \\
\hline & 6 & $\mathrm{kWh} / \mathrm{m} 2 / \mathrm{yr}$ & 202.72 & 145.17 & 145.17 & 236.79 & 182.37 & 161.81 & 152.22 & \\
\hline & 7 & $\$ / \mathrm{m} 2 / \mathrm{yr}$ & $\$ 35.54$ & $\$ 18.96$ & $\$ 39.59$ & $\$ 21.24$ & $\$ 21.12$ & $\$ 11.77$ & $\$ 8.53$ & \\
\hline & $\begin{array}{l}8 \\
9\end{array}$ & PVoniypayback,years & 35.68 & 72.65 & 34.79 & 47.15 & 45.74 & 100.54 & 124.02 & 65.80 \\
\hline & 10 & Lighting savings & & & & & & & & \\
\hline & 11 & $\mathrm{kWh} / \mathrm{m} 2 / \mathrm{yr}$ & 61.64 & 59.33 & 59.33 & 67.54 & 62.65 & 56.89 & 56.61 & \\
\hline & $\begin{array}{l}12 \\
13\end{array}$ & $\$ / m 2 / y r$ & $\$ 10.81$ & $\$ 7.75$ & $\$ 16.18$ & $\$ 6.06$ & $\$ 7.25$ & $\$ 4.14$ & $\$ 3.17$ & \\
\hline & 14 & Thermal savings (loss) & & & & & & & & \\
\hline & 15 & $\mathrm{kWh} / \mathrm{m} 2 / \mathrm{yr}$ & $(118.29)$ & $(165.92)$ & $(165.92)$ & $(334.16)$ & (246.37) & $(185.52)$ & $(200.35)$ & \\
\hline & 16 & $\$ / m 2 / y r$ & $(\$ 20.74)$ & $(\$ 21.67)$ & $(\$ 45.25)$ & $(\$ 29.97)$ & $(\$ 28.53)$ & $(\$ 13.49)$ & $\{\$ 11.23\}$ & \\
\hline & 17 & & & & & & & & & \\
\hline & 18 & PV+Lighting+Thermal & & & & & & & & \\
\hline & 19 & $\mathrm{kWh} / \mathrm{m} 2 / \mathrm{yr}$ & 146.07 & 38.58 & 38.58 & $(29.83)$ & (1.35) & 33.18 & 8.48 & \\
\hline & 20 & $\$ / \mathrm{m} 2 / \mathrm{yr}$ & $\$ 25.61$ & $\$ 5.04$ & $\$ 10.52$ & $(\$ 2.68)$ & $(\$ 0.16)$ & $\$ 2.41$ & $\$ 0.48$ & \\
\hline & 21 & Combinedpayback,years & 52.55 & 295.81 & 141.65 & $(427.53)$ & $(6,788.64)$ & 533.09 & $2,426.02$ & $(538.15)$ \\
\hline \multirow[t]{6}{*}{ OPTION 1a } & $\begin{array}{l}1 \\
2\end{array}$ & System cost, \$/m2 & $\$ 981.58$ & $\$ 1,066.18$ & $\$ 1,066.18$ & $\$ 775.25$ & $\$ 747.62$ & $\$ 915.97$ & $\$ 819.28$ & \\
\hline & 3 & PV output & & & & & & & & \\
\hline & 4 & $\mathrm{kWh} / \mathrm{m} 2 / \mathrm{yr}$ & 202.72 & 145.17 & 145.17 & 236.79 & 182.37 & 161.81 & 152.22 & \\
\hline & 5 & $\$ / m 2 / y r$ & $\$ 35.54$ & $\$ 18.96$ & $\$ 39.59$ & $\$ 21.24$ & $\$ 21.12$ & $\$ 11.77$ & $\$ 8.53$ & \\
\hline & 6 & Simplepayback,years & 27.62 & 56.24 & 26.93 & 36.50 & 35.41 & 77.82 & 96.00 & 50.93 \\
\hline & & & & $\cdot$ & & & & & & \\
\hline \multirow[t]{19}{*}{ OPTION 2} & 1 & System cost, $\$ / m 2$ & $\$ 1,521.49$ & $\$ 1,652.63$ & $\$ 1,652.63$ & $\$ 1,201.67$ & $\$ 1,158.85$ & $\$ 1,419.79$ & $\$ 1,269.91$ & \\
\hline & 2 & Additional HVAC, $\$ / m 2$ & $\$ 12.49$ & $\$ 22.79$ & $\$ 113.00$ & $\$ 35.78$ & $\$ 22.19$ & $\$ 20.79$ & $\$ 20.02$ & \\
\hline & $\begin{array}{l}3 \\
4\end{array}$ & Base system + HVAC & $\$ 1,533.98$ & $\$ 1,675.42$ & $\$ 1,765.62$ & $\$ 1,237.45$ & $\$ 1,181.03$ & $\$ 1,440.57$ & $\$ 1,289.94$ & \\
\hline & 5 & PV output & & & & & & & & \\
\hline & 6 & $\mathrm{kWh} / \mathrm{m} 2 / \mathrm{yr}$ & 200.44 & 143.81 & 143.81 & 233.80 & 180.28 & 160.20 & 150.75 & \\
\hline & 7 & $\$ / \mathrm{m} 2 / \mathrm{yr}$ & $\$ 35.14$ & $\$ 18.78$ & $\$ 39.22$ & $\$ 20.97$ & $\$ 20.87$ & $\$ 11.65$ & $\$ 8.45$ & \\
\hline & $\begin{array}{l}8 \\
9\end{array}$ & PVonly payback,years & 43.30 & 87.99 & 42.14 & 57.30 & 55.52 & 121.84 & 150.26 & 79.76 \\
\hline & 10 & Lighting savings & & & & & & & & \\
\hline & 11 & $\mathrm{kWh} / \mathrm{m} 2 / \mathrm{yr}$ & 61.64 & 59.33 & 59.33 & 67.54 & 62.65 & 56.89 & 56.61 & \\
\hline & 12 & $\$ / \mathrm{m} 2 / \mathrm{yr}$ & $\$ 10.81$ & $\$ 7.75$ & $\$ 16.18$ & $\$ 6.06$ & $\$ 7.25$ & $\$ 4.14$ & $\$ 3.17$ & \\
\hline & 13 & & & & & & & & & \\
\hline & 14 & Thermal savings (loss) & & & & & & & & \\
\hline & 15 & $\mathrm{kWh} / \mathrm{m} 2 / \mathrm{yr}$ & (14.12) & $(30.78)$ & $(30.78)$ & $(78.51)$ & $(51.80)$ & $(37.25)$ & $(40.24)$ & \\
\hline & 16 & $\$ / m 2 / y r$ & $(\$ 2.48)$ & $(\$ 4.02)$ & $(\$ 8.39)$ & $(\$ 7.04)$ & $(\$ 6.00)$ & $(\$ 2.71)$ & $(\$ 2.26)$ & \\
\hline & 17 & & & & & & & & & \\
\hline & 18 & PV+Lighting+Thermal & & & & & & & & \\
\hline & 19 & $\mathrm{kWh} / \mathrm{m} 2 / \mathrm{yr}$ & 247.96 & 172.36 & 172.36 & 222.83 & 191.13 & 179.84 & 167.12 & \\
\hline & 20 & $\$ / \mathrm{m} 2 / \mathrm{yr}$ & $\$ 43.47$ & $\$ 22.51$ & $\$ 47.01$ & $\$ 19.99$ & $\$ 22.13$ & $\$ 13.08$ & $\$ 9.37$ & \\
\hline & 21 & Combinedpayback,years & 35.29 & 74.43 & 37.56 & 61.91 & 53.37 & 110.12 & 137.67 & 72.91 \\
\hline \multirow[t]{6}{*}{ OPTION $2 a$} & $\begin{array}{l}1 \\
2\end{array}$ & System cost, $\$ / m 2$ & $\$ 1,020.99$ & $\$ 1,108.99$ & $\$ 1,108.99$ & $\$ 806.37$ & $\$ 777.64$ & $\$ 952.74$ & $\$ 852.17$ & \\
\hline & 3 & PV output & & & & & & & & \\
\hline & 4 & $\mathrm{kWh} / \mathrm{m} 2 / \mathrm{yr}$ & 200.44 & 143.81 & 143.81 & 233.80 & 180.28 & 160.20 & 150.75 & \\
\hline & 5 & $\$ / m 2 / y r$ & $\$ 35.14$ & $\$ 18.78$ & $\$ 39.22$ & $\$ 20.97$ & $\$ 20.87$ & $\$ 11.65$ & $\$ 8.45$ & \\
\hline & 6 & Simplepayback,years & 29.05 & 59.05 & 28.27 & 38.45 & 37.25 & 81.76 & 100.83 & 53.52 \\
\hline & \multicolumn{4}{|c|}{ Building-Integrated Photovoltaics: A Case Study } & Kiss and & dCompany Ar & rehitects & 211099 & 70 & \\
\hline
\end{tabular}


\begin{tabular}{lccccccc} 
PV1: 140W/ms @ \$4.40NW & Oakland & New York1 New York2 & Phoenix & Mami & Chicago & Cincinnati Average \\
Av \$/kWh & $\$ 0.175$ & $\$ 0.131$ & $\$ 0.273$ & $\$ 0.090$ & $\$ 0.116$ & $\$ 0.073$ & $\$ 0.056$ \\
Constuction cost location fact & 1.14 & 1.24 & 1.24 & 0.90 & 0.87 & 1.06 & 0.95 \\
\hline
\end{tabular}

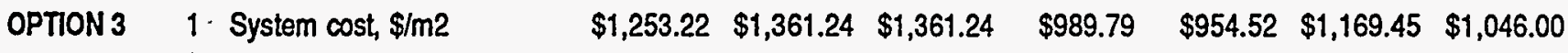

2

3

4 PV output

$5 \mathrm{kWh} / \mathrm{m} 2 / \mathrm{yr}$

$6 \$ / m 2 / y r$

PVonly payback, years

8

10 Lighting savings

$11 \mathrm{kWh} / \mathrm{m} 2 / \mathrm{yr}$

$12 \$ / \mathrm{m} 2 / \mathrm{yr}$

13

14

4
Thermal savings (loss)

$\mathrm{kWh} / \mathrm{m} 2 / \mathrm{yr}$

$\$ / \mathrm{m} 2 / \mathrm{yr}$

PV+Lighting+Thermal

$\mathrm{kWh} / \mathrm{m} 2 / \mathrm{yr}^{\circ}$

$\$ / \mathrm{m} 2 / \mathrm{yr}$

Combinedpayback,years

System cost, $\$ / m 2$

Additional HVAC, \$/m2

Base system + HVAC

System cost, PV only, \$/m2

PV output

$\mathrm{kWh} / \mathrm{m} 2 / \mathrm{yr}$

$\$ / m 2 / y r$

PVonlypayback,years

Lighting savings

$\mathrm{kWh} / \mathrm{m} 2 / \mathrm{yr}$

$\$ / \mathrm{m} 2 / \mathrm{yr}$

Thermal savings (loss)

$\mathrm{kWh} / \mathrm{m} 2 / \mathrm{yr}$

$\$ / m 2 / y r$

PV+Lighting+Thermal

$\mathrm{kWh} / \mathrm{m} 2 / \mathrm{yr}$

$\$ / \mathrm{m} 2 / \mathrm{yr}$

Combinedpayback,years

Lighting+Thermal

Lighitng/thermal payback, yrs

\begin{tabular}{rrrrrrr}
205.74 & 146.97 & 146.97 & 240.76 & 185.13 & 163.94 & 154.18 \\
$\$ 36.07$ & $\$ 19.19$ & $\$ 40.08$ & $\$ 21.60$ & $\$ 21.44$ & $\$ 11.93$ & $\$ 8.64$ \\
\hline $\mathbf{3 4 . 7 4}$ & $\mathbf{7 0 . 9 2}$ & $\mathbf{3 3 . 9 6}$ & $\mathbf{4 5 . 8 3}$ & $\mathbf{4 4 . 5 3}$ & $\mathbf{9 8 . 0 7}$ & $\mathbf{1 2 1 . 0 1}$
\end{tabular}

64.15

\begin{tabular}{|c|c|c|c|c|c|}
\hline 0.00 & 0.00 & 0.00 & 0.00 & 0.00 & 0.00 \\
\hline .00 & $\$ 0.00$ & $\$ 0.00$ & $\$ 0.00$ & $\$ 0.00$ & $\$ 0.00$ \\
\hline
\end{tabular}

\begin{tabular}{|c|c|c|c|c|c|}
\hline 0.45 & 0.53 & 0.53 & 1.21 & 0.97 & 0.55 \\
\hline$\$ 0.08$ & $\$ 0.07$ & $\$ 0.14$ & $\$ 0.11$ & $\$ 0.11$ & $\$ 0.04$ \\
\hline
\end{tabular}

\begin{tabular}{|c|c|c|c|c|c|c|}
\hline 206.19 & 147.50 & 147.50 & 241.97 & 186.10 & 164.49 & 154.83 \\
\hline$\$ 36.15$ & $\$ 19.26$ & $\$ 40.23$ & $\$ 21.70$ & $\$ 21.55$ & $\$ 11.97$ & $\$ 8.68$ \\
\hline 34.67 & 70.67 & 33.84 & 45.60 & 44.30 & 97.74 & 120.50 \\
\hline
\end{tabular}

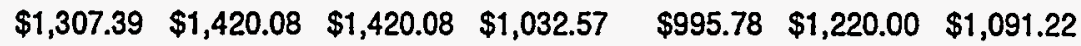

$\begin{array}{lllllll}(\$ 17.85) & (\$ 16.14) & (\$ 16.14) & (\$ 7.81) & (\$ 9.79) & (\$ 13.59) & (\$ 12.16)\end{array}$

\begin{tabular}{lllllll}
\hline$\$ 1,289.54$ & $\$ 1,403.94$ & $\$ 1,403.94$ & $\$ 1,024.77$ & $\$ 985.99$ & $\$ 1,206.41$ & $\$ 1,079.06$
\end{tabular}

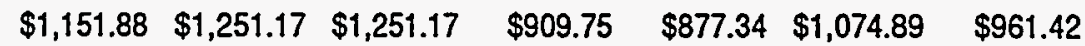

\begin{tabular}{|c|c|c|c|c|c|c|}
\hline 194.72 & 140.40 & 140.40 & 226.28 & 175.03 & 156.15 & 147.03 \\
\hline$\$ 34.14$ & $\$ 18.34$ & $\$ 38.29$ & $\$ 20.30$ & $\$ 20.27$ & $\$ 11.36$ & $\$ 8.24$ \\
\hline 33.74 & 68.24 & 32.68 & 44.82 & 43.29 & 94.63 & 116.63 \\
\hline
\end{tabular}

$\begin{array}{lllllll}61.64 & 59.33 & 59.33 & 67.54 & 62.65 & 56.89 & 56.61\end{array}$

$\begin{array}{lllllll}\$ 10.81 & \$ 7.75 & \$ 16.18 & \$ 6.06 & \$ 7.25 & \$ 4.14 & \$ 3.17\end{array}$

$\begin{array}{lllllll}33.54 & 25.65 & 25.65 & 27.84 & 30.59 & 24.38 & 26.06\end{array}$

$\begin{array}{lllllll}\$ 5.88 & \$ 3.35 & \$ 7.00 & \$ 2.50 & \cdot \$ 3.54 & \$ 1.77 & \$ 1.46\end{array}$

\begin{tabular}{|c|c|c|c|c|c|c|}
\hline 289.90 & 225.38 & 225.38 & 321.66 & 268.27 & 237.42 & 229.70 \\
\hline$\$ 50.83$ & $\$ 29.43$ & $\$ 61.47$ & $\$ 28.85$ & $\$ 31.06$ & $\$ 17.27$ & $\$ 12.88$ \\
\hline 25.37 & 47.70 & 22.84 & 35.52 & 31.74 & 69.86 & 83.79 \\
\hline
\end{tabular}

\begin{tabular}{rrrrrrrr}
$\$ 16.69$ & $\$ 11.10$ & $\$ 23.18$ & $\$ 8.56$ & $\$ 10.80$ & $\$ 5.91$ & $\$ 4.63$ & \\
\cline { 1 - 7 } & 13.10 & 6.43 & 13.54 & 10.19 & 22.11 & 25.51 & 14.19
\end{tabular}




\begin{tabular}{lrrrrrrr} 
PV1: 140W/ms @ \$4.40N & Oakland & \multicolumn{2}{c}{ New York1 New York2 } & Phoenix & Miami & Chicago & Cincinnati Average \\
Av \$/kWh & $\$ 0.175$ & $\$ 0.131$ & $\$ 0.273$ & $\$ 0.090$ & $\$ 0.116$ & $\$ 0.073$ & $\$ 0.056$ \\
Construction cost location fact & 1.14 & 1.24 & 1.24 & 0.90 & 0.87 & 1.06 & 0.95 \\
\hline
\end{tabular}

OPTION 5

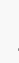

Additional HVAC, $\$ / m 2$

Base system + HVAC

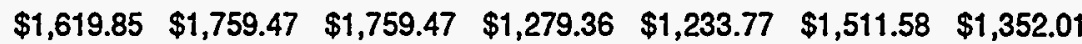

$\begin{array}{lllllll}(\$ 17.85) & (\$ 16.14) & (\$ 16.14) & (\$ 7.81) & (\$ 9.79) & (\$ 13.59) & (\$ 12.16)\end{array}$

$\begin{array}{lllllll}\$ 1,602.00 & \$ 1,743.33 & \$ 1,743.33 & \$ 1,271.55 & \$ 1,223.98 & \$ 1,497.99 & \$ 1,339.86\end{array}$

System cost, PV only, \$/m2

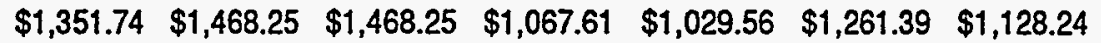

PV output

$\mathrm{kWh} / \mathrm{m} 2 / \mathrm{yr}$

$\$ / \mathrm{m} 2 / \mathrm{yr}$

PVonlypayback,years

Lighting savings

$\mathrm{kWh} / \mathrm{m} 2 / \mathrm{yr}$

$\$ / \mathrm{m} 2 / \mathrm{yr}$

Thermal savings (loss)

$\mathrm{kWh} / \mathrm{m} 2 / \mathrm{yr}$

$\$ / \mathrm{m} 2 / \mathrm{yr}$

PV+Lighting+Thermal

$\mathrm{kWh} / \mathrm{m} 2 / \mathrm{yr}$

$\$ / \mathrm{m} 2 / \mathrm{yr}$

Combinedpayback,years

Max thermal savings (loss)

$\mathrm{kWh} / \mathrm{m} 2 / \mathrm{yr}$

$\$ / \mathrm{m} 2 / \mathrm{yr}$

PV+Lighting+Max thermal

$\mathrm{kWh} / \mathrm{m} 2 / \mathrm{yr}$

$\$ / \mathrm{m} 2 / \mathrm{yr}$

Combined(maxthermal),yrs

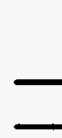

$\begin{array}{lllllll}344.22 & 280.54 & 280.54 & 431.84 & 341.22 & 294.80 & 286.24\end{array}$

$\begin{array}{lllllll}\$ 60.35 & \$ 36.64 & \$ 76.51 & \$ 38.74 & \$ 39.51 & \$ 21.44 & \$ 16.05\end{array}$

$\$ 7.58-\$ 7.51$

22.79

32.83

$\begin{array}{lrr}30.98 & 69.86 & 83.49\end{array}$

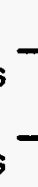

\begin{tabular}{rrrrrrr}
$\$ 11.39$ & $\$ 10.13$ & $\$ 21.16$ & $\$ 6.49$ & $\$ 7.25$ & $\$ 5.84$ & $\$ 4.10$ \\
\hline 21.75 & 26.78 & 12.82 & 31.56 & 26.98 & 40.40 & 51.79 \\
$\$ 24.42$ & $\$ 17.50$ & $\$ 36.55$ & $\$ 17.23$ & $\$ 18.16$ & $\$ 9.56$ & $\$ 7.43$ \\
\hline 10.15 & 15.50 & 7.42 & 11.88 & 10.78 & 24.66 & 28.56
\end{tabular}

44.87

30.30

15.56 


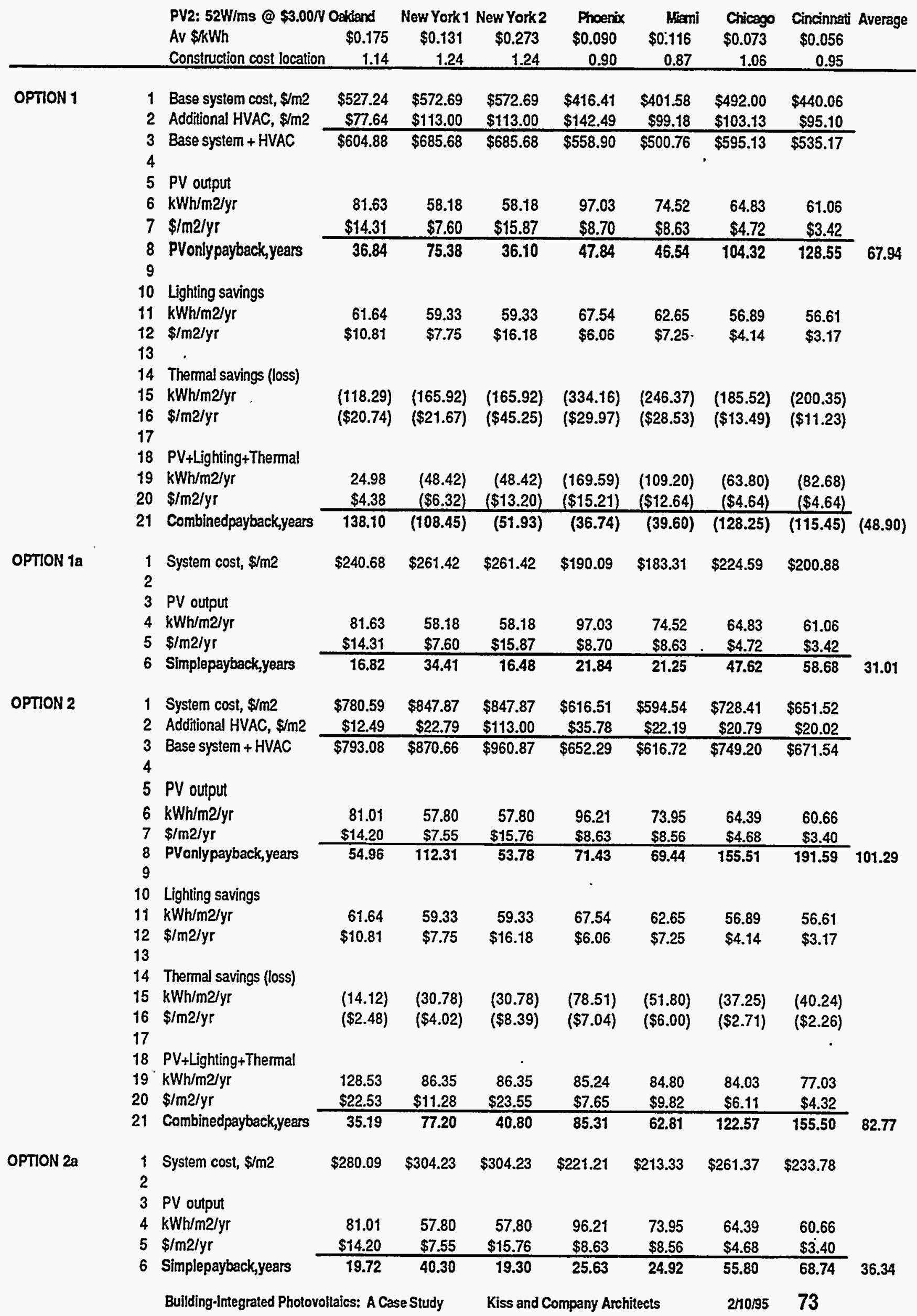


PV1: 140W/ms @ \$4.40, Oakdand New York1 New York2 Phoenix Mixi Chicago Cincinnati Average $\begin{array}{llllllll}\text { Av } \$ / k W h & \$ 0.175 & \$ 0.131 & \$ 0.273 & \$ 0.090 & \$ 0.116 & \$ 0.073 & \$ 0.056\end{array}$

$\begin{array}{lllllllll}\text { Construction cost location } & 1.14 & 1.24 & 1.24 & 0.90 & 0.87 & 1.06 & 0.95\end{array}$

OPTION 3

System cost, $\$ / \mathrm{m} 2$

$\$ 512.3$

2

3

4 PV output

$5 \mathrm{kWh} / \mathrm{m} 2 / \mathrm{yr}$

$\$ / \mathrm{m} 2 / \mathrm{yr}$

PVonlypayback,years

10 Lighting savings

$11 \mathrm{kWh} / \mathrm{m} 2 / \mathrm{yr}$

$12 \$ / \mathrm{m} 2 / \mathrm{yr}$

13

14

15

16

17

$18 \mathrm{PV}+$ Lighting+Thermal

$19 \mathrm{kWh} / \mathrm{m} 2 / \mathrm{yr}$

$20 \$ / \mathrm{m} 2 / \mathrm{yr}$

21 Combinedpayback,years

Thermal savings (loss)

$\mathrm{kWh} / \mathrm{m} 2 / \mathrm{yr}$

$\$ / m 2 / y r$

\begin{tabular}{rrr}
82.46 & 58.67 & 58.67 \\
$\$ 14.46$ & $\$ 7.66$ & $\$ 16.00$ \\
\hline 35.44 & 72.63 & 34
\end{tabular}

58.67

$\$ 16.00$

34.78

0.00

$\$ 0.00$

$$
0.00
$$

$\$ 0.00$

0.00
$\$ 0.00$

0.00

0.00

0.00

$\$ 0.00$

0.00

0.45

$\$ 0.08$

0.53

$\$ 0.07$

0.53
$\$ 0.14$

1.21

$\begin{array}{lllll}\$ 0.14 & \$ 0.11 & \$ 0.11 & \$ 0.04 & \$ 0.04\end{array}$

0.97

0.55

$\begin{array}{lllll}\$ 0.14 & \$ 0.11 & \$ 0.11 & \$ 0.04 & \$ 0.04\end{array}$

0.65

OPTION 4

\begin{tabular}{|c|c|c|c|c|c|c|}
\hline 82.91 & 59.20 & 59.20 & 99.33 & 76.25 & 65.97 & 62.25 \\
\hline$\$ 14.54$ & $\$ 7.73$ & $\$ 16.14$ & $\$ 8.91$ & $\$ 8.83$ & $\$ 4.80$ & $\$ 3.49$ \\
\hline 35.25 & 71.98 & 34.47 & 45.41 & 44.20 & 99.63 & 122.53 \\
\hline
\end{tabular}

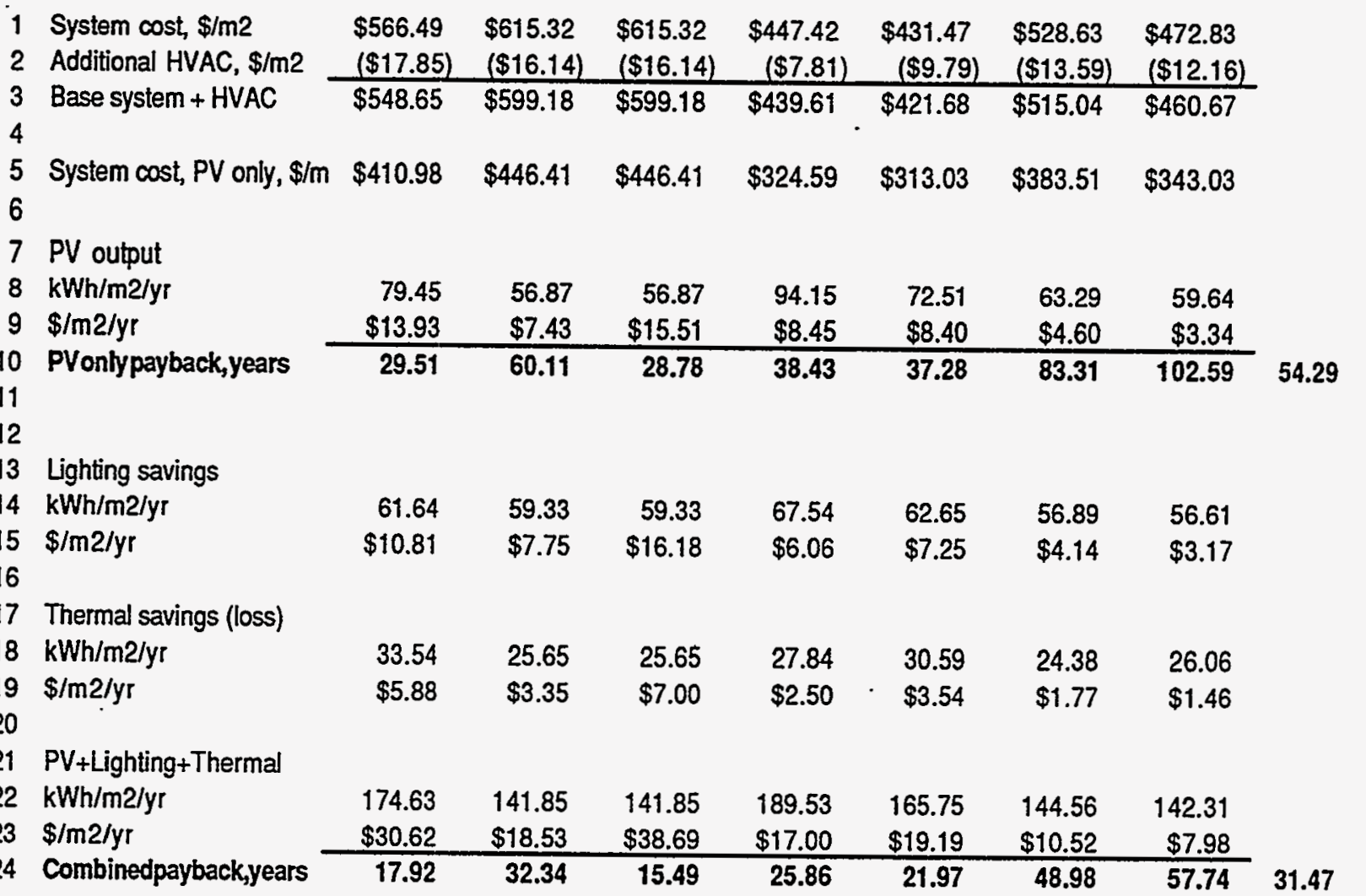


PV1: 140W/ms @ \$4.40, Oakland New York1 New York2 Phoenix Miami Chicago Cincinnati Average $\begin{array}{llllllll}\text { Av } \$ k W h & \$ 0.175 & \$ 0.131 & \$ 0.273 & \$ 0.090 & \$ 0.116 & \$ 0.073 & \$ 0.056\end{array}$

$\begin{array}{lllllllll}\text { Construction cost location } & 1.14 & 1.24 & 1.24 & 0.90 & 0.87 & 1.06 & 0.95\end{array}$

\begin{tabular}{|c|c|c|c|c|c|c|c|c|}
\hline 1 System cost, \$/m2 & $\begin{array}{l}\$ 878.95 \\
(\$ 1785)\end{array}$ & $\$ 954.71$ & $\$ 954.71$ & $\$ 694.20$ & $\$ 669.46$ & $\$ 820.20$ & $\$ 733.62$ & \\
\hline Additional HVAC, \$/m2 & & & & $(\$ 7.81)$ & $(\$ 9.79)$ & $(\$ 13.59)$ & & \\
\hline $\begin{array}{l}3 \text { Base system + HVAC } \\
4\end{array}$ & $\$ 861.11$ & $\$ 938.57$ & $\$ 938.57$ & $\$ 686.39$ & $\$ 659.67$ & $\$ 806.61$ & $\$ 721.47$ & \\
\hline $\begin{array}{l}5 \text { System cost, PV only, \$/m } \\
6\end{array}$ & $\$ 610.85$ & $\$ 663.50$ & $\$ 663.50$ & $\$ 482.45$ & $\$ 465.25$ & $\$ 570.02$ & $\$ 509.84$ & \\
\hline 7 PV output & & & & & & & & \\
\hline $\mathrm{kWh} / \mathrm{m} 2 / \mathrm{yr}$ & 82.25 & 58.54 & 58.54 & 97.84 & 75.08 & 65.27 & 61.46 & \\
\hline$\$ / m 2 / y r$ & $\$ 14.42$ & $\$ 7.65$ & $\$ 15.97$ & $\$ 8.78$ & $\$ 8.69$ & $\$ 4.75$ & $\$ 3.45$ & \\
\hline PVonlypayback,years & 42.36 & 86.78 & 41.56 & 54.97 & 53.52 & 120.06 & 147.97 & 78.17 \\
\hline \multicolumn{9}{|l|}{ Lighting savings } \\
\hline $\mathrm{kWh} / \mathrm{m} 2 / \mathrm{yr}$ & 61.64 & 59.33 & 59.33 & 67.54 & 62.65 & 56.89 & 56.61 & \\
\hline$\$ / \mathrm{m} 2 / \mathrm{yr}$ & $\$ 10.81$ & $\$ 7.75$ & $\$ 16.18$ & $\$ 6.06$ & $\$ 7.25$ & $\$ 4.14$ & $\$ 3.17$ & \\
\hline \multicolumn{9}{|l|}{ Thermal savings (loss) } \\
\hline $\mathrm{kWh} / \mathrm{m} 2 / \mathrm{yr}$ & 3.34 & 18.25 & 18.25 & 4.79 & 0.00 & 23.33 & 16.49 & \\
\hline$\$ / \mathrm{m} 2 / y \mathrm{r}$ & $\$ 0.59$ & $\$ 2.38$ & $\$ 4.98$ & $\$ 0.43$ & $\$ 0.00$ & $\$ 1.70$ & $\$ 0.92$ & \\
\hline \multicolumn{9}{|l|}{ PV+Lighting+Thermal } \\
\hline$k W h / m 2 / y r$ & 147.23 & 136.12 & 136.12 & 170.17 & 137.73 & 145.49 & 134.56 & \\
\hline$\$ / \mathrm{m} 2 / \mathrm{yr}$ & $\$ 25.81$ & $\$ 17.78$ & $\$ 37.12$ & $\$ 15.26$ & $\$ 15.95$ & $\$ 10.58$ & $\$ 7.54$ & \\
\hline Combinedpayback,years & 33.36 & 52.80 & 25.28 & 44.97 & 41.37 & 76.22 & 95.63 & 52.80 \\
\hline \multicolumn{9}{|l|}{ Max thermal savings (loss) } \\
\hline $\mathrm{kWh} / \mathrm{m} 2 / \mathrm{yr}$ & 77.62 & 74.70 & 74.70 & 124.56 & 94.15 & 74.52 & 75.95 & \\
\hline$\$ / \mathrm{m} 2 / \mathrm{yr}$ & $\$ 13.61$ & $\$ 9.76$ & $\$ 20.37$ & $\$ 11.17$ & $\$ 10.90$ & $\$ 5.42$ & $\$ 4.26$ & \\
\hline \multicolumn{9}{|l|}{ PV+Lighting+Max thermal } \\
\hline $\mathrm{kWh} / \mathrm{m} 2 / \mathrm{yr}$ & 221.51 & 192.57 & 192.57 & 289.94 & 231.88 & 196.68 & 194.02 & \\
\hline$\$ / \mathrm{m} 2 / \mathrm{yr}$ & $\$ 38.84$ & $\$ 25.15$ & $\$ 52.52$ & $\$ 26.01$ & $\$ 26.85$ & $\$ 14.31$ & $\$ 10.88$ & \\
\hline Combined(maxthermal),y & 22.17 & 37.32 & 17.87 & 26.39 & 24.57 & 56.38 & $\overline{66}$ & \\
\hline
\end{tabular}


PV3: 108W/ms @ \$2.00, Oakland New York1 New York2 Phoenix Miami Chicago Cincinnati Average $\begin{array}{llllllll}\text { Av } \$ / k_{W h}{ }^{*} & \$ 0.175 & \$ 0.131 & \$ 0.273 & \$ 0.090 & \$ 0.116 & \$ 0.073 & \$ 0.056\end{array}$ \begin{tabular}{lllllllll} 
Construction cost location & 1.14 & 1.24 & 1.24 & 0.90 & 0.87 & 1.06 & 0.95 \\
\hline
\end{tabular}

\section{OPTION 1}

\begin{tabular}{lrrrrrrrr} 
Base system cost, $\$ / m 2$ & $\$ 693.89$ & $\$ 753.70$ & $\$ 753.70$ & $\$ 548.03$ & $\$ 528.50$ & $\$ 647.51$ & $\$ 579.16$ \\
2 Additional HVAC, $\$ / m 2$ & $\$ 77.64$ & $\$ 113.00$ & $\$ 113.00$ & $\$ 142.49$ & $\$ 99.18$ & $\$ 103.13$ & $\$ 95.10$ \\
\cline { 2 - 8 } & Base system + HVAC & $\$ 771.53$ & $\$ 866.69$ & $\$ 866.69$ & $\$ 690.52$ & $\$ 627.69$ & $\$ 750.64$ & $\$ 674.26$
\end{tabular}

5 PV output

$6 \mathrm{kWh} / \mathrm{m} 2 / \mathrm{yr}$

$7 \$ / \mathrm{m} 2 / \mathrm{yr}$

8 PVonlypayback,years

\begin{tabular}{rrrrrrr}
156.46 & 111.77 & 111.77 & 184.37 & 141.79 & 124.58 & 117.26 \\
$\$ 27.43$ & $\$ 14.60$ & $\$ 30.48$ & $\$ 16.54$ & $\$ 16.42$ & $\$ 9.06$ & $\$ 6.57$ \\
\hline 25.30 & $\mathbf{5 1 . 6 3}$ & $\mathbf{2 4 . 7 2}$ & 33.14 & $\mathbf{3 2 . 1 9}$ & $\mathbf{7 1 . 4 6}$ & $\mathbf{8 8 . 1 0}$
\end{tabular}

46.65

Lighting savings

$\mathrm{kWh} / \mathrm{m} 2 / \mathrm{yr}$

$\begin{array}{lllllll}61.64 & 59.33 & 59.33 & 67.54 & 62.65 & 56.89 & 56.61\end{array}$

$\$ / m 2 / y r$

$\$ 10.81$

$\$ 7.75 \quad \$ 16.18$

$\$ 6.06 \quad \$ 7.25$

$\$ 4.14$

$\$ 3.17$

14 Thermal savings (loss)

$15 \mathrm{kWh} / \mathrm{m} 2 / \mathrm{yr}$

$\begin{array}{lllllll}(118.29) & (165.92) & (165.92) & (334.16) & (246.37) & (185.52) & (200.35)\end{array}$

$\$ / \mathrm{m} 2 / \mathrm{yr}$

$(\$ 20.74) \quad(\$ 21.67) \quad(\$ 45.25)$

$\begin{array}{lll}(\$ 29.97) & (\$ 28.53) & (\$ 13.49)\end{array}$

$(\$ 11.23)$

18 PV+Lighting+Thermal

$19 \mathrm{kWh} / \mathrm{m} 2 / \mathrm{yr}$

$20 \$ / \mathrm{m} 2 / \mathrm{yr}$

21 Combinedpayback,years

\begin{tabular}{rrrrrrr}
99.81 & 5.18 & 5.18 & $(82.25)$ & $(41.93)$ & $(4.05)$ & $(26.48)$ \\
$\$ 17.50$ & $\$ 0.68$ & $\$ 1.41$ & $(\$ 7.38)$ & $(\$ 4.85)$ & $(\$ 0.29)$ & $(\$ 1.48)$ \\
\hline 44.09 & $1,280.41$ & 613.14 & $(93.59)$ & $(129.30)$ & $(2,544.86)$ & $(454.13)$
\end{tabular}

(183.46)

OPTION 1a 1

System cost, $\$ / m 2$

$\begin{array}{lllllll}\$ 407.33 & \$ 442.43 & \$ 442.43 & \$ 321.70 & \$ 310.24 & \$ 380.10 & \$ 339.97\end{array}$

3 PV output

$4 \mathrm{kWh} / \mathrm{m} 2 / \mathrm{yr}$

$5 \$ / \mathrm{m} 2 / \mathrm{yr}$

6 Simplepayback, years

\begin{tabular}{rrrrrrr}
156.46 & 111.77 & 111.77 & 184.37 & 141.79 & 124.58 & 117.26 \\
$\$ 27.43$ & $\$ 14.60$ & $\$ 30.48$ & $\$ 16.54$ & $\$ 16.42$ & $\$ 9.06$ & $\$ 6.57$ \\
\hline 14.85 & 30.31 & 14.51 & 19.45 & 18.90 & $\mathbf{4 1 . 9 5}$ & $\mathbf{5 1 . 7 1}$
\end{tabular}

27.38

OPTION 21 System cost, \$/m2

2 Additional HVAC, $\$ / m 2$

$\begin{array}{lllllll}\$ 947.23 & \$ 1,028.88 & \$ 1,028.88 & \$ 748.12 & \$ 721.46 & \$ 883.92 & \$ 790.61\end{array}$

3 Base system + HVAC

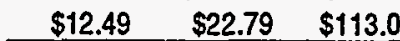

$\$ 35.78$

$\begin{array}{lllllll}\$ 959.73 & \$ 1,051.67 & \$ 1.141 .88 & \$ 783.91 & \$ 743.65 & \$ 904.71 & \$ 810.63\end{array}$

PV output

$\mathrm{kWh} / \mathrm{m} 2 / \mathrm{yr}$

$7 \$ / m 2 / y r$

8 PVonlypayback,years

\begin{tabular}{rrrrrrr}
154.99 & 110.89 & 110.89 & 182.43 & 140.44 & 123.53 & 116.30 \\
$\$ 27.17$ & $\$ 14.48$ & $\$ 30.24$ & $\$ 16.36$ & $\$ 16.26$ & $\$ 8.99$ & $\$ 6.52$ \\
\hline 34.86 & $\mathbf{7 1 . 0 4}$ & $\mathbf{3 4 . 0 2}$ & $\mathbf{4 5 . 7 2}$ & $\mathbf{4 4 . 3 7}$ & $\mathbf{9 8 . 3 7}$ & $\mathbf{1 2 1 . 2 5}$
\end{tabular}

64.23

10 Lighting savings

$11 \mathrm{kWh} / \mathrm{m} 2 / \mathrm{yr}$

$12 \$ / \mathrm{m} 2 / \mathrm{yr}$

61.64

$59.33 \quad 59.33$

67.54

62.65

$56.89 \quad 56.61$

13

14 Thermal savings (loss)

$15 \mathrm{kWh} / \mathrm{m} 2 / \mathrm{yr}$

$16 \$ / \mathrm{m} 2 / \mathrm{yr}$

$\$ 10.81$

$\$ 7.75$

$\$ 16.18$

$\$ 6.06 \$ 7.25$

$\$ 4.14$

$\$ 3.17$

17

18 PV+Lighting+Thermal

$19 \mathrm{kWh} / \mathrm{m} 2 / \mathrm{yr}$

$20 \quad \$ / \mathrm{m} 2 / \mathrm{yr}$

(14.12)

$\begin{array}{ll}(30.78) & (30.78)\end{array}$

$(78.51) \quad(51.80)$

(37.25)

(40.24)

(\$2.48)

(\$4.02) (\$8.39)

$(\$ 7.04) \quad(\$ 6.00)$

(\$2.71)

(\$2.26)

21 Combinedpayback,years

\begin{tabular}{rrrrrrr}
202.51 & 139.44 & 139.44 & 171.46 & 151.29 & 143.17 & 132.67 \\
$\$ 35.50$ & $\$ 18.21$ & $\$ 38.03$ & $\$ 15.38$ & $\$ 17.52$ & $\$ 10.41$ & $\$ 7.44$ \\
\hline 27.03 & 57.75 & 30.03 & 50.97 & 42.45 & 86.87 & 108.98
\end{tabular}

57.73

OPTION 2a 1 System cost, $\$ / m 2$

$\begin{array}{lllllll}\$ 446.73 & \$ 485.24 & \$ 485.24 & \$ 352.83 & \$ 340.26 & \$ 416.87 & \$ 372.87\end{array}$

2

3 PV output

$4 \mathrm{kWh} / \mathrm{m} 2 / \mathrm{yr}$

$5 \$ / \mathrm{m} 2 / \mathrm{yr}$

6 Simplepayback,years

\begin{tabular}{rrrrrrr}
154.99 & 110.89 & 110.89 & 182.43 & 140.44 & 123.53 & 116.30 \\
$\$ 27.17$ & $\$ 14.48$ & $\$ 30.24$ & $\$ 16.36$ & $\$ 16.26$ & $\$ 8.99$ & $\$ 6.52$ \\
\hline 16.44 & $\mathbf{3 3 . 5 1}$ & 16.04 & 21.56 & 20.92 & 46.39 & 57.18
\end{tabular}


PV1: 140W/ms @ \$4.40, Oakland New York1 New York2 Phoenix Mami Chicago Cincinnati. Av $\$ / k W h$ $\$ 0.175$

$\$ 0.131 \quad \$ 0.273 \quad \$ 0.090$

$\$ 0.116$

$\$ 0.073$

$\$ 0.056$

Construction cost location

1.14

1.24

$1.24 \quad 0.90$

0.87

$1.06-0.95$

OPTION 3

1
2
3
4
5
6
7
8
9
10
11
12
13
14
15
16
17
18
19
20
21

System cost, \$/m2

$\begin{array}{lllllll}\$ 678.97 & \$ 737.49 & \$ 737.49 & \$ 536.25 & \$ 517.14 & \$ 633.58 & \$ 566.70\end{array}$

4 PV output

$5 \mathrm{kWh} / \mathrm{m} 2 / \mathrm{yr}$

$6 \$$ im2/yr

PVonlypayback,years

\begin{tabular}{rrrrrrr}
158.42 & 112.94 & 112.94 & 186.94 & 143.59 & 125.96 & 118.53 \\
$\$ 27.77$ & $\$ 14.75$ & $\$ 30.80$ & $\$ 16.77$ & $\$ 16.63$ & $\$ 9.16$ & $\$ 6.65$ \\
\hline 24.45 & 50.00 & 23.94 & 31.98 & 31.11 & 69.15 & 85.28
\end{tabular}

45.13

Lighting savings

$11 \mathrm{kWh} / \mathrm{m} 2 / \mathrm{yr}$

$\$ / \mathrm{m} 2 / \mathrm{yr}$

0.00

0.00

0.00

0.00

0.00

$0.00 \quad 0.00$

$\$ 0.00$

$\$ 0.00$

$\$ 0.00 \$ 0.00$

$\$ 0.00 \quad \$ 0.00$

$\$ 0.00$

Thermal savings (loss)

$\mathrm{kWh} / \mathrm{m} 2 / \mathrm{yr}$

0.45

$\begin{array}{llll}0.53 \quad & 0.53 \quad 1.21\end{array}$

0.97

0.55

0.65

$\$ / \mathrm{m} 2 / \mathrm{yr}$

$\$ 0.08$

$\$ 0.07$

$\$ 0.14 \$ 0.11$

$\$ 0.11$

$\$ 0.04$

$\$ 0.04$

18 PV+Lighting+Thermal

$19 \mathrm{kWh} / \mathrm{m} 2 / \mathrm{yr}$

$\$ / \mathrm{m} 2 / \mathrm{yr}$

158.87

$\$ 27.8$

113.47

113.47

188.15

144.56

$126.51 \quad 119.18$

Combinedpayback,years

24.38

$\$ 14.82$

$\$ 30.95$

$\$ 16.88$

$\$ 16.74$

$\$ 9.20$

96.68

49.77

23.83

31.77

30.90

68.85

84.81

44.90

OPTION 4

1
2
3
4
5

System cost, \$/m2

$\$ 733.14 \quad \$ 796.33$

$\$ 796.33 \quad \$ 579.03$

$\$ 558.40 \quad \$ 684.13 \quad \$ 611.92$

Additional HVAC, $\$ / \mathrm{m} 2$

$\$ 17.85)$

9796.33

$\$ 16.14$

$\$(\$ 781)$

$\begin{array}{llll}(\$ 9.79) & (\$ 13.59) \quad(\$ 12.16)\end{array}$

$\begin{array}{lllllll}\$ 715.29 & \$ 780.19 & \$ 780.19 & \$ 571.22 & \$ 548.61 & \$ 670.54 & \$ 599.76\end{array}$

5

6

7 PV output

$8 \mathrm{kWh} / \mathrm{m} 2 / \mathrm{yr}$

$9 \$ / \mathrm{m} 2 / \mathrm{yr}$

10 PVonlypayback, years

\$715.29

$\$ 80.19$

(548.61

10.54

$\$ 627.42 \quad \$ 627.42 \quad \$ 456.21 \quad \$ 439.95 \quad \$ 539.02 \quad \$ 482.12$

11

12

13

Lighting savings

$\mathrm{kWh} / \mathrm{m} 2 / \mathrm{yr}$

$\$ 577.63$

9627.42

\begin{tabular}{rrrrrrr}
151.29 & 108.68 & 108.68 & 177.56 & 137.04 & 120.91 & 113.90 \\
$\$ 26.52$ & $\$ 14.19$ & $\$ 29.64$ & $\$ 15.93$ & $\$ 15.87$ & $\$ 8.80$ & $\$ 6.39$ \\
\hline 21.78 & $\mathbf{4 4 . 2 0}$ & 21.17 & $\mathbf{2 8 . 6 4}$ & $\mathbf{2 7 . 7 3}$ & $\mathbf{6 1 . 2 9}$ & $\mathbf{7 5 . 5 0}$
\end{tabular}

40.04

16

17 Thermal savings (loss)

18

19

20

$21 \mathrm{PV}+$ Lighting+Thermal

$22 \mathrm{kWh} / \mathrm{m} 2 / \mathrm{yr}$

$\mathrm{kWh} / \mathrm{m} 2 / \mathrm{yr}$

$\$ / m 2 / y r$

61.64

59.33

$\$ 10.81$

$\$ 7.75$

59.33

67.54

62.65

$\$ 16.18 \quad \$ 6.06$

$\$ 7.25$

$56.89 \quad 56.61$

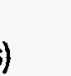

$23 \$ / \mathrm{m} 2 / \mathrm{yr}$

24

Combinedpayback,years

\begin{tabular}{rrrrrrr}
246.47 & 193.66 & 193.66 & 272.94 & 230.28 & 202.18 & 196.57 \\
$\$ 43.21$ & $\$ 25.29$ & $\$ 52.82$ & $\$ 24.48$ & $\$ 26.66$ & $\$ 14.71$ & $\$ 11.02$ \\
\hline $\mathbf{1 6 . 5 5}$ & $\mathbf{3 0 . 8 5}$ & $\mathbf{1 4 . 7 7}$ & $\mathbf{2 3 . 3 3}$ & $\mathbf{2 0 . 5 8}$ & $\mathbf{4 5 . 5 9}$ & $\mathbf{5 4 . 4 2}$
\end{tabular}

29.44 
PV1: 140W/ms @ \$4.40, Oakland New York1 New York2 Phoenix Mami Chicago Cincinnati $\begin{array}{llllllll}\text { Av } \$ \text { kWh } & \$ 0.175 & \$ 0.131 & \$ 0.273 & \$ 0.090 & \$ 0.116 & \$ 0.073 & \$ 0.056\end{array}$

\begin{tabular}{lllllllll} 
Construction cost location & 1.14 & 1.24 & 1.24 & 0.90 & 0.87 & 1.06 & 0.95 \\
\hline
\end{tabular}

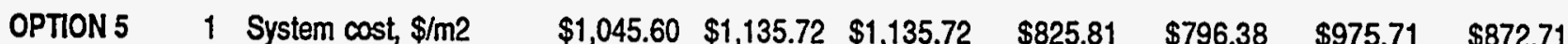

2 Additional HVAC, $\$ / m 2 \quad$\begin{tabular}{lllllll}
$(\$ 17.85)$ & $(\$ 16.14)$ & $(\$ 16.14)$ & $(\$ 7.81)$ & $(\$ 9.79)$ & $(\$ 13.59)$ & $(\$ 12.16)$ \\
\hline
\end{tabular}

$\begin{array}{lllllllll}3 & \text { Base system + HVAC } & \$ 1,027.75 & \$ 1,119.58 & \$ 1,119.58 & \$ 818.01 & \$ 786.60 & \$ 962.12 & \$ 860.56\end{array}$

4

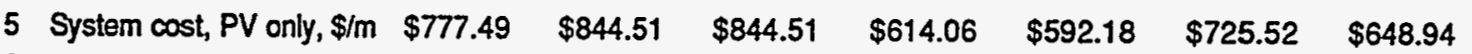

6

7 PV output

$\mathrm{kWh} / \mathrm{m} 2 / \mathrm{yr}$

$\$ / \mathrm{m} 2 / \mathrm{yr}$

PVonlypayback,years

\begin{tabular}{rrrrrrr}
157.92 & 112.64 & 112.64 & 186.28 & 143.13 & 125.60 & 118.20 \\
$\$ 27.69$ & $\$ 14.71$ & $\$ 30.72$ & $\$ 16.71$ & $\$ 16.57$ & $\$ 9.14$ & $\$ 6.63$ \\
\hline 28.08 & 57.41 & 27.49 & 36.75 & $\mathbf{3 5 . 7 3}$ & $\mathbf{7 9 . 4 1}$ & $\mathbf{9 7 . 9 2}$
\end{tabular}

Lighting savings

$\mathrm{kWh} / \mathrm{m} 2 / \mathrm{yr}$

$\begin{array}{llll}61.64 & 59.33 & 59.33 & 67.54\end{array}$

62.65

56.89

56.61

$\$ / \mathrm{m} 2 / \mathrm{yr}$

$\$ 10.81$

$\$ 7.75 \quad \$ 16.18 \quad \$ 6.06$

$\$ 7.25$

$\$ 4.14$

$\$ 3.17$

Thermal savings (loss)

$\mathrm{kWh} / \mathrm{m} 2 / \mathrm{yr}$

$\$ / m 2 / y r$

3.34
$\$ 0.59$

18.25

18.25

4.79

0.00

23.33

16.49

19

20

PV+Lighting+Thermal

$\mathrm{kWh} / \mathrm{m} 2 / \mathrm{yr}$

$\$ 2.38$

$\$ 4.98$

$\$ 0.43$

$\$ 0.00 \quad \$ 1.70$

$\$ 0.92$

$\$ / m 2 / y r$

2

22500

258.61

20578

Combinedpayback,years

$\begin{array}{lll}26.08 & \$ 24.84 & \$ 51.88\end{array}$

$\$ 23.20$

$\$ 23.83$

205.82

191.30

$\begin{array}{lll}45.07 & 21.58 & 35.26\end{array}$

33.01

$\$ 14.97$

$\$ 10.73$

Max thermal savings (loss)

$\mathrm{kWh} / \mathrm{m} 2 / \mathrm{yr}$

$\$ / \mathrm{m} 2 / \mathrm{yr}$

$77.62 \quad 74.70$

74.70

124.56

94.15

$64.26 \quad 80.24$

43.67

28

29 PV+Lighting+Max thermal

$30 \mathrm{kWh} / \mathrm{m} 2 / \mathrm{yr}$

$\$ 13.61$

$\$ 9.76 \quad \$ 20.37$

$\$ 11.17 \$ 10.90$

74.52

75.95

$\$ 5.42 \quad \$ 4.26$

$31 \$ / m 2 / y r$

297.18

246.67

246.67

378.38

299.93

257.01

250.76

$\$ 52.10 \quad \$ 32.21$

$\$ 67.27$

$\$ 33.94$

$\$ 34.73$

$\$ 18.70$

$\$ 14.06$

32.94 


\section{B BUILDING ENERGY BALANCE MODEL: PARAMETERS AND EQUATIONS}

The building load data provided in this study was calculated from a building energy balance computer model developed by Mahadev Raman at Ove Arup \& Partners, Engineers. The following is a brief discussion of the parameters assumed and equations used in calculating the energy loads for each of the five roof options in six cities. Detailed energy balance calculation spreadsheets for each option are provided in the appendix following this discussion. 
The configuration assumed for analysis is a single story space with a PV array integrated into the roof. The PV array therefore exchanges heat directly with the occupied space.

A single square meter of roof and corresponding floor space is used for analysis. It is assumed that the only direct heat exchange with the external environment is through the roof. Floor gains and losses are ignored as are perimeter conditions. The effective error this introduces to the analysis depends on multiple factors: overall building size and shape (i.e. floor-to-roof ratio), facade materials, the amount of perimeter windows, building use, climatic variations, etc. Since the perimeter of the case study building is small relative to roof area, it is assumed that the primary energy gains and losses are determined by roof conditions. Detailed design of supplemental building conditions is not considered in the scope of this study.

The temperature within the space is assumed to be at a constant $21^{\circ} \mathrm{C}$ during occupied hours but is allowed to drift by up to $\pm 5^{\circ} \mathrm{C}$ when the building is unoccupied.

The following heat gains are assumed:

$\begin{array}{ll}\text { People } & 20 \mathrm{~W} / \mathrm{m} 2(\sim 500 \text { lux }) \\ \text { Lights } & 20 \mathrm{~W} / \mathrm{m} 2(\sim 500 \text { lux }) \\ \text { Equipment } & 10 \mathrm{~W} / \mathrm{m} 2(\sim 250 \text { lux })\end{array}$

These heat gains are assumed to be constant during occupied hours. When the building is unoccupied, gains of $4.5 \mathrm{~W} / \mathrm{m} 2$ are assumed to take account of security lighting and equipment.

A fresh air provision of 2 liters/s per $\mathrm{m} 2$ is taken into account and corresponds to 10 liters/s per person.

As the model for this analysis is a convention center, a seven-days-per-week and ten-hours-per-day occupancy profile is assumed.

On the whole, these assumptions are conservative and can be expected to over estimate energy consumption.

Climatic data have been obtained for the following cities:

- Oakland

- New York City 
- Phoenix

- Miami

- Chicago

- Cincinnati

Together, these cities cover the majority of climate types found in the continental USA.

The following items of weather data are used in the analysis:

- Average daily maximum and minimum temperatures for each month (TMX, TMN).

- Corresponding relative humidities ( $R M X, R M N)$.

- Monthly hours of bright sunshine (HS).

- Theoretical 24 hour average solar radiation figures for each month based on latitude and climate type (IAV).

These are manipulated to create four analysis conditions for each month:

1. Sunny day occupied period

2. Sunny day unoccupied period

3. Cloudy day occupied period

4. Cloudy day unoccupied period

Each month is divided into equivalent sunny and cloudy days as follows:

$\mathrm{NS}=\mathrm{HS} / \mathrm{HD}$ and $\mathrm{NC}=\mathrm{N}-\mathrm{NS}$

where

NS = number of sunny days in the month

HS = hours of bright sunshine in the month

$\mathrm{HD}=$ hours of daylight per day in the month

$\mathrm{NC}=$ number of cloudy days in the month

$\mathrm{N}=$ total days in the month

The 24 hour average direct and diffuse solar radiation figures were obtained from tables in the CIBSE (Chartered Institution Building Services Engineers, United Kingdom) guide. All solar radiation is assumed to occur evenly over the daylight hours. The occupied period is assumed to occur during daylight hours. Radiation occurring in daylight hours outside the occupied period is spread evenly over the unoccupied hours.

$\mathrm{IO}=(\mathrm{IAV} \times 24) / \mathrm{HD}$

where

$\mathrm{IO}=$ occupied hours solar intensity $\mathrm{W} / \mathrm{m} 2$

$\mathrm{IAV}=24$ hour average solar intensity $\mathrm{W} / \mathrm{m} 2$

$\mathrm{HD}=$ hours of daylight per day 
$\mathrm{IU}=\mathrm{IO} \times(\mathrm{HD}-\mathrm{HO}) / \mathrm{HU}$

where

$\mathrm{IU}=$ unoccupied hours solar intensity $\mathrm{W} / \mathrm{m} 2$

$\mathrm{HO}=$ occupied hours per day

$\mathrm{HU}=$ unoccupied hours per day

IAV for sunny days is calculated from the sum of the theoretical direct and diffuse radiation components taking into account factors related to the climate type:

$\mathrm{IAV}=(\mathrm{ID} \times \mathrm{RD})+(\mathrm{Id} \times \mathrm{Rd})$

where

$\mathrm{ID}=24$ hour average direct radiation $\mathrm{W} / \mathrm{m} 2$

$\mathrm{Id}=24$ hour average diffuse radiation $\mathrm{W} / \mathrm{m} 2$

$\mathrm{RD}=$ direct radiation factor

$\mathrm{Rd}=$ diffuse radiation factor

On cloudy days:

$\mathrm{IAV}=\mathrm{Id}$

The external temperature during occupied and unoccupied periods are approximated as follows:

$\mathrm{TO}=(\mathrm{TMX}+\mathrm{TAV}) / 2$

$\mathrm{TU}=[(\mathrm{TAV} \times 24)-(\mathrm{TO} \times \mathrm{HO})] / \mathrm{HU}$

where

$\mathrm{TAV}=$ the average of $\mathrm{TMX}$ and $\mathrm{TMN}{ }^{\circ} \mathrm{C}$

$\mathrm{TO}=$ temperature during occupied hours ${ }^{\circ} \mathrm{C}$

$\mathrm{TU}=$ temperature during unoccupied hours ${ }^{\circ} \mathrm{C}$

\subsection{ANALYSIS MODEL}

The analysis model carries out steady state thermal balance calculation for the four analysis conditions defined above for each month.

At the roof, the equations governing energy transfers are as follows:

$Q L=U(T i-T o)-a R 3 U I$ 
where

$\mathrm{QL}=$ conduction loss $\mathrm{W} / \mathrm{m} 2$

$\mathrm{U}=$ thermal transmittance $\mathrm{W} / \mathrm{m} 2 \mathrm{~K}$

$\mathrm{Ti}=$ inside temperature ${ }^{\circ} \mathrm{C}$

To $=$ outside temperature ${ }^{\circ} \mathrm{C}$

$\mathrm{a}=$ absorbtivity

. R3 = external surface resistance $\mathrm{m} 2 \mathrm{~K} / \mathrm{W}$

$\mathrm{I}=$ solar intensity $\mathrm{W} / \mathrm{m} 2$

$\mathrm{QT}=\mathrm{t} \mathrm{I}-\mathrm{QL}$

where

QT = total heat gain $W / \mathrm{m}^{2}$

$t=$ transmittivity

For each month the resulting energy transfer is summed with the appropriate level of internal heat gains to determine the net heat gain or loss in each of the four analysis condition (sunny/cloudy, occupied/unoccupied). Gains due to lighting are reduced when appropriate to account for the use of natural light.

The overall heating and cooling requirements in $\mathrm{kWh}$ are then calculated by multiplying the instantaneous gains and losses by the number of hours in each period.

The electrical energy consumption for heating and cooling is calculated by using a coefficient of performance of 3.5 for cooling and 4.5 for heating. This assumes a reverse cycle heat pump based system. Full credit is given for free outside air cooling when this is available.

Fan energy consumption is calculated assuming a constant volume system sized to match the peak heat gain. Electricity consumed by equipment and artificial lighting is also taken into account.

Fresh air heating and cooling requirements (needed during occupied hours only) are determined using an enthalpy difference calculation where the outdoor enthalpy is determined from the outdoor temperature and humidity. The indoor enthalpy is based on the internal temperature and humidity. This humidity is allowed to vary between upper and lower limits to minimize energy consumption. Again, the heating and cooling of the outside air is by means of a reverse cycle heat pump system using the coefficients of performance defined above. 


\section{BUILDING ENERGY BALANCE CALCULATIONS}




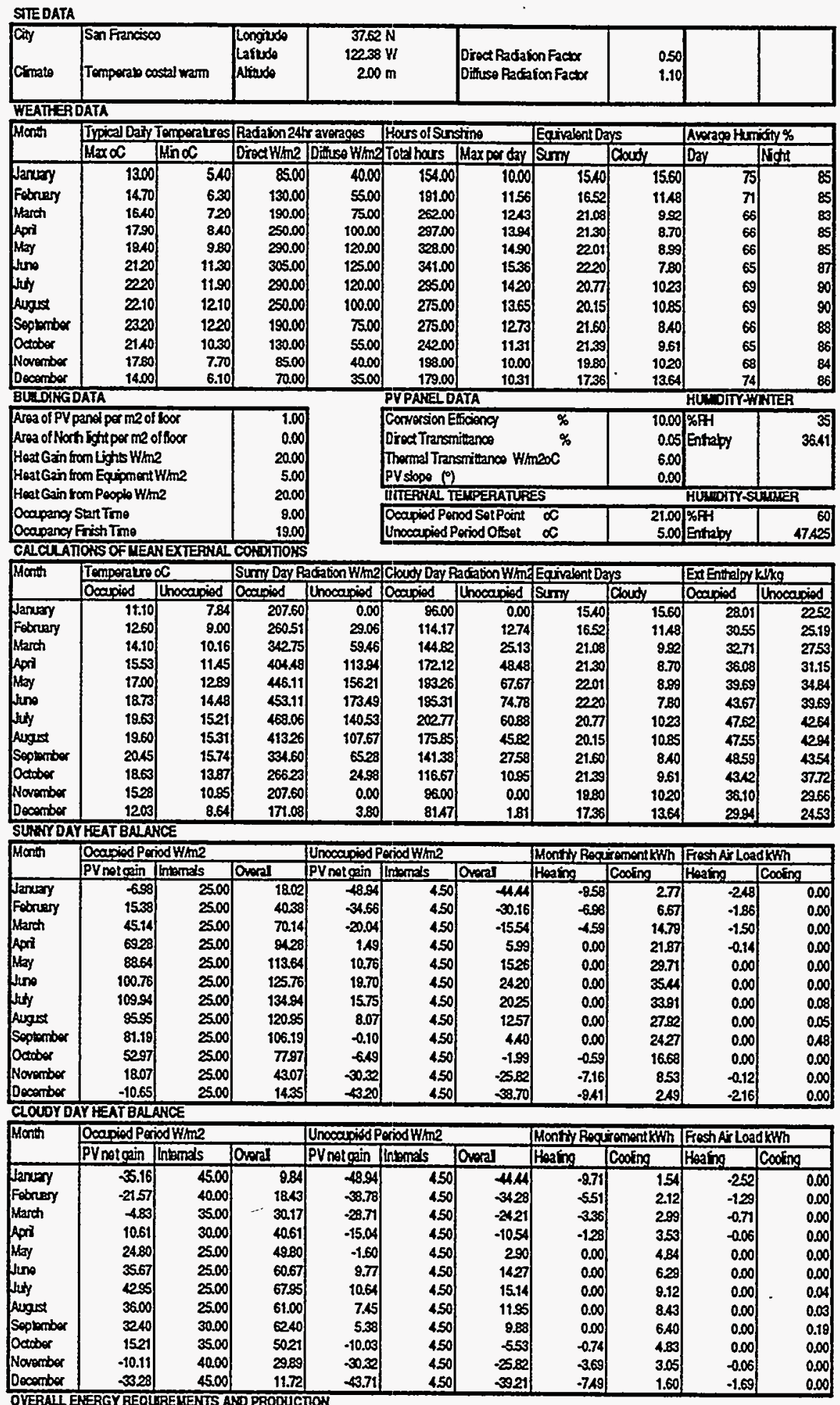

OVERALL ERERGY REQLAREMENTS AWD PROOUCIOW

\begin{tabular}{|c|c|c|c|c|c|c|c|}
\hline \multirow[t]{2}{*}{ Manth } & \multicolumn{2}{|c|}{ Coding Consumpion } & \multicolumn{2}{|c|}{ Heating Consumetion } & \multirow{2}{*}{$\begin{array}{l}\text { Fans } \\
\text { kish }\end{array}$} & \multirow{2}{*}{$\begin{array}{l}\text { Lighing and } \\
\text { Powst kth }\end{array}$} & \multirow{2}{*}{$\begin{array}{l}\text { Tobl kwh } \\
\text { Corsemed }\end{array}$} \\
\hline & 11000 & then Eectic & $1 / 1000$ & Whin Eectic & & & \\
\hline Januory & 0.00 & 0.00 & 0.22 & 5.40 & 9.58 & 5.76 & 20.73 \\
\hline Fobruay & 0.00 & 0.00 & 022 & 3.48 & 8.55 & 4.10 & 1623 \\
\hline Merch & 0.00 & 0.00 & 0.22 & 226 & 958 & 3.63 & 15.46 \\
\hline Apri & 029 & 726 & 022 & 0.33 & 8.27 & 2.99 & 1984 \\
\hline May & 0.20 & 9.87 & 022 & 0.00 & 0.58 & 264 & 2209 \\
\hline Jino & 0.29 & 11.92 & 0.22 & 0.00 & 827 & 255 & 23.74 \\
\hline Juty & 0.29 & 1233 & 022 & 0.00 & 858 & 264 & 2454 \\
\hline August & 029 & 10.41 & 022 & 0.00 & 8.58 & 2.64 & 202 \\
\hline Sepenter & 0.29 & 8.95 & 022 & 0.00 & 827 & 297 & 21.19 \\
\hline October & 028 & 6.14 & 022 & 0.30 & 8.58 & 3.60 & 18.2 \\
\hline Novertber & 028 & 3.31 & 0.22 & 245 & 927 & 4.08 & 19.11 \\
\hline Decontiber & 0.00 & 0.00 & 022 & 4.61 & 8.58 & 5.36 & 1955 \\
\hline TOTALm2 & & 70.19 & & 18.82 & 112.78 & 4293 & 244.73 \\
\hline
\end{tabular}




\begin{tabular}{|c|c|c|c|c|c|c|c|c|c|c|}
\hline $\begin{array}{l}\text { Caty } \\
\text { Climato }\end{array}$ & $\begin{array}{l}\text { New York } \\
\text { Temperate cos }\end{array}$ & $\operatorname{cosed}$ cold & $\begin{array}{l}\text { Longude } \\
\text { Laitudo } \\
\text { Afturdo }\end{array}$ & $\begin{array}{l}40.701 \\
74.021 \\
3.00 \mathrm{I}\end{array}$ & $\begin{array}{l}O N \\
2 W \\
O M\end{array}$ & $\begin{array}{l}\text { Droct Radase } \\
\text { Difiso Radia }\end{array}$ & $\begin{array}{l}\text { ion Factor } \\
\text { ifon Factor }\end{array}$ & $\begin{array}{l}0.50 \\
1.10\end{array}$ & & \\
\hline WEATHER & DATA & & & & & & & & & \\
\hline Mon' & Trpical Dary T & Temosatres & Radaion 24 & ravereos If & Hous of Sins & $\sin \theta$ & Egriviont Da & & Alorag Him & nidit\% \\
\hline & $\operatorname{Max} O C$ & $\operatorname{Min} \alpha$ & Dract W/m2 & Dimuso What & Toka hars & Thaxperday & Serny & County & Day & Nigh \\
\hline January & 430 & -2600 & 8500 & 40.00 & 151.00 & 10.00 & 15.10 & 1590 & 61 & 69 \\
\hline Fabreary & 4.10 & -240 & 130.00 & 55,00 & 164.00 & 1028 & 1586 & 1204 & 58 & 67 \\
\hline March & 8.90 & 0.60 & 190.00 & 75.00 & 200.00 & 1183 & 17.67 & 1333 & 56] & 67 \\
\hline Apri & 14900 & 6.10 & 250.00 & 100000 & 21400 & 1208 & 17.70 & 1230 & 5 & 68 \\
\hline May & 20.00 & 1180 & 200.00 & 120.00 & 250.00 & 1344 & 1860 & 1240 & 56 & $\infty$ \\
\hline $\operatorname{Jin} \theta$ & 2560 & 1690 & 305.00 & 125.00 & 301.00 & 16.18 & 18.60 & 1110 & 58 & 72 \\
\hline Juty & 2830 & 19.90 & 20000 & 120.00 & 307.00 & 1500 & 20.46 & 1054 & 57 & 74 \\
\hline August & 2740) & 1930 & 250.00 & 100.00 & 275.00 & 13.86 & 1984 & 11.16 & 60 & 76 \\
\hline Septrmber & 2380 & 1580 & 190.00 & 75.00 & 237.00 & 1254 & 1890 & 11.10 & 60 & 76 \\
\hline loctobar & 18.40 & 1030 & 130.00 & 55.00 & 218.00 & 1134 & 1922 & 11.78 & 59 & 74 \\
\hline November & 11.90 & 440 & 8500 & 40,00 & 17200 & 10.06 & 17.10 & 1290 & 60 & 71 \\
\hline December & 5.70 & -120 & 70.00 & 35.00 & 158.00 & 10.00 & 1580 & 1520 & 60 & 67 \\
\hline BLIOING & DATA & & & & PVPARED D & DATA & & & RLWDTYभI & VIIER \\
\hline $\begin{array}{l}\text { Area of PV } \\
\text { Aroa of Nor } \\
\text { Heat Gain } 6 \\
\text { Heat Gain }\end{array}$ & $\begin{array}{l}\text { and por m2 of } \\
\text { h Ight por m2 of } \\
\text { on Lights W/m2 } \\
\text { on Eqigment }\end{array}$ & $\begin{array}{l}\text { Thor } \\
\text { ol boot } \\
2 \\
\text { Whon }\end{array}$ & $\begin{array}{r}1.00 \\
0.00 \\
20.00 \\
5.00\end{array}$ & & $\begin{array}{l}\text { Converson E } \\
\text { Droct Transm } \\
\text { Thermal Tran } \\
\text { PV stope (9) }\end{array}$ & $\begin{array}{l}\text { Eficioncy } \\
\text { anitanos } \\
\text { nesrituanco W/h } \\
\text { ) }\end{array}$ & $m=c^{\%}$ & $\begin{array}{r}10.00 \\
0.05 \\
6.00 \\
0.00\end{array}$ & $\begin{array}{l}\text { EFF } \\
\text { Entratby }\end{array}$ & $\begin{array}{r}35 \\
3641\end{array}$ \\
\hline HatGinf & on Peoplo Whe & & 20.00 & & WNERWAL IT & IELPEAATR: & & & HLLADITYSU & AIUER \\
\hline $\begin{array}{l}\text { Ocapancy } \\
\text { Ocapancy }\end{array}$ & $\begin{array}{l}\text { StatTme } \\
\text { Frish Tme }\end{array}$ & & $\begin{array}{r}9.00 \\
19.00\end{array}$ & & $\begin{array}{l}\text { Ocapiod Pen } \\
\text { UnocapiedP }\end{array}$ & $\begin{array}{l}\text { eriod SetPoint } \\
\text { Period Otset }\end{array}$ & $\begin{array}{l}\alpha \\
\alpha\end{array}$ & $\begin{array}{r}21.00 \\
5.00\end{array}$ & $\begin{array}{l}\text { Xory } \\
\text { Entraby }\end{array}$ & $\begin{array}{r}60 \\
47 A 25 \\
\end{array}$ \\
\hline CALCULAT & 1ONS OFMEAH & WEXTERYLL & COADITIONK & & & & & & & \\
\hline Month & Temperatroo & & Surny DayR & 26abon Wimze & JChoudy Oay $\mathrm{R}$ & Racabon Whm & Equabent $\mathrm{Ds}_{3}$ & & Extentrabyk & Klikg \\
\hline & Docopiod I & Chocapiod & Ocospiod & (chocapiod & Docapiod & Unocamiod & $\operatorname{sen} y$ & Cacoly & ocaniod I & Uhoos piod \\
\hline Jaxery & 253 & .0 .52 & 207.50 & 0.00 & 8600 & 0.00 & 15.10 & 1590 & 8.5 & 4.83 \\
\hline Fabruary & $2 A B$ & -031 & 253.12 & 5.7 & $128 \mathrm{A6}$ & 253 & 1595 & 1204 & 8.56 & 4.99 \\
\hline March & 6.83 & 327 & 360.16 & 47.03 & 152.18 & 19.87 & 17.67 & 1333 & 1567 & 10.86 \\
\hline & 1270 & 8.93 & 466,19 & 69.65 & 188.50 & 29.64 & 17.70 & 1230 & 2677 & 2180 \\
\hline May & 18.03 & 14.73 & 496.61 & 121.56 & 21427 & 5266 & 18.60 & 1240 & 39.98 & 35.05 \\
\hline Jine & 2343 & 19.70 & 430.09 & 169.94 & 18538 & 81.87 & 18.60 & 1140 & 5274 & 49.08 \\
\hline Juty & 2620 & 2260 & 43.06 & 15839 & 191.24 & 68.62 & 20.46 & 10.54 & 59.60 & 5933 \\
\hline furgest & 2538 & 2190 & 406.20 & 11221 & 173.15 & 47.75 & 19.84 & 11.16 & 59.06 & 57.18 \\
\hline Sepromber & 2180 & 1837 & 339.72 & 61.63 & 143.54 & 26.04 & 1890 & 11.10 & 49.48 & 4690 \\
\hline Ootober & 1638 & 1290 & 265.55 & 25.46 & 11638 & 11.16 & 1922 & 11.78 & 3584 & 3204 \\
\hline November & 10.03 & 681 & 20639 & 0.86 & 8544 & $0 A 0$ & 17.10 & 1200 & 2215 & 1801 \\
\hline Decembor & 3.98 & 1.02 & 176,10 & 0.00 & 84.00 & 0.00 & 15.80 & 1520 & 1126 & 7.09 \\
\hline SUSWY DA & YHEAT BALAN & $N C E$ & & & & & & & & \\
\hline Manth & Doopiod Peric & riod Whm2 & & UnocapiodP & PoriodW/m2 & & Mont & Ii enent kth & Frest ArLoad & adkwh \\
\hline & PYnet gain II & Intand's & Omaral & PVnotgin I & Intomals & Tomal & Heaing & Cooing & Heating & Cooting \\
\hline Jantery & $-58,43$ & 25.00 & .33 .43 & $-\infty .11$ & 450 & -94.61 & -20.00 & 5.05 & .796 & 0.00 \\
\hline Fabreary & -37.14 & 2500 & -12.14 & -86.41 & 450 & -91.91 & -20.54 & -1.94 & -854 & 0.00 \\
\hline March & 588 & 2500 & 30.89 & 6452 & 450 & $+\infty .02$ & -14.85 & 5.46 & -7.04 & 0.00 \\
\hline Aprit & 6799 & 2500 & 8209 & -24.84 & 450 & -2034 & -5.04 & 1646 & 330 & 0.00 \\
\hline May & 110.64 & 2500 & 135.64 & -696 & 450 & -246 & -0.64 & 2523 & 0.00 & 0.00 \\
\hline Juno & 123.15 & 2500 & 148.15 & 20.14 & 450 & 24.64 & 0.00 & 33.97 & 0.00 & 1.90 \\
\hline Wuty & 143.07 & 2500 & 168.07 & 19.59 & 450 & 24.09 & 0.00 & 1129 & 0.00 & 438 \\
\hline Angast & 128.99 & 2500 & 153.99 & 1376 & 4.50 & 1826 & 0.00 & $35 \infty$ & 0.00 & 4.43 \\
\hline Sepumber & 90.58 & 25.00 & 115.58 & -021 & 450 & 429 & 0.00 & 2208 & 0.00 & -0.75 \\
\hline October & 39.30 & 2500 & 6430 & $-12,15$ & 450 & -7.65 & -206 & 1236 & $-02 t$ & 0.00 \\
\hline Norembor & -13.74 & 2500 & 1126 & 54.22 & 450 & 50,12 & -12.07 & 1.93 & 458 & 0.00 \\
\hline Decomber & .57 .61 & 25.00 & 32.81 & -29.89 & 450 & $-\infty 39$ & -18.69 & -5.15 & -7.63 & 0.00 \\
\hline CLOWOYD & AY HEAT BALA & AKCE & & & & & & & & \\
\hline Manth & Docapiod Pen & nodWIm2 & & Unnocapoid P & Period Wh/n2 & & Monty Rogi & Fomentkinh & Ficosh ArL Lor & dkth \\
\hline & PVnotcain & lnkme's & Overal & PVnet gain 1 & Intomals & Toveral & Hoaling & Coofing & Heaing & $\operatorname{cosing}$ \\
\hline January & $-\infty 6.61$ & 45.00 & -41.61 & $-\infty .11$ & 450 & -84.61 & -21.06 & -6.62 & -838 & 0.00 \\
\hline Fobreary & -78.71 & 40.00 & $-\$ 3.71$ & -9723 & 450 & -82.73 & -15.63 & 466 & -6.4 & 0.00 \\
\hline March & -46.62 & 35.00 & -11.20 & -71.38 & 4.50 & $+\infty 88$ & -1248 & -155 & -531 & 0.00 \\
\hline Aprit & 0.32 & 30.00 & 30,32 & 3494 & 450 & $-20 A$ & 524 & 3.73 & .229 & 0.00 \\
\hline May & 39.85 & 25.00 & 64.85 & 5.65 & 450 & 10.15 & 0.00 & 8.80 & 0.00 & 0.00 \\
\hline Jino & 6136 & 2500 & 8636 & 9.85 & 450 & 1435 & 0.00 & 1214 & 0.00 & 1.16 \\
\hline Juty & 79.66 & 25.00 & 104.66 & 11.93 & 450 & 1643 & 0.00 & 1345 & 0.00 & 246 \\
\hline Augast & 69.97 & 25.00 & 94.97 & 7,48 & 450 & 11.58 & 0.00 & 1247 & 0.00 & 249 \\
\hline Soptember & 41.04 & 30.00 & 71.04 & -620 & 450 & -1.70 & -026 & 720 & 0.00 & 0.44 \\
\hline petaber & 1.64 & 35.00 & 36.64 & -15.76 & 450 & -1126 & -1.86 & 432 & 0.13 & 0.00 \\
\hline November & 41.75 & 40.00 & -1.75 & 504 & 450 & -5054 & -9.13 & -023 & 246 & 0.00 \\
\hline Decermber & .0094 & 45.00 & -3.94 & $-\infty 80$ & 4.50 & -85.39 & -18.17 & 5.46 & .734 & 0.00 \\
\hline OVERAII & EKERGYREQU & UPREMFTS A & APDPROOUC & CTIOH & & & & & & \\
\hline Month & Cooing Const & sumplion & Heating Cons & sumobion & J Fans & Lighing and & TotalkWh & & & \\
\hline & 11000 & krm Eoctic & $1 / 100$ & kWh Eoctic & kWh & Power kth & Consumed & & & \\
\hline January & 0.00 & 0.00 & 0.22 & 1276 & 1128 & 5.82 & 2986 & & & \\
\hline Fabruay & 0.00 & 0.00 & 022 & 1136 & 10.19 & 4.19 & 25.74 & & & \\
\hline March & 0.00 & 0.00 & 022 & 8.82 & 1128 & 3.97 & 24.07 & & & \\
\hline Apri & 0.00 & 0.00 & 022 & 3.53 & 10.92 & 3.17 & 17.61 & & & \\
\hline May & 020 & 10.01 & 0.22 & 0.14 & 1128 & 2.54 & 24.07 & & & \\
\hline $\sin \theta$ & 0.29 & 14.05 & 022 & 0.00 & 10.28 & 255 & 27.52 & & & \\
\hline Juty & 0.29 & \$7.71 & 022 & 0.00 & 1128 & 2.64 & 31.60 & & & \\
\hline August & 0.20 & 15.72 & 0.22 & 0.00 & 1128 & 264 & 29.64 & & & \\
\hline Soptombor & 0.20 & 9.16 & 0.22 & 0.06 & 10.92 & 3.11 & 2324 & & & . \\
\hline Octaber & 0.29 & 4.76 & 022 & 0.95 & 1128 & 381 & 20.81 & & & \\
\hline Novembar & 0.00 & 0.00 & 0.22 & 650 & 10.52 & 4,49 & 21.00 & & & \\
\hline Decombor & 0.00 & 0.00 & 022 & 1156 & 1128 & 5.68 & 2852 & & & \\
\hline TOTALIm2 & & 71A1 & & 55.67 & 13286 & 44.67 & 304.61 & & & \\
\hline
\end{tabular}


OPTION 1: SEMLTRANSPARENT SINGLE-GLAZED PV ROOF

\begin{tabular}{|c|c|c|c|c|c|c|c|c|c|c|}
\hline $\begin{array}{l}\text { Cay } \\
\text { Cinate }\end{array}$ & $\begin{array}{l}\text { Phoerix } \\
\text { Temperato aid }\end{array}$ & & $\begin{array}{l}\text { Longudes } \\
\text { laituda } \\
\text { Alindo }\end{array}$ & $\begin{array}{r}33,43 \\
11202 \\
340,00\end{array}$ & & $\begin{array}{l}\text { Direct Radiafion } \\
\text { Dituso Racio }\end{array}$ & $\begin{array}{l}\text { on Factor } \\
\text { ion Factor }\end{array}$ & $\begin{array}{l}1.10 \\
0.90\end{array}$ & & \\
\hline WEATIER & ATA & & & & & & & & & \\
\hline Manth & Typical Daiy T & Temperatros & Radation 24x & avarages & Hours of Sunst & sing & Eativalont Days & & Averaco Humi & 6ry $\%$ \\
\hline & $\operatorname{Max} O C$ & Minoc & Droct Wime & Dillinso Whin2 & Tot hours & Juaxper day & sumy & Coody & Day & Ningt \\
\hline Jankey & 17880 & 180 & 105.00 & 25.00 & 25200 & 10.84 & 24.18 & 682 & 4 & 72 \\
\hline Fobruay & 20.10 & 380 & 150.00 & 30.00 & 271.00 & 1241 & 21.84 & 6.16 & 42 & 71 \\
\hline March & 2390 & 6.10 & 205.00 & 3500 & 331.00 & 1256 & 2635 & 4.65 & 31 & 60 \\
\hline Apri & 28.80 & 1020 & 260.00 & 4500 & 350.00 & 1348 & 2670 & 3.30 & 26 & 50 \\
\hline May & 33.80 & 1390 & 295.00 & 4500 & 408.00 & 14.00 & 29.14 & 188 & 19 & 41 \\
\hline Jino & 38.70 & 1860 & 305.00 & 5000 & 411.00 & 1457 & 2820 & 180 & 17 & 37 \\
\hline Suly & 40.30 & 23.90 & 255.00 & 4500 & 300.00 & 1430 & 2728 & 3.72 & 29 & 51 \\
\hline Augs: & 38.70 & 23.00 & 260.00 & 4500 & 379.00 & 1422 & 26.66 & 434 & 35 & 60 \\
\hline September & 3680 & 19.60 & 205.00 & 3500 & 354.00 & 13.11 & 2700 & 300 & 20 & 66 \\
\hline Octabor & 30.40 & 1260 & 150.00 & 30,00 & 323.00 & 11.88 & 25:97 & 403 & 30 & 58 \\
\hline Noveriber & 2320 & 5.80 & 105.00 & 2500 & 282.00 & 10.93 & 2580 & 420 & 33 & 23 \\
\hline Decomber & 1890 & 280 & 90.00 & 25.00 & 260.00 & 10.75 & 24.18 & $6 . \mathrm{B} 2$ & 42 & 70 \\
\hline BUIDIKGD & ATA & & & & PVPANELDA & ATA & & & 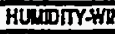 & NTER \\
\hline $\begin{array}{l}\text { Area of PV } \\
\text { Aroz of Nor } \\
\text { Heat Gain th } \\
\text { HeatGain tr }\end{array}$ & 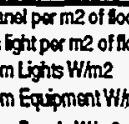 & $\begin{array}{l}\text { loor } \\
\text { toor } \\
\text { the }\end{array}$ & $\begin{array}{r}1.00 \\
0.00 \\
20.00 \\
5.00\end{array}$ & & $\begin{array}{l}\text { Conversion ES } \\
\text { Oroct Transmi } \\
\text { Thermal Transt } \\
\text { PV siopo ( }\end{array}$ & 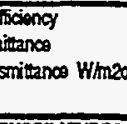 & $20 c^{\%}$ & \begin{tabular}{r|}
10.00 \\
0.05 \\
6.00 \\
0.00 \\
\end{tabular} & $\begin{array}{l}\text { FHA } \\
\text { Entrapy }\end{array}$ & $\begin{array}{r}35 \\
3641\end{array}$ \\
\hline Heat Gain 1 & m Peoplo W/m2 & & 20.00 & & INIEPNAL IE & EMPERATLPES & & & HUDMOTYSU & JWER \\
\hline $\begin{array}{l}\text { Ocapancy } \\
\text { Ocospanoy }\end{array}$ & Thish Tme & & $\begin{array}{r}9.00 \\
18.00\end{array}$ & & $\begin{array}{l}\text { Oocupiod Perio } \\
\text { Unocouniod Pe }\end{array}$ & $\begin{array}{l}\text { iod Solpoint } \\
\text { ecriod Onsot }\end{array}$ & $\begin{array}{l}\alpha \\
\alpha\end{array}$ & $\begin{array}{r}21.00 \\
5.00\end{array}$ & EFH & $\begin{array}{r}60 \\
47 \times 25\end{array}$ \\
\hline CALCILAT & OLS OF MEAPE & DXTERAL $\infty$ & OKOITROAS & & & & & & & \\
\hline Mandh & Temperatureod & & Sumy Day Rad & fabonW/m2 & Cloudy Day Paz & actabonW/m2 & Equivalent Days & & ExtEntriloyk & \\
\hline & Dosupiod & Thoseaniod & Doapiod & Uhocanied & locapiod & Turocapiod & sumy & Coury & Ooxaiod & Thosaniod \\
\hline January & 1380 & 6.94 & 305.66 & 1824 & 419.75 & 6.69 & 24.18 & 6.82 & 2594 & 1843 \\
\hline Fabruay & 16.03 & 9.04 & 37136 & 608 & 116.05 & 1890 & 21.84 & 6.16 & 2956 & 2250 \\
\hline March & 19.45 & 11.82 & 491.02 & 8984 & 139.74 & 24,47 & 26.35 & 1.55 & 3120 & 2613 \\
\hline Apxi & 24.15 & 16.18 & 581.17 & 144.59 & 16020 & 3986 & 26.70 & 330 & 37.82 & 3246 \\
\hline May & 2883 & 2030 & 025.65 & 17882 & 15427 & 4.09 & 29.14 & 286 & 4152 & $37 \times 2$ \\
\hline Do & 33.68 & 2506 & 625.58 & 204.73 & 164.67 & 5381 & 2820 & 180 & 47.87 & 45.9 \\
\hline Juty & 3620 & 29.17 & 612.75 & 18304 & 151.09 & 4636 & 2728 & 3.72 & 02.45 & 6382 \\
\hline Augus: & 34.78 & 28.05 & 55121 & 165.99 & 151.94 & 45.76 & 26.66 & 434 & 65.11 & 66.63 \\
\hline Septonber & 3250 & 25.13 & $470 A 4$ & 10454 & 128.14 & 2847 & 27.00 & 3.00 & 55.49 & 61.70 \\
\hline Octaber & 2595 & 1832 & 384.76 & 5431 & 12024 & 16.97 & 26.97 & 403 & 4331 & 40.03 \\
\hline November & 1885 & 1139 & 303.01 & 20.13 & 109.79 & 729 & 25800 & 420 & 31.61 & 30.54 \\
\hline Docember & 14.88 & 7.58 & 271.19 & 14.58 & 111.60 & 6.00 & 24.18 & 6.80 & 2738 & 20.20 \\
\hline SUXAY DA & HEAT BALANC & & & & & & & & & \\
\hline Manth & Toespiad Perio & $00 \mathrm{~W} / \mathrm{m} 2$ & & Unoocupiod Pe & PeriodW/m? & & Monthy Rogure & cementkwh & Fresh Ar Load & dkwh \\
\hline & Pynotoain & Theome's & Toval & PVnotgin & Inomals & Oreral & Hoaing & $\operatorname{cosing}$ & Hoafm & $\cos n$ \\
\hline Janxery & 3398 & 2500 & 5898 & -49.74 & 450 & -4524 & -1531 & 1426 & 485 & 0.00 \\
\hline Fobnery & 0302 & 25.00 & 8892 & -25.53 & 450 & -21.13 & $-6,46$ & 18.42 & -288 & 0.00 \\
\hline Harch & 114.68 & 2500 & 139.68 & -230 & 450 & 211] & 0.00 & 3759 & -220 & 0.00 \\
\hline Apri & 165.55 & 25.00 & 100.65 & 758 & 450 & 1208 & 0.00 & 55.42 & 0.00 & 0.00 \\
\hline May & 204.93 & 2500 & 229.93 & 40.93 & 450 & 4543 & 0.00 & 8553 & 0.00 & 0.00 \\
\hline Jing & 23426 & 2500 & 25926 & 46.06 & 450 & 5056 & 0.00 & 83.07 & 0.00 & 021 \\
\hline Juty & 24592 & 2500 & 27092 & 6651 & 450 & 71.01 & 0.00 & 10103 & 0.00 & 824 \\
\hline Aluges: & 221.83 & 2500 & 24683 & 54.19 & 450 & 5869 & 0.00 & 87.71 & 0.00 & 821 \\
\hline Saptumbor & 187.79 & 25.00 & $2 t 2.79$ & 21.17 & 450 & 25.67 & 0.00 & 67.15 & 0.00 & 4.18 \\
\hline Octobor & 12685 & 2500 & 151.85 & -236 & 450 & 2.14 & 0.00 & 41.76 & 0.00 & 0.00 \\
\hline Noramber & 251 & 2500 & 88.61 & -2256 & 450 & -18.06 & -652 & 2286 & .238 & 0.00 \\
\hline Decomber & 31.72 & 25.00 & 56.72 & $-4,47$ & 450 & 39.97 & - $\{3.53$ & 13.72 & 4.18 & 0.00 \\
\hline CLOUOY D & Y HEATBALAN & NCE & & & & & & & & \\
\hline Manth & Deapiod Perio & iod $W / h_{2} 2$ & & Unocapod Pe & enodW/m2 & & Mon'hy Pegure & rementkth & Frestifiload & dkWh \\
\hline & PVnotgain & Intonits & Towarl & PV notgin & Intronals & Taenil & Hoaing & Coosing & Hoain & $\cos \cos ^{\circ}$ \\
\hline Jantery & -1524 & 4500 & 29.76 & 5267 & 450 & 4.17 & 260 & 200 & -137 & 0.00 \\
\hline Fobreary & -0.55 & 40.00 & 3945 & $\$ 6,72$ & 450 & $\$ 222$ & -278 & 243 & -0.81 & 0.00 \\
\hline March & $24 A 7$ & 35.00 & 59,47 & -18.09 & 450 & -4439 & -0.94 & $2 \pi$ & -240 & 0.00 \\
\hline Apai & 5935 & 30.00 & 89.35 & 308 & 450 & 0.64 & 0.00 & 298 & 0.00 & 0.00 \\
\hline $\mathrm{M} z \mathrm{y}$ & 8590 & 25.00 & 110.90 & 6.91 & 450 & II:A1 & 0.00 & 235 & 0.00 & 0.00 \\
\hline Juno & 117.63 & 2500 & 14263 & 2205 & 450 & 27.45 & 0.00 & 326 & 0.00 & 0.02 \\
\hline Juty & 129.35 & 25.00 & 15435 & 30.74 & 450 & 3524 & 0.00 & 758 & 0.00 & 1.12 \\
\hline Auges & 121.02 & 25.00 & 146.02 & 2383 & 450 & 2833 & 0.00 & 8.05 & 0.00 & 1.50 \\
\hline Septenber & 10135 & 30.00 & 131.35 & 196 & 450 & 646 & 0.00 & 4211 & 0.00 & 0.46 \\
\hline Octuber & 60.06 & 3500 & 95.06 & -11.79 & 450 & -729 & -0.41 & 380 & 0.00 & 0.00 \\
\hline Nowertbor & 1482 & 40.00 & 54.82 & $-25 \times 0$ & 450 & -21.30 & -125 & 230 & -0.39 & 0.00 \\
\hline Decomber & -857 & 45.00 & 35.43 & 46.4 & 450 & 42.14 & -4.02 & 248 & -1.18 & 0.00 \\
\hline OYERALIE & VERGY REQURA & RENENTS AND & DPROOUCTIOA & & & & & & & \\
\hline Month & Coding Consun & umpion & Hoating Consen & motion & Fans & Lighting and $t$ & & & & \\
\hline & 1 koop & KWW Eectic & 11000 & Wh Eectic & kWh & Power kWh & consemod & & & \\
\hline January & 0.00 & 0.00 & 022 & 581 & 19.75 & 4.00 & 2856 & & & \\
\hline Fabneary & 028 & 624 & 022 & 287 & 1784 & 3.30 & 3026 & & & \\
\hline March & 029 & 11.53 & 022 & 081 & 18.75 & 3.10 & 35.18 & & & \\
\hline Apri & 028 & 16.68 & 022 & 0.00 & 19.11 & 2.72 & 3851 & & & \\
\hline Nay & 028 & 25.11 & 022 & 0.00 & 19.75 & 2.64 & $47 / 4$ & & & \\
\hline لsino & 029 & 27.60 & 022 & 0.00 & 19.11 & 255 & 4926 & & & \\
\hline Juty & 029 & 33.70 & 022 & 0.00 & 19.75 & 2.64 & 56.09 & & & \\
\hline Auguse & 028 & 30.42 & 0.22 & 0.00 & 19.75 & 264 & 5280 & & & \\
\hline Soptonber & 029 & 21.72 & 022 & 0.00 & 19.11 & 2.70 & 4353 & & & \\
\hline Oatoor & 029 & 1303 & 022 & 0.09 & 19.75 & 3.04 & 3500 & & & \\
\hline Novernbor & 029 & 7.19 & 022 & 234 & 19.19 & 3.18 & 31.82 & & & \\
\hline Deconbex & 0.00 & 0.00 & 022 & 5.10 & 19.75 & 4.00 & 2884 & & & \\
\hline TOTALM2 & & 19322 & & 17.02 & 23251 & 36.49 & 47924 & & & \\
\hline
\end{tabular}




\begin{tabular}{|c|c|c|c|c|c|c|c|}
\hline EI LET & $8 E t$ & $912 L 1$ & 110 & & $8+20$ & & $24 \times 101$ \\
\hline 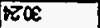 & 1885 & 90"St & 1000 & $\bar{Z} 0$ & 201 & $\mathscr{\infty} 0$ & 29110000 \\
\hline$\alpha \propto \infty$ & $100 t$ & $95+6$ & $\{000$ & $Z 0$ & 9001 & $\notin 0$ & squason \\
\hline $1 Z x$ & एా & sost & 1000 & $\approx 0$ & $t+4 i$ & $\mathbb{E} 0$ & 10900 \\
\hline |દ'\& & $\angle I \varepsilon$ & $155+1$ & 1000 & $\approx 0$ & for 2 & 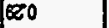 & mquados: \\
\hline $15 t r$ & 192 & gost & 100 & $2 z 0$ & 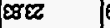 & $6 Z 0$ & isnfory \\
\hline 96 it & tor & gost & $1 \infty 0$ & $\pi c$ & $81^{\circ} \hbar 2$ & $\notin 0$ & ins \\
\hline otos & ser & $95 \mathrm{H}$ & 1000 & $\pi 0$ & lezट & $\Leftrightarrow 20$ & and \\
\hline 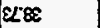 & 192 & sost & $\infty \infty$ & $\mathbb{Z} 0$ & 9012 & $\notin 0$ & KकW \\
\hline 6 & $\angle 6 B 2$ & s5n & $\infty \infty 0$ & $\pi 0$ & $9 r<1$ & $\mathbb{Z O}$ & aty \\
\hline $68 \%$ & Es\& & gosi & 1000 & $\pi 0$ & $1 E+1$ & 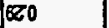 & पPEF| \\
\hline$E R$ & $\cos$ & (05\&l & 1000 & $\approx 0$ & $\mathrm{EOt}$ & $\theta 80$ & Menxpey \\
\hline SOQ & 185t & grost & w0 & $\pi 0$ & IE6 & $\otimes 0$ & frankef \\
\hline prounsus & 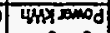 & पथन्म & 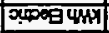 & ठक्वा & 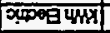 & क्णा & \\
\hline $44 \times 1201$ & pue cupugn & sere & 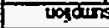 & 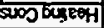 & Uogdurs & जuक & Tue \\
\hline
\end{tabular}

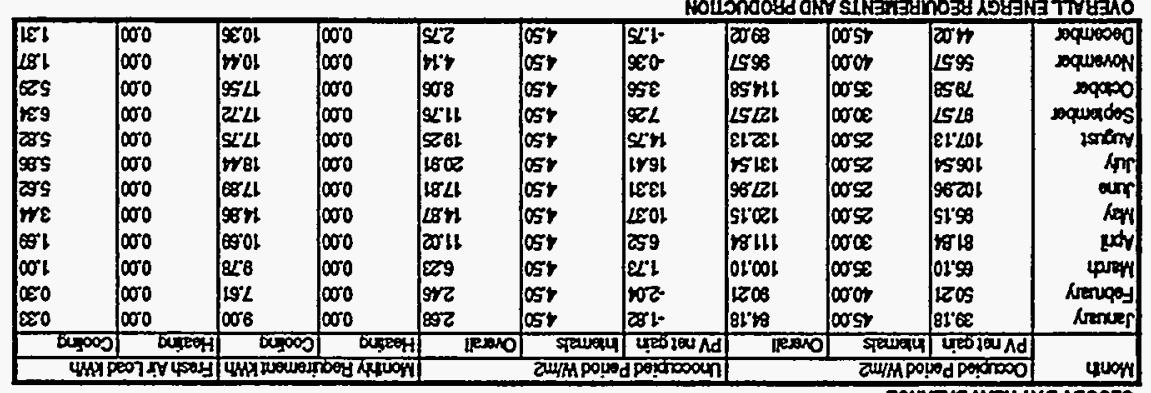

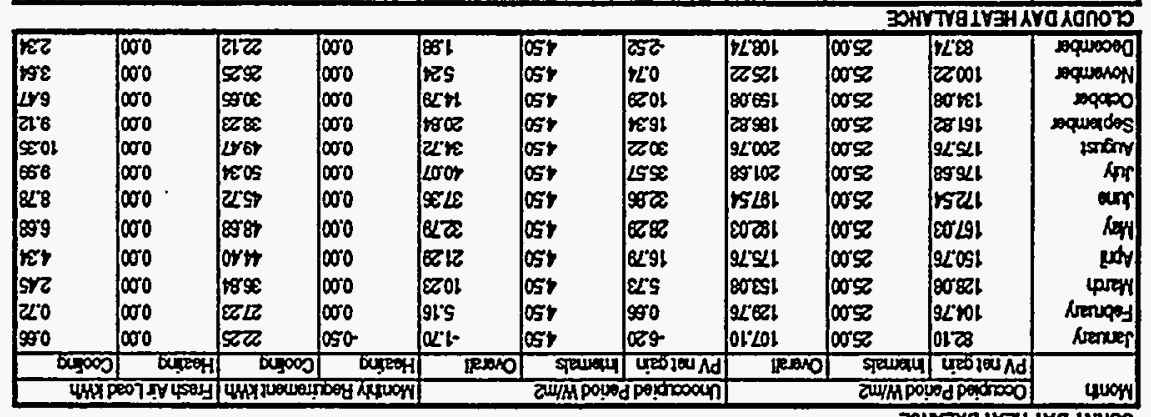

\begin{tabular}{|c|c|c|c|c|c|c|c|c|c|c|}
\hline & & & & & & & & & 81VE & Va dinting \\
\hline 9015 & FSES & Tith & 1861 & 1966 & 105816 & mas & 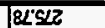 & 1081 & $\mathscr{x} \mathscr{E}$ & Doqueser \\
\hline 2018 & $\infty<s$ & $\infty \approx 01$ & os 61 & 10521 & øLE! & ste & 6rols & 10602 & $\infty \pi$ & squenow \\
\hline 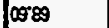 & 8620 & s58b & $90<6$ & $\varepsilon x$ & 8921 & |16st & 25848 & ark & $18 \pi$ & $\max$ \\
\hline 8552 & $x+2$ & lezt & $|\alpha<b|$ & 608 & 98086 & 10020 & $18 m$ & 1009 & $\mathscr{Z} \boldsymbol{z}$ & maluados \\
\hline 뚜로 & loste & $91 \div 36$ & trot & $\& \Delta$ & 108600 & 55001 & Ærtor & 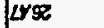 & 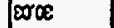 & $\ln 6$ \\
\hline $\cos 2$ & SOLL & 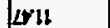 & 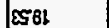 & $E Z \in S$ & oor LIZ & LISSL & 926 & nes & $\infty \approx$ & nos \\
\hline कo & $\operatorname{arcz}$ & $\alpha \cdot u$ & loz81 & $\cos \theta$ & | $6 z 512$ & CSLLL & L800st & $10^{\circ} \boldsymbol{Z}$ & $01: 62$ & an \\
\hline$\varnothing \otimes$ & arr & $\$ 501$ & groe & ars & 0002 & [ะ91 & soLOs & $\varepsilon r x$ & $\operatorname{los} 26$ & \\
\hline 178 & $\circledast<5$ & 0018 & $\omega^{\prime}$ L & $\angle s 9$ & 99012 & $51 \% 206$ & osest & erLIZ & 228 & \\
\hline 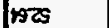 & LIZES & 058 & 1002 & $\alpha \theta$ & 9596 & $81 \cdot 0$ & 985 & 1581 & $100 \%$ & prot \\
\hline$\approx z 6 r$ & act & 218 & $\$ 65$ & aroz & 28थ1 & $\infty$ & soers & St'Bt & arza & Nenupey \\
\hline Est & $006 t$ & $E 01$ & $\psi \omega$ & $\cos n$ & $\angle S S \mid$ & $\infty \approx$ & $5 s 908$ & 854 & est & frerier \\
\hline petcooch & peqpon & Tprop & hans & pord & pegcison & percroon & peqdoso & pexposoun & penta & \\
\hline
\end{tabular}

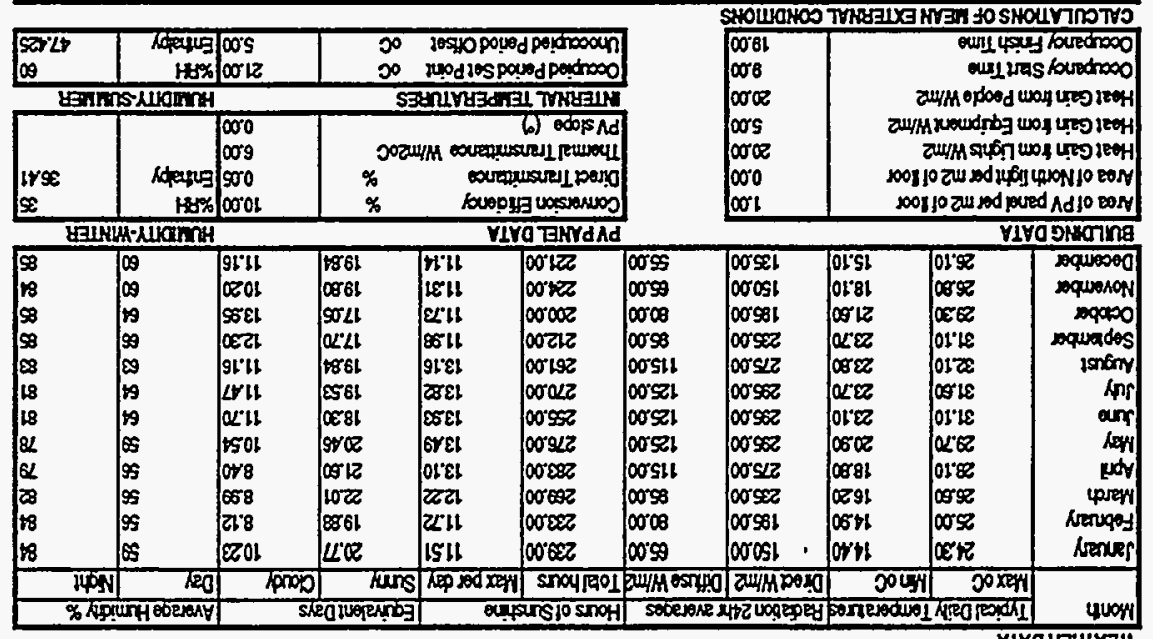

\begin{tabular}{|c|c|c|c|c|c|c|c|}
\hline & & & & & & \multicolumn{2}{|c|}{ YLOEZHIVבM } \\
\hline & & $\begin{array}{l}01 \% 1 \\
050\end{array}$ & 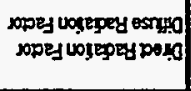 & $\begin{array}{l}\Psi \infty O Z \\
M \angle \infty \\
N O S E\end{array}$ & $\begin{array}{l}\text { eprey } \\
\text { eprye] } \\
\text { oprowor }\end{array}$ & $\begin{array}{r}\text { [eopostans } \\
\text { uresin }\end{array}$ & $\begin{array}{l}\text { aews } \\
\text { 40 }\end{array}$ \\
\hline
\end{tabular}


OPTION 1: SEMLTRANSPARENT SINGLE-GLAZED PV ROOF

STEDATA

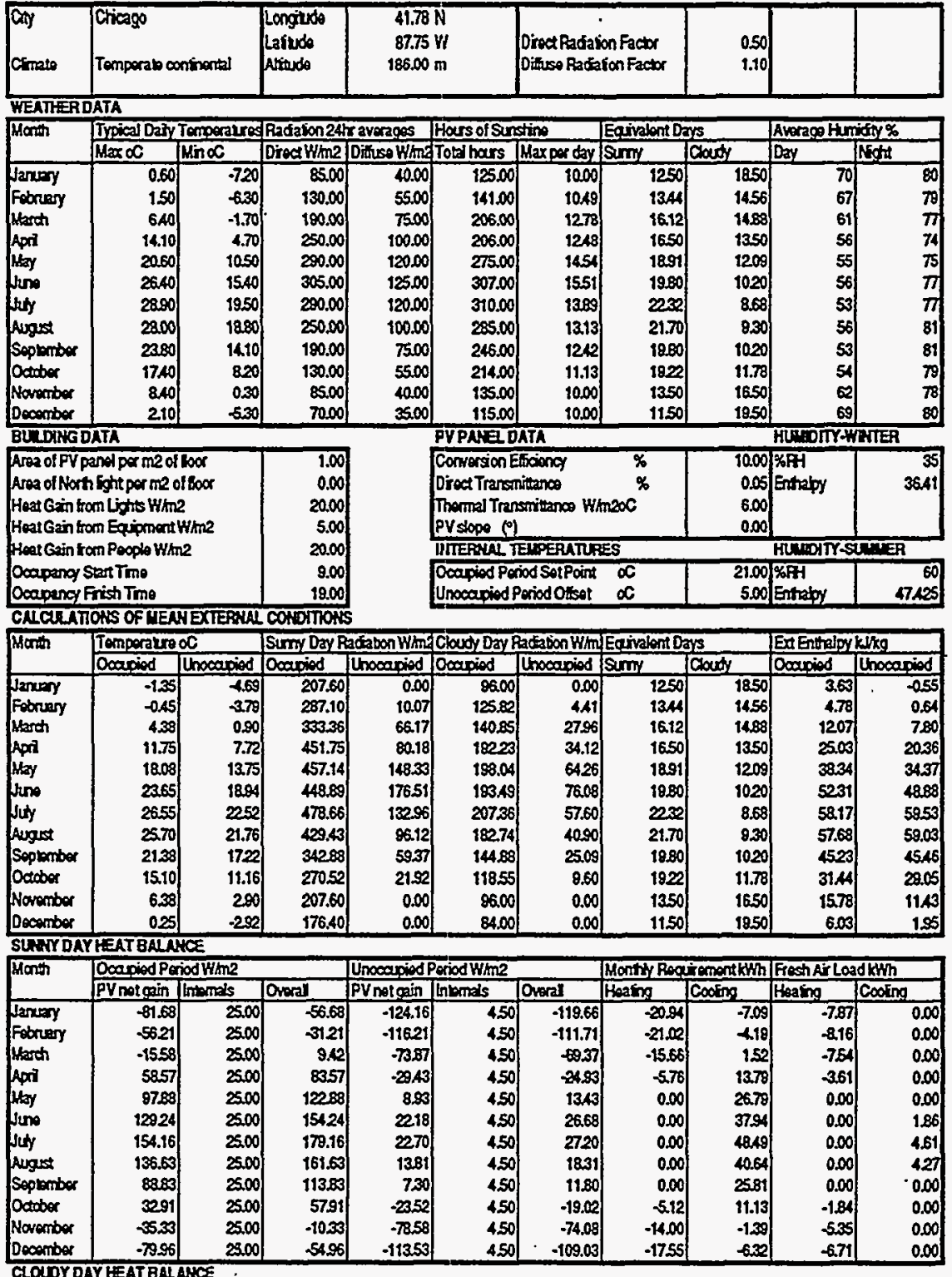

CLOWO Y OAY HEAT BNLANCE

\begin{tabular}{|c|c|c|c|c|c|c|c|c|c|c|}
\hline \multirow[t]{2}{*}{ Manth } & \multicolumn{3}{|c|}{ Ocapiod PeriodWhnz } & \multicolumn{3}{|c|}{ Unocapied Period Whan } & \multicolumn{4}{|c|}{ Monthy RegirementKWh Frosh Al LosdKWh } \\
\hline & PVnetgain & hrengals & Owaral & PVnotenin & lontermis & Oworal & Hoaing & $\sqrt{\cos \sin \theta}$ & Heaing & cooing \\
\hline Janury & -109.86 & 45.00 & -5486 & -124.16 & 450 & -119.66 & 30.99 & -12.00 & -11.64 & 0,0 \\
\hline Fabruary &.$\$ 9.93$ & 40.00 & $\$ 6.93$ & -117.64 & 450 & -113.14 & -23.06 & 220 & 8.84 & 000 \\
\hline Narch & -64.18 & 35,00 & -20.18 & -8352 & 450 & -79.02 & -1646 & 434 & 698 & 0.00 \\
\hline Apr & -696 & 30,00 & 2304 & $-41,06$ & 450 & $\$ 56$ & 691 & 3.81 & -285 & 0.00 \\
\hline Way & 3245 & 2500 & 57.45 & 270 & 4.50 & 720 & 0.00 & 8.17 & 0.00 & 0.00 \\
\hline huno & 64.76 & 25.00 & 89.76 & 9.82 & 4.50 & 1432 & 0.00 & 1120 & 0.00 & 0.9 \\
\hline Wuly & 85.66 & 25.00 & 110.66 & 8.67 & 450 & 13.17 & 0.00 & 1121 & 0.00 & $1 . \pi$ \\
\hline August & 7434 & 2500 & 9934 & 487 & 450 & 9.37 & 0.00 & 10.46 & 0.00 & 1.8 \\
\hline Seopember & 3283 & 30.00 & 68.83 & $-1,36$ & 4.50 & 3.14 & 0.00 & 7A7 & 0.00 & 0.00 \\
\hline foctosex & 5.47 & 3500 & 29.53 & -26.63 & 450 & -2213 & -365 & 3.48 & -1.13 & 0.00 \\
\hline Norember & -63.51 & 40.00 & -2351 & -7858 & 4.50 & -74.08 & -17.11 & 2388 & 654 & 0.0 \\
\hline Docombar & -10329 & 4500 & -5829 & -11353 & 4.50 & -109.03 & -29.76 & -11.37 & $-11,38$ & 0.00 \\
\hline
\end{tabular}

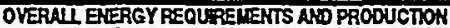

\begin{tabular}{|c|c|c|c|c|c|c|c|}
\hline \multirow[t]{2}{*}{ Mon'th } & \multicolumn{2}{|c|}{ Coding Consempion } & \multicolumn{2}{|c|}{ Hoating Consumpion } & \multirow{2}{*}{$\begin{array}{l}\text { Fars } \\
\text { xthh }\end{array}$} & \multirow{2}{*}{$\begin{array}{l}\text { Lighing and } \\
\text { Powor Kthth }\end{array}$} & \multirow{2}{*}{$\begin{array}{l}\text { Total kth } \\
\text { Consemod }\end{array}$} \\
\hline & $1 / 000$ & $\mathrm{kWh}$ Bectic & 1 coop & $\mathrm{kWm}$ Bestic & & & \\
\hline lanery & 0.00 & 0.00 & 022 & 1588 & 11.75 & 6.34 & 3396 \\
\hline ebrasy & 0.00 & 0.00 & 022 & 1358 & 10.61 & 456 & 28.75 \\
\hline tarch & 0.00 & 0.00 & 022 & 1036 & .11 .75 & 4.12 & 2623 \\
\hline adi & 0.00 & 0.00 & 0.22 & 427 & 11.37 & 323 & 1887 \\
\hline tey & 028 & 9.99 & 022 & 0.00 & 11.75 & 264 & 2437 \\
\hline uno & 029 & 1484 & 022 & 0.00 & 11.37 & 255 & 2876 \\
\hline lutj & 029 & 1888 & 022 & 0.00 & 11.75 & 264 & 3327 \\
\hline ugat & 028 & 1634 & 022 & 0.00 & 11.75 & 264 & 30.73 \\
\hline oppentor & 020 & 9.51 & 022 & 0.00 & 1137 & 3.05 & 2304 \\
\hline & 020 & 4.17 & 022 & 2.61 & 11.75 & 388 & 2234 \\
\hline nbor & 0.00 & 0.00 & 0.22 & 955 & 11.37 & 5.03 & 2595 \\
\hline locember & 0.00 & 0.00 & 022 & $: 53$ & 11.75 & 6.54 & 3282 \\
\hline TOTALm2 & & 73.74 & & 70.78 & 138.33 & $\overline{47.14}$ & 329.98 \\
\hline
\end{tabular}




\section{OPTION 1: SEMKTRANSPARENT SINGLE-GLAZED PV ROOF}

\begin{tabular}{|c|c|c|c|c|c|c|c|c|c|c|}
\hline \multirow{4}{*}{\begin{tabular}{|l|} 
ang \\
Chrato \\
HEATHERD \\
Manth
\end{tabular}} & \multicolumn{2}{|c|}{$\begin{array}{l}\text { Cincinai } \\
\text { Tonperab oconinental }\end{array}$} & 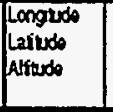 & $\begin{array}{r}39.15 ! \\
84.52 \\
23200\end{array}$ & & $\begin{array}{l}\text { Droct Radaic } \\
\text { Dimuso Padei }\end{array}$ & $\begin{array}{l}\text { ion Factor } \\
\text { ion Factor }\end{array}$ & $\begin{array}{l}0.50 \\
1.10\end{array}$ & & \\
\hline & DATA & & & & & & & & & \\
\hline & Typical Daty & Temoerayes & Bradalon 24h & rarocogos & Hors of Suns & $\operatorname{sing}$ & Equivelont of & & Anoreos $\mathrm{Hum}$ & ifiv\% \\
\hline & $M \times \infty$ & Hinoc & Direct H/m2 & Dimuso W/mat & Tog hars II & Iflaxporday & Sumy & Cooudy & Day & Nont \\
\hline Jantary & 620 & 330 & 8500 & 40.00 & 108.00 & 10.00 & 10.80 & 2020 & 68 & 8 \\
\hline Fobruery & 6.30 & -200 & 130.00 & 55.00 & 129.00 & 1024 & 1260 & 15.0 & 64 & 81 \\
\hline Nerch & 11.10 & 0.70 & 190.00 & 7500 & 173.00 & 11.16 & 1550 & 1550 & 57 & 79 \\
\hline Apri & 1800 & 6.60 & 250.00 & 100.00 & 201.00 & 11.96 & 1680 & 1320 & 53 & 76 \\
\hline Nixy & 2380 & 11,30 & 290.00 & 12000 & 241.00 & 1274 & 18991 & 1200 & 53 & 78 \\
\hline fine & 2800 & 1720 & 305.00 & 125.00 & 30500 & 14.75 & 20.70 & 830 & 55 & 80 \\
\hline Jusy & 30500 & 19.10 & 290000 & 120.00 & 32200 & $14 / 4$ & 2232 & 8.58 & 52 & 82 \\
\hline Aungust & 3020 & 1830 & 250.00 & 100.00 & 291.00 & 13.41 & 21.70 & 930 & 52 & 86 \\
\hline Septanber & 2680 & 1420 & 190.00 & 7500 & 254.00 & 1245 & 20,40 & 8.50] & so & 86 \\
\hline patiber & 2050 & 820 & $\{30.00$ & 5500 & 21300 & 1126 & 1891 & 1209 & 61 & 85 \\
\hline Noventbor & 1180 & 220 & 8500 & 40.00 & 145.00 & 10.51 & 1380 & 1620 & 60 & 81 \\
\hline Docombor & 5,00 & -230 & 7000 & 3500 & 126.00 & 10.16 & 1240 & 18.50 & $\Leftrightarrow$ & 82 \\
\hline BLLIMG & JATA & & & & PVPAFELD & DATA & & & HUDOTYW & NTIEA \\
\hline \begin{tabular}{|l|} 
Arez of PV \\
Arez offNor
\end{tabular} & $\begin{array}{l}\text { pand per m2 of } \\
\text { nifhiper m20 }\end{array}$ & $\begin{array}{l}\text { ibox } \\
\text { oflibox }\end{array}$ & $\begin{array}{l}1.00 \\
0.00\end{array}$ & & $\begin{array}{l}\text { Conversion E: } \\
\text { Direct Transinn }\end{array}$ & Eisioncy & & $\begin{array}{r}10.00 \\
0.05\end{array}$ & For & 35 \\
\hline $\begin{array}{l}\text { Heat Gain } \\
\text { HoatGain }\end{array}$ & on Lights Whim & & $\begin{array}{r}20.00 \\
5.00\end{array}$ & & $\begin{array}{l}\text { Thermal Trans } \\
\text { PV siope (") }\end{array}$ & instivanos Wh & $\max x^{\circ}$ & $\begin{array}{l}6.00 \\
0.00\end{array}$ & & \\
\hline Hoat Gaint & om Pesplo H/m & & 20.00 & & INTERYLL TE & EPPEPATUR: & & & HUDWT-SI & ITER \\
\hline $\begin{array}{l}\text { Ooapangy } \\
\text { Ocompang }\end{array}$ & $\begin{array}{l}\text { SunTmo } \\
\text { Frishtme }\end{array}$ & & $\begin{array}{r}900 \\
1800\end{array}$ & & $\begin{array}{l}\text { Doapiod Peri } \\
\text { Unowinis P }\end{array}$ & riod SotPcoint & $\begin{array}{l}\alpha \\
\alpha\end{array}$ & $\begin{array}{r}21.00 \\
500\end{array}$ & EF+1 & $\begin{array}{r}60 \\
4705\end{array}$ \\
\hline CNICUAI & IONS OFWEN & NEXTERMI & LCOHDITOHS & & & & & & & \\
\hline Monts & Tremperature & & Surm Day $\mathrm{A}^{2}$ & Hon W/mIC & Cloudy Day $R$ & Raceon W/m & Equatorit of & & JErEntralor! & $\sqrt{k \sqrt{x g} g}$ \\
\hline & Oocapiod & throcapiod & ocoupiod & Uhooupied & Ocapiod II & thoosapied & $\sin y$ & Coovery & Dowoind & thocapiod \\
\hline Jaxemy & 3.83 & .025 & 207.60 & 0.00 & 86.00 & 0.00 & 1080 & 2020 & 11.96 & 6.36 \\
\hline Fabruery & 4.00 & 0.06 & 20420 & 5.00 & 128.83 & 219 & 1260 & 1510 & 11.79 & 6.71 \\
\hline March & 8.50 & 4.04 & 381.68 & 31.66 & 16127 & 1338 & 1550 & 1550 & 18.91 & 1369 \\
\hline Apri & 15.15 & 1026 & 471/10 & 66.14 & 200.50 & 28.14 & 1680 & 1320 & 3124 & 2631 \\
\hline intay & 20.83 & 15.73 & 521.63 & 10226 & 225.98 & 4430 & 1891 & .1209 & 4393 & 4035 \\
\hline fise & 2590 & 20.93 & 47237 & 159.74 & 203.61 & 6885 & 20.70 & 930 & 57.64 & 56.01 \\
\hline Jichy & 27.88 & 2286 & 460:82 & 145.70 & 199.53 & @.12 & 2232 & 8.58 & 61.04 & 6303 \\
\hline August & 2723 & 2213 & 42058 & 10244 & 178.97 & 4350 & 21.70 & 830 & 59.33 & 6255 \\
\hline Soptember & 2365 & 1825 & 36.14 & 59.90 & 1457 & 2531 & 20,40 & 8.60 & 4924 & 5029 \\
\hline Octcorer & 17A3] & 1215 & 26740 & 24.14 & 117.19 & 10.58 & 1891 & 1209 & 3998 & 3294 \\
\hline November & 9.40 & 529 & 19758 & 7.16 & 9137 & 331 & 1380 & 1620 & 2121 & 1641 \\
\hline Decomber & 3.85 & 0.34 & 173.60 & 200 & 8267 & 0.55 & 1240 & 18.60 & 1200 & 727 \\
\hline SURAYOA & YHEATBMLA & & & & & & & & & \\
\hline Mant & Doogodpen & rod W/m2 & & $\operatorname{apiod} P$ e & Period W/m2 & & Ionthy Roa & kKWh! & Aflas & ad \\
\hline & PVnotgain & Insmats & averal & PV notgain & Intomels & Thoral & Heating & $\mathrm{mg}$ & Hoating & Cooting \\
\hline Faxery & 50.53 & 2500 & -25.63 & -9748 & 450 & .5288 & -14.06 & $.2 \pi$ & 5.07 & 0.00 \\
\hline Fobruary & -27.72 & 2500 & -272 & -0439 & 4.50 & -80.80 & -15.86 & -0.34 & -5.96 & 0.00 \\
\hline March & 2137 & 2500 & 6637 & -6.75 & 450 & $\$ 925$ & -1286 & 7.19 & 521 & 0.00 \\
\hline Apti & 83.23 & 25.00 & 108.83 & • 17.71 & 450 & -1321 & 311 & 1830 & -1.57 & 0.00 \\
\hline Hay & 130.66 & 2500 & 155.66 & 9.17 & 450 & 13.67 & 0.00 & 3306 & 0.00 & 0.00 \\
\hline Jino & 148.67 & 2500 & 173.67 & 199?] & 450 & 24.41 & 0.00 & 4300 & 0.00 & 4.06 \\
\hline fury & 157.61 & 25.00 & 18261 & 179 & 450 & 2215 & 0.00 & 47.7 & 0.00 & 5.83 \\
\hline Aevgest & 14355 & 2500 & 168.55 & 1202 & 450] & 17.12 & 0.00 & $41 . \pi$ & 0.00 & 4.96 \\
\hline Soptantibor & 10228 & 2500 & 12729 & 4.62 & 450 & 9.12 & 0.00 & 2857 & 0.00 & 0.71 \\
\hline Odicbor & 46.07 & 2500 & 71.07 & -16.98 & 450 & $\mid 12 A B$ & -330 & 134 & 0.00 & 0.0 \\
\hline Noventiber & - 19.71 & 2500 & 520 & -248 & 450 & .5798 & -1120 & 0.73 & -1.03 & 0.00 \\
\hline Doconber & -59.07 & 25.00 & -34.07 & -8348 & 450 & -83.88 & $-15,45$ & +22 & 5.81 & 0.00 \\
\hline CLOWYD & AYKEAT BALL & ANCE & & & & & & & & \\
\hline Mants & Deapiod $P$ an & codW/m2 & & $\mathrm{dP}$ & W/m2 & & Montry $R \circ a$ & mathon & Ifrostifir Loa & $d x w h$ \\
\hline & PVnatgain & In $x$ mads & Oweral & PVnat gain & Inemals & Doral & Heating & $\operatorname{cosing}$ & Hoasing & Coofing \\
\hline$\sqrt{2 x u x y}$ & .7821 & 45.00 & -23381 & -97.48 & 450 & .82 .88 & -2628 & -683 & $-9.4 B$ & 0.00 \\
\hline Fobruery & $-\infty A 5$ & 40.00 & $-20,45$ & -86.10 & 450 & -90.60 & -19.53 & +53 & .728 & 0.00 \\
\hline March & -3428 & 3500 & 0.72 & $\$ 37$ & 450 & $-\infty .37$ & -13.86 & 0.11 & -521 & 0.00 \\
\hline Apr & 1555 & 30.00 & 45.55 & -2731 & 450 & .2281 & 421 & 6.01 & -131 & 0.00 \\
\hline Nay & 56.01 & 25.00 & 81.01 & 5.46 & 450 & -0.86 & -0.16 & 8.79 & 0.00 & 0.00 \\
\hline Jine & 80:8t & 25.00 & 105:81 & 1096 & 450 & 15.46 & 0.00 & 11.85 & 0.00 & 182 \\
\hline Jubly & 92.66 & 2500 & 116.56 & 12.10 & 450 & 16.60 & 0.00 & 1214 & 0.00 & 227 \\
\hline August & 8254 & 25.00 & 107.5A & 7.76 & 450 & 1226 & 0.00 & 11.50 & 0.00 & 213 \\
\hline Sopternber & 5240 & 30.00 & 8240 & -4.11 & 450 & 0.39 & 0.00 & 796 & 0.00 & 0.33 \\
\hline october & 8.14 & 35.00 & 43.14 & $-20,41$ & 450 & . 15.91$]$ & -269 & 522 & 0.00 & 0.00 \\
\hline Novenber & -46.53 & 40.00 & -6.53 & $-\infty .45$ & 450 & .58 .25 & -13.37 & -1.06 & 4.73 & 0.00 \\
\hline Decomber & -82.03 & 45.00 & -37.03 & -83.75 & 450 & -25 & .2324 & -689 & -8.72 & 0.00 \\
\hline OVERALI & XERGYREQU & MREMENTSA & MDPRODUC & गुण & & & & & & \\
\hline Month & Coding Cons & impion & Heating Cons & empion & Fans & Lighing and & kwn & & & \\
\hline & $10 \cos$ & LWh Gactic & 1000 & tesm Eoctic & tem & Powerksh & Consumed & & & \\
\hline Janeryy & 0.00 & 0.00 & 022 & 1220 & 1323 & 6.68 & 3210 & & & \\
\hline Fobruary & 0.00 & 0.00 & 0.22 & 1081 & 11.55 & 4.69 & 27.44 & & & \\
\hline Narch & 0.00 & 0.00 & 022 & 825 & 1323 & 4.19 & 25.67 & & & \\
\hline 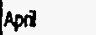 & 0.29 & 6.25 & 022 & 229 & 1280 & 321 & 2525 & & & \\
\hline 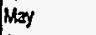 & 029 & 1224 & 022 & 0.04 & 1323 & 264 & 28.14 & & & \\
\hline bino & 028 & 1736 & 0.22 & 0.00 & 1280 & 255 & 3271 & & & \\
\hline Juty & 029 & 19.43 & 0.22 & 0.00 & 1323 & 2.64 & 3530 & & & \\
\hline August & 029 & 1727 & 022 & 0.00 & 1323 & 2.64 & 33.14 & & & \\
\hline Soptember & 029 & 10.74 & 022 & 0.00 & 1280 & 3.03 & 2657 & & & \\
\hline Detober & 029 & 533 & 0.22 & 133 & 1323 & 3.84 & 22.73 & & & \\
\hline lovenber & 0.0 & 0.00 & 0.22 & 7.44 & 1280 & 458 & 25.19 & & & \\
\hline Docombor & 0.00 & 0.00 & 0.22 & 83 - & 1323 & 636 & अ,A1 & & & \\
\hline TOTAL/m2 & & 8922 & & 1.15 & 155.75 & 47.42 & 346.65 & & & \\
\hline
\end{tabular}


STEDATA

\begin{tabular}{|c|c|c|c|c|c|c|c|c|c|c|}
\hline \multirow{2}{*}{\begin{tabular}{|l|} 
aty \\
camato \\
WEATREAD
\end{tabular}} & \multicolumn{2}{|l|}{$\begin{array}{l}\text { San Franciseos } \\
\text { Temporat cost }\end{array}$} & $\begin{array}{l}\text { Longrude } \\
\text { Lafudo } \\
\text { Afitude }\end{array}$ & \multicolumn{2}{|c|}{$\begin{array}{r}37.62 \mathrm{H} \\
122.38 \mathrm{ly} \\
2.00 \mathrm{~m}\end{array}$} & \multicolumn{2}{|c|}{$\begin{array}{l}\text { Oiroct Padaion Factox } \\
\text { Difiso Padiation Facto }\end{array}$} & $\begin{array}{l}0.50 \\
1.10\end{array}$ & & \\
\hline & & & & & & & & & & \\
\hline \multirow[t]{2}{*}{ Manth } & \multicolumn{2}{|c|}{ Irpical Daily Tomperatures } & \multicolumn{2}{|c|}{ Radaion 24 tr averages } & \multicolumn{2}{|c|}{ Hours of Sunting } & \multicolumn{2}{|c|}{ Egivalentoays } & \multicolumn{2}{|c|}{ Avoreg Humiogy } \\
\hline & $\operatorname{Max} \infty$ & Mnoc & Drectw/m2 & Dilinso W/in2 & Totihars & Max pox day & Sum & Coudy & Day & Nigt \\
\hline Jamay & 1300 & 5.40 & 8500 & 40.00 & 15400 & 10.00 & 1510 & 15.60 & 75 & 85 \\
\hline Fobruary & 1470 & 6.30 & 130.00 & 5500 & 191.00 & 1156 & 16.52 & 1148 & 71 & 85 \\
\hline March & 1640 & 720 & 190.00 & 75.00 & 20200 & 1243 & 2108 & 9.22 & 66 & 83 \\
\hline Apri & 1790 & 8.40 & 250.00 & 100.00 & 297.00 & 13.94 & 2130 & 8.70 & 66 & 85 \\
\hline Hay & 19.40 & 9.80 & 290.00 & 120.00 & 328.00 & 1490 & 2201 & 8.99 & 66 & $\notin$ \\
\hline fins & 2120 & 1130 & 305.00 & 125.00 & 341.00 & 1535 & 2220 & 780 & 65 & 87 \\
\hline foty & 2220 & 11.90 & 250.00 & 120.00 & 295.00 & 1420 & 20.77 & 1023 & 69 & 90 \\
\hline August & 2210 & 1210 & 250.00 & 100.00 & 275.00 & 13.65 & 20.15 & 1085 & 69 & 90 \\
\hline Septriber & 2320 & 1220 & 190.00 & 75.00 & 275.00 & 12.73 & 21.60 & 8.40 & 66 & 88 \\
\hline oatcor & 21.40 & 10.30 & 130.00 & 55.00 & 24200 & 11.31 & 2139 & $8.5 \mathrm{t}$ & 65 & 86 \\
\hline Norember & 1780 & 7.70 & 85.00 & 40.00 & 198.00 & 10.00 & 1980 & 1020 & 68 & 84 \\
\hline Docomber & 14.00 & 6.10 & 70.00 & 35.00 & 179.00 & 10.31 & 1736 & 1364 & 74 & 86 \\
\hline \multicolumn{4}{|c|}{ BUTOIKGOATA } & & \multicolumn{4}{|c|}{ PVPAIELATA } & \multicolumn{2}{|c|}{ HUT:DIYFWWTER } \\
\hline \multirow{5}{*}{\multicolumn{3}{|c|}{ 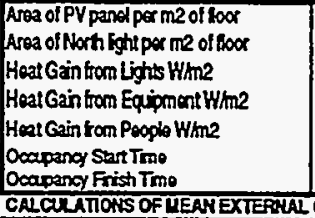 }} & $\begin{array}{l}1.00 \\
0.00\end{array}$ & & \multirow{2}{*}{\multicolumn{3}{|c|}{ 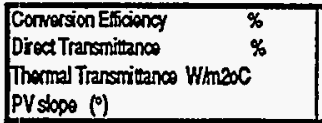 }} & $\begin{array}{r}10.00 \\
0.03\end{array}$ & Extaby & $\begin{array}{r}35 \\
641\end{array}$ \\
\hline & & & $\begin{array}{r}20.00 \\
5.00\end{array}$ & & & & & $\begin{array}{l}2.00 \\
0.00\end{array}$ & & \\
\hline & & & 20.00 & & IVTERHAL TE & ELPERATURE & & & HUTDITSS & JUUER \\
\hline & & & $\begin{array}{r}9.00 \\
18.00 \\
\end{array}$ & & $\begin{array}{l}\text { Doapiod Pon } \\
\text { Unocapiod P }\end{array}$ & $\begin{array}{l}\text { nod Sol Point } \\
\text { Period Otsot }\end{array}$ & $\begin{array}{l}\infty \\
\infty\end{array}$ & $\begin{array}{r}21.00 \\
5.00\end{array}$ & $\begin{array}{l}\text { \%or } \\
\text { Entraby }\end{array}$ & $\begin{array}{r}60 \\
47425 \\
\end{array}$ \\
\hline & & & COHDITINS & & & & & & & \\
\hline Month & Temperatroo & & Surny Day $\mathrm{R}$ & dation W/m2 & Cloudy Day $\mathrm{B}$ & gofion What & Equivant Da & & ExtEnthelgy & alkg \\
\hline & Oocupind & Unocapiod & Docuipod & thocapiad & Oeapiod & Unocapiad & $\sin y$ & Couds & Ocaioiod & Uhocoupiod \\
\hline Fanuey & 11.10 & 7.84 & 207.60 & 0.00 & 9600 & 0.00 & 1540 & 15.60 & 2801 & 2252 \\
\hline Fobruary & 1260 & 8.00 & 260.51 & 29.06 & 114.17 & 1274 & 1652 & 11.48 & 30.55 & 2519 \\
\hline March & 14.10 & 10.16 & 342.5 & 59.46 & 1482 & 25.18 & 21.08 & 9.22 & 3271 & 2753 \\
\hline Apri & 1553 & $11 / 15$ & $404 A 8$ & 113.94 & 172.12 & 4848 & 2130 & 8.70 & 3608 & 31.15 \\
\hline May & 1700 & 1280 & 46.11 & 15621 & 19326 & 67.67 & 2201 & 8.99 & 3959 & 3484 \\
\hline ons & 1873 & 1448 & 453.11 & 173.49 & 155.3i & 74.78 & 2220 & 7.80 & 4367 & 39.69 \\
\hline foty & 19.63 & 1521 & 468.06 & 140.53 & $202 \pi$ & 60.88 & 20.7 & 1023 & 47.52 & 1264 \\
\hline Augest & 19.60 & 1531 & 41326 & 107.67 & 17585 & $45 \mathrm{BO}$ & 20.15 & 10.85 & 4755 & 4284 \\
\hline Septomber & 20.45 & 15.74 & 334.60 & 6528 & 14138 & 2758 & 21.60 & 8.40 & 4859 & 4354 \\
\hline pouthor & 18603 & 1387 & 26623 & 24.98 & 116.57 & 10.95 & 2139 & 8.51 & 4342 & 37.72 \\
\hline Norember & 1528 & 10.95 & 207.50 & 0.00 & 86.00 & 0.00 & 19.80 & 1020 & 36.10 & 29.66 \\
\hline December & 1203 & 8.64] & 17108 & 380 & 81,A7] & 1.81 & 17336 & 13.64 & 29.94 & 24.53 \\
\hline SUNAYDA & YBEAT BALAN & $\overline{V E}$ & & & & & & & & \\
\hline Manth & Ocoloiad Perio & adW/m2 & & Unocapied Pe & IH/n2 & & YRegi & Contkerh & Frosh ArLaz & dkwh \\
\hline & PVnatgin & Intemals & Onoral & PYnotgain & Intemals & Omoral & Hating & $\operatorname{cosing}$ & Healing & Cooing \\
\hline Januay & -230 & 2500 & 2270 & -16.31 & 4.50 & -11.81 & -255 & 350 & -248 & 0.00 \\
\hline Fobruay & 5.16 & 2500 & 30.16 & -11.55 & 4.50 & -7.05 & -1.53 & 498 & -186 & 0.00 \\
\hline Harah & 1509 & 2500 & 40.09 & 6.67 & 450 & -217 & -0.54 & 8A5 & -150 & 0.00 \\
\hline Apri & 2315 & 25.00 & 48.15 & 0.51 & 450 & 5.01 & 0.00 & 11.75 & -0.14 & 0.00 \\
\hline May & 29.61 & 25.00 & 54.61 & 3.61 & 450 & 8.11 & 0.00 & 1452 & 0.00 & 0.00 \\
\hline Jino & 3365 & 25.00 & 5865 & 6.59 & 450 & 11.09 & 0.00 & 1647 & 0.00 & 0.00 \\
\hline Justy & 3671 & 2500 & 61.71 & 5.27 & 450 & 8.77 & 0.00 & 15.66 & 0.00 & 0.08 \\
\hline August & 3204 & 2500 & 57.04 & 271 & 450 & 721 & 0.00 & 1353 & 0.00 & 0.05 \\
\hline Soplanber & 27.11 & 2500 & 5211 & -0.03 & 450 & 4.47 & 0.00 & 1261 & 0.00 & 0.48) \\
\hline Octeber & 17.69 & 2500 & 4269 & -216 & 450 & 234 & 0.00 & 0.83 & 0.00 & 0.00 \\
\hline Norember & 6.05 & 2500 & 31.05 & -10.11 & 450 & -5.61 & -1.55 & 6.15 & -0.12 & 0.00 \\
\hline Decomber & -353 & 25.00 & 21,47 & -1440 & 450] & -9.90 & -241 & 3.78 & -216 & 0.00 \\
\hline
\end{tabular}

EEAT BALAHCE

\begin{tabular}{|c|c|c|c|c|c|c|c|c|c|c|}
\hline \multirow[t]{2}{*}{ Manth } & \multicolumn{3}{|c|}{ Oconiod Period W/m2 } & \multicolumn{3}{|c|}{ Unocapiod Period Wh/m2 } & \multicolumn{4}{|c|}{ 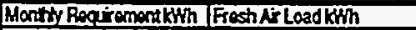 } \\
\hline & PVnolqain & Intone's & Ororal & PV notgain & Intorna's & Overal & Heaing & cosing & Heaing & $\operatorname{cosin} \theta$ \\
\hline Jaxtay & -11.71 & 45.00 & 3329 & -1631 & 450 & -11.81 & .258 & 5.19 & -252 & $\overline{0.00}$ \\
\hline Fobruay & -7.18 & 40.00 & 3282 & -1298 & 450 & -843 & -135 & 3.77 & -120 & 0.00 \\
\hline March & -1.59 & 3500 & 3341 & -957 & 450 & -507 & -0.70 & 3.31 & -0.71 & 0.00 \\
\hline Apti & 356 & 30,00 & 3356 & 5.01 & 450 & -0.51 & -0.06 & 292 & -0.06 & 0.00 \\
\hline Hey & 820 & 25.00 & 3329 & -0.52 & 450 & 398 & 0.00 & 3.49 & 0.00 & 0.00 \\
\hline tho & 11.91 & 25.00 & 36.91 & 327 & 4.50 & 7.77 & 0.00 & 3.73 & 0.00 & 0.00 \\
\hline Wuy & 1434 & 2500 & 39.34 & 355 & 450 & 8.05 & 0.00 & 5.18 & 0.00 & 0.04 \\
\hline August & 1202 & 2500 & 37.02 & 249 & 450 & 6.99 & 0.00 & 5.08 & 0.00 & 0.03 \\
\hline Sepromber & 10.82 & 30.00 & 40.82 & 1800 & 450 & 6.30 & 0.00 & 4.57 & 0.00 & 0.19 \\
\hline October & 5.09 & 3500 & 40.09 & 3.34 & 450 & 1.16 & 0.00 & 4.01 & 0.00 & 0.00 \\
\hline Noromber & 336 & 40.00 & 36.64 & -10.11 & 450 & -5.61 & -0.80 & 3.74 & -0.06 & 0.00 \\
\hline Decentiber & -11.08 & 45.00 & 33.92 & -14.57 & 450 & -10.07 & -1.82 & 4.63 & -1.69 & 0.00 \\
\hline
\end{tabular}

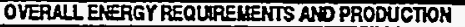

\begin{tabular}{|c|c|c|c|c|c|c|c|}
\hline \multirow[t]{2}{*}{ Hanth } & \multicolumn{2}{|c|}{ Cooling Consumpion } & \multicolumn{2}{|c|}{ Heating Consumption } & \multirow{2}{*}{$\begin{array}{l}\text { Fons } \\
\text { kilh }\end{array}$} & \multirow{2}{*}{$\begin{array}{l}\text { Lighting and } \\
\text { Powor bys }\end{array}$} & \multirow{2}{*}{$\begin{array}{l}\text { Tow kinh } \\
\text { Consumod }\end{array}$} \\
\hline & $1 / 000$ & Ken Bectic & 1000 & kum Eectic & & & \\
\hline Januey & 0.00 & 0.00 & 022 & 225 & $\overline{447}$ & 5.76 & 1247 \\
\hline Fabriay & 0.00 & 0.00 & 022 & 1.36 & 4.03 & 4.10 & 9.50 \\
\hline March & 0.00 & 0.00 & 022 & 0.78 & 447 & 3.63 & 8.88 \\
\hline Apत? & 029 & 4.19 & 022 & 0.06 & 432 & 299 & 1156 \\
\hline May & 020 & 5.15 & 022 & 0.00 & $4 A 7$ & 2.64 & 1225 \\
\hline Juno & 0.29 & 5.7 & 022 & 0.00 & 432 & 2.55 & 1264 \\
\hline Juty & 0.29 & 5.99 & 022 & 0.00 & 447 & 2.64 & 13.09 \\
\hline August & 029 & 5.34 & 022 & 0.00 & 447 & 2.64 & 1244 \\
\hline Septomber & 0.29 & 498 & 022 & 0.00 & 432 & 297 & 1228 \\
\hline October & 0.29 & 3.95 & 022 & 0.00 & $4 A 7$ & 3.60 & 1202 \\
\hline November & 0.29 & 2.82 & 022 & 0.56 & 432 & 4.08 & 11.79 \\
\hline Decomber & 0.00 & 0.00 & 022 & 1.82 & 4.47 & 5.36 & 11.65 \\
\hline TOTALm2 & & 38.19 & & 6.84 & 5260 & 4293 & 140.56 \\
\hline
\end{tabular}


OPTION 2:

SEMLTRANSPARENT DOUBLE GLAZED FV ROOF

STE DATA

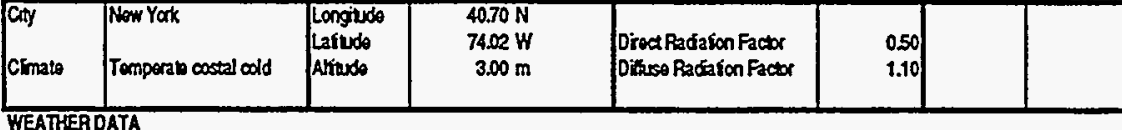

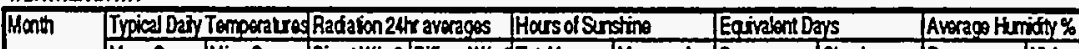

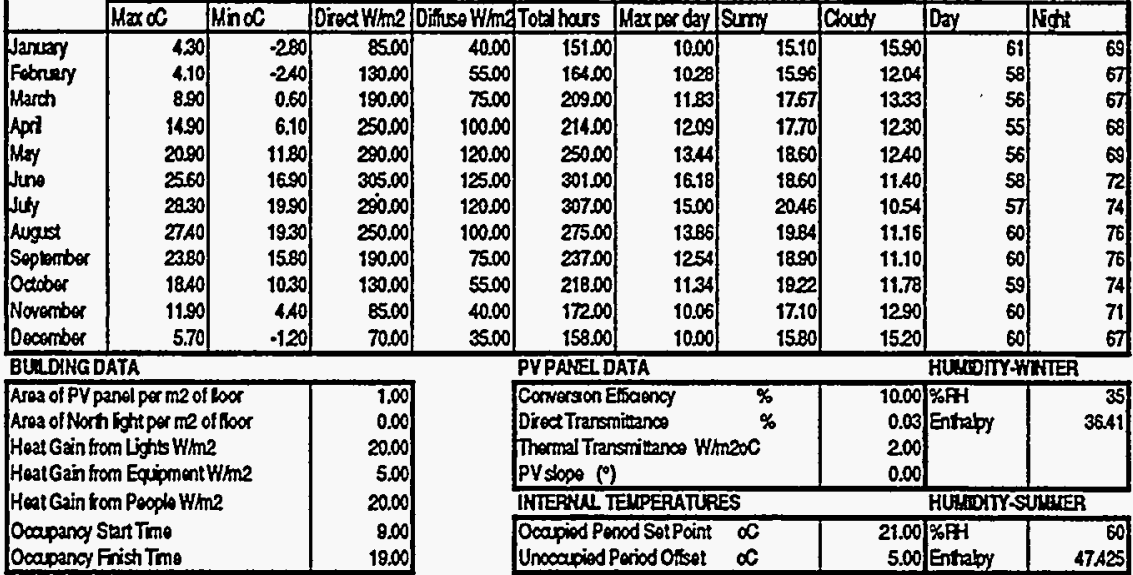

CALCULATIOHS OF MESH EXTEPUL CONDITIONS

\begin{tabular}{|c|c|c|c|c|c|c|c|c|c|c|}
\hline \multirow[t]{2}{*}{ Month } & \multicolumn{2}{|c|}{ Temperature oc } & \multicolumn{6}{|c|}{ 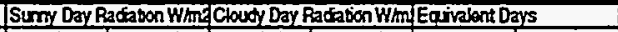 } & \multicolumn{2}{|c|}{ IExtExtaloy $k$ klog } \\
\hline & Ocoupied & thocoloiad & oocupiod & Uhocaniod & loopiod & Lhocapiod & Simy & Cousty & Docupiod & Throcanoiod \\
\hline January & 253 & -0.52 & 207.60 & 0.00 & 96.00 & 0.00 & 15.10 & 1590 & 895 & 480 \\
\hline =ebruary & 248 & $-0,31$ & 293.12 & 5.77 & 128,46 & 253 & 1596 & 1204 & 856 & 4.99 \\
\hline 由) & 6.83 & 327 & 360.16 & 47.03 & 152.18 & 1987 & 17.67 & 1333 & 15.67 & 10.86 \\
\hline & 1270 & 8.93 & 456,49 & 69.65 & 198.50 & 29.64 & 17.70 & 1230 & 26.71 & 2580 \\
\hline$y$ & 18.53 & 14.73 & 484.61 & 121.56 & 21427 & 5266 & 1860 & 1240 & 39.98 & 3506 \\
\hline $\sin \theta$ & 2343 & 19.70 & 430.09 & 169.94 & -185.38 & 81.87 & 1850 & $11 A 0$ & 5274 & 49.03 \\
\hline Juty & 2620 & 2260 & 443.06 & 158.39 & 191.34 & 6862 & 2046 & 1054 & 59.60 & 5833 \\
\hline Augest & 2538 & 21800 & 40680 & 11221 & 173.15 & 47.75 & 1984 & 11.16 & 5906 & 57.18 \\
\hline Septomber & 2180 & 1837 & 330.72 & 61.63 & 14354 & 25.04 & 1890 & 11.10 & 49,48 & 4690 \\
\hline Databer & 1638 & 1290 & 265.55 & $\mathbf{2 5 . 4 6}$ & 116.38 & 11.16 & 1822 & 11.78 & 3584 & 3204 \\
\hline & 10.03 & 6.81 & 20630 & 086 & $\$ 5.44$ & 0.40 & 17.10 & 1290 & 2245 & 18.01 \\
\hline Docombor & 3.98 & 1,02 & 17640 & 0.00 & 84.00 & .0 .00 & 1580 & 1520 & 1126 & 700 \\
\hline
\end{tabular}

SUATY DAYHEAT BALAKCE

\begin{tabular}{|c|c|c|c|c|c|c|c|c|c|c|c|}
\hline \multirow[t]{2}{*}{ Manth } & \multicolumn{3}{|c|}{ loogond Period W/m2 } & \multicolumn{3}{|c|}{ Unocangiod Period Hhin2 } & \multicolumn{5}{|c|}{ Monthy Requirement kWh Fresh Á Lasd kWh } \\
\hline & PVnotgin & Intarals & Ovaral & PV notgain & Intema's & OMarl & Heating & Cooing & Heaing & Cooing & \\
\hline Jantay & $-18 A 5$ & 2500 & 555 & 3304 & 450 & -2854 & -603 & 084 & -7.96 & & 0.00 \\
\hline Fobrey & -1234 & 2500 & 1266 & 2213 & 450 & -27.63 & 6.17) & 2.02 & -254 & & 0.00 \\
\hline an & 201 & 2500 & 27.01 & -21.50 & 450 & -17.00 & 421 & $4 . \pi$ & -7.04 & & 0.00 \\
\hline pri & 2272 & 25.00 & 47.72 & -827 & 450 & -3.77 & -0.83 & 8.45 & 330 & & 0.00 \\
\hline $\mathrm{Mzy}$ & 36.95 & 25.00 & 61.95 & -230 & 450 & 220 & 0.00 & 1209 & 0.00 & & 0.00 \\
\hline Jine & 41.11 & 25.00 & 66.11 & 6.74 & 450 & 1124 & 0.00 & 1522 & 0.00 & & 1.90 \\
\hline & 47.75 & 2500 & 7275 & 6.55 & 450 & 11.05 & 0.00 & 1805 & 0.00 & & 4.78 \\
\hline August & 4305 & 2500 & 6805 & 460 & 450 & 8.10 & 0.00 & 16.03 & 0.00 & & 4,43 \\
\hline ber & 3024 & 2500 & 5524 & -0.06 & 450 & $4 / 4$ & .0 .00 & 11.61 & 0.00 & & 0.75 \\
\hline & 13.14 & 25.00 & 38.14 & 405 & 450 & 0.45 & 0.00 & 745 & -021 & & 0.00 \\
\hline Nowamber & 455 & 2500 & 20.45 & $-18,31$ & 450 & -9381 & 331 & 3.50 & -458 & & 0.00 \\
\hline Decomber & -19.18 & 25.00 & 582 & -29.95 & 450] & -25.46 & 5.63 & 0.82 & -7.6 & & 0.00 \\
\hline \multicolumn{12}{|c|}{ CLOLOY DAY KEAT BALAHCE } \\
\hline \multirow[t]{2}{*}{ Manth } & \multicolumn{3}{|c|}{ Ooapiod PeriodW/m2 } & \multicolumn{3}{|c|}{ Unocapiod Poriod Hinn? } & \multicolumn{5}{|c|}{ Monthy Bogr remontkWh Fresti Ar LosdkWh } \\
\hline & PVnetgain & Inteme's & Owall & PVnatgain & Intemals & Overel & Heaing & Cooing & Heaing & $\operatorname{cosing}$ & \\
\hline Januery & .28 .86 & 45.00 & 16.14 & -33.04 & 450 & -28.54 & 635 & 257 & -8338 & & 0.00 \\
\hline Fobruary & -2622 & 40.00 & 1378 & $-321 t$ & 450 & -27.91 & -4.70 & 1.66 & 54 & & 0.00 \\
\hline Warch & -15.52 & 35.00 & $18 A B$ & -23.78 & 450 & -1929 & 2600 & 2.60 & 531 & & 0.00 \\
\hline Apt? & 0.13 & 30.00 & 30.13 & -11.64 & 450 & -7.14 & -123 & 3.71 & -229 & & 0.00 \\
\hline Hay & 1331 & 2500 & 3831 & 1180 & 450 & 6.30 & 0.00 & 586 & 0.00 & & 0.00 \\
\hline June & 20,18 & 25.00 & 45,48 & 329 & 450) & 7.78 & 0.00 & 6.43 & 0.00 & & 1.16 \\
\hline Juty & 2658 & 25.00 & 51.58 & 3.98 & 4.50 & 8.48 & 0.00 & 6.69 & 0.00 & & 246 \\
\hline Auge: & 2335 & 25.00 & 48.35 & 250 & 450 & 7.00 & 0.00 & $6 A 9$ & 0.00 & & 249 \\
\hline Soptonber & 13.70 & 30.00 & 43.70 & -206 & 450 & 244 & 0.00 & 523 & 0.00 & & 0.44 \\
\hline & 0.56 & 35.00 & 3556 & 525 & 450 & -0.75 & -0.12 & 4.19 & -0.83 & & 0.00 \\
\hline Now & -13.90 & 40.00 & 26.10 & -18.34 & 450 & -1384 & -250 & 3.37 & -346 & & 0.00 \\
\hline Decomber & -26.97 & 45.00 & 18.03 & -29.96 & 450 & -25.46 & -5.42 & 2.74 & -7.34 & & 0.00 \\
\hline
\end{tabular}

OVERALI ERERGYREQUREUEKTS APD PRODUCTON

\begin{tabular}{|c|c|c|c|c|c|c|c|}
\hline \multirow[t]{2}{*}{ Manth } & \multicolumn{2}{|c|}{ Coding Consumpion } & \multicolumn{2}{|c|}{ Healing Consurmpion } & \multirow{2}{*}{$\begin{array}{l}\text { Fans } \\
\text { kWh }\end{array}$} & \multirow{2}{*}{$\begin{array}{l}\text { Lighing and } \\
\text { Power kWh }\end{array}$} & \multirow{2}{*}{$\begin{array}{l}\text { Tobalkh } \\
\text { Consemod }\end{array}$} \\
\hline & 1600 & Km Boctic & $1 / 000$ & kth Blatic & & & \\
\hline Jaxuay & 0.00 & 0.00 & 022 & 6.38 & 5.04 & 5.82 & 172 \\
\hline Fobruary & 0.00 & 0.00 & 022 & 5.75 & 455) & 4.19 & 144 \\
\hline March & 0.00 & 0.00 & 022 & $4 A 8$ & 5.04 & 3.97 & 1348 \\
\hline Apri & 0.00 & 0.00 & 022 & 1.72 & 487) & 3.17 & $9 . \pi$ \\
\hline May & 029 & 5.13 & 022 & 0.00 & 5.04 & 264 & 12 \\
\hline June & 028 & 7.06 & 022 & 0.00 & 4.87 & 255 & $14 \mathrm{~A}$ \\
\hline Judy & 029 & 9.14 & 022 & 0.00 & 5.04 & 2.64 & 1681 \\
\hline August & 029 & 841 & 022 & 0.00 & 5.04 & 2.64 & 16.0 \\
\hline Septonber & 029 & 5.15 & 022 & 0.00 & 4.87 & 3.11 & 13 \\
\hline Oatcour & 029 & 3.33 & 022 & 0.10 & 5.04 & 381 & 1228 \\
\hline Novernber & 0.00 & 0.00 & 0.22 & 3.08 & 4.87 & 449 & 1244 \\
\hline Oocomber & 0.00 & 0.00 & 022 & 5.78 & 5.04 & 5.68 & 1649 \\
\hline TOTALm2 & & 3820 & & 2730 & 5928 & 44,67 & $169 \mathrm{~A}$ \\
\hline
\end{tabular}


OPTION 2: SEMLTRANSPARENT DOUBLE GLAZED PV ROOF

STEDATA

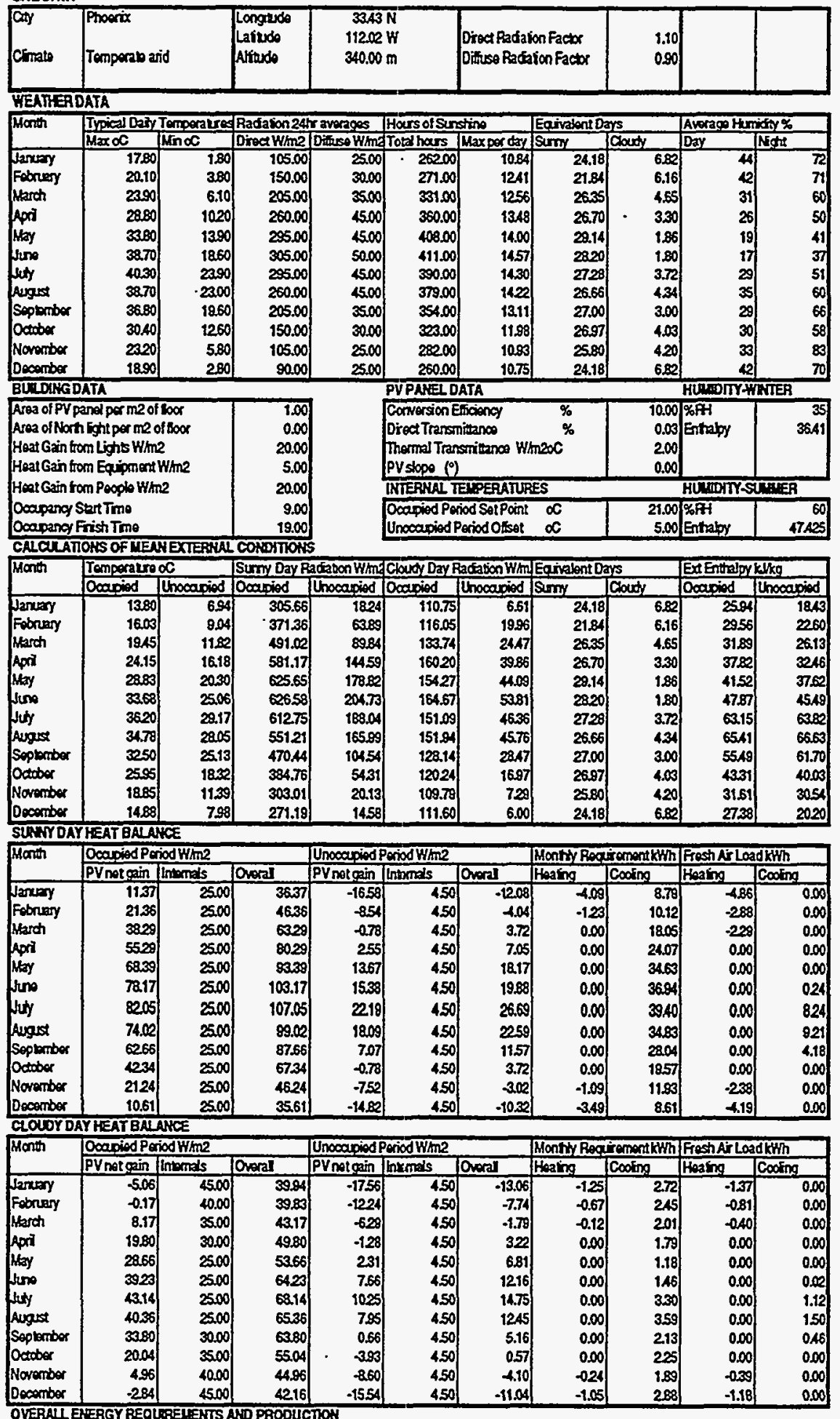

OVERAII ERERGY REQLRE IEETS AKD PRODUCTION

\begin{tabular}{|c|c|c|c|c|c|c|c|}
\hline \multirow[t]{2}{*}{ Henth } & \multicolumn{2}{|c|}{ Coding Consumpion } & \multicolumn{2}{|c|}{ Heating Consumpoin } & \multirow{2}{*}{$\begin{array}{l}\text { Frns } \\
\text { kWh }\end{array}$} & \multirow{2}{*}{$\begin{array}{l}\text { Lighting and } \\
\text { Power kWh }\end{array}$} & \multirow{2}{*}{$\begin{array}{l}\text { TobikWh } \\
\text { Consemod }\end{array}$} \\
\hline & $1 / \cos$ & Kthn Eectic & $1 / 000$ & kth Eloctic & & & \\
\hline Jantery & 0.00 & 0.00 & 022 & 2.57 & 786 & 400 & 14 \\
\hline fobruesy & 0.20 & 359 & 022 & 124 & 7.10 & 3.30 & 15 \\
\hline Harch & 0.29 & 5.73 & 0.22 & 0.62 & 786 & 3.10 & או \\
\hline Aprit & 0.28 & 739 & 022 & 0.00 & 7.60 & 272 & \\
\hline May & 029 & 1023 & 022 & 0.00 & 786 & 2.64 & \\
\hline fine & 029 & 11.05 & 0.22 & 0.00 & 7.60 & 255 & \\
\hline Wuy & 0.29 & 1487 & 022 & 0.00 & 7.86 & 264 & 25 \\
\hline August & 029 & 14.04 & 022 & 0.00 & 786 & 2.64 & 24 \\
\hline Septerniber & 0.28 & 8.95 & 022 & 0.00 & 7.60 & 270 & 20 \\
\hline Oetcoer & 029 & 623 & 0.22 & 0.00 & 7.86 & 3.04 & 17. \\
\hline Noromber & 0.29 & 3.5 & 0.22 & 0.91 & 7.60 & 3.18 & 15.6 \\
\hline Decomber & 0.00 & 0.00 & 022 & 221 & 786 & 4.00 & 14.06 \\
\hline TOTAL/m2 & & 87.03 & & 755 & 9252 & 36.49 & 2232 \\
\hline
\end{tabular}


SMEDATA

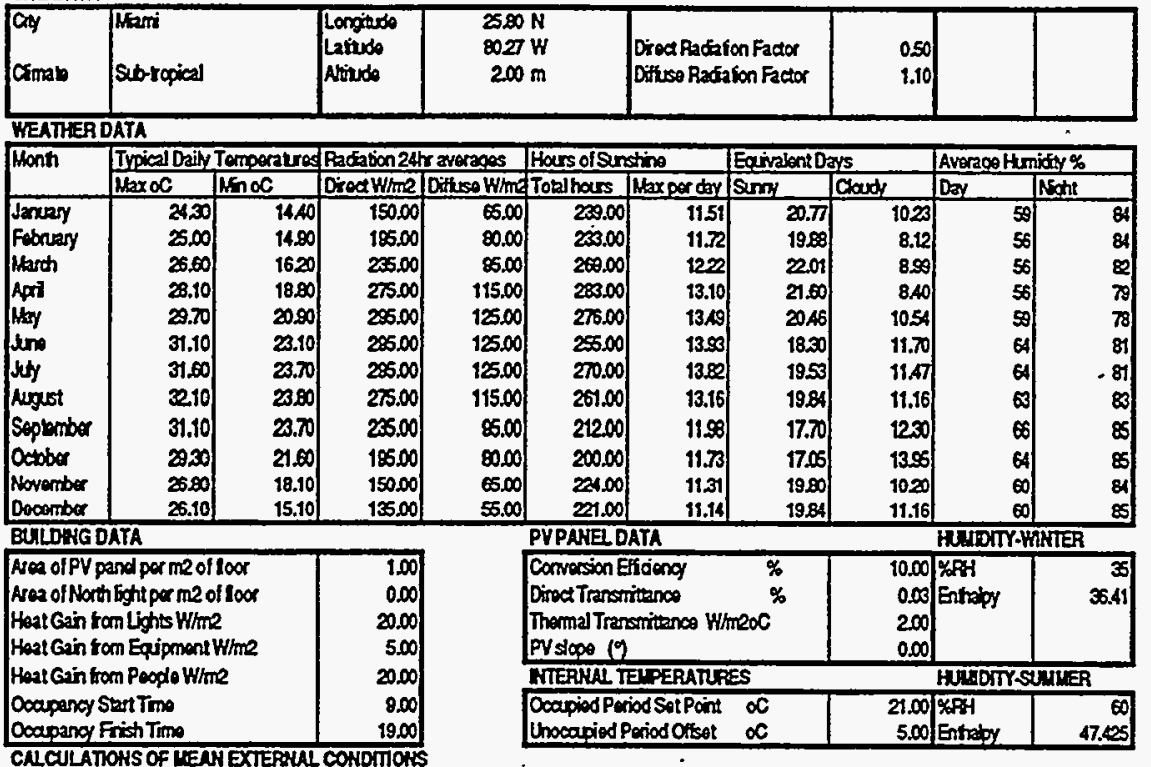

CALCULATIOHS OF LENH EXTEPYAL COHDTIOHS

\begin{tabular}{|c|c|c|c|c|c|c|c|c|c|c|}
\hline \multirow[t]{2}{*}{ Month } & \multicolumn{2}{|c|}{ Temperairo $\alpha$} & \multicolumn{6}{|c|}{ 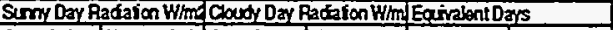 } & \multicolumn{2}{|c|}{ EntEnhaby klkg } \\
\hline & Osapied & Unocospiod & Cocapiad & Unocapiod & Ocapiod & Uhocapind & Suny & Cand & Osapiod & thooapiod \\
\hline January & 21.83 & 1758 & 30555 & 3028 & 135.57 & H.50 & $20 . \pi$ & 1023 & 49.08 & 475 \\
\hline & 2208 & 18.15 & 37.85 & 46.68 & 16328 & 20.13 & 19.88 & 8.12 & 4932 & 4023 \\
\hline तक & 26,00 & 1954 & 45.25 & 60.18 & 18655 & 20.60 & 2201 & 890 & 5321 & 5254 \\
\hline$n$ & 25.78 & 21.78 & 483.60 & 107.15 & 210.66 & 45.57 & 21.50 & 8.40 & 588 & 58.21 \\
\hline Noy & 2750 & 23.73 & 507.05 & 126.39 & 22239 & 5013 & 20.46 & 10.54 & 6443 & 6380 \\
\hline un & 29.10 & 25.67 & 490.87 & 137.25 & 21529 & 6050 & 18.30 & 11.50 & 7210 & 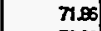 \\
\hline Juty & 20.03 & 2624 & 494.76 & 135.17 & 217.00 & 5020 & 19.53 & 11,47 & 74,06 & 73.80 \\
\hline Augut & 30.03 & 26,97 & 461.53 & 108.55 & 20080 & 4728 & 1984| & 11.15 & 74.60 & 7.78 \\
\hline Sepenber & 2025 & 25.08 & 4484 & 2283 & 19038 & 2689 & 17.00 & 1230 & 7426 & 7.58 \\
\hline actober & 2738 & 24.08 & 372.53 & 46.91 & 163.58 & 2020 & 17.05 & 13.95 & 67.19 & 68.00 \\
\hline Novernber & 24.53 & 20.90 & 310.70 & 29.15 & 137.89 & 1298 & 19.80 & 1020 & 57.00 & 57.57 \\
\hline Docomber & 23.25 & 18.64 & 25.78 & $2 \mu$ & 118.50 & 8.64 & 19.84 & 11.16 & 59.56 & 51.08 \\
\hline
\end{tabular}

SRIWY DAY FEAT BALAHCE

\begin{tabular}{|c|c|c|c|c|c|c|c|c|c|c|}
\hline \multirow[t]{2}{*}{ Month } & \multicolumn{3}{|c|}{ Ooonoid Poriod W/me } & \multicolumn{3}{|c|}{ Uhocapiod Poriod Wim? } & \multicolumn{2}{|c|}{ Montly Rogirement kWh } & \multicolumn{2}{|c|}{ Frosh Air Load kHh } \\
\hline & PVnolqin & herrots & Overall & PYmolgan & hivmals & Operil & Hoaing & Cooing. & Heating & $\cos \cos ^{2}$ \\
\hline Jancery & 27A1 & $25 \infty 0$ & 5241 & 206 & 450 & 24 & 0.00 & 11.50 & $\overline{0.00}$ & 0.06 \\
\hline any & 197 & 2500 & 50.87 & 023 & 450 & 473 & 0.00 & 1324 & 0.00 & 0.72 \\
\hline Werch & $2 \pi$ & 2500 & 67.5 & 192 & 450 & 6,2 & 0.00 & 1620 & 0.00 & 245 \\
\hline Apri & 5032 & 25.00 & 7532 & 5.57 & 450 & 10.11 & 0.00 & 1933 & 0.00 & 434 \\
\hline Why & 5.74 & 2500 & 80.74 & 9.45 & 450 & 13.95 & 0.00 & 20.51 & 0.00 & $6 \infty 8$ \\
\hline ino & 5758 & 2500 & 8258 & 1097 & 450 & $15 \pi 7$ & 0.00 & 18.08 & 0.00 & 8.78 \\
\hline Jubs & 58.26 & 2500 & 83.96 & 1187 & 450 & 16.37 & 0.00 & 2087 & 0.00 & 999 \\
\hline Aungs & 58.65 & 25.00 & 83.05 & 10.00 & 450 & 1458 & 0.00 & 20.5 & 0.00 & 10.35 \\
\hline Seplonber & 54.00 & 2500 & 7900 & 5.45 & 450 & 9.95 & 0.00 & 1645 & 0.00 & 9.12 \\
\hline actobe & 4.74 & 2500 & $\boldsymbol{\oplus . 7 4}$ & $3 \mu$ & 450 & $78 x$ & 0.00 & 13.79 & 0.00 & 6.97 \\
\hline Nover & $33 / 5$ & 2500 & FAS & 0.25 & 450 & 4.75 & 0.00 & 1289 & 0.00 & 3.64 \\
\hline Decomber & 27.56 & 25.00 & 5295 & -0.84 & 4.50 & 3.66 & 0.00 & 11.52 & 0.00 & 234 \\
\hline
\end{tabular}

CLOUDYDAYHEATBNLAKCE

\begin{tabular}{|c|c|c|c|c|c|c|c|c|c|c|}
\hline \multirow[t]{2}{*}{ Monh } & \multicolumn{3}{|c|}{ Ocoupiod Period W/m2 } & \multicolumn{3}{|c|}{ Itrocapiod Penod W/m2 } & \multicolumn{2}{|c|}{ Mon'hy Regirernent kth } & \multicolumn{2}{|c|}{ Frash Air Load kHh } \\
\hline & PV notgain & himernats & Overal & PV notgain & hrtals & Overel & Heaing & $\cos ^{2} n$ & Hoaing & $\cos x$ \\
\hline Janey & 13.08 & 4500 & 58.08 & -0.61 & 450 & 36 & 0.00 & 650 & 0.00 & 0,33 \\
\hline Februay & 16.7 & 40.00 & 56.76 & -0.68 & 450 & $3: 2$ & 0.00 & 5.04 & 0.00 & 0.30 \\
\hline March & 21.70 & $\mathbf{3 5 . 0 0}$ & 56.70 & 0.58 & 450 & 500 & 0.00 & 5.74 & 0.00 & 100 \\
\hline Apri & 2731 & $\mathbf{3 0 \infty}$ & 5731 & 218 & 4.50 & 6.50 & 0.00 & 500 & 0.00 & 1.00 \\
\hline Moy & 3.5 & 25,00 & 56.75 & 346 & 450 & 720 & 0.00 & 7.16 & 0.00 & $3 / 4$ \\
\hline $\sin$ & 345 & 25.00 & 5935 & 4M & 450 & 89: & 0.00 & 8A1 & 0.00 & 5.02 \\
\hline$J \omega_{y}$ & 35.54 & $25, \infty$ & cost & $5 A B$ & 450 & 9.98 & 0.00 & 8.55 & 0.00 & 506 \\
\hline Algost & 35.74 & 25.00 & 60.74 & 4.28 & 450 & 812 & 0.00 & 825 & 0.00 & 5.28 \\
\hline Sepronber & 3255 & $\mathbf{3 0 . 0 0}$ & 255 & $2 / 2$ & 450 & 692 & 0.00 & 8E9 & 0.00 & 634 \\
\hline Octobar & 2655! & 3500 & 6155 & 1.19 & 450 & $5 . \infty$ & 0.00 & 9.70 & 0.00 & 520 \\
\hline Noveriber & 1887 & 40.00 & 58.87 & -0.12 & 450 & 438 & 0.00 & 6.53 & 0.00 & 157 \\
\hline Decomber & 14.00 & 15.00 & 50.60 & -0.58 & 450 & 3.5 & 0.00 & 727 & 0.00 & 131 \\
\hline
\end{tabular}

OVERALL ENERGY PECURE VENTS AND PRODUCTION

\begin{tabular}{|c|c|c|c|c|c|c|c|}
\hline \multirow[t]{2}{*}{ Month } & \multicolumn{2}{|c|}{ Coosing Consumpion } & \multicolumn{2}{|c|}{ Thearing Consampion } & \multirow{2}{*}{$\begin{array}{l}\text { Fans } \\
\text { ixhn }\end{array}$} & \multirow{2}{*}{$\begin{array}{l}\text { Lighing and } \\
\text { Poworkthe }\end{array}$} & \multirow{2}{*}{$\begin{array}{l}\text { Toalkwh } \\
\text { Consemod }\end{array}$} \\
\hline & $1 / \log 0$ & KWh Bectic & $1 \log 0$ & Kkm Eoctic & & & \\
\hline Janery & 020 & $5 / 5$ & 022 & 0.0 & 629 & 4.68 & 16,42 \\
\hline Fobruary & $028]$ & 5.51 & 022 & 0.00 & 5.68 & $3 \infty 0$ & 14.79 \\
\hline March & 020 & 745 & 022 & 0.00 & 620 & 350 & 1727 \\
\hline Apri & 022 & 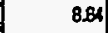 & 022 & 0.00 & 600 & 297 & 17.90 \\
\hline Mry & 028 & 9020 & 022 & 0.00 & 620 & 264 & 19.72 \\
\hline tho & 020 & 11.97 & 022 & 0.00 & $6 \infty$ & 255 & 20.00 \\
\hline Juty & 028 & 12.28 & 022 & 0.00 & 620 & 264 & 218 \\
\hline Alugest & 029 & 1288 & 022 & 0.00 & 62 & 264 & $2 t .00$ \\
\hline Scotenter & 028 & 11.05 & 022 & 0.00 & $6 \infty$ & 3.17 & 20.9 \\
\hline actober & 0.28 & 10.07 & 022 & 0.00 & 629 & 4.08 & 2020 \\
\hline November & 028 & 7.15 & 022 & 0.00 & 6.00 & 400 & 1732 \\
\hline Decumber & 0.28 & 6A1 & 0.22 & 0.00 & 620 & 487 & 1757 \\
\hline TOTAL/m2 & & 111.12 & & 0.00 & 74.06 & 1133 & 225.56 \\
\hline
\end{tabular}


OPTION 2: SEMITRANSPARENT DOUBLE GLAZEO PV ROOF

\begin{tabular}{|c|c|c|c|c|c|c|c|c|c|c|}
\hline Citis & \multicolumn{2}{|c|}{$\begin{array}{l}\text { Cricago } \\
\text { Tomperaw coninental }\end{array}$} & $\begin{array}{l}\text { Longitude } \\
\text { Lafude } \\
\text { Afsurdo }\end{array}$ & $\begin{array}{r}41.78 \\
87.75 \\
186.00\end{array}$ & & \multicolumn{2}{|c|}{$\begin{array}{l}\text { Direct Radaron Factor } \\
\text { Dituso Padation Factor }\end{array}$} & $\begin{array}{l}0.50 \\
1.10\end{array}$ & & \\
\hline \multicolumn{11}{|c|}{ WEATHERDATA } \\
\hline \multirow[t]{2}{*}{ Month } & \multicolumn{2}{|c|}{ Trpical Dajy Termperalres } & \multicolumn{2}{|c|}{ Radaton $24 \mathrm{hr}$ averages } & \multicolumn{2}{|c|}{ Thors of sunstine } & \multicolumn{2}{|c|}{ Equialont Dass } & \multicolumn{2}{|c|}{ Averecs Humidit; } \\
\hline & Max $\propto C$ & $\operatorname{Min} \alpha$ & Direct W/m2 & Difissowhe & Totihours & Haxpordy & Sumy & Couty & Day & Night \\
\hline Januey & 0.60 & -720 & 85.00 & 40.00 & 12500 & 10.00 & 1250 & 1850 & 70 & 80 \\
\hline fooruary & 150 & -630 & 130.00 & 5500 & 141.00 & $10 \times 4$ & $13 / 4$ & 1456 & 67 & 79 \\
\hline Manch & 640 & +1.70 & 19000 & 7500 & 206.00 & 1278 & 16.12 & 1488 & 61 & $\pi$ \\
\hline Hat & 14.10 & 4.70 & 250.00 & 10000 & 206.00 & 1248 & 16.50 & 1350 & 56 & 74 \\
\hline 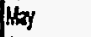 & 20.60 & 1050 & 290.00 & 20.00 & 275.00 & 1454 & 1891 & 1209 & 5 & 75 \\
\hline $\operatorname{lin} \theta$ & 2640 & 15.40 & 30500 & 125.00 & 30700 & 1551 & 1980 & 1020 & 56 & $n$ \\
\hline Wy & 2890 & 1950 & 29000 & 120.00 & 31000 & 1389 & 2232 & 8.08 & 53 & $\pi$ \\
\hline August & 2300 & 1880 & 250.00 & 100.00 & 285.00 & 13.13 & 21.70 & 830 & 56 & 81 \\
\hline Seppuntor & 2380 & 14.10 & 180.00 & 7500 & 246.00 & 1212 & 1980 & 1020 & 53 & 89 \\
\hline Oatober & 1740 & 820 & 130.00 & 5500 & 214.00 & 11.13 & 1920 & 11.78 & SA & 7 \\
\hline Norember & 840 & 0.30 & 8500 & 4000 & 135.00 & 10.00 & 1350 & 1650 & 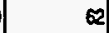 & 78 \\
\hline Docomber & 210 & 530 & 70.00 & 3500 & 115.00 & 10.00 & 11.50 & 1950 & 69 & 80 \\
\hline \multicolumn{4}{|c|}{ BLCOMGDATA } & & PYPAKE D & SATA & & & HIT'DITH & VNIER \\
\hline $\begin{array}{l}\text { Arez of PV } \\
\text { Aroa of Nar } \\
\text { Heat Gaint } \\
\text { Heat Gaint }\end{array}$ & 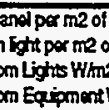 & 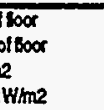 & $\begin{array}{r}1.00 \\
0.00 \\
20.00 \\
5.00\end{array}$ & & $\begin{array}{l}\text { Cormorsion E } \\
\text { Direct Transm } \\
\text { Thermal Tran } \\
\text { PV siope (o) }\end{array}$ & $\begin{array}{l}\text { Eliciency } \\
\text { mitarios } \\
\text { nsmitano Wh } \\
\text { ) }\end{array}$ & ${ }^{\%}$ & $\begin{array}{l}10.00 \\
0.03 \\
2.00 \\
0.00\end{array}$ & Entapy & $\begin{array}{r}35 \\
36.41\end{array}$ \\
\hline Hongant & om Pooglo W/h & & 2000 & & INTERNIL T & IETPEATIR & & & HUT:DTS & DIII \\
\hline $\begin{array}{l}\text { Oospanof } \\
\text { Oocopany }\end{array}$ & $\begin{array}{l}\text { SertTmo } \\
\text { irish Tme }\end{array}$ & & $\begin{array}{r}9000 \\
19,00\end{array}$ & & $\begin{array}{l}\text { Ocapiod Peri } \\
\text { Unocopiod P }\end{array}$ & $\begin{array}{l}\text { riod SalPoint } \\
\text { Period Otsot }\end{array}$ & $\underset{\alpha}{\alpha}$ & $\begin{array}{r}21.00 \\
5.00\end{array}$ & $\begin{array}{l}\text { \%FF } \\
\text { Entraby }\end{array}$ & $\begin{array}{r}60 \\
47125\end{array}$ \\
\hline chetert & OWS OFMED & NEXTERAU & CONDTrots & & - & & & & & \\
\hline Manth & Temperatro & & Sumy Day & GébonWhr? & Cloudy Day & Ragabon Wha & Egrvalent Das & & ExtEntralpy & Whag \\
\hline & oocuniod & Utroosuiod & Oocapiod & Thoosipiad & Docupind & Uhooariod & Surmy & Coudy & Docuiod & thooconiad \\
\hline Jantry & -135 & 4.69 & 207.60 & 0.00 & 86.00 & 0.00 & 1250 & 1850 & $3 . \infty$ & -0.55 \\
\hline Fobruery & $-0,45$ & 379 & 287.10 & 10.07 & 125.82 & 4.41 & 13,4 & 1456 & 4.78 & 0.64 \\
\hline March & 438 & 0.90 & 353.36 & 66.17 & 140.85 & 2796 & 16.12 & 1488 & 1207 & 780 \\
\hline Apri & 11.75 & 7.72 & 451.75 & 80.18 & 18223 & 34.12 & 16.50 & 1350 & 2503 & 2036 \\
\hline Noy & 1808 & 13.75 & 457.14 & 14833 & 188.04 & 6426 & 1891 & 1200 & 3834 & 3437 \\
\hline fino & 23.65 & 1894 & 448.89 & 176.51 & 18348 & 76.08 & 19.80 & 1020 & 5231 & 4988 \\
\hline frity & 2655 & 2252 & 478.56 & 13298 & 20736 & 57.60 & 2232 & 8.68 & 58.17 & 5953 \\
\hline fougst & 25.70 & 21.76 & $429 A 3$ & 86.12 & 182.74 & 40.50 & 21.70 & 9.30 & 57.68 & 59.08 \\
\hline Scpoteriber & 2138 & 1722 & 34288 & 59.37 & 14488 & 25.09 & 1980 & 1020 & 4523 & 15.46 \\
\hline Octuber & 15.10 & 11.16 & 270.52 & 21.92 & 118.55 & 0.60 & 1922 & 11.78 & 31,44 & 29.05 \\
\hline Norember & 6.38 & 290 & 207.60 & 0.00 & 9600 & 0.00 & 1350 & 16.50 & 1578 & $11 / 13$ \\
\hline Docontiber & 0.25 & -292 & $176 \wedge 10$ & 0.00 & 84.00 & 0.00 & 1150 & 1950 & 6.03 & 1.95 \\
\hline SLFAY & HERT BALA & ACE & & & & & & & & \\
\hline Manth & Ooapiod $P$ o & $\operatorname{cod} W / \mathrm{m}^{2}$ & & Unocapiod P & PeriodW/m2 & & Monthy Regi & IranentkWh & IFrosh Ár Loa & $d \mathrm{dWh}^{2}$ \\
\hline & PVnotgin & tontrinats & Ouxcal & pVnotgain & Inkmals & Overal & Hoaing & Cooling & Hoaing & Cooting \\
\hline Jaruay & -2720 & 25.00 & -220 & 4139 & 450 & 3689 & -646 & -027 & -788 & 0.00 \\
\hline Fobruery & -18.70 & 25.00 & 630 & -38.74 & 450 & -3424 & $-6,4$ & 0.85 & 216 & 0.00 \\
\hline Narch & 5.15 & 25.00 & 1805 & -24.61 & 450 & -20.11 & 451 & 320 & -754 & 0.00 \\
\hline Apri & 1958 & 2500 & 4558 & -280 & 450 & -5.30 & -122 & 736 & -361 & 0.00 \\
\hline Ity & 3269 & 25.00 & 57.69 & 300 & 450 & 750 & 0.00 & 1200 & 0.00 & 0.00 \\
\hline Jin & 43.14 & 2500 & 68.14 & 712 & 450 & 1152 & 0.00 & 1680 & 0.00 & 1.86 \\
\hline Juty & 51.45 & 2500 & 7615 & 7.58 & 450 & 1208 & 0.00 & 2084 & 0.00 & 4.61 \\
\hline August & 4560 & 2500 & 70.60 & 4.62 & 450 & 0.12 & 0.00 & 1800 & 0.00 & 427 \\
\hline Soptariber & 29.65 & 25.00 & 54.65 & 244 & 450 & 6.84 & 0.00 & 1275 & 0.00 & 0.00 \\
\hline Octabor & 11.00 & 2500 & 3500 & -784 & 4.50 & -334 & -0.90 & 6.52 & -184 & 0.00 \\
\hline Nowember & -11.75 & 25.00 & 1325 & -25.19 & 450 & -21.69 & 4.10 & 1.78 & -535 & 0.00 \\
\hline Decomber & -26.63 & 25.00 & -1.63 & -3784 & 4.50 & .33 .34 & -537 & -0.19 & -6.71 & 0.00 \\
\hline CLOWY & YYEATBAL & ANCE & & & & & & & & \\
\hline Manth & Ocapiad Pen & iodW/m2 & & UnocapiadP & eriodW/n2 & & Monthy Rege & foment $k$ hn & FroshAir Lo3 & th \\
\hline & PVnatgin & Internals & Ororal & PVratgain & Inbrels & Overal & Hosing & Cooling & Hoaty & Cooing \\
\hline Januery & 36.51 & 45.00 & 839 & 4139 & 450 & 3689 & -955 & 1.55 & -11.64 & 0.00 \\
\hline Fobrupy & 5229 & 40.00 & 7.71 & 2021 & 450 & 34.71 & -7.00 & 1.12 & -884 & 0.00 \\
\hline March & -21.38 & 3500 & 13.62 & -2784 & 450 & -2334 & -486 & 203 & -696 & 0.00 \\
\hline Apri & -220 & 30.00 & 27.71 & -1368 & 450 & -9.18 & -1.74 & 3.74 & -2.95 & 0.00 \\
\hline May & 10.84 & 2500 & 3584 & 0.91 & 4.50 & 5A1 & 0.00 & 525 & 0.00 & 0.00 \\
\hline Jino & 21.51 & 2500 & 46.61 & 320 & 450 & 7.79 & 0.00 & 587 & 0.00 & 0.96 \\
\hline hity & 2858 & 2500 & 5358 & 290 & 4.50 & 7.40 & 0.00 & 555 & 0.00 & 1.79 \\
\hline August & 24.80 & 2500 & 4980 & 1.63 & 4.50 & 6.13 & 0.00 & $5 / 3$ & 0.00 & 1.83 \\
\hline Sepromber & 1208 & 30.00 & 4296 & -0.45 & 450 & 4.05 & 0.00 & 496 & 0.00 & 0.00 \\
\hline Octobor & -1.81 & 3500 & 33.10 & -288 & 450 & -438 & -0.72 & 3.91 & -1.13 & 0.00 \\
\hline Novermbor & -21.16 & 40.00 & 1884 & -26.10 & 4.50 & -21.69 & -5.01 & 3.11 & 6.54 & 0.00 \\
\hline Docamber & 34.42 & 45.00 & 10.58 & .37 .84 & 450 & -39.34 & -9.10 & 206 & $-11,38$ & 0.00 \\
\hline
\end{tabular}

OVERALL ENERGYREQLREVENTS AND PRODUCTIOH

\begin{tabular}{|c|c|c|c|c|c|c|c|}
\hline \multirow[t]{2}{*}{ Month } & \multicolumn{2}{|c|}{ Coding Consumption } & \multicolumn{2}{|c|}{ Heating Consumpion } & \multirow{2}{*}{$\begin{array}{l}\text { Fans } \\
\text { kym }\end{array}$} & \multirow{2}{*}{$\begin{array}{l}\text { Lighing and } \\
\text { Power KW/h }\end{array}$} & \multirow{2}{*}{$\begin{array}{l}\text { Total kWh } \\
\text { Consemed }\end{array}$} \\
\hline & 11000 & kWh Boctic & $1 / 1,00$ & kwh Eoctic & & & \\
\hline Januery & 0.00 & 0.00 & 022 & 789 & 5.19 & 634 & 18.42 \\
\hline Fobruay & 0.00 & 0.00 & 022 & 6.78 & 4.69 & 456 & 16.04 \\
\hline March & 0.00 & 0.00 & 0.22 & 5.31 & 5.19 & 4.12 & 14.62 \\
\hline Apri & 0.00 & 0.00 & 022 & 211 & 5.02 & 323 & 1036 \\
\hline May & 029 & 5.18 & 022 & 0.00 & 5.19 & 264 & 1301 \\
\hline fron & 020 & 728 & 022 & 0.00 & 5.02 & 255 & 14.85 \\
\hline Jury & 029 & 9.37 & 0.22 & 0.00 & 5.19 & 2.64 & 17.19 \\
\hline August & 029 & 8.46 & 022 & 0.00 & 5.19 & 2.64 & 1629 \\
\hline Seprenber & 0.29 & 5.06 & 0.22 & 0.00 & 5.02 & 3.06 & 13.14 \\
\hline Octuber & 029 & 3.09 & 022 & 102 & 5.19 & 381 & 13.12 \\
\hline Norentiber & 0.00 & 0.00 & 0.22 . & 4.67 & 5.02 & 5.03 & 14.71 \\
\hline Decoribar & 0.00 & 0.00 & 022 & 723 & 5.19 & 6.54 & 18.96 \\
\hline TOTAm2 & & 38.45 & & 35.02 & 61.11 & 47.14 & 981.71 \\
\hline
\end{tabular}


OPTION 2: SEMLTRANSPARENT DOUBLE GLAZED PV ROOF .

STIEDATA

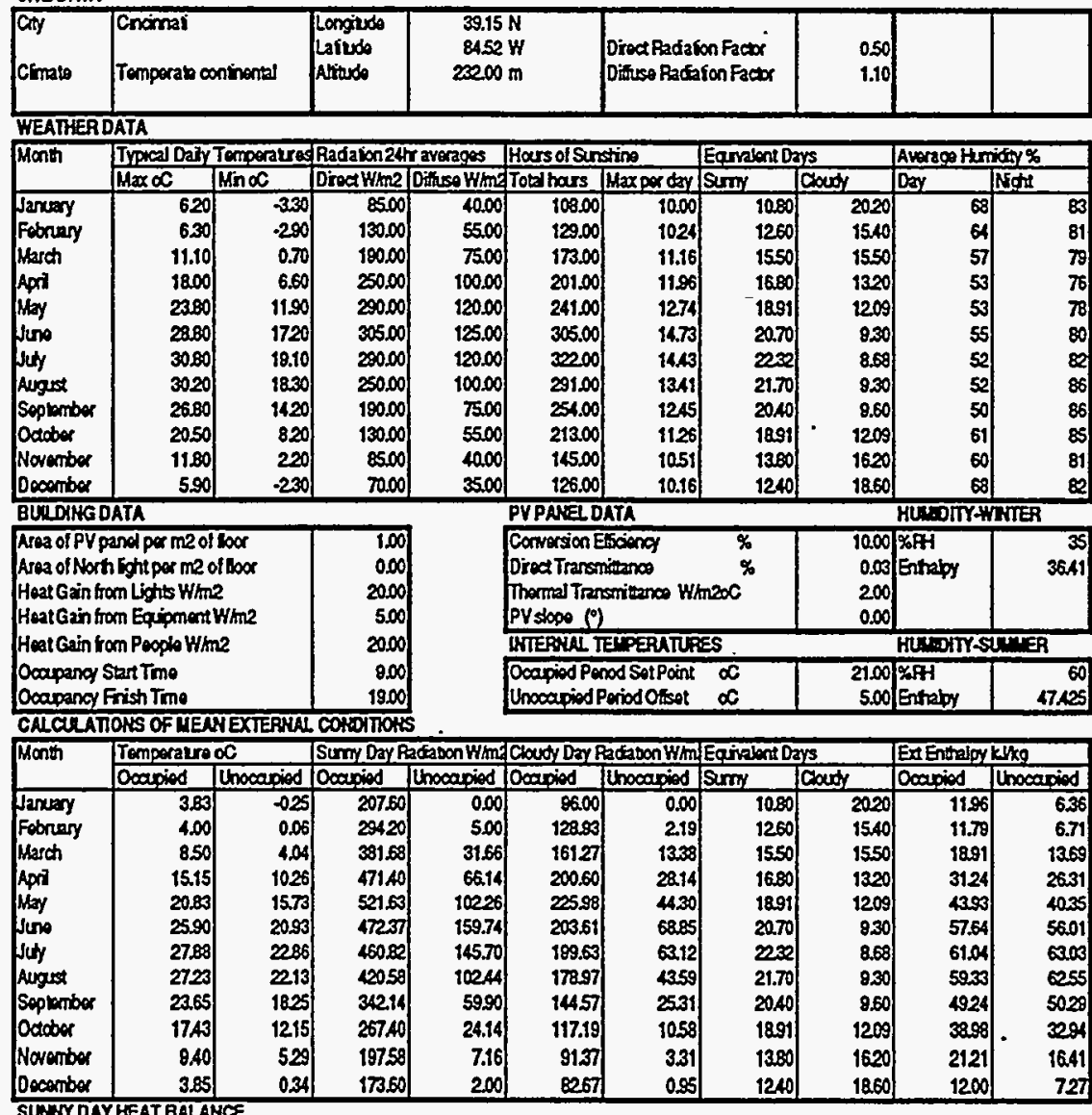

\begin{tabular}{|c|c|c|c|c|c|c|c|c|c|c|}
\hline \multirow[t]{2}{*}{ Manth } & \multicolumn{3}{|c|}{ Ocaloied Period W/m2 } & \multicolumn{3}{|c|}{ Unocanoied Period W/m2 } & \multicolumn{4}{|c|}{ 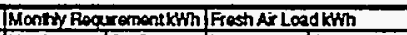 } \\
\hline & PV nat gain & Internals & Onoral & PVnotgain & internals & Derell & Heating & $\operatorname{cosing}$ & Heaing & $\operatorname{cosing}$ \\
\hline January & -16.85 & 2500 & 8.15 & -3249 & 450 & -27.89 & $\sqrt{23}$ & 088 & 5.07 & 0.00 \\
\hline Fabriary & .920 & 25.00 & 1580 & $-31,46$ & 450 & -26.86 & +76 & 199 & -596 & 0.00 \\
\hline March & 7.18 & 2500 & 3218 & -2125 & 450 & -16.75 & 3.63 & 499 & -521 & 0.00 \\
\hline Apri & 2804 & 25.00 & 53.04 & -590 & 450 & -140 & -0.33 & 891 & -1.67 & 0.00 \\
\hline May & 43.62 & 25.00 & 6202 & 3.07 & 450 & 757 & 0.00 & 1498 & 0.00 & 0.00 \\
\hline Jino & 49.62 & 25.00 & 74.62 & 6.66 . & 450] & 11.16 & 0.00 & 18.68 & 0.00 & 4.06 \\
\hline suty & 5260 & 25.00 & 7.60 & 6.00 & 450 & 10.50 & 0.00 & $20 \mathrm{EO}$ & 0.00 & 5.83 \\
\hline August & 47.90 & 25.00 & 7290 & 422 & 4.50 & 8.72 & 0.00 & 1847 & 0.00 & 4.96 \\
\hline Sapianter & 34.14 & 2500 & 59.14 & 155 & 4.50 & 6.05 & 0.00 & 13.79 & 0.00 & 0.71 \\
\hline Oactorer & 1539 & 25.00 & 40,39 & 5.66 & 450 & -1.16 & $-0,31$ & 764 & 0.00 & 0.00 \\
\hline November & 6.54 & 25.00 & 1846 & -2083 & 4.50 & -1633 & 3.15 & 255 & 4.03 & 0.00 \\
\hline Decomber & -19.67 & 25.00 & 5.33 & 31.16 & 450 & -26.66 & 4.63 & 0.66 & -581 & 0.00 \\
\hline
\end{tabular}

\begin{tabular}{|c|c|c|c|c|c|c|c|c|c|c|c|}
\hline \multirow[t]{2}{*}{ Month } & \multicolumn{3}{|c|}{ Dearoiod Period W/m2 } & \multicolumn{3}{|c|}{ Unocolongd Period W/m2 } & \multicolumn{2}{|c|}{ Monthy Ragroment kWh } & \multicolumn{3}{|c|}{ FreshAir LoadkWh } \\
\hline & Py matgain & Inomels & Iovera! & PVnotgin & Intemis & Ooverd & Heating & Cooling & Heaing & Cooin & \\
\hline January & .2626 & 4500 & 18.74 & 3248 & 450 & -27.99 & -7.92 & 3.79 & -9.48 & & 0.00 \\
\hline Februery & -23.13 & 40.00 & 1687 & -31.70 & 450 & -2720 & 5.86 & 260 & -728 & & 0.00 \\
\hline Warch & -1140 & 35.00 & 23.60 & -22.78 & 450 & -18.29 & 397 & 3.66 & $\$ 21$ & & 0.00 \\
\hline Aprit & 521 & 30.00 & 3521 & -9.10 & 450 & -4.60 & -0.85 & 465 & -131 & & 0.00 \\
\hline May & 18.70 & 25.00 & 43.70 & -122 & 4.50 & 268 & 0.00 & 5.74 & $0, \infty$ & 0 & 0.00 \\
\hline Juno & 26.96 & 25.00 & 51.96 & 3.66 & 450 & 8.16 & 0.00 & 590 & 0.00 & & 1.82 \\
\hline Juty & 30.58 & 25.00 & 5558 & 4.04 & 450 & 854 & 0.00 & 586 & 0.00 & & $22 \pi$ \\
\hline wigest & 27.54 & 2500 & 5254 & 259 & 4.50 & 7.09 & 0.00 & 5.81 & 0.00 & & 2.13 \\
\hline eplantber & $17 \mathrm{Ag}$ & 30.00 & 4719 & -137 & 450 & 3.13 & 0.00 & 4.98 & 0.00 & & 0.33 \\
\hline acoer & 273 & $\mathbf{3 5 . 0 0}$ & 37.73 & -680 & 450 & .230 & -0.39 & 456 & 0.00 & & 0.00 \\
\hline Novenber & .15 .50 & 40.00 & 2450 & -21.15 & 450 & -16.65 & -3.78 & 3.97 & 4.73 & & 0.00 \\
\hline Docomber & -2733 & 45.00 & 17.67 & -3125 & 4.50 & -26.75 & -697 & 329 & -8.72 & & 0.00 \\
\hline
\end{tabular}

OVERAL EKERG Y REOUAFEUENTS AND PROOUCTION

\begin{tabular}{|c|c|c|c|c|c|c|c|}
\hline \multirow[t]{2}{*}{ Mants } & \multicolumn{2}{|c|}{ Coding Consumpion } & \multicolumn{2}{|c|}{ Hea ing Consernotion } & \multirow{2}{*}{ Fans } & \multirow{2}{*}{$\begin{array}{l}\text { Lighting and } \\
\text { Powor kthl }\end{array}$} & \multirow{2}{*}{$\begin{array}{l}\text { Tobal kHh } \\
\text { Consumed }\end{array}$} \\
\hline & $1 / 100 p$ & KWh Eectic & $1 / 000$ & kth Escic & & & \\
\hline Januay & 0.00 & 0.00 & 0.22 & 5.93 & 5.68 & 6.68 & 1829 \\
\hline Fobreary & 0.00 & 0.00 & 022 & 530 & 5.13 & 4.69 & 15.13 \\
\hline March & 0.00 & 0.00 & 022 & 4.00 & 5.58 & 4.19 & 1387 \\
\hline Aprid & 029 & 3.87 & 022 & 0.52 & 550 & 321 & 1351 \\
\hline May & 029 & 5.92 & 022 & 0.00 & 5.68 & 264 & 1424 \\
\hline June & 0.28 & 8.70 & 0.22 & 0.00 & 5.50 & 255 & 16.75 \\
\hline fuly & 029 & 9.88 & 022 & 0.00 & 5.68 & 264 & 18.18 \\
\hline Aungest & 029 & 8.96 & 022 & 0.00 & 5.58 & 264 & 1728 \\
\hline Soptomber & 029 & 5.66 & 022 & 0.00 & 550 & 3.03 & 14.18 \\
\hline loctober & 028 & $3 A 9$ & 022 & 0.15 & 5.68 & 3.84 & 13.17 \\
\hline November & 0.00 & 0.00 & 022 & 3,49 & 5.50 & 4.98 & 1397 \\
\hline Decomber & 0.00 & 0.00 & 022 & 580 & 5.68 & 6.36 & 178.84 \\
\hline TOTAL/m2 & & 46,48 & & 25.61 & 66.92 & $47 A 2$ & 186.44 \\
\hline
\end{tabular}


OPTION 3:

FLAT INSULATED PV ROOF

\begin{tabular}{|c|c|c|c|c|c|c|c|c|c|c|}
\hline $\begin{array}{l}\text { Cay } \\
\text { Cinsto }\end{array}$ & $\begin{array}{l}\text { San Francisos } \\
\text { Temperam cos }\end{array}$ & stal wam & $\begin{array}{l}\text { Longute } \\
\text { Laitude } \\
\text { Alfitds }\end{array}$ & $\begin{array}{r}37.62 \\
12238 \\
200\end{array}$ & & $\begin{array}{l}\text { Oirect Radai } \\
\text { Dituso Rada }\end{array}$ & $\begin{array}{l}\text { on Factor } \\
\text { ion Factor }\end{array}$ & $\begin{array}{l}0.50 \\
1.10\end{array}$ & & \\
\hline WEATRA & DATA & & & & & & & & & \\
\hline Manth & Iypical Dayy & Terporalues & Radalion 24h & ravoraess & Hours of Suns & $\sin \theta$ & Equralont Da: & & Averzoe $\mathrm{Hm}$ & divy \\
\hline & $\operatorname{Max} \propto C$ & Un $\infty$ & DirodW/m2 & Dinse H/me & Totihars & Max per dy & $\mathbf{s} \mathrm{m}$ & Cousty & Day & Nont \\
\hline Januey & 1300 & 540 & 8500 & 40.00 & 154.00 & 1000 & 1540 & 1560 & 75 & 85 \\
\hline Fobruey & 14.70 & 6.30 & 130.00 & 5500 & 181.00 & 11.56 & 16.52 & 1418 & 71 & 85 \\
\hline March & 1640 & 720 & 190.00 & 7500 & 22000 & 1243 & 2108 & 9.52 & 66 & 8 \\
\hline Apri & 1790 & 840 & 250.00 & 100.00 & 297.00 & 13944] & 2130 & 8.70 & 66 & 85 \\
\hline May & 19.40 & 980 & 290.00 & 120.00 & 328.00 & 1490 & 2201 & 8.99 & 66 & 85 \\
\hline Jino & 2120 & 11,30 & 305.00 & 125.00 & 341.00 & 1536 & 220 & 7800 & 65 & 87 \\
\hline Juty & 2220 & 11.20 & 290.00 & 120.00 & 285.00 & 1420 & 20.77 & 1023 & 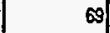 & 90 \\
\hline Angust & 2210 & 1210 & 250.00 & 100.00 & 275.00 & 13.65 & 20.15 & 10.85 & $\infty$ & 80) \\
\hline Septanter & 2320 & 1220 & 190.00 & 75.00 & 275.00 & 1273 & 21.60 & 840 & 66 & 88 \\
\hline Oatber & 2140 & 10.30 & 130.00 & 55.00 & 242.00 & 1131 & 21.39 & 9.51 & 65 & 86 \\
\hline Norember & 1780 & 7.70 & 85.00 & 40.00 & 198.00 & 10.00 & 18800 & 1020 & 68 & 84 \\
\hline December & 14.00 & 6.10 & 70.00 & 35.00 & 179.00 & 1033 & 17,36 & 13.64 & 74 & 86 \\
\hline BUTDING & IATA & & & & PVPAREID & ATA & & & सUसणTY & ETER \\
\hline $\begin{array}{l}\text { Area of PV } \\
\text { Area of Nor } \\
\text { Heat Gain } \\
\text { Heat Gain }\end{array}$ & 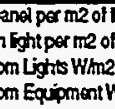 & $\begin{array}{l}\text { loor } \\
\text { fiboor } \\
2 \\
\text { Whan2 }\end{array}$ & $\begin{array}{r}1.00 \\
0.00 \\
20.00 \\
5.00\end{array}$ & & $\begin{array}{l}\text { Converson Es } \\
\text { Droct Transin } \\
\text { Thermal Trans } \\
\text { PV siopo ( ) }\end{array}$ & $\begin{array}{l}\text { ificioncy } \\
\text { sitanco } \\
\text { snitanco Whn }\end{array}$ & $\begin{array}{c}\% \\
\% \\
1200 c^{2}\end{array}$ & $\begin{array}{r}10.00 \\
0.00 \\
0.18 \\
0.00 \\
\end{array}$ & Entraby & $\begin{array}{r}35 \\
35,41\end{array}$ \\
\hline Hoat Gain 1 & on Peopio W/m & & 20.00 & & TRTERNAL TI & EUPERATURE & & & HULDOTYS & JMLER \\
\hline $\begin{array}{l}\text { Ocapany } \\
\text { Oocapancy }\end{array}$ & $\begin{array}{l}\text { Sart Tmo } \\
\text { Frish Tmo }\end{array}$ & & $\begin{array}{r}9.00 \\
19.00\end{array}$ & & $\begin{array}{l}\text { Oonpiod Peri } \\
\text { Unocosiod P }\end{array}$ & $\begin{array}{l}\text { iod SetPoint } \\
\text { Period Otsot }\end{array}$ & $\begin{array}{l}\infty \\
\infty\end{array}$ & $\begin{array}{r}21.00 \\
5.00\end{array}$ & $\begin{array}{l}\text { EAt } \\
\text { Entraby }\end{array}$ & $\begin{array}{r}60 \\
47.425 \\
\end{array}$ \\
\hline CALCILAT & IOHS OFUEA & NEXTERYAL & CoNolnoNs & & & & & & & \\
\hline Wonth & Temperaluo o & & Sumy Day R & afrabon Wine & Cloudy Day & adation W/m2 & Eqivalent DS $_{2}$ & & ExtEntedyyl & $\mathrm{c} k \mathrm{~kg}$ \\
\hline & Ocanoind & Uhocapiad & Oocapiod & Unocapiad & Oocapiod & Unocapiod & Sumy & County & Oocoind & Unocapoied \\
\hline Januay & 11.10 & 7.84 & 207.60 & 0.00 & 96.00 & 0.00 & 1540 & 1560 & 2301 & 2252 \\
\hline Fabruary & 1260 & 9.00 & 260.51 & 29.06 & 114.17 & 1274| & 1652 & 1148 & 30.55 & 25.18 \\
\hline Warch & 16.10 & 10.16 & 34275 & 59.46 & 144.82 & 25.13 & 21.08 & 8.92 & 3271 & 2753 \\
\hline Aprit & 15.53 & $11 A 5$ & 40448 & 113.24 & 172.12 & 48,48 & 2130 & 8.70 & 36,08 & $3 t .15$ \\
\hline Nay & 17.00 & 1289 & 446.11 & 15621 & 19328 & 67.67 & 2201 & 899 & 39.69 & 3484 \\
\hline June & 18.73 & 1448 & 453.11 & 173.49 & 19531 & 74.78 & 2220 & 780 & 4367 & 39.69 \\
\hline Juty & 19.53 & 1521 & 468.05 & 140.53 & $202 \pi$ & 6089 & 20.77 & 1023 & 47.62) & 4264 \\
\hline August & 19.60 & 1531 & 413.26 & 107.67| & 175.85 & 4582 & 20.15 & 10.85 & 4755 & 204 \\
\hline Sopminter & 2045 & 15.74 & 334.60 & 6528 & 141.38 & 27.58 & 21.60 & $8 A O$ & 48.59 & 4354 \\
\hline lacuber & 18.63 & 13887 & 26623 & 2498 & 116.67 & 10.95 & 21.39 & 8.61 & 4342 & 37.72 \\
\hline Noventort & 1520 & 10.85 & 207.60 & 0.00 & 9600 & 0.00 & 1980 & 1020 & 36.10 & 20.66 \\
\hline Docomber & 1203 & 8.64 & 171.08 & 3.80 & 81,47 & 181 & 1736 & 13.64 & 2994 & 2453 \\
\hline SLYRY & EAT BALAN & $\mathrm{NCE}$ & & & & & & & & \\
\hline Manth & Ocopied Peri & odw/nR & & UnocapiodP & griodW/m2 & & Monthy Requ & mernentkWh & IFresh Air Loa & $\mathrm{dkWh}$ \\
\hline & PVnotoin & Intands & Oaverl & PVnotgain & Intomis & Ovall & Hoaing & Cooling & Heating & $\operatorname{cosing}$ \\
\hline Januey & -021 & 45.00 & 44.79 & -147 & 450 & 3.03 & 0.00 & 755 & -248 & 0.00 \\
\hline Fabnesy & 0.46 & 45.00 & 45.46 & -1.04 & 450 & 3.46 & 0.00 & 831 & -186 & 0.00 \\
\hline March & 1.35 & 45.00 & 4635 & -0.60 & 450 & 390 & 0.00 & 10.92 & -150 & 0.00 \\
\hline Apri & 2.07) & 4500 & 47.07 & 0.04 & 450 & 454 & 0.00 & 1138 & -0.14 & 0.00 \\
\hline May & 265 & 4500 & 47.55 & 0.22 & 450 & 482 & 0.00 & 11.97 & 0.00 & 0.00 \\
\hline Jens & 302 & 45.00 & 4802 & 0.59 & 450 & 5.09 & 0.00 & 1224 & 0.00 & 0.00 \\
\hline Juty & 328 & 4500 & 4829 & $0<7$ & 450 & 497| & 0.00 & 1148 & 0.00 & 0.08 \\
\hline Augsos & 287 & 4500 & 4787 & 024 & 450 & 4.74 & 0.00 & 10.08 & 0.00 & 0.05 \\
\hline Septuribor & 243 & 45.00 & 47,43 & 0.00 & 450 & 450 & 0.00 & 11.60 & 0.00 & 0.48 \\
\hline loctober & 159 & 4500 & 4659 & -0.18 & 450 & 431 & 0.00 & 1125 & 0.00 & 0.00 \\
\hline Noventiber & 0.54 & 4500 & 4554 & -0.91 & 450 & 3.59 & 0.00 & 10.01 & -0.12 & 0.00 \\
\hline Docombar & $-0,32$ & 45.00 & 44.68 & $-1,30$ & 150 & 320 & 0.00 & 8.53 & -2.16 & 0.00 \\
\hline CLOWY & TYKEAT BALA & AHCE & & & & & & & & \\
\hline Manstr & Ocapiod Porio & odwhe & & Unocapiod P & eriodW/m2 & & $y \operatorname{Reg}$ & ntkWh & Frest Air Loa & dkWh \\
\hline & PV net gain & Intornals & Overall & PVnat gain & Intanals & Overal & Healing & Cooing & Heaing & Cooing \\
\hline January & -1.06 & 4500 & 4394 & $-\mid A 7$ & 4.50 & 3.03 & 0.00 & 752 & .252 & 0.00 \\
\hline bruey & -0.65 & 4500 & 44,35 & -1.16 & 450 & 334 & 0.00 & 5.63 & -129 & 0.00 \\
\hline Harch & -0.15 & 45.00 & 44.85 & -0.85 & 450 & 3.64 & 0.00 & 4.95 & -0.71 & 0.00 \\
\hline Apri' & 0.32 & 4500 & 4532 & -0.45 & 450 & 4.05 & 0.00 & 444 & -0.06 & 0.00 \\
\hline May & 0.74 & 4500 & 45.74 & -0.05 & 4.50 & 4.45 & 0.00 & 4.67. & 0.00 & 0.00 \\
\hline blone & 1.07 & 45.00 & 46.07 & 020 & 4.50 & 478 & 0.00 & 4.12 & 0.00 & 0.00 \\
\hline blty & 129 & 45.00 & 46.29 & 0.32 & 450 & 482 & 0.00 & 543 & 0.00 & 0.04 \\
\hline Augast & 1.08 & 45.00 & 46.08 & 0.22 & 450 & 4.72 & 0.00 & 5.72 & 0.00 & 0.03 \\
\hline parmber & 0.97 & 4500 & 4597 & 0.16 & 450 & 4.66) & 0.00 & 4A1 & 0.00 & 0.19 \\
\hline tober & 0.45 & 45.00 & 45.45 & -0.30 & 450 & 420 & 0.00 & 4.93 & 0.00 & 0.00 \\
\hline Nomber & -0.30 & 45.00 & 44.70 & -0.91 & 450 & 359 & 0.00 & 5.07 & -0.06 & 0.00 \\
\hline Deconber & -1.00 & 45.00 & 44.00 & $-1,31$ & 450 & 3.19 & 0.00 & 6.61 & -1.69 & 0.00 \\
\hline
\end{tabular}

OVERALL ERERG Y REOURELENTS AMD PRODUCTHON

\begin{tabular}{|c|c|c|c|c|c|c|c|}
\hline \multirow[t]{2}{*}{ Ment' } & \multicolumn{2}{|c|}{ Coding Consurnoion } & \multicolumn{2}{|c|}{ Hearing Consenotion } & \multirow{2}{*}{$\begin{array}{l}\text { Fans } \\
\text { kwh }\end{array}$} & \multirow{2}{*}{$\begin{array}{l}\text { Lighting and } \\
\text { Power kth }\end{array}$} & \multirow{2}{*}{$\begin{array}{l}\text { TotalkMh } \\
\text { Consumed }\end{array}$} \\
\hline & 1 kopp & the Eectic & $1 / \operatorname{cop}$ & kth Eloctic & & & \\
\hline Jaruery & 0.00 & 0.00 & 022 & 1.11 & 3.66 & 8.84 & 13.60 \\
\hline Februey & 0.00 & 0.00 & 022 & 0.70 & 3.30 & 7.98 & 11.58 \\
\hline March & 0.00 & 0.00 & 0.22 & 0.48 & 3.66 & 8.84 & 1298 \\
\hline Aprit & 020 & 452 & 022 & 0.04 & 3.54 & 8.55 & 16.65 \\
\hline May & 029 & 4.76 & 022 & 0.00 & 3.66 & 8.84 & 1725 \\
\hline Juno & 022 & 4.67 & 022 & 0.00 & 354 & 855 & 1676 \\
\hline Juty & 029 & 4.86 & 0.22 & 0.00 & 3.66 & 8.84 & 17.35 \\
\hline Augast & 029 & 4.79 & 022 & 0.00 & 3.65 & 8.84 & 1728 \\
\hline Sopmonber & 029 & 4.77 & 0.22 & 0.00 & 3.54 & 8.55 & 16.86 \\
\hline Octcoser & 020 & 4.62 & 022 & 0.00 & 3.66 & 884 & $17: 12$ \\
\hline Novernber & 029 & 4.31 & 022 & 0.04 & 3.54 & 8.55 & 16,4 \\
\hline Decomber & 0.00 & 0.00 & 022 & 0.86 & 3.66 & 8.84 & 1335 \\
\hline TOTAL $m 2$ & & 37.30 & & 324 & 43.06 & 104.03 & 187.63 \\
\hline
\end{tabular}


SIEDATA

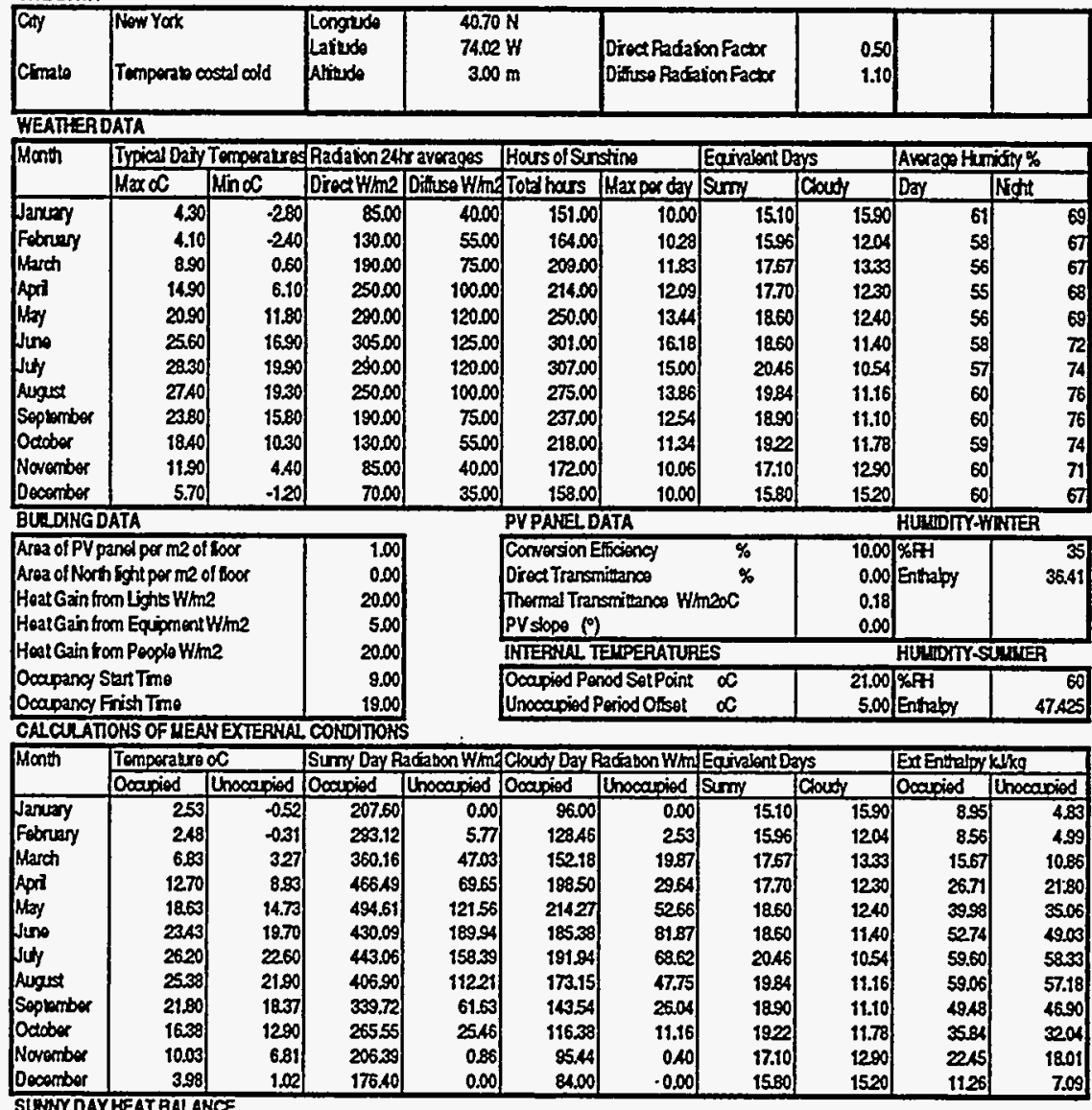

SUMTYDAYHEAT BALAKCE

\begin{tabular}{|c|c|c|c|c|c|c|c|c|c|c|}
\hline \multirow[t]{2}{*}{ Manth } & \multicolumn{3}{|c|}{ Ooapiod Period W/n2 } & \multicolumn{3}{|c|}{ Unocapiod Penod Whn? } & \multicolumn{4}{|c|}{ Montly Rearemenk kWh Frosti Ar Load kWh } \\
\hline & PVnotgain & Intomals & Overal & PVnotgin & Internals & Overal & Hoaing & Cooing & Hoaling & $\operatorname{cosing}$ \\
\hline Januay & -1.76 & 45.00 & 4324 & -297 & 450 & 153 & 0.00 & 685 & .795 & $\overline{0.00}$ \\
\hline ny & -1.12 & 45.00 & 43.88 & -280 & 450 & 1.61 & 0.00 & 736 & -854 & 0.00 \\
\hline Irch & 0.17 & 4500 & 45.17 & -184 & 450 & 256 & 0.00 & 8.62 & -7.04 & 0.00 \\
\hline Apri & 203 & 4500 & 4703 & -0.75 & 450 & 3.75 & 0.00 & 825 & 330 & 0.00 \\
\hline by & 3.31 & 45.00 & 4831 & -021 & 4.50 & 420 & 0.00 & 10.10 & 0.00 & 0.00 \\
\hline no & 3.60 & 45.00 & 48.69 & 0.60 & 450 & 5.10 & 0.00 & 1038 & 0.00 & 190 \\
\hline Juty & 429 & 4500 & 4929 & 0.59 & 450 & 500 & 0.00 & 1154 & 0.00 & 4.78 \\
\hline Auguse & 386 & 45.00 & 4886 & OA1 & 450 & 4.91 & 0.00 & 11.06 & 0.00 & $4 A 3$ \\
\hline planter & 2.71 & 45,00 & 47.71 & -0.01 & 450 & 449 & 0.00 & 1021 & 0.00 & 0.75 \\
\hline Octuber & 1.18 & 45.00 & 46.18 & -0.36 & 450 & 4.14 & 0.00 & 9.99 & .021 & 0.00 \\
\hline wamber & -042 & 45.00 & 458 & -1.65 & 450 & 285 & 0.00 & $83 i$ & -458 & 0.00 \\
\hline Decomber & -1.73 & 45.00 & 4327 & -270 & 450 & 1.80 & 0.00 & 724 & .7 .63 & 0.00 \\
\hline
\end{tabular}

CLOUDY DAY HEAT BALANCE

\begin{tabular}{|c|c|c|c|c|c|c|c|c|c|c|}
\hline \multirow[t]{2}{*}{ Manth } & \multicolumn{3}{|c|}{ Conpod PenodW/m2 } & \multicolumn{3}{|c|}{ Unosapood Poriod W/m2 } & \multicolumn{2}{|c|}{ Monthy Regurement kWh } & \multicolumn{2}{|c|}{ Figsh AirLoadkHh } \\
\hline & PVnetgain & Intomests & Oworall & PVnetgain & Inbmals & Oreral & Heaing & Cooling & Heatix & Cooling \\
\hline$\sqrt{\text { Jaxury }}$ & -260 & 4500 & 4240 & -297 & 450 & 153 & 0.00 & 708 & -838 & 0.00 \\
\hline Fobruary & -236 & 45,00 & 4264 & -202 & 450 & 158 & 0.00 & 5.40 & -6.44 & 0.00 \\
\hline March & -140 & 45.00 & 43.60 & -214 & 450 & 236 & 0.00 & 625 & -539 & 0.00 \\
\hline prii & 0.01 & 45.00 & 4501 & -1.05 & 4.50 & 3.45 & 0.00 & 6.13 & -222 & 0.00 \\
\hline by & 1.19 & 4500 & 46.19 & 0.17 & 4.50 & 4.67 & 0.00 & 654 & 0.00 & 0.00 \\
\hline Jino & 1.84 & 45.00 & 4684 & 029 & 450 & 4.79 & 0.00 & 6.30 & 0.00 & 1.16 \\
\hline luby & 239 & 45.00 & 47.39 & 0.36 & 450 & 486 & 0.00 & 5.71 & 0.00 & 246 \\
\hline August & 2.10 & 45.00 & 47.10 & 022 & 450 & 4.72 & 0.00 & 5.99 & 0.00 & 2.49 \\
\hline Soplanbor & 123 & 45.00 & 4623 & -0.19 & 450 & 431 & 0.00 & 580 & 0.00 & 0.44 \\
\hline Datober & 0.05 & 45.00 & 45.05 & -0.47 & 450 & 4.03 & 0.00 & 5.97 & -0.13 & 0.00 \\
\hline Voventber & -125 & 45.00 & 43.75 & -1.55 & 450 & 285 & 0.00 & 6.16 & 346 & 0.00 \\
\hline Decomber & -243 & 45.00 & 4257 & -270 & 450 & 180 & 0.00 & 6.25 & -734 & 0.00 \\
\hline
\end{tabular}

$$
\text { OVERALI ERERG YREQURELRENTS AND PRODUCTOH }
$$

\begin{tabular}{|c|c|c|c|c|c|c|c|}
\hline \multirow[t]{2}{*}{ Manth } & \multicolumn{2}{|c|}{ Coding Consumpion } & \multicolumn{2}{|c|}{ Heating Consumption } & \multirow{2}{*}{$\begin{array}{l}\text { Fans } \\
\text { kWh }\end{array}$} & \multirow{2}{*}{$\begin{array}{l}\text { Lighting and } \\
\text { Power kWh }\end{array}$} & \multirow{2}{*}{$\begin{array}{l}\text { Tow xph } \\
\text { Constrod }\end{array}$} \\
\hline & $1 / 00 p$ & KWh Bectic & $1 / \cos$ & kHh Eectic & & & \\
\hline Januay & 0.00 & 0.00 & 022 & 3.63 & 3.71 & 8.84 & 16.18 \\
\hline Fobruery & 0.00 & 0.00 & 022 & 3.33 & 3.25 & 7.98 & 14.66 \\
\hline March & 0.00 & 0.00 & 022 & 2.74 & 3.71 & 884 & 1520 \\
\hline Apri & 0.00 & 0.00 & 022 & 124 & 359 & 855 & 1338 \\
\hline May & 0.29 & 4.75 & 022 & 0.00 & 3.71 & 8.84 & 1730 \\
\hline Jeno & 029 & 559 & 022 & 0.00 & 359 & 855 & 17.73 \\
\hline Jety & 0.20 & 7.00 & 022 & 0.00 & 3.71 & 884 & 1954 \\
\hline Alegast & 029 & 6.85 & 022 & 0.00 & 3.71 & 884 & 1939 \\
\hline Sepmber & 029 & 491 & 022 & 0.00 & 359 & 855 & 17.05 \\
\hline Octaber & 029 & 456 & 022 & 0.08 & $3.7 t$ & 884 & 17.18 \\
\hline Novarmber & 0.00 & 0.00 & 022 & 1.79 & 359 & 855 & 1393 \\
\hline Docomber & 0.00 & 0.00 & 0.22 & 3.33 & 3.71 & 8.84 & 15.87 \\
\hline TOTALm2 & & 33.66 & & 16.14 & 43.66 & 104.03 & 197.49 \\
\hline
\end{tabular}


OPTION 3: FLAT INSULATED PV ROOF

\begin{tabular}{|c|c|c|c|c|c|c|c|c|c|c|}
\hline Coty & $\begin{array}{l}\text { Phoorix } \\
\text { Toctuperaty ar }\end{array}$ & & $\begin{array}{l}\text { Longitude } \\
\text { Laitude } \\
\text { Alitude }\end{array}$ & $\begin{array}{r}33.43 \\
112.02 \\
340.00\end{array}$ & & $\begin{array}{l}\text { Droct Padad } \\
\text { Dithuso Rade }\end{array}$ & $\begin{array}{l}\text { Son Factor } \\
\text { afon Factor }\end{array}$ & $\begin{array}{l}1.10 \\
0.90\end{array}$ & & \\
\hline WEATHER & JATA & & & & & & & & & \\
\hline Month & Typical Dary & Tomperatras & Radation 24h & tr averagas & Hours of Sun & $\sin \theta$ & Earialent Do & & Average Hum & indify $\%$ \\
\hline & $\operatorname{Max} \alpha$ & HnoC & DroctW/h2 & DitursoWhat & Toalhours & Maxper day & amy & Cound & Day & Hotst \\
\hline Januery & 1780 & 180 & 105.00 & 2500 & 26200 & 10.84 & 24.18 & 6.28 & 4 & 72 \\
\hline Fabruny & 20.10 & 380 & 150,00 & 3000 & 271.00 & 1241 & 2184 & 6.16 & 42 & 71 \\
\hline March & 2390 & 6.10 & 20500 & 3500 & 331.00 & 1256 & 2635 & 4.65 & 31 & 60 \\
\hline Apri & 2980 & 1020 & 26000 & 45.00 & 360,00 & 13,48 & 2670 & 3.30 & 26 & 50 \\
\hline May & 3380 & 1390 & 205.00 & 45.00 & 408,00 & 14.00 & 20.14 & 186 & 19 & 41 \\
\hline Jin & 3970 & 1860 & 305.00 & 50.00 & 411.00 & 1457 & 2820 & 180 & 17 & 37 \\
\hline Ity & 4030 & 2390 & 205.00 & 4500 & 390.00 & 14.30 & 2728 & 3.72 & 29 & 51 \\
\hline August & 38.70 & 2300 & 260.00 & 45.00 & 379.00 & 1422 & 26.66 & 434 & 35 & 60 \\
\hline Septerber & 3680 & 19.60 & 205.00 & 3500 & 354.00 & 13.11 & 27.00 & 3.00 & 2 & 66 \\
\hline Odtaber & 30.40 & 1260 & 150.00 & 30.00 & 323.00 & 11.98 & 2697 & 403 & 30 & 58 \\
\hline Novernber & 2320 & 5.80 & 105.00 & 25.00 & 282.00 & 10.93 & 2580 & 420 & 33 & 83 \\
\hline loscomber & 1890 & 280 & 90.00 & 25.00 & 260.00 & 10.75 & 24.18 & 6.82 & 42 & 70 \\
\hline BULDNG & ATA & & & & FV PANEL D & ATA & & & HURDITH & WWIER \\
\hline $\begin{array}{l}\text { Area of PV } \\
\text { Area of } N \text { ox } \\
\text { Heat Gain } \\
\text { Heat Gain }\end{array}$ & $\begin{array}{l}\text { hel per m2 of } \\
\text { onght per me o } \\
\text { on Lights H/m } \\
\text { om Eqipment }\end{array}$ & $\begin{array}{l}\text { Tleor } \\
\text { of foox } \\
\text { Wh } \\
\text { Whan2 }\end{array}$ & $\begin{array}{r}1.00 \\
0.00 \\
20.00 \\
5.00\end{array}$ & & $\begin{array}{l}\text { Conversion E } \\
\text { Droct Transm } \\
\text { Thermal Tran } \\
\text { py slope ( }{ }^{\circ}\end{array}$ & $\begin{array}{l}\text { Incioncy } \\
\text { nitancos } \\
\text { smitanco Wh }\end{array}$ & $\ln 2 x c^{\%}$ & $\begin{array}{r}10.00 \\
0.00 \\
0.18 \\
0.00 \\
\end{array}$ & Ontraby & $\begin{array}{r}35 \\
36.41\end{array}$ \\
\hline Heat Gain & on Peoplo W/h & & 20.00 & & IITERNAL T & EMPEAATUR & & & HULIITT-S & SUnER \\
\hline $\begin{array}{l}\text { Ocapang } \\
\text { Ocapangy }\end{array}$ & $\begin{array}{l}\text { Sart Tme } \\
\text { Frish Tme }\end{array}$ & & $\begin{array}{r}9.00 \\
19.00\end{array}$ & & $\begin{array}{l}\text { Ocapiod Per } \\
\text { Unocapiod P }\end{array}$ & $\begin{array}{l}\text { rod Sol Point } \\
\text { Poriod Otsol }\end{array}$ & $\begin{array}{l}\infty \\
\infty\end{array}$ & $\begin{array}{r}21.00 \\
5.00\end{array}$ & Fof & $\begin{array}{r}60 \\
47.425\end{array}$ \\
\hline CALCUA & IOHS OFUER & MEXTEPNAI & CONDITRK & & & & & & & \\
\hline Month & Tomperaure & & Sumy Day & Gdaton W/ing & Cloudy Day $R$ & Radaton $\% / m$ & Equalent D & & ExtEntfaloy & $\mathrm{k} / \mathrm{kg}$ \\
\hline & Ocaciod & Thocapiod & Tosopiad & Thoosoind & ocapied & Thocaoied & $\sin y$ & Coudy & Docupiod & Urocaniod \\
\hline Januay & $13 \mathrm{BO}$ & 6.94 & 305.66 & 1824 & 110.75 & 6.61 & 24.18 & 6.82 & 2584 & 18.43 \\
\hline Febreary & 1603 & 9.04 & 37136 & $\$ 89$ & 116.05 & 19.96 & 21.84 & 6.16 & 2956 & 2260 \\
\hline March & 18.45 & 1182 & 491.02 & 89.84 & 133.74 & 24,47 & 2635 & 4.65 & 31.89 & 26.13 \\
\hline Apri & 24.15 & 16.18 & 581.17 & 144.59 & 16020 & 3986 & 26.70 & 330 & 3782 & 3246 \\
\hline Hay & 2883 & 2030 & 625.65 & 17882 & 15427 & 44.09 & 20.14 & 186 & 4152 & 37.62 \\
\hline thos & 33.68 & 2506 & 626.58 & 204.73 & 164.67 & .5381 & 2820 & 180 & 47.87 & 45.49 \\
\hline Juty & 3620 & 29.17 & 61275 & 188.04 & 151.09 & 4636 & 2728 & 3.72 & 6.15 & 6382 \\
\hline August & 34.78 & 2305 & 55121 & 165.99 & 15194 & 45.76 & 26.66 & 434 & 65.41 & 66.63 \\
\hline Septanber & 3250 & 25.13 & $470 / 4$ & 10454 & 128.14 & 28,47 & 27.00 & 3.00 & 55.19 & 68.70 \\
\hline Octuber & 2595 & 18.32 & 384,76 & 54.31 & 12024 & 1697 & 2697 & 4.00 & 4331 & 40.03 \\
\hline Novertiber & 1885 & 1120 & 303.01 & 20.13 & 109.79 & 729 & 2580 & 420 & 31.51 & 30.54 \\
\hline Docontiber & 1488 & 7.89 & 271.19 & 1458 & 111.60 & 6.00 & 24.18 & 6.82 & 2738 & 2020 \\
\hline SUAN DA & YHEATBALA & WCE & & & & & & & & \\
\hline Manth & Docariod Pe & diodW/m2 & & Unocasiodf & PoriodWm2 & & Monty Rea & entkWh & 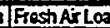 & $\mathrm{dowh}$ \\
\hline & PVnosoin & Intomals & Toveral & PVnotgain & Intemals & Overal & Hoaing & cooing & Heaing & $1 \operatorname{cosing}$ \\
\hline Janury & 1.01 & 45.00 & 46.01 & $-1 / 49$ & 450 & 3.01 & 0.00 & 12.14 & 485 & 0.00 \\
\hline Fabriay & 1.91 & 4500 & 46.91 & -0.7 & 450 & 3.73 & 0.00 & 1139 & -288 & 0.00 \\
\hline March & 3.43 & 4500 & 48.43 & -0.07 & 450 & 4.43 & 0.00 & 1440 & -220 & 0.00 \\
\hline Appi & 4.96 & 45.00 & 49.96 & 023 & 450 & 4.73 & 0.00 & 15.11 & 0.00 & 0.00 \\
\hline Maj & 6.14 & 4500 & 51.14 & 123 & 450 & 5.73 & 0.00 & 1724 & 0.00 & 0.00 \\
\hline Juno & 7.02 & 45.00 & 5202 & 138 & 450 & 588 & 0.00 & 16.99 & 0.00 & 024 \\
\hline Juty & 7.37 & 45.00 & 5237 & 1.99 & 450 & 6.49 & 0.00 & 16.77 & 0.00 & 824 \\
\hline August & 6.65 & 4500 & 51.65 & 1.02 & 450 & 6.12 & 0.00 & 16.05 & 0.00 & 921 \\
\hline Septentiber & 5.53 & 4500 & 50.63 & 0.63 & 450 & 5.13 & 0.00 & 15.61 & 0.00 & 4.18 \\
\hline loctaber & 3.80 & 4500 & 4880 & -0.07 & 450 & 4.43 & 0.00 & 1483 & 0.00 & 0.00 \\
\hline Noranber & 1.90 & 4500 & 46.90 & -0.68 & 450 & 3.82 & 0.00 & 1348 & -238 & 0.00 \\
\hline Decortber & 0.95 & 45.00 & 1595 & -133 & 450 & 3.17 & 0.00 & 1218 & -4.19 & 0.00 \\
\hline CLOWOD & T $\mathrm{BAL}$ & At & & & & & & & & \\
\hline Manth & Cocupiod Pex & riod W/m2 & & UnocapiodP & Whn & & Jineax & $t k W h$ & hATLLO & ad \\
\hline & PV net gain & In\$mals & Toveral & Pynot gain & Intema's & Toveral & Theaing & $\operatorname{cosing}$ & Hoating & Coosing \\
\hline Jaxky & -0.46 & 45.00 & 4454 & -158 & 450 & 292 & 0.00 & 3.32 & -137 & 0.00 \\
\hline Fobruary & -0.02 & 45.00 & 44.58 & -1.10 & 450 & 340 & 0.00 & 3.06 & .0 .81 & 0.00 \\
\hline Marath & 0.73 & 4500 & 45.73 & -0.57 & 4.50 & 3.93 & 0.00 & 238 & -0.40 & 0.00 \\
\hline Apti & 1.78 & 4500 & 46.78 & -0.12 & 450 & 4.38 & 0.00 & 1.75 & 0.00 & 0.00 \\
\hline May & 257 & 45.00 & 47.57 & 021 & 4.50 & 4.71 & 0.00 & 1.01 & 0.00 & 0.00 \\
\hline Juno & 3.53 & 4500 & 48.53 & 0.69 & 450 & 5.19 & 0.00 & 1.00 & 0.00 & 0.02 \\
\hline fuly & 3.88 & 45.00 & 48.88 & 0.92 & 450 & 5.12 & 0.00 & 2.10 & 0.00 & 1.12 \\
\hline Augus & 3.53 & 45.00 & 48.63 & 0.71 & 450 & 521) & 0.00 & $2 A B$ & 0.00 & 150 \\
\hline September & 3.04 & 45.00 & 48.04 & 0.06 & 450 & 456 & 0.00 & 1.68 & 0.00 & 0.46 \\
\hline Joctobor & 180 & 45.00 & 46.80 & -0.35 & 450 & 4.15 & 0.00 & 2.12 & 0.00 & 0.00 \\
\hline Nowomber & 0.44 & 4500 & 45.44 & -0.77 & 450 & 3.73 & 0.00 & 2.13 & -0.39 & 0.00 \\
\hline Decortber & -0.26 & 45.00 & 44.74 & $-1 A 0$ & 4.50 & 3.10 & 0.00 & 3.35 & -1.18 & 0.00 \\
\hline
\end{tabular}

OVERMII ERERGY REQUHEEMENIS AWD PRODU CTOH

\begin{tabular}{|c|c|c|c|c|c|c|c|}
\hline \multirow[t]{2}{*}{ Monts } & \multicolumn{2}{|c|}{ Cooing Consurmotion } & \multicolumn{2}{|c|}{ Hoaling Consumption } & \multirow{2}{*}{$\begin{array}{l}\text { Fans } \\
\text { kHh }\end{array}$} & \multirow{2}{*}{$\begin{array}{l}\text { Lighting and } \\
\text { Power kWh }\end{array}$} & \multirow{2}{*}{$\begin{array}{l}\text { Totikth } \\
\text { Consumed }\end{array}$} \\
\hline & 16000 & kWh Electic & 11000 & kith Eectic & & & \\
\hline Januery & 0.00 & 0.00 & 022 & 138 & 398 & 8.8 & 14.18 \\
\hline Fobruary & 029 & 4.13 & 022 & 0.82 & 358 & 7.98 & 16.51 \\
\hline March & 029 & 4.79 & 0.22 & 0.60 & 396 & 8.84 & 18.19 \\
\hline Apri & 020 & 481 & 022 & 0.00 & 3.83 & 8.55 & 1720 \\
\hline My & 029 & 521 & 0.22 & 0.00 & 396 & 884 & 1801 \\
\hline Juno & 020 & 521 & 022 & 0.00 & 3.83 & 855 & 17.60 \\
\hline Juty & 029 & 8.06 & 022 & 0.00 & 396 & 884 & 20.86 \\
\hline Augst & 020 & 8.34 & 022 & 0.00 & 3.96 & 8.84 & 23.14 \\
\hline Septomber & 020 & 625 & 0.22 & 0.00 & 383 & 855 & 18.64 \\
\hline loctobor & 029 & 484 & 022 & 0.00 & 3.96 & 8.84 & 97.64 \\
\hline Novertber & 029 & 4.46 & 0.22 & 0.61 & 383 & 855 & 17.46 \\
\hline Decomber & 0.00 & 0.00 & 0.22 & 1.19 & 3.96 & 8.8 & 1399 \\
\hline TOTAL/m2 & & 56.12 & & 4.61 & 46.65 & 104.00 & 211,41 \\
\hline
\end{tabular}




\section{OPTION 3: $\quad$ FLAT INSULATED PV ROOF}

STIE DATA

\begin{tabular}{|c|c|c|c|c|c|c|c|c|c|c|}
\hline $\begin{array}{l}\text { Coy } \\
\text { Cirnato }\end{array}$ & $\begin{array}{l}\text { Memi } \\
\text { sab-ropical }\end{array}$ & & $\begin{array}{l}\text { Longivide } \\
\text { Laitude } \\
\text { Arivide }\end{array}$ & $\begin{array}{l}2580 \\
8027 \\
200\end{array}$ & & $\begin{array}{l}\text { Direat Radal } \\
\text { Dithuse Rada }\end{array}$ & $\begin{array}{l}\text { lon Factor } \\
\text { afon Factor }\end{array}$ & $\begin{array}{l}0.50 \\
1.10\end{array}$ & & \\
\hline VEATHERI & JATA & & & & & & & & & \\
\hline Month & Typical oaly & Temperatures & Radaion 24 & raverages & Hours of Surs & $\sin \theta$ & Egrialont Da & ays & Average Hum & Difity \\
\hline & $\mathrm{H} \times \mathrm{OC}$ & $\operatorname{Lin} \alpha$ & Direct W/m2 & DitusoWhnt & Total hours & Maxper day & Sury & Cound & Day & Notis \\
\hline Janery & 2430 & 1440 & 150.00 & 65.00 & 239.00 & 1151 & 20.77 & 1023 & 5 & 84 \\
\hline Fabriary & 2500 & 1490 & 19500 & 80.00 & 233,00 & 11.72 & 19.88 & 8.12 & 56 & 84 \\
\hline March & 26.60 & 1620 & 205.00 & 8500 & 209.00 & 1222 & 2201 & 8.99 & 56 & 82 \\
\hline pri & 2810 & 1880 & 275.00 & 115.00 & 283.00 & 13.10 & 21.60 & 840 & 56 & 70 \\
\hline ky & 29.70 & 20.90 & 205.00 & 125.00 & 276.00 & 1349 & 20.46 & 1054 & 59 & 78 \\
\hline ino & 31.10 & 23.10 & 205.00 & 125.00 & 255.00 & 13.93 & 1830 & 1170 & 64 & 81 \\
\hline |Wy & 31.60 & 23.70 & 285.00 & 125.00 & 270.00 & 1382 & 19.53 & $11, A 7$ & 64 & 81 \\
\hline Woust & 3210 & 2380 & 275.00 & 115.00 & 261.00 & 13.16 & 19.84 & 11.16 & 63. & 83 \\
\hline Soplentor & 31.10 & 23.70 & 235.00 & 95.00 & 21200 & 11.58 & 17.70 & 1230 & 66 & 85 \\
\hline tober & 29.30 & 21.60 & 195.00 & 80.00 & 200.00 & 11.73 & 17.05 & 1395 & 64 & 65 \\
\hline torember & 2680 & 18.10 & 150.00 & 6500 & 224.00 & 11.31 & 19.80 & 1020 & 60 & 84 \\
\hline Decomber & 26.10 & 15.10 & 135.00 & 5500 & 221.00 & 11.14 & 19.84 & 11.16 & 60 & B5 \\
\hline BULDINGD & ATA & & & & PY PAKEI D & ATA & & & HUMTIY & DNTER \\
\hline $\begin{array}{l}\text { Area of PVP } \\
\text { Area of Nort } \\
\text { Heat Gain to } \\
\text { Heat Gain to }\end{array}$ & $\begin{array}{l}\text { and per m2 of } \\
\text { inght per me of } \\
\text { xn Lights W/me } \\
\text { m EquipmentV }\end{array}$ & $\begin{array}{l}\text { loox } \\
\text { Isoor } \\
2 \\
\text { W/m2 }\end{array}$ & $\begin{array}{r}1.00 \\
0.00 \\
20.00 \\
5.00\end{array}$ & i & $\begin{array}{l}\text { Conversion Ef } \\
\text { Direct Transm } \\
\text { Thermal Tran } \\
\text { PV sicpo ( }\end{array}$ & $\begin{array}{l}\text { Ificioncy } \\
\text { nittanco } \\
\text { smitance Wh }\end{array}$ & $m=c^{\%}$ & $\begin{array}{r}10.00 \\
0.00 \\
0.18 \\
0.00\end{array}$ & $\begin{array}{l}\text { EFH } \\
\text { Entralpy }\end{array}$ & $\begin{array}{r}35 \\
36.41\end{array}$ \\
\hline Heat Gainto & m Poopio W/h & & 20.00 & & INETHAL TE & EUPERATUR & & & HUDTTYS & ThUER \\
\hline $\begin{array}{l}\text { Oocupaney } 5 \\
\text { Ocopancy } f\end{array}$ & Tht Tme & & $\begin{array}{r}8.00 \\
19.00\end{array}$ & & $\begin{array}{l}\text { Ocopiod Par } \\
\text { Unocapiod P }\end{array}$ & $\begin{array}{l}\text { Hod Sot Point } \\
\text { oriod Otset }\end{array}$ & $\begin{array}{l}\alpha \\
\alpha C\end{array}$ & $\begin{array}{r}21.00 \\
5.00\end{array}$ & $\begin{array}{l}\text { \%RH } \\
\text { Entraby }\end{array}$ & $\begin{array}{r}60 \\
47 A 25 \\
\end{array}$ \\
\hline CALCULAT & OUS OF UEA & AEXIERMAL & COHOITOHS & & & & & & & \\
\hline Manth & Temperauroo & & Surm Day R & agsion W/RL & Cloudy Day 8 & adation Wh/ & Equalont Da & ays & ExtErtialpy $k$ & $\mathrm{k} / \mathrm{kg}$ \\
\hline & Ocapiod & thocapiod & Coapiod & Uhocapiod & Coapiod & Unocariod & siny & Coundy & Ooaviod & Thoocaid \\
\hline lanery & 21.83 & 1758 & 30555 & 3289 & 13557 & 1459 & 20.7 & 1023 & 49.08 & 47.57 \\
\hline Ebruary & 2248 & 18.15 & 379.85 & 46.68 & 103.82 & 20.13 & 19.88 & 8.12 & 4932 & 4923 \\
\hline arch & 2400 & 1954 & 435.95 & 69.18 & 186.55 & 29.50 & 2201 & 8.99 & 5328 & 5264 \\
\hline ni & 25.78 & 21.78 & 483.60 & 107.15 & 210.56 & 46.67 & 21.50 & 840 & 57.88 & 5821 \\
\hline May & 2750 & 23.73 & 507.05 & 12630 & 20230 & 5543 & 20.46 & 10.54 & 6443 & 6382 \\
\hline Juno & 29.10 & 2567 & 490.87 & 137.95 & 21520 & 60.50 & 1830 & 11.70 & 7243 & 71.86 \\
\hline uy & 29.63 & 2524 & 494.76 & 135.17| & 217.00 & 5929 & 19.53 & $11 A 7$ & 74.06 & 7380 \\
\hline Augr & 30.03 & 26.47 & 481.63 & 100.55 & 209.80 & 4728 & $19: 84$ & 11.16 & 74.60 & 75.78 \\
\hline oplember & 2925 & 2608 & 444.84 & 2083 & 190.36 & 2629 & 17.70 & 1230 & 7426 & 7558 \\
\hline & 2738 & 24.08 & 379.53 & 46.91 & 163.68 & 2023 & 17.05 & 13.95 & 67.19 & 68.60 \\
\hline & 24.63 & 2090 & 310.79 & 29.15 & 13789 & 1293 & 19.80 & 1020 & 57,00 & 57.67 \\
\hline Decomber & 23.35 & 18.64 & 275.78 & 2244 & 118.50 & 9.64 & $10: 34$ & 11.16 & 5356 & 51.08 \\
\hline
\end{tabular}

SUWHY DAYHEAT BALAKCE

\begin{tabular}{|c|c|c|c|c|c|c|c|c|c|c|}
\hline \multirow[t]{2}{*}{ Manth } & \multicolumn{3}{|c|}{ Ocapied Period W/m2 } & \multicolumn{3}{|c|}{ Unocospod Pexiod Whan2 } & \multicolumn{4}{|c|}{ Monthy RequsementkWh Frest ÁrLoadkwh } \\
\hline & PVnotoin & Intomals & Ooveral & PVnotgin & Internels & Geral & Heaing & $\cos i n$ & Healing & Cooling \\
\hline Jantary & 246 & 4500 & 4746 & -0.18 & 450 & 4.31 & 0.00 & 11.11 & 0.00 & $\overline{0.66}$ \\
\hline & 3.14 & 45.00 & 48.14 & 0.02 & 450 & 4.52 & 0.00 & 10.83 & 0.00 & 0.72 \\
\hline March & 38 & 4500 & 4884 & 0.17 & 4.50 & 4.67 & 0.00 & 1219 & 0.00 & 24 \\
\hline $\operatorname{sen}$ & 452 & 45.00 & 49.52 & 050 & 450 & 5.00 & 0.00 & 1221 & 0.00 & 434 \\
\hline Nay & 5.00 & 45.00 & 50.00 & 0.85 & 450 & 5.35 & 0.00 & 11.76 & 0.00 & 6.68 \\
\hline Jine & 5.17 & 4500 & 50.17 & 0.98 & 4.50 & 5.48 & 0.00 & 10.59 & 0.00 & 8.7 \\
\hline Judy & 529 & 4500 & 5028 & 106 & 450 & 5.56 & 0.00 & 1134 & 0.00 & 8.99 \\
\hline August & 527] & 45.00 & 5027 & 0.90 & 450 & 5.40 & 0.00 & $11 A 7$ & 0.00 & 1035 \\
\hline Soptentiber & 4.85 & 45.00 & 49.85 & $0.4 \theta$ & 450 & 4.99 & 0.00 & 10.05 & 0.00 & 9.12 \\
\hline Odaber & 4.02 & 4500 & 49.02 & 0.37 & 450 & 489 & 0.00 & 951 & 0.00 & 6.47 \\
\hline & 300 & 45.00 & 4800 & 0.02 & 450 & 452 & 0.00 & 10.76 & 0.00 & 3.64 \\
\hline 000 & 251 & 45.00 & 47.51 & -0.08 & 450 & 4.42 & 0.00 & 10.65 & 0.00 & 234 \\
\hline
\end{tabular}

CLOUDY DAY HEAT BALANCE

\begin{tabular}{|c|c|c|c|c|c|c|c|c|c|c|}
\hline \multirow[t]{2}{*}{ Mants } & \multicolumn{3}{|c|}{ Ocapied Period W/m2 } & \multicolumn{3}{|c|}{ Unocolpied PeriodW/m2 } & \multicolumn{4}{|c|}{ Monh Y RoguirementkWh F Festi Ar LaadkWh } \\
\hline & PVnetgin If & Inemals & Oraral & PVnotgain & Inbonats & Overal & Hoating & Coofing & Heaing & Cooting \\
\hline January & 1.17 & 45.00 & 46.17 & -0.05 & 450 & $\overline{4.45}$ & 0.00 & 5.36 & 0.00 & 0.33 \\
\hline Fob & 150 & 4500 & 46.50 & -0.05 & 450 & 4.44 & 0.00 & 428 & 0.00 & OSA \\
\hline March & 1.95 & 45.00 & 46.55 & 0.05 & 450 & 455 & 0.00 & 4.79 & 0.00 & 1.00 \\
\hline Apri & 245 & 45.00 & 47A5 & 0.19 & 450 & 4.69 & 0.00 & 454 & 0.00 & 1.69 \\
\hline May & 2.85 & 45.00 & 47.BS & 0.31 & 450 & 4.81 & 0.00 & 5.75 & 0.00 & 3.44 \\
\hline no & 3.09 & 45,00 & 4809 & 0.40 & 450 & 4.90 & 0.00 & 643 & 0.00 & 5.22 \\
\hline Jut & 3.19 & 45.00 & 48.19 & 0.49 & 450 & 4.99 & 0.00 & 633 & 0.00 & 5.86 \\
\hline August & 321 & 4500 & 4821 & 0.44 & 450 & 4.94 & 0.00 & 6.15 & 0.00 & 58 \\
\hline Septriber & 2.92 & 4500 & 47.92 & 022 & 450 & 4.72 & 0.00 & 6.71 & 0.00 & . \\
\hline Octuber & 238 & 45.00 & 4738 & 0.11 & 450 & 4.61 & 0.00 & 751 & 0.00 & 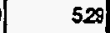 \\
\hline Norember & 1.69 & 45.00 & 46.69 & -0.01 & 450 & 4.49 & 0.00 & 540 & 0.00 & 18 \\
\hline Decembor & 132 & 45.00 & 4632 & -0.05 & 450 & 445 & 0.00 & 5.86 & 0.00 & 131 \\
\hline
\end{tabular}

OVERALI ENERGY FEOURELENTS AND PRODUCTIOH

\begin{tabular}{|c|c|c|c|c|c|c|c|}
\hline \multirow[t]{2}{*}{ Month } & \multicolumn{2}{|c|}{ Cooling Consumpion } & \multicolumn{2}{|c|}{ Heating Consmption } & \multirow{2}{*}{$\begin{array}{l}\text { Fans } \\
\text { kwh }\end{array}$} & \multirow{2}{*}{$\begin{array}{l}\text { Lighting and } \\
\text { Power kWh }\end{array}$} & \multirow{2}{*}{$\begin{array}{l}\text { Tobal kWh } \\
\text { Consemod }\end{array}$} \\
\hline & $1100 p$ & kwh Eectic & 11000 & kWh Bectic & & & \\
\hline Januey & 029 & 4.99 & 0.22 & 0,00 & 382 & 8.84 & 17.64 \\
\hline Fobruary & 0.29 & 4.61 & 022 & 0.00 & 3.45 & 7.98 & 16.04 \\
\hline March & 0.29 & 584 & 022 & 0.00 & 382 & $8 B 4$ & 18.49 \\
\hline Apri & 0.29 & 650 & 022 & 0.00 & 3.70 & 8.55 & 18.75 \\
\hline$M_{a y}$ & 029 & 7.90 & 0.22 & 0.00 & 3.82 & 8.84 & 20.55 \\
\hline Juno & 0.28 & 8.98 & 022 & 0.00 & 3.70 & 8.55 & 2122 \\
\hline Juts & 0.20 & 958 & 022 & 0.00 & 3.82 & 8.84 & 22.23 \\
\hline Augus: & 0.29 & 0.66 & 0.22 & 0.00 & 3.82 & 8.84 & 2231 \\
\hline Seprember & 0.20 & 921 & 022 & 0.00 & 3.70 & 855 & 21.45 \\
\hline Octaber & 020 & 822 & 0.22 & 0.00 & 3802 & 8.84 & 20.88 \\
\hline November & 029 & 6.19 & 0.22 & 0.00 & 3.70 & 8.55 & 18.44 \\
\hline Deconter & 0.29 & 5.76 & 022 & 0.00 & 3.82 & 884 & 18.42 \\
\hline TOTALm2 & & 8743 & & 0.00 & 4499 & 104.03 & 235,4 \\
\hline
\end{tabular}


BUILOING ENERGY BALANCE CALCULATIONS

OPTION 3: $\quad$ FLATINSULATED PV ROOF

STEDATA

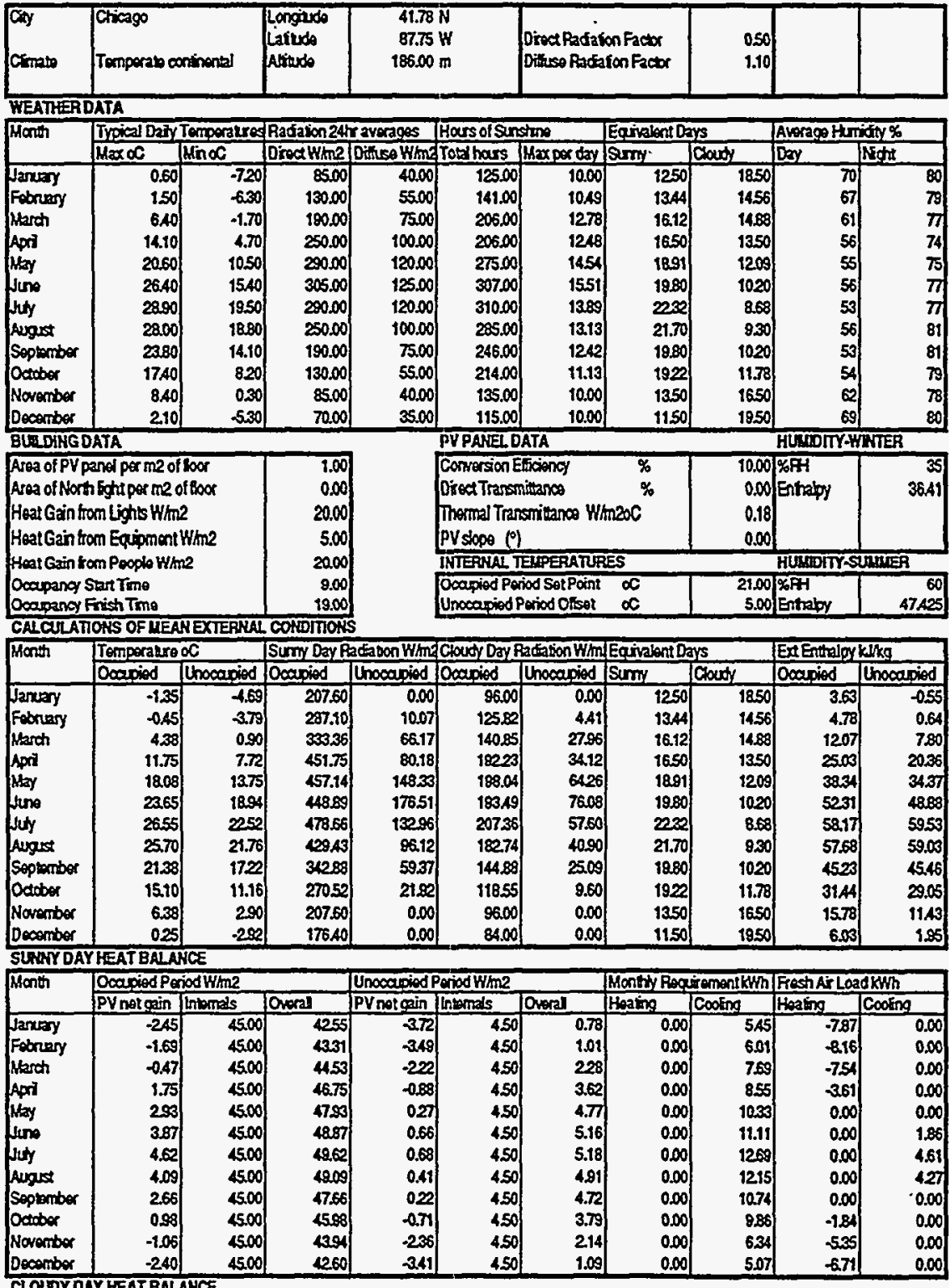

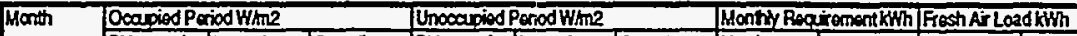

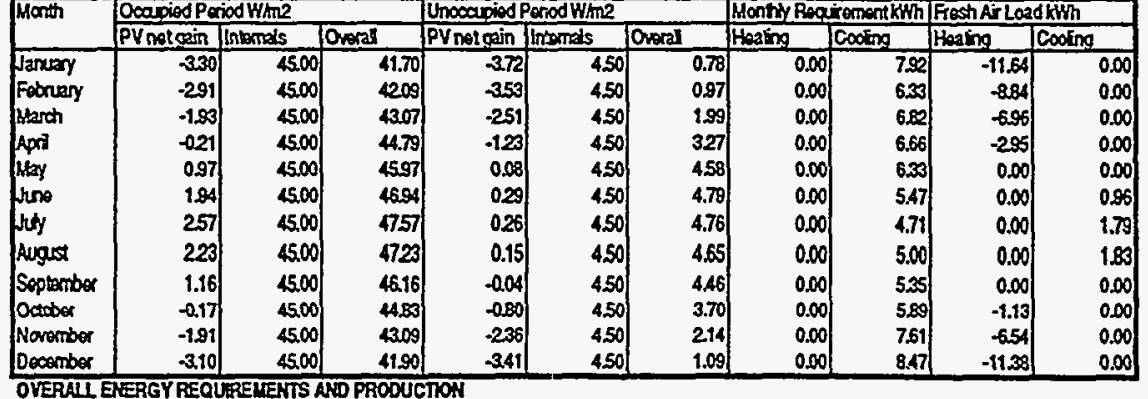

OVERALI ERERGY REQLEREIEATIS AND PRODUCTOH

\begin{tabular}{|c|c|c|c|c|c|c|c|}
\hline \multirow[t]{2}{*}{ Manth } & \multicolumn{2}{|c|}{ Coding Consumpion } & \multicolumn{2}{|c|}{ Heating Consunotion } & \multirow{2}{*}{$\begin{array}{l}\text { Fans } \\
\text { kHh }\end{array}$} & \multirow{2}{*}{$\begin{array}{l}\text { lighing and } \\
\text { Poworkwh }\end{array}$} & \multirow{2}{*}{$\begin{array}{l}\text { Total kth } \\
\text { Consumed }\end{array}$} \\
\hline & $1 k 000$ & kWhEsctic & 1600 & Krh Eectic & & & \\
\hline Januay & 0.00 & 0.00 & 022 & 4.34 & 3.72 & 8.84 & 16.89 \\
\hline breary & 0.00 & 0.00 & 022 & 3.78 & 336 & 798 & 15.12 \\
\hline ch & 0.00 & 0.00 & 0.22 & 322 & 3.72 & 884 & 15.78 \\
\hline$x$ & 0.00 & 0.00 & 022 & 1.46 & 3.60 & 8.55 & 13.61 \\
\hline May & 022 & 4.76 & 022 & 0.00 & 3.72 & 884 & 1732 \\
\hline Juno & 029 & 5.54 & 0.22 & 0.00 & 3.60 & 855 & 17.69 \\
\hline $\mathrm{J}(\mathrm{h})$ & 029 & 6.80 & 022 & 0.00 & 3.72 & 8.84 & 19.35 \\
\hline 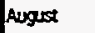 & 0.29 & 6.64 & 022 & 0.00 & 3.72 & 8.84 & 1920 \\
\hline mber & 028 & 460 & 022 & 0.00 & 3.60 & 855 & 1675 \\
\hline bor & 0.29 & 450 & 022 & 0.66 & 3.72 & 884 & 17.72 \\
\hline$\pi$ & 0.00 & 0.00 & 022 & 2.64 & 3.60 & 855 & 14.79 \\
\hline December & 0.00 & 0.00 & 0.22 & 4.02 & 3.72 & 8.84 & 16.58 \\
\hline TOTAL/A2 & & 3284 & & 20.11 & 43.83 & 104.03 & 2008 \\
\hline
\end{tabular}


STTE DATA

\begin{tabular}{|c|c|c|c|c|c|c|c|c|c|c|}
\hline $\begin{array}{l}\text { aty } \\
\text { Cirnato }\end{array}$ & $\begin{array}{l}\text { Cricrinal } \\
\text { Ternperab ox }\end{array}$ & minents & $\begin{array}{l}\text { Longrudo } \\
\text { Laitude } \\
\text { Altudedo }\end{array}$ & $\begin{array}{r}39.15 \\
84.52 \\
23200\end{array}$ & & $\begin{array}{l}\text { Oroct Radatio } \\
\text { Dituso Radai }\end{array}$ & $\begin{array}{l}\text { on Factor } \\
\text { ion Factor }\end{array}$ & $\begin{array}{l}0.50 \\
1.10\end{array}$ & & \\
\hline WEATHER & ATA & & & & & & & & & \\
\hline Month & Troical Dahy & Temperatres & Radaron 24h & raverages & Hars of Sur & sine & Equiplont $D_{c}$ & & Averes $\mathrm{H}$ m & idity \\
\hline & 수 & $\mathrm{Mn}_{\mathrm{n}}$ & Dreat W/m2 & DidisoW/m & Toth hars & Maxpex day & Sury & Coudy & Day & Ning \\
\hline Januxy & 620 & 330 & 8500 & 40.00 & 10800 & 10.00 & 1080 & 2020 & 6 & 83 \\
\hline Fobruary & 6.30 & -290 & 130.00 & 5500 & 129.00 & 1024 & 1260 & 1540 & 64 & 81 \\
\hline March & 11.10 & 0.70 & 19000 & 7500 & 173.00 & 11.16 & 1550 & 1550 & 57 & 79 \\
\hline Apri & 1800 & 6.60 & 250.00 & 100.00 & 20100 & 11.96 & 1680 & 1320 & 53 & 76 \\
\hline May & 2360 & 1190 & 20000 & 120.00 & 24100 & 1274 & 18.91 & 1200 & 53 & 78 \\
\hline Jino & 2880 & 1720 & 305.00 & 12500 & 305.00 & 14.73 & 20.70 & 930 & 5 & 80 \\
\hline Juty & 3080 & 19.10 & 20000 & 120.00 & 32000 & 14,43 & 2232 & 8.68 & 52 & 82 \\
\hline Augrst & 3020 & 1830 & 250.00 & 100.00 & 291.00 & 13.41 & 21.70 & 930 & 52 & 86 \\
\hline Seplember & 2680 & 1420 & 190.00 & 7500 & 254.00 & 1245 & 20.40 & 9.60 & 50 & 85 \\
\hline October & 20.50 & 820 & 130.00 & 55.00 & 213.00 & 1126 & 1891 & 1209 & 61 & es \\
\hline Novernbor & 11.80 & 220 & 8500 & 40.00 & 145.00 & 1051 & 1380 & 1620 & 60 & 81 \\
\hline Decombor & 5.90 & -230 & 70.00 & 35.00 & 126.00 & 10.16 & 1240 & 1860 & 68 & 82 \\
\hline BULDNGD & ATA & & & & PYPAKELD & ATA & & & KuMmory & INTER \\
\hline $\begin{array}{l}\text { Area of PVP } \\
\text { Area of Nort } \\
\text { Heat Grin if } \\
\text { Heat Gain th }\end{array}$ & $\begin{array}{l}\text { and per m2 of } \\
\text { a fight por me of } \\
\text { on Lights Whe } \\
\text { on Eqigment }\end{array}$ & $\begin{array}{l}\text { loor } \\
\text { of foor } \\
2 \\
\text { Whan }\end{array}$ & $\begin{array}{r}1.00 \\
0.00 \\
20.00 \\
5.00\end{array}$ & & $\begin{array}{l}\text { Corwersion } \\
\text { Direct Transm } \\
\text { Thermal Tran } \\
\text { PV slope (") }\end{array}$ & $\begin{array}{l}\text { difioncy } \\
\text { nitancos } \\
\text { smitanco Who }\end{array}$ & $\begin{array}{c}\% \\
\% \\
n\end{array}$ & \begin{tabular}{r|}
10.00 \\
0.00 \\
0.18 \\
0.00
\end{tabular} & Ertrapy & $\begin{array}{r}35 \\
354 t\end{array}$ \\
\hline Heal Guin $\mathrm{f}$ & m People Whin & & 20.00 & & INTERHAL TI & EXPEFATUR & & & HLUOTYSU & MUIER \\
\hline $\begin{array}{l}\text { Oocupaney } \\
\text { Ooapaney }\end{array}$ & $\begin{array}{l}\text { Tisht Tme } \\
\text { Tishe }\end{array}$ & & $\begin{array}{r}9.00 \\
18.00\end{array}$ & & $\begin{array}{l}\text { Ooapiod Pen } \\
\text { Unocoupiod P }\end{array}$ & $\begin{array}{l}\text { iod Sot Point } \\
\text { oriod Orsol }\end{array}$ & $\begin{array}{l}\infty \\
\infty\end{array}$ & $\begin{array}{r}21.00 \\
5.00\end{array}$ & $\begin{array}{l}\text { FFH } \\
\text { Entrapy }\end{array}$ & $\begin{array}{r}60 \\
47 A 25 \\
\end{array}$ \\
\hline CALCUAT & OWS OF MEA & REXTERILAL & CONOITOKS & & & & & & & \\
\hline Manth & Temperatroo & & Surm Day R & 3Gation H/m & Cloudy Day $R$ & 2adoon Winl & Equalentos & & Extentalgrk & $\mathrm{k} / \mathrm{kg}$ \\
\hline & Ooxoied & Urocapiod & Oonoind & Uhocapiod & Oocopiod & Unocapied & Surm & Couty & Oxapind & Inocariod \\
\hline Janeay & 3.83 & -025 & 207.60 & 0.00 & 8500 & 000 & 1080 & 20.20 & 11.95 & 636 \\
\hline Fobruary & 4.00 & 0.06 & 29420 & $5 . \infty$ & 128.93 & 2.19 & 1260 & 1540 & 11.79 & 6.71 \\
\hline March & 8.50 & 4.04 & 381.68 & 31.66 & 16127 & 1338 & 1550 & 15.50 & 1891 & 1369 \\
\hline Apri & 15.15 & 1026 & 47140 & 66.14 & 200.60 & 28.14 & 1680 & 1320 & 3124 & 2531 \\
\hline May & 20.83 & 15.73 & 521.63 & 10226 & 225.88 & 4430 & 1891 & 1209 & 4393 & 40.35 \\
\hline $\operatorname{Jin} \theta$ & 25.90 & 20.93 & 47237 & 159.74 & 203.61 & 68.25 & 20.70 & 9.30 & 5764 & 5601 \\
\hline Jut & 27.88 & 2286 & 46082 & 145.70 & 189.53 & 63.12 & 2230 & 8.58 & 6104 & 6303 \\
\hline August & 2722 & 2213 & 420.58 & $102 \mathrm{AS}$ & 178.97 & 4350 & 21.70 & 830 & 5933 & 6255 \\
\hline Soptenber & 23.65 & 1825 & 342.14 & 59.90 & 14.57 & 2531 & 20.40 & 9.60 & 4924 & 50.29 \\
\hline Detaber & 17,43 & 1215 & 267.40 & 24.14 & 197.19 & 1058 & 18.91 & 1209 & 3398 & 3294 \\
\hline Norember & 9.40 & 520 & 19758 & 7.16 & 9137| & 331 & 13800 & 1620 & 2121 & 16.49 \\
\hline Docombor & 3.85 & 0.34 & 173.60 & 2.00 & 82.67| & 0.55 & 1240 & 18.50 & 1200 & 727 \\
\hline
\end{tabular}

SLWWYDAYHEAT BALAYCE

\begin{tabular}{|c|c|c|c|c|c|c|c|c|c|c|}
\hline \multirow[t]{2}{*}{ Manth } & \multicolumn{3}{|c|}{ Ooapiod P uriod Whin2 } & \multicolumn{3}{|c|}{ Unocapied PeriodW/m2 } & \multicolumn{4}{|c|}{ Monfty ReairanontkWh Fresh Air Loadkith } \\
\hline & PVnetgain & Intemals & Overall & PVnatgain & Intema's & Overel & Hosing & Cooting & Healing & Cooling \\
\hline Januery & -1.52 & 4500 & 4348 & -282 & 450 & 158 & 0.00 & 493 & 507 & 0.00 \\
\hline Fab & -0.84 & 4500 & 44.16 & -283 & 450 & 1.67 & 0.00 & 586 & 596 & 0.00 \\
\hline Narch & 0.64 & 45.00 & 45.64 & -191 & 450 & 259 & 0.00 & 7.63 & 521 & 0.00 \\
\hline Apri & 251 & 4500 & 4751 & -0.53 & 450 & 3.97 & 0.00 & 8.91 & -1.67 & 0.00 \\
\hline Way & 3.91 & 4500 & 4891: & 027 & 450 & 4.77 & 0.00 & 1051 & 0.00 & 0.00 \\
\hline June & $4 / 45$ & 4500 & $49 / 5$ & 0.58 & 450 & 5.08 & 0.00 & 11.71 & 0.00 & 4.06 \\
\hline Juty & 4.72 & 4500 & 49.72 & 0.54 & 450 & 5.04 & 0.00 & 1267 & 0.00 & 5.83 \\
\hline August & 4.30 & 4500 & 4930 & 038 & 450 & 488 & 0.00 & 1218 & 0.00 & 496 \\
\hline Seplenber & 3.06 & 4500 & 48.06 & 0.14 & 450 & 4.64 & 0.00 & 11.13 & 0.00 & 0.71 \\
\hline Oceober & 138 & 4500 & 4638 & -0.51 & 450 & 3.99 & 0.00 & 828 & 0.00 & 0.00 \\
\hline November & -0.59 & 4500 & 4AA1 & -187 & 450 & 2.63 & 0.00 & 6.64 & -1.03 & 0.00 \\
\hline December & $-1.7 \pi$ & 45.00 & 4323 & -2800 & 4.50 & 1.70 & 0.00 & 5.65 & 581 & 0.00 \\
\hline
\end{tabular}

CLOUDY DAY HEAT BALANCE

\begin{tabular}{|c|c|c|c|c|c|c|c|c|c|c|}
\hline \multirow[t]{2}{*}{ Manth } & \multicolumn{3}{|c|}{ Ocapiod Penod W/m2 } & \multicolumn{3}{|c|}{ Unocapiod PeriodW/m2 } & \multicolumn{4}{|c|}{ Monfiv Rearrement KWh Fresh ÁrLoadKWh } \\
\hline & PV netgain & Intornd's & Toveral & PYratgin & Intomels & Overal & Heaing & Cooting & Hoaling & Coofing \\
\hline Januay & -237 & 45.00 & 4263 & -292 & 450 & 158 & 0.00 & 9.06 & -948 & 0.00 \\
\hline Fobruary & -209 & 45.00 & 4281 & -285 & 450 & 1.65 & 0.00 & 6.96 & -728 & 0.00 \\
\hline March & -1.03 & 45.00 & 4397 & -205 & 450 & 245 & 0.00 & 7.35 & 521 & 0.00 \\
\hline Appil & 0.46 & 45.00 & 45.46 & -0.82 & 450 & 3.68 & 0.00 & 6.58 & $-1,31$ & 0.00 \\
\hline May & 1.68 & 45.00 & 46.68 & -0.16 & 450 & 434 & 0.00 & 6.38 & 0.00 & 0.00 \\
\hline Juno & $2 A 2$ & 4500 & $47 A 2$ & 0.30 & 450 & 4.83 & 0.00 & 5.04 & 0.00 & 182 \\
\hline Juty & 275 & 4500 & 47.75 & 036 & 450 & 486 & 0.00 & 4.74 & 0.00 & 227 \\
\hline August & 2,47 & 45.00 & 47,47 & 023 & 4.50 & 4.73 & 0.00 & 5.03 & 0.00 & 2.13 \\
\hline Soprember & 157 & 4500 & 4657 & -0.12 & 450 & 438 & 0.00 & 5.06 & 0.00 & 0.33 \\
\hline locidber & 024) & 45.00 & 4524 & -0.61 & 4.50 & 3.89 & 0.00 & 6.13 & 0.00 & 0.00 \\
\hline Novembor & $-1 A 0$ & 1500 & 13.60 & -1900 & 450 & 2.60 & 0.00 & 7.65 & 4.73 & 0.00 \\
\hline locournbor & -246 & 45.00 & 4254 & -289 & 450 & 1.69 & 0.00 & 8.35 & -872 & 0.00 \\
\hline
\end{tabular}

OVERMII ERERGY REOURELEETS AND PRODUCTIOH

\begin{tabular}{|c|c|c|c|c|c|c|c|}
\hline \multirow[t]{2}{*}{ Month } & \multicolumn{2}{|c|}{ Cooting Consumpion } & \multicolumn{2}{|c|}{ Heating Consumption } & \multirow{2}{*}{$\begin{array}{l}\text { Fans } \\
\text { kMm }\end{array}$} & \multirow{2}{*}{$\begin{array}{l}\text { Lighting and } \\
\text { Pomor kWh }\end{array}$} & \multirow{2}{*}{$\begin{array}{l}\text { Tobal kWh } \\
\text { Consumad }\end{array}$} \\
\hline & $1 / 000$ & kHh Eloctic & $1 / 0, p$ & kth Eectic & & & \\
\hline January & 0.00 & 0.00 & 022 & 323 & 3.77 & 8.84 & 158 \\
\hline February & 0.00 & 0.00 & 022 & 294 & 340 & 798 & 14 \\
\hline March & 0.00 & 0.00 & 022 & 232 & 3.77 & 8.84 & 14. \\
\hline Apre & 0.29 & 446 & 022 & 0.65 & 3.55 & 8.55 & $1 \%$ \\
\hline $\mathrm{Mzy}$ & 028 & 4283 & 022 & 0.00 & 3.77 & 8.84 & 17. \\
\hline Juno & 029 & $6 A 7$ & 022 & 0.00 & 3.55 & 8.55 & 18.6 \\
\hline Juty & 0.29 & 729 & 0.22 & 0.00 & 3.77 & -8.84 & 198 \\
\hline August & 028 & 6.94 & 0.22 & 0.00 & 3.77 & 8.84 & 18.5 \\
\hline Septentber & 028 & 4.92 & 0.22 & 0.00 & 3.55 & 8.55 & 17.12 \\
\hline Octobser & 029 & 4.56 & 022 & 0.00 & 3.77 & 8.84 & 17. \\
\hline Norember & 0.00 & 0.00 & 022 & 195 & 3.55 & 8.55 & 14. \\
\hline Decomber & 0.00 & 0.00 & 0.22 & 323 & 3.77 & 8.84 & 1583 \\
\hline TOTAL/m12 & & 39.46 & & 1433 & 44.35 & 104.03 & 2021 \\
\hline
\end{tabular}


OPTION 4: $\quad$ PV ROOF MONITORS

\begin{tabular}{|c|c|c|c|c|c|c|c|c|c|c|}
\hline $\begin{array}{l}\text { Cay } \\
\text { Crmato }\end{array}$ & $\begin{array}{l}\text { San Francisos } \\
\text { Temperab oos }\end{array}$ & $\begin{array}{l}\infty \\
\text { ostal wam }\end{array}$ & $\begin{array}{l}\text { Longinde } \\
\text { Lafiteds } \\
\text { Alísode }\end{array}$ & $\begin{array}{r}37.62 ! \\
12238 \\
2.00\end{array}$ & & $\begin{array}{l}\text { Direct Radaio } \\
\text { Dithiso Rediai }\end{array}$ & $\begin{array}{l}\text { ion Factox } \\
\text { ion Factor }\end{array}$ & $\begin{array}{l}0.50 \\
1.10\end{array}$ & & \\
\hline \multicolumn{11}{|c|}{ WEATHERDATA } \\
\hline \multirow[t]{2}{*}{ Month } & Trpical Dary T & Temponitus & Radation $24 x$ & ravorages & Hours of Sans & $\sin$ & Equivent Day & & Averapo Hum & 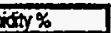 \\
\hline & $\operatorname{Max} \propto$ & Hnoc & Droct H/hn2 & Dithuse W/ma & Tot hars & Haxper day & Sumy & Coudy & Day & Night \\
\hline January & 1300 & 5.40 & 8500 & 40.00 & 154.00 & 10.00 & 15.40 & 15.60 & 75 & 85 \\
\hline Fabruary & 14.30 & 6.30 & 130.00 & 55.00 & 191.00 & 11.56 & 16.52 & 11.49 & 71 & 85 \\
\hline Warch & 16,40 & 720 & 180.00 & 75.00 & 20200 & $12 / 3$ & 2108 & 992 & 66 & 83 \\
\hline Apri & 1790 & 8.40 & 250.00 & 100.00 & 297.00 & 1394 & 2130 & 8.70 & 66 & 85 \\
\hline Hity & 19.40 & 9.80 & 290.00 & 120.00 & 328.00 & 1490 & 2201 & 899 & 65 & 85 \\
\hline fino & 2120 & 11.30 & 305.00 & 125.00 & 34100 & 1536 & 2220 & 780 & 65 & 87 \\
\hline fuly & 2200 & 11.90 & 290.00 & 120.00 & 205.00 & 1420 & 20.7 & 1023 & $\infty$ & 90 \\
\hline August & 2210 & 1210 & 250.00 & 100.00 & 275.00 & 13.65 & 20.15 & 10.85 & $\infty$ & 90 \\
\hline Soptonbor & 2320 & 1220 & 190.00 & 7500 & 25000 & 1273 & 21.60 & 8.40 & 66 & 88 \\
\hline Octobox & 2110 & 1030 & 130.00 & 5500 & 24200 & 1131 & $21 \approx 0$ & 9.61 & 65 & 86 \\
\hline Norember & 1780 & 7.70 & 8500 & 40.00 & 188000 & 10.00 & 1980 & 1020 & 68 & 84 \\
\hline Docartiber & 14.00 & 6.10 & 7000 & 3500 & 179.00 & 1031 & 1736 & 13.64 & 74 & 86 \\
\hline BURDANGD & ATA & & & & PVPAKEIDA & ATA & & & HUDDTYभI & TIER \\
\hline $\begin{array}{l}\text { Area of PVP } \\
\text { Area of Nort } \\
\text { Heat Gain to } \\
\text { Heat Gain fro }\end{array}$ & $\begin{array}{l}\text { and porm2 of } \\
\text { h fight per m2 of } \\
\text { on lights Whe } \\
\text { om Equipment }\end{array}$ & $\begin{array}{l}\text { Illoor } \\
\text { of boor } \\
12 \\
\text { IW/m2 }\end{array}$ & $\begin{array}{r}1.00 \\
0.15 \\
20.00 \\
5.00\end{array}$ & & $\begin{array}{l}\text { Conversion En } \\
\text { Diroct Transmi } \\
\text { Thermal Trans } \\
\text { PV slope ( }\end{array}$ & $\begin{array}{l}\text { incioncy } \\
\text { nitanco } \\
\text { smitanco With }\end{array}$ & $n$ & $\begin{array}{r}10.00 \\
0.00 \\
020 \\
15.00 \\
\end{array}$ & $\begin{array}{l}\text { FFH } \\
\text { Entraloy }\end{array}$ & $\begin{array}{r}35 \\
3641\end{array}$ \\
\hline Hot Gainto & on Peoplo Whr & & 2000 & & INERRAL TE & EIPERATURE & & & HULPDTS & UTLER \\
\hline $\begin{array}{l}\text { Oocopancys } \\
\text { Ocomancy F }\end{array}$ & $\begin{array}{l}\text { Start Tme } \\
\text { Frish Trme }\end{array}$ & & $\begin{array}{r}9.00 \\
19.00\end{array}$ & & $\begin{array}{l}\text { Ocapiad Pax } \\
\text { Unocapiod P }\end{array}$ & $\begin{array}{l}\text { rod Set Point } \\
\text { Period Otsol }\end{array}$ & $\begin{array}{l}\infty \\
\infty\end{array}$ & $\begin{array}{r}21.00 \\
5.00\end{array}$ & Entraby & \begin{tabular}{r|}
60 \\
47.425 \\
\end{tabular} \\
\hline CALCUAT & TONS OFMEY & MEXTERTLL & CONDIIONS & & & & & & & \\
\hline Manth & Tomperatrao & $\alpha$ & Sumy Day Ra & adabonW/ma/C & Cloudy Day R: & Zadion W/ma & Equivient Day & & ExtEntraloy & $\mathrm{k} / \mathrm{kg}$ \\
\hline & Oocamiod & Thoocapiod & Oocapied & Uhocasiod & loospiod I & Uhocosiod & Sumy 10 & Cowy & Docupiod & throcapiod \\
\hline Janay & 11.10 & 784 & 207.60 & 0.00 & 9600 & 0.00 & 15.10 & 1560 & 2801 & 2252 \\
\hline Fobruery & 1260 & 9.00 & 26051 & 29.06 & 114.17 & 1274 & 1652 & 11.48 & 30.55 & 25.19 \\
\hline Warch & 14.90 & 10.16 & 34275 & 5946 & 1488 & 2513 & 21.08 & 9.22 & 3271 & 2753 \\
\hline Apri & 1553 & $11 / 5$ & 404AB & 11394 & 172.12 & 48.48 & 2130 & 8.70 & 36,08 & 31.15 \\
\hline Wity & 17.00 & 1280 & 46.11 & 15621 & 18026 & 67.67 & 2201 & 899 & 39.69 & 34.84 \\
\hline fino & 18.73 & $14 A 8$ & 453.11) & 173.49 & 185.31 & 74.78 & 220 & 7.80 & 4367 & $3 . \infty$ \\
\hline Juty & 19.63 & 1521 & 468.06 & 14053 & $m \pi$ & 60.88 & 20.77 & 1023 & 47.52 & 4264 \\
\hline Aurgest & 19.60 & 1531 & 41326 & 107.67 & 175.85 & 4582 & 20.15 & 10.85 & 47.55 & 4294 \\
\hline September & 20.45 & 15.74 & 334.60 & 6528 & 14138 & 2758 & 21.60 & 840 & 4859 & 4354 \\
\hline foctober & 18.63 & 1337 & 26623 & 2498 & 116.57 & 10.25 & 2139 & 9.61 & 48,2 & 37.72 \\
\hline Noverber & 1528 & 10.05 & 207.50 & 0.00 & 9600 & 0.00 & 1080 & 1020 & 36.10 & 29.66 \\
\hline Docomber & 1203 & 8.64 & 171.08 & 380 & B1A거 & 1.81 & 17.36 & 1364 & 29.94 & 2453 \\
\hline SURETDAY & Y HEAT BNAN & UKCE & & & & & & & & \\
\hline Month & Decapied Peix & iod W/m2 & & Unocosiod $P_{0}$ & aciodW/m2 & & Monthy Roqi & ramentiky & Fiosh ir Loa & $d x W h$ \\
\hline & PVnotgain & Thotrals & Ooral & PVnetgin & Thromals & Ovall & Haring & $\operatorname{cosin} \theta$ & Hoaing & $\operatorname{cosing}$ \\
\hline Jaxway & 4.60 & 25.00 & 2031 & -530 & 450 & -0.80 & $-0,17$ & 3.13 & -248 & 0.00 \\
\hline Fabruary & 327 & 25.00 & 21.73 & 431 & 450) & 0.19 & 000 & 3.63 & -1.88 & 0.00 \\
\hline March & -1.51 & 25.00 & 2339 & 330 & 450 & 120 & 0.00 & 529 & -1.50 & 0.00 \\
\hline Apri & -0.16 & 2500 & 24.84 & -200 & 450 & 250 & 0.00 & 6.04 & -0.14 & 0.00 \\
\hline May & 1.15 & 25,00 & 26.15 & -180 & 450 & 270 & 0.00 & 6.59 & 0.00 & 0.00 \\
\hline Hino & 233 & 25.00 & 2733 & -4.15 & 450 & 3.35 & 0.00 & 7.11 & 0.00 & 0.00 \\
\hline fuly & 3.04 & 25.00 & 2804 & -0.96 & 450 & 354 & 0.00 & 6.85 & 0.00 & 0.08 \\
\hline August & 258 & 2500 & 2756 & -1.17 & 450 & 3.33 & 0.00 & 6.99 & 0.00 & 0.05 \\
\hline Septenber & 245 & 2500 & 27.45 & -125 & 450 & 325 & 0.00 & 691 & 0.00 & 0.48 \\
\hline Dotaber & 0.60 & 2500 & 2569 & -1.18 & 450 & 332 & 0.00 & 6.49 & 0.00 & 0.00 \\
\hline Norarnber & -1.98 & 25.00 & 2302 & 328 & 450 & 122 & 0.00 & 400 & -0.12 & 0.00 \\
\hline Decorniber & 440 & 25.00 & 20.60 & 4.75 & 450 & .025 & -0.06 & 358 & .216 & 0.00 \\
\hline CLOSOYDA & TYIEAT $8 A L A$ & ANCE & & & & & & & & \\
\hline Month & Docalod Pen & nodW/m2 & & Unooproied Pe & eriodW/m2 & & Monhty Regir & rerrentkWh & IFrosh Aíloas & dkHh \\
\hline & PVnetgain & Inwards & Overal & PVnotoin & Intmols & Overal & Hoaing Io & Cooing & Hoaing & $\cos i n$ \\
\hline fancary & 55 & 4500 & 3937 & 530 & 450 & -0.80 & -0.18 & 6.14 & -252 & 0.00 \\
\hline Fabruary & 450 & 40.00 & 3550 & 4.44 & 450 & 0.06 & 0.00 & 4.08 & -129 & 0.00 \\
\hline March & -327 & 3500 & 31.73 & 359 & 450 & 0.97 & 0.00 & 327 & -0.71 & 0.00 \\
\hline Apri & -211 & 30.00 & 2789 & -255 & 4.50 & 1.95 & 0.00 & 2.66 & -0.06 & 0.00 \\
\hline Way & -0.98 & 2500 & 24.02 & -146 & 450 & 3.04 & 0.00 & 254 & 0.00 & 0.00 \\
\hline Jino & 0.16 & 2500 & 25.16 & -0.36 & 450 & 4.14 & 0.00 & 211 & 0.00 & 0.00 \\
\hline Juty & 0.81 & 2500 & 2581 & 0.00 & 450 & 4.50 & 0.00 & 328 & 0.00 & 0.04 \\
\hline August & 0.57 & 2500 & 25.57 & -0.06 & 450 & 44 & 0.00 & 3.45 & 0.00 & 0.03 \\
\hline Seprember & 0.83 & 30.00 & 30.83 & 0.06 & 4.50 & 456 & 0.00 & 3.13 & 0.00 & 0.19 \\
\hline lotaber & -0.56 & 35.00 & $34 M$ & -129 & 450 & 321 & 0.00 & 3.74 & 0.00 & 0.00 \\
\hline Noventer & -291 & 40.00 & 37.09 & 328 & 450 & 122 & 0.00 & 395 & -0.06 & 0.00 \\
\hline December & -5.15 & 45.00 & 39.85 & -4.77 & 450 & .027 & -0.05 & 5.4 & -1.69 & 0.00 \\
\hline OYERAIIE & RERGYREQL & RREREKTS A & WDPRODUCT & ITON & & & & & & \\
\hline Manth & Coding Cons & ympion & Heaing Cons & unotion & Fans & Lighing and & Tobl kWh & & & \\
\hline & 11009 & Khotectic & $1 / 000$ & kum Eoctic & km & Power kWh & Corsemed & & & \\
\hline Januay & 0.00 & 0.00 & 022 & 1.19 & 2.08 & 5.76 & 9.03 & & & \\
\hline Fobruary & 0.00 & 0.00 & 022 & 0.70 & 1.88 & 4.10 & 6.68 & & & \\
\hline Narch & 0.00 & 0.00 & 022 & 0.49 & 208 & 3.60 & 620 & & & \\
\hline Apri & 029 & 2.49 & 022 & 0.04 & 2.01 & 299 & 7.53 & & & \\
\hline May & 029 & 261 & 022 & 0.00 & 208 & 2.64 & 7.33 & & & \\
\hline Jino & 029 & 2.72 & 022 & 0.00 & 201 & 255 & 728 & & - & \\
\hline Juty & 029 & 293 & 022 & 0.00 & 208 & 264 & 7.65 & & & \\
\hline August & 029 & 286 & 022 & 0.00 & 200 & 264 & 758 & & & \\
\hline Sopermber & 029 & 3.06 & 022 & 0.00 & 201 & 2.97 & 8.04 & & & \\
\hline October & 029 & 298 & 022 & 0.00 & 2.08 & 3.60 & 8.60 & & & \\
\hline Noverrber & 029 & 253 & 022 & 0.04 & 2.01 & 4.08 & 8.66 & & & \\
\hline Decerrbor & 0.00 & 0.00 & 022 & 0.88 & 2.08 & 5.35 & 8.33 & & & \\
\hline TOTAL/m2 & & 2212 & & 334 & 24.51 & 4293 & 9290 & & & \\
\hline
\end{tabular}


OPTION 4: PYROOF HONITORS

\begin{tabular}{|c|c|c|c|c|c|c|c|c|c|c|}
\hline \multirow{4}{*}{$\begin{array}{l}\text { CAY } \\
\text { Crinats } \\
\text { WEATHERD } \\
\text { Month }\end{array}$} & \multicolumn{2}{|c|}{$\begin{array}{l}\text { Now Yark } \\
\text { Tempante ocstal oild }\end{array}$} & $\begin{array}{l}\text { Longerde } \\
\text { lafude } \\
\text { Alituds }\end{array}$ & \multicolumn{2}{|c|}{$\begin{array}{r}40.70 \mathrm{~N} \\
74.02 \mathrm{~W} \\
3.00 \mathrm{~m}\end{array}$} & \multicolumn{2}{|c|}{$\begin{array}{l}\text { Drect Radaion Factor } \\
\text { Dirtuso Radafon Factx }\end{array}$} & $\begin{array}{l}0.50 \\
1.10\end{array}$ & & \\
\hline & & & & & & & & & & \\
\hline & \multicolumn{4}{|c|}{ 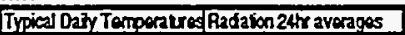 } & \multicolumn{2}{|c|}{ Hours ol Sunstine } & \multicolumn{2}{|c|}{ Egrialent Dars } & \multicolumn{2}{|c|}{ Avarag Humitiy $\%$} \\
\hline & MaxoC & Minoc & Dred Hinn2 & Dinuso Ying & Totahors & Maxporday & Surmy & County & Day & Noth \\
\hline Janary & 430 & -280 & 65.00 & 40.00 & 151.00 & 10.00 & 15.10 & 1590 & 61 & 6 \\
\hline Fobruery & 4.10 & -240 & 130,00 & 55.00 & 164.00 & 1028 & 15.95 & 1204 & 58 & 67 \\
\hline March & 890 & 0.60 & 190,00 & 75.00 & 209.00 & 1183 & 17.67 & 1333 & 56 & 67) \\
\hline Apri & 1490 & 6.10 & 25000 & 100.00 & 214.00 & 1209 & 17.70 & 1230 & 55 & 68 \\
\hline Mey & 2090 & 1180 & 29000 & 120.00 & 250.00 & 1344 & 1860 & 1240 & 56 & 60 \\
\hline Jun & 25.60 & 1690 & 205.00 & 125.00 & 301.00 & 16.18 & 18.60 & 11,40 & 58 & 72 \\
\hline Juty & 2930 & 19.90 & 29000 & 120.00 & 307.00 & 15.00 & 20.46 & 1054 & 57) & 74 \\
\hline August & $27 A 0$ & 19.30 & 250,00 & 100.00 & 275.00 & 1386 & 19.84 & 11.16 & 60 & 76 \\
\hline Seplomber & 2380 & 1580 & 190,00 & 75.00 & 237.00 & 1254 & 1890 & 11.10 & 60 & 76 \\
\hline Octobes & 1840 & 1030 & 130.00 & 55.00 & 218.00 & 1134 & 19.22 & 11.78 & 59 & 74 \\
\hline Nowember & 11.90 & 440 & 8500 & 40.00 & 17200 & 10.06 & 17.10 & 1290 & 60 & 71 \\
\hline Docentex & 5.70 & -120 & 70.00 & 3500 & 158.00 & 10.00 & 1580 & 1520 & 60 & 67 \\
\hline BULLANG & ATA & & & & PVPAKEL D & ATA & & & HUDOTYW & WTER \\
\hline $\begin{array}{l}\text { Aros of PV } \\
\text { Area of Nor } \\
\text { Hoat Gain } \\
\text { Heat Gain }\end{array}$ & $\begin{array}{l}\text { aral pex m2 of } \\
\text { aghtper m2 of } \\
\text { xn Lights Whr } \\
\text { xn Equipment }\end{array}$ & $\begin{array}{l}f \text { foor } \\
\text { of froor } \\
2 \\
\text { Whn2 }\end{array}$ & $\begin{array}{r}1.00 \\
0.15 \\
20.00 \\
5.00\end{array}$ & & $\begin{array}{l}\text { Corverson E } \\
\text { Diroct Transm } \\
\text { Thormal Tran } \\
\text { PV } \operatorname{siope}\left({ }^{\circ}\right)\end{array}$ & $\begin{array}{l}\text { Eniciency } \\
\text { nitance } \\
\text { smitanco Wh }\end{array}$ & $m 20 c^{\%}$ & $\begin{array}{r}10.00 \\
0.00 \\
0.20 \\
15.00\end{array}$ & $\begin{array}{l}\text { EFH } \\
\text { Entrapy }\end{array}$ & $\begin{array}{r}35 \\
36.41\end{array}$ \\
\hline Heat Gain & m Peoplo W/m & & 20.00 & & HTERMLI T & EXPERATLR & & & HLATITYST & UNIER \\
\hline $\begin{array}{l}\text { Ocapany } \\
\text { Ocapany }\end{array}$ & $\begin{array}{l}\text { Thert Trme } \\
\text { rist Tme }\end{array}$ & & $\begin{array}{r}8.00 \\
19.00\end{array}$ & . & $\begin{array}{l}\text { Cocupiod Pon } \\
\text { Unocoupiod P }\end{array}$ & $\begin{array}{l}\text { nod Sol Point } \\
\text { Period Otiset }\end{array}$ & $\begin{array}{l}\infty \\
\infty\end{array}$ & $\begin{array}{r}21.00 \\
5.00\end{array}$ & $\begin{array}{l}\text { \%AF } \\
\text { Entaby }\end{array}$ & $\begin{array}{r}60 \\
47.425 \\
\end{array}$ \\
\hline CALCUA & OHS OF UEA & WEXTEPULL & $\perp$ COHDITOKS & & & & & & & \\
\hline Manth & Temporatre & & Sumy DayR & actation W/ma & Cloudy Day $\mathrm{B}$ & adation What & Egrialent Da & & Ex Entraloyl & $\mathrm{k} / \mathrm{kg}$ \\
\hline & Ocapied & Uhoosupiod & Oosipiod & Uhoconied & oonoiod & Urrocenied & $5 \mathrm{sm}$ & coovoly & Oocopied & Lrocaniad \\
\hline Jancery & 253 & -0.52 & 207.60 & 0.00 & 96.00 & 0.00 & 15.10 & 1590 & 895 & 483 \\
\hline Fabrary & 248 & -0.31 & 293.12 & 5.7 & 128.46 & 253 & 1596 & 1204 & 8.56 & 490 \\
\hline March & 683 & 327 & 360.16 & 47.03 & 152.18 & 1987 & 17.67 & 1333 & 15.67| & 1088 \\
\hline Apri & 1270 & 893 & $466 \wedge 9$ & 69.65 & 198.50 & 29.64 & 17.70 & 1230 & 26.71 & 2180 \\
\hline Ming & 18.63 & 14.73 & 494.61 & 121.56 & 21427 & 5266 & 18.60 & 1240 & 39.88 & 3.06 \\
\hline June & 2343 & 19.70 & 43000 & 189.94 & 18538 & 8187 & 1860 & 1140 & 5274 & 49.03 \\
\hline Juty & 2620 & 2260 & 443.06 & 158,39 & 191.24 & 6862 & 20.46 & 10.54 & 59.60 & 5233 \\
\hline August & 2538 & 2180 & 406.90 & 11221 & 173.15 & 47.75 & 1984 & 11.16 & 5906 & 57.18 \\
\hline soptentior & 2180 & 1837 & 339.72 & 61.53 & 143.54 & 26.04 & 1890 & 11.10 & 49.48 & 4690 \\
\hline Octaber & 1638 & 1290 & 265.55 & 2546 & 116.38 & 11.16 & 1922 & 11.78 & 3584 & 3204 \\
\hline Novenber & 10.03 & 681 & 20639 & 086 & 854 & 040 & 17.10 & 1200 & 2245 & 1801 \\
\hline Docember & 3.88 & 1.02 & 17640 & 0.00 & 84.00 & 0.00 & 15.80 & 1520 & 1126 & 7.09 \\
\hline SLAYYOA & THEAT BALNS & KCE & & & & & & & & \\
\hline Manth & Ocapiod Peo & xiod W/m2 & & Unocapiod P & PeriodW/m2 & & Monsty Reau & trementkWh & Ifrestifíloa & $\mathrm{d} \mathrm{kWh}$ \\
\hline & PVnotgin & Inmerals & Oomel & Pynotoin & Intome's & Oreal & Heasing & Cooing & Heasing & Coofing \\
\hline Jaxuary & -1026 & 25.00 & 0 14.74 & -10.74 & 4.50 & -624 & $-1,32$ & 220 & -7.96 & 0.00 \\
\hline Fobreary & -9.58 & 2500 & 15.42 & -10.55 & 450 & -6.05 & -135 & 246 & -8.54 & 0.00 \\
\hline March & -6.19 & 25.00 & 18.81 & -7.88 & 450 & 338 & -084 & 332 & .7 .04 & 0.00 \\
\hline Apri & -148 & 2500 & 2052 & -401 & 450 & 0.48 & 0.00 & 428 & 330 & 0.00 \\
\hline $\mathrm{May}$ & 2.61 & 2500 & 27.61 & -306 & 4.50 & $1 \mathrm{~A}$ & 0.00 & 5.51 & 0.00 & 0.00 \\
\hline Jino & 5.19 & 2500 & 30.18 & $-1 A 2$ & 450 & 3.08 & 0.00 & $6 / 2$ & 0.00 & 190 \\
\hline Juty & 7.10 & 25.00 & 3210 & -0.88 & 450 & 3.62 & 0.00 & 7.61 & 0.00 & 4.78 \\
\hline Auguse & 626 & 2500 & 3126 & -0.64 & 450 & 3.86 & 0.00 & 728 & 0.00 & 4.43 \\
\hline Soptrmber & 337 & 25.00 & 2837 & -1.19 & 4.50 & 3.31 & 0.00 & 624 & 0.00 & 0.75 \\
\hline Cotobor & -0.78 & 25.00 & 2422 & -180 & 450 & 270 & 0.00 & 5.39 & $-22 i$ & 0.00 \\
\hline Norember & 540 & 2500 & 19.60 & -5.97 & 450 & $-1 A 7$ & -0.35 & 335 & -4.58 & 0.00 \\
\hline December & -9.58 & 25.00 & 15,42 & -9.74 & 450 & .524 & -1.16 & 244 & -7.63 & 0.00 \\
\hline CLOWOYD & TYEATBAL & ANCE & & & & & & & & \\
\hline Manth & Oocpiod Pen & riod W/m2 & & UnocapiodP & Tim2 & & $4 \operatorname{Req}$ & thWh & Airlos & \\
\hline & PVnotgin & Intorrals & Oarel & PVnolgin & Intana's & Ouaral & Heating & $\operatorname{cosing}$ & Heaing & Cooling \\
\hline Janery & -1120 & 4500 & 3380 & -10.74 & 450 & 624 & -1.39 & 5.37 & 838 & 0.00 \\
\hline Fabrey & -10.96 & 40.00 & $290 \mathrm{~A}$ & -10.58 & 450 & -608 & -1.02 & 350 & 6.44 & 0.00 \\
\hline March & .724 & 35.00 & 27.06 & 8.11 & 450 & -361 & -0.67 & 3.61 & 531 & 0.00 \\
\hline Apri & 3.73 & 30.00 & 2627 & -435 & 450 & 0.15 & 0.00 & 326 & -229 & 0.00 \\
\hline May & 0.26 & 2500 & 2526 & -0.39 & 4.50 & 4.11 & 0.00 & 3.85 & 0.00 & 0.00 \\
\hline Juno & 3.13 & 25.00 & 28.13 & -0.48 & 4.50 & 4.02 & 0.00 & 3.85 & 0.00 & 1.16 \\
\hline Juty & 4.99 & 2500 & 29.99 & -0.01 & 450 & 449 & 0.00 & 382 & 0.00 & 246 \\
\hline August & 430 & 25.00 & 29.30 & -0.09 & 4.50 & 4A1 & 0.00 & 3.86 & 0.00 & 249 \\
\hline Soplanber & 1.73 & 30.00 & 31.73 & -1.16 & 4.50 & 3.34 & 0.00 & 4.04 & 0.00 & 0,4 \\
\hline octobor & -200 & 35.00 & 3297 & -1.92 & 450 & 258 & 0.00 & 431 & -0.13 & 0.00 \\
\hline Novermber & 633 & 40.00 & 33.67 & 5.97 & 450 & -1.47 & -027 & 434 & 246 & 0.00 \\
\hline Docember & -10.36 & 45.00 & 34.64 & -9.74 & 4.50 & .524 & -1.11 & 527 & .734 & 0.00 \\
\hline
\end{tabular}

OVERALL ERERGY REOUIREUENTS ANO PRODUCTON

\begin{tabular}{|c|c|c|c|c|c|c|c|}
\hline \multirow[t]{2}{*}{ Mon'n } & \multicolumn{2}{|c|}{ Coding Consumpion } & \multicolumn{2}{|c|}{ Hoaling Consurnotion } & \multirow{2}{*}{$\begin{array}{l}\text { Fans } \\
\text { kwin }\end{array}$} & \multirow{2}{*}{$\begin{array}{l}\text { Lighting and } \\
\text { Power KWh }\end{array}$} & \multirow{2}{*}{$\begin{array}{l}\text { Tosal kWh } \\
\text { Consernod }\end{array}$} \\
\hline & 1000 & KWh Eoctic & $1 \log \rho$ & LWhEsctic & & & \\
\hline Januay & 0.00 & 0.00 & 022 & 423 & 230 & 582 & 1235 \\
\hline Fobruay & 0.00 & 0.00 & 022 & 3.86 & 208 & 4.19 & 10. \\
\hline March & 0.00 & 0.00 & 022 & 3.08 & 230 & 3.97 & 9 \\
\hline Apri & 0.00 & 0.00 & 022 & 124 & 223 & 3.17 & 6.6 \\
\hline May & 0.29 & 267 & 022 & 0.00 & 230 & 264 & 7.61 \\
\hline Jino & 0.20 & 381 & 022 & 0.00 & 223 & 255 & 85 \\
\hline Jaty & 029 & 534 & 022 & 0.00 & 230 & 2.64 & 102 \\
\hline August & 0.20 & 5.18 & 022 & 0.00 & 2.30 & 2.64 & 10 \\
\hline Septamber & 0.29 & 327 & 022 & 0.00 & 223 & 3.11 & 8. \\
\hline Octaber & 029 & $2 \pi 7$ & 022 & 0.08 & 230 & 3.81 & 89 \\
\hline Novernber & 0.00 & 0.00 & 022 & 1.92 & 223 & 449 & 8.63 \\
\hline Docombar & 0.00 & 0.00 & 022 & 3.83 & 2.30 & 5.68 & 1181 \\
\hline TOTALm2 & & 23.05 & & 18.25 & 27.07 & 44.67 & 113.04 \\
\hline
\end{tabular}


OPTION 4:

PV ROOF HONITORS

STEDATA

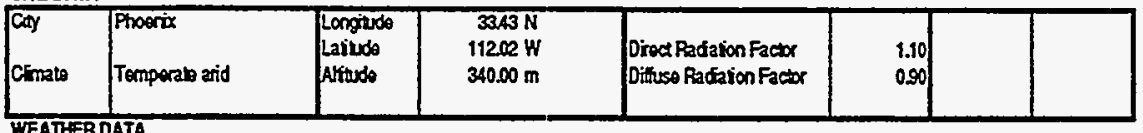

WEATHERDATA

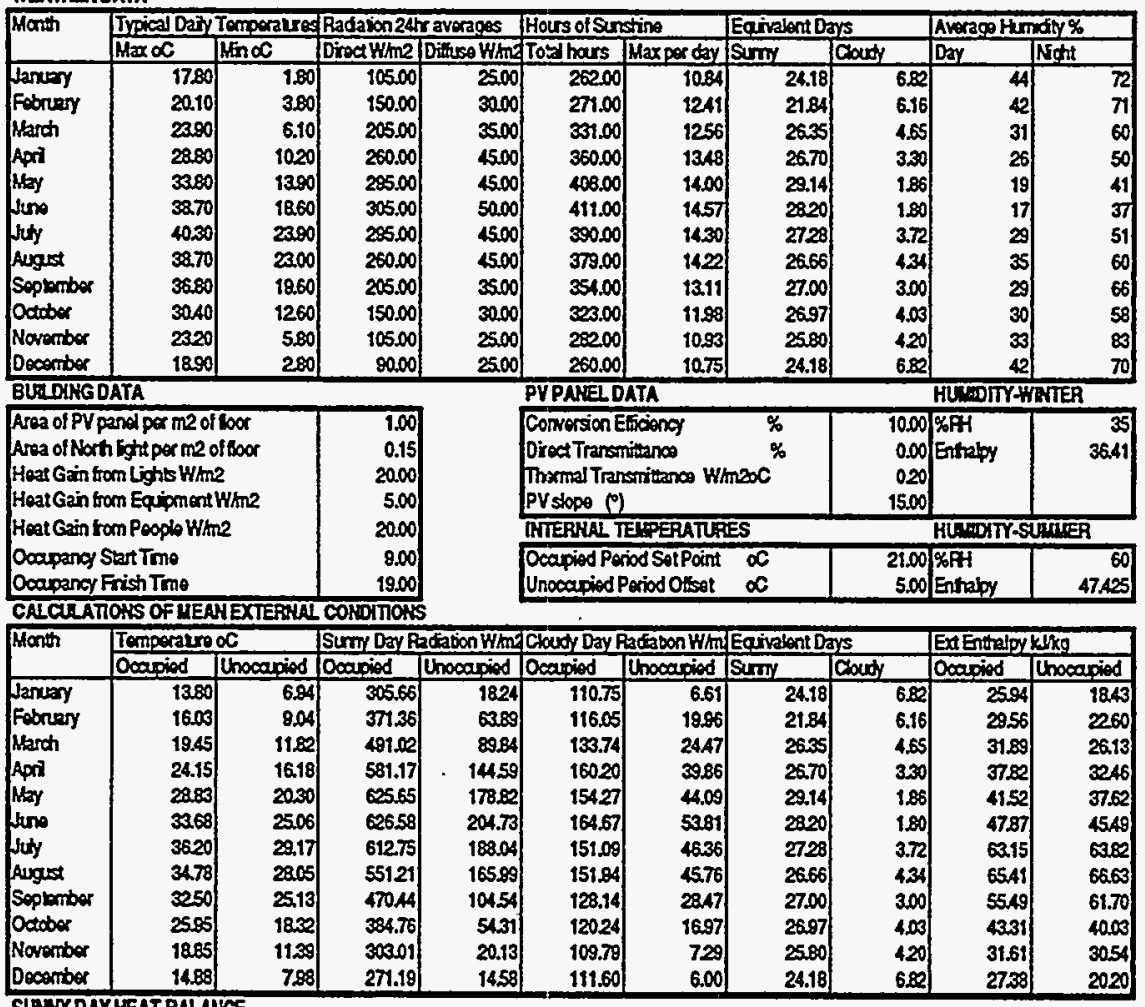

SLWWY DAYKEAT BALAYCE

\begin{tabular}{|c|c|c|c|c|c|c|c|c|c|c|}
\hline \multirow{2}{*}{ Month } & \multicolumn{3}{|c|}{ Ooconied Period W/m2 } & \multicolumn{3}{|c|}{ Unocoupiod Poriod Whm2 } & \multicolumn{4}{|c|}{ Monthy RegiromontkWh Fesh Air LoadkWh } \\
\hline & Pynatgin & Intmis & Overa & PVmatgin & Entrols & Oreral & Heasisg & Cooling & Healing & Cooing \\
\hline Jaxbery & -2.11 & 25.00 & 2289 & 5.73 & 4.50 & -123 & -0.12 & $5 \mathbf{5 3}$ & 486 & 0.0 \\
\hline Fobriay & -0.11 & 25.00 & 2489 & 3909 & 450 & 0.51 & 0.00 & 559 & -288 & 0.00 \\
\hline Merch & 3.12 & 2500 & 28.12 & -1.86 & 450 & 254 & 0.00 & 835 & -220 & 0.00 \\
\hline Apre & 6.83 & 2500 & 31.93 & -1.82 & 450 & 258 & 0.00 & 9.49 & 0.00 & 0.0 \\
\hline May & 1034 & 2500 & 3534 & 1.04 & 450 & 5.54 & 0.00 & 1256 & 0.00 & 0.00 \\
\hline Juno & 1350 & 2500 & 3850 & 1.11 & 450 & 5.67 & 0.00 & 13.07] & 0.00 & 02 \\
\hline fuldy & 15.03 & 25.00 & 40.03 & 3.64 & 4.50 & 8.14 & 0.00 & 14.03 & 0.00 & 82 \\
\hline August & 1358 & 25.00 & 3858 & 272 & 450 & 722 & 0.00 & 1288 & 0.00 & 82 \\
\hline Sepraber & 11.43 & 2500 & 3643 & 0.31 & 450 & 481 & 0.00 & 11.65 & 0.00 & 4.18 \\
\hline Octobor & 6.45 & 2500 & 3145 & -128 & 4.50 & 322 & 0.00 & 8.70 & 0.00 & 0.00 \\
\hline November & 1.15 & 2500 & 26.15 & -263 & 450 & 1.67 & 0.00 & 735 & -238 & 0.00 \\
\hline Oecomber & -1.70 & 25.00 & 2330 & 5.09 & 450 & -0.59 & -020 & 5.63 & -4.19 & 0.00 \\
\hline
\end{tabular}

CLOTOY DAY HEAT BALAHCE

\begin{tabular}{|c|c|c|c|c|c|c|c|c|c|c|}
\hline \multirow[t]{2}{*}{ Month } & \multicolumn{3}{|c|}{ Oocoiod Period Whn } & \multicolumn{3}{|c|}{ Unocoupied Period W/ln? } & \multicolumn{4}{|c|}{ Mondily Regirament KWh Fresh Ai Load kWh } \\
\hline & PV not gain & Intenals & Oueral & PVmotgin & Intoma's & Oard & Heaing & $\operatorname{cocim}$ & Foaing & Cooling \\
\hline Jancay & -375 & 4500 & 4125 & 583 & 450 & -1.33 & -0.13 & 281 & -1.37 & 0.00 \\
\hline Fobruery & -2.26 & 40.00 & 37.74 & 436 & 450 & 0.14 & 0.00 & 234 & -0.81 & 0.00 \\
\hline March & 0.12 & 35,00 & 312 & -251 & 450 & 198 & 0.00 & 1.76 & -0.40 & 0.00 \\
\hline Apri & 3.39 & 30.00 & 3330 & -1.17 & 450 & 335 & 0.00 & 126 & 0.00 & 0.00 \\
\hline Why & 6.38 & 2500 & 3138 & -0.09 & 450 & 4A1 & 0.00 & 0.70 & 0.00 & 0.00 \\
\hline Jino & 9.62 & 25.00 & 34.62 & 147 & 450 & 5.97 & 0.00 & 0.7 & 0.00 & 0.02 \\
\hline Jithy & 11.15 & 2500 & 36.15 & 245 & 450 & 6.95 & 0.00 & 1.71 & 0.00 & 1.12 \\
\hline Augs: & 1023 & 2500 & 3523 & 1.74 & 450) & 621 & 0.00 & 191 & 0.00 & 150 \\
\hline Soplamber & 855 & 3000 & 3855 & -0.33 & 450 & 4.17 & 0.00 & 133 & 0.00 & 0.46 \\
\hline Oataber & 423 & 35.00 & 3923 & -1.60 & 450 & 200 & 0.00 & 1.74 & 0.00 & 0.00 \\
\hline Norentbor & -0.48 & 40.00 & 39.52 & -293 & 450 & 157 & 0.00 & 1.75 & -0.39 & 0.00 \\
\hline Decentiber & 3.04 & 45.00 & 41.26 & .5 .17 & 450. & -0.67 & -0.06 & 288 & -1.18 & 0.00 \\
\hline
\end{tabular}

OVERALI ERERGY REQURERERTS ALD PRODUCTON

\begin{tabular}{|c|c|c|c|c|c|c|c|}
\hline \multirow[t]{2}{*}{ Month } & \multicolumn{2}{|c|}{ Cooing Consimpion } & \multicolumn{2}{|c|}{ Heating Consumosion } & \multirow{2}{*}{$\begin{array}{l}\text { Fans } \\
\text { iknh }\end{array}$} & \multirow{2}{*}{$\begin{array}{l}\text { Lighting and } \\
\text { Powor kith }\end{array}$} & \multirow{2}{*}{$\begin{array}{l}\text { Total XHh } \\
\text { Consermod }\end{array}$} \\
\hline & $1 / 1000$ & Wwh Bectic & 11000 & Khn Eoctic & & & \\
\hline famery & 0.00 & 0.00 & 0.22 & 1.51 & 293 & 400 & 8.44 \\
\hline Fobruary & 029 & 227 & 022 & 0.82 & 2.65 & 3.3 & 9.04 \\
\hline March & 020 & 200 & 022 & 0.60 & 298 & 3.10 & 8.52 \\
\hline Aprad & 029 & 3.07 & 022 & 0.00 & 284 & 272 & 8.62 \\
\hline May & 0.29 & 3.79 & 022 & 0.00 & 293 & 264 & 9.36 \\
\hline Juno & 0.29 & 403 & 022 & 0.00 & 284 & 255 & 9.42 \\
\hline July & 029 & 7.17 & 022 & 0.00 & 293 & 264 & 1274 \\
\hline August & 022 & 731 & 022 & 0.00 & 293 & 26 & 1288 \\
\hline Seprenber & 029 & 5.04 & 022 & 0.00 & 284 & 270 & 1057 \\
\hline Oatober & 0.29 & 327 & 0.22 & 0.00 & 283 & 3.04 & 924 \\
\hline Norember & 029 & 2.60 & 022 & 0.61 & 284 & 3.18 & 923 \\
\hline Decamber & 0.00 & 0.00 & 022 & 1.25 & 2.93 & 4.00 & 8.18 \\
\hline TOTALm2 & & 41,43 & & 4.79 & 34.53 & 3648 & 11724 \\
\hline
\end{tabular}


OPTION 4: PV ROOF HONITORS

STIEDATA

\begin{tabular}{|c|c|c|c|c|c|}
\hline $\begin{array}{l}\text { City } \\
\text { cimato }\end{array}$ & $\begin{array}{l}\text { Hami } \\
\text { Stb-ropial }\end{array}$ & $\begin{array}{l}\text { Longudes } \\
\text { Laitudo } \\
\text { Afrudo }\end{array}$ & $\begin{array}{l}25.80 \mathrm{~N} \\
8027 \mathrm{~W} \\
200 \mathrm{~m}\end{array}$ & $\begin{array}{l}\text { Dirod Padaion Factor } \\
\text { Dituse Radition Factor }\end{array}$ & $\begin{array}{l}0.50 \\
1.10\end{array}$ \\
\hline
\end{tabular}

WEATHERDATA

\begin{tabular}{|c|c|c|c|c|c|c|c|c|c|c|}
\hline \multirow[t]{2}{*}{ Menth } & \multicolumn{4}{|c|}{ Trpical Da'y Tamperalures Fadaion 24tr averages } & \multicolumn{2}{|c|}{ Hours of Sunstine } & \multicolumn{2}{|c|}{ Eqialent Days } & \multicolumn{2}{|c|}{ Aurreg Humifiy } \\
\hline & $\sqrt{\operatorname{Max} \propto C}$ & Minoc & Dircet Whrne & Dilluso H/me & Tot hours & Maxperday & Simy & Cound & Day & Nons \\
\hline Jancary & 2430 & $\overline{14.40}$ & 150.00 & 6500 & 239.00 & 1151 & 207 & 1023 & 59 & 84 \\
\hline bunery & 2500 & 14.90 & 185.00 & 80.00 & 23300 & 11.72 & 19.88 & 8.12 & 56] & 84 \\
\hline arch & 26.60 & 1620 & 20500 & 85.00 & 26900 & 1222 & 2201 & 8.99 & 56. & 82 \\
\hline Aptil & 28,10 & 18.80 & 275.00 & 115.00 & 223,00 & 13.10 & 21.60 & 8.40 & 56) & 79 \\
\hline Not & 29.70 & 20.90 & 20500 & 125.00 & 27600 & 1349 & 2006 & 10.54 & 59 & 78 \\
\hline Jino & 31.10 & 2310 & 20500 & 125.00 & 255.00 & 1393 & 1830 & 11.70 & 64) & 81 \\
\hline Juty & 31.60 & 23.70 & 255.00 & 125.00 & 270.00 & 1382 & 19.53 & 11.47 & 64) & 81 \\
\hline August & 3210 & 23.80 & 27500 & 115.00 & 261.00 & 13.16 & 19.84 & 11.16 & [3] & 83 \\
\hline Soplanber & 31.10 & 23.70 & 23500 & 8500 & 21200 & 11.98 & 17.70 & 1230 & 68 & 85 \\
\hline Dotabor & 2930 & 21.60 & 185.00 & 80.00 & 200.00 & 11.73 & 17.05 & 13.95 & 64) & 85 \\
\hline Novenber & 2660 & 1810 & 150.00 & 65.00 & 22400 & 11.31 & 1880 & 1020 & 60] & 84 \\
\hline Decomber & 26.10 & 15.10 & 13500 & 55.00 & 20100 & 11.14 & 19.84 & 11.16 & 60) & 85: \\
\hline \multicolumn{4}{|c|}{ BUILOINGDATA } & & \multicolumn{4}{|c|}{ PVPAREI DATA } & \multicolumn{2}{|c|}{ HLLDTYWHATER } \\
\hline \multirow{3}{*}{\multicolumn{3}{|c|}{ 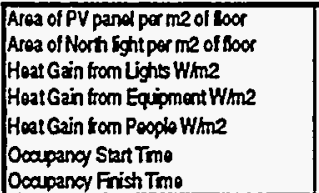 }} & $\begin{array}{r}100 \\
0.15 \\
2000 \\
500\end{array}$ & & \multicolumn{3}{|c|}{ 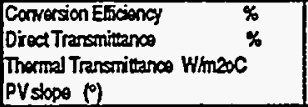 } & $\begin{array}{r}10.00 \\
0.00 \\
0.20 \\
15.00\end{array}$ & EAH & $\begin{array}{r}35 \\
3641\end{array}$ \\
\hline & & & 2000 & & \multicolumn{4}{|c|}{ INTERUL TEHPERATURES } & \multicolumn{2}{|c|}{ HLWDTYSITLER } \\
\hline & & & $\begin{array}{r}8.00 \\
19.00\end{array}$ & & \multicolumn{3}{|c|}{ 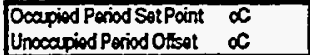 } & $\begin{array}{r}21.00 \\
5.00\end{array}$ & Entraby & $\begin{array}{r}60 \\
47 A 25\end{array}$ \\
\hline
\end{tabular}

CALCULATIOHS OF MEAR EXTERAAL COHDITIOHS

\begin{tabular}{|c|c|c|c|c|c|c|c|c|c|c|}
\hline \multirow[t]{2}{*}{ Month } & \multicolumn{2}{|c|}{ Temperatro $\propto \mathcal{C}$} & \multicolumn{6}{|c|}{ 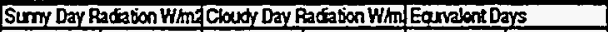 } & \multicolumn{2}{|c|}{ ExtErthaloy $\mathrm{k} / \mathrm{kg}$} \\
\hline & Oocapied & Unocapiod & Oosoioid & Unocapiod & Ocapiod & Unosapiod & Surny & Coudy & Oosupied & Uhocapiod \\
\hline Janeay & 21.83 & 1758 & 305.55 & 3289 & 13557 & 1459 & $20 \pi \pi$ & 1023 & 4908 & 4757 \\
\hline mery & 2249 & 16.15 & 379.85 & 46.68 & 16382 & 20.13 & 19.88 & 8.12 & 4932 & 492 \\
\hline reh & 24.00 & 1854 & 435.25 & 69.18 & 18655 & 29.50 & 201 & 899 & 5321 & 5261 \\
\hline & 25.78 & 21.79 & 48360 & 107.15 & 21066 & 46.67 & 21.60 & 8,10 & 5788 & 5821 \\
\hline$y$ & 2750 & 23.73 & 507.05 & 12639 & 22239 & 5543 & 2046 & 10.54 & 6443] & 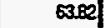 \\
\hline & 20.10 & 25.67 & 49087 & 13728 & 21528 & 6050 & 1830 & 11.70 & 7243 & 7126 \\
\hline & 29.63 & 2624 & 484.76 & 135.17| & 217.00 & 5929 & 1953 & 11.47 & 7456 & 7380 \\
\hline ust & 30.03 & 2547 & 481.83 & 10855 & 20980 & 4728 & 19.84 & 11.16 & 74.60 & 75.78 \\
\hline tomber & 2925 & 2608 & 4484 & 6283 & 10036 & 2689 & 17.70 & 1230 & 7426 & 75.58 \\
\hline lober & 27.39 & 24.08 & 379.53 & 4691 & 163.68 & 2023 & 17.05 & 1395 & 67.19 & 68.60 \\
\hline vartbor & 24.63 & 20.80 & 310.78 & 29.15 & 13789 & 1293 & 12000 & 1020 & 57.00 & 57.57 \\
\hline Decerniber & 2335 & 18.64 & 275.78 & 244 & 11850 & 8.64 & 19:A4 & 11.16 & 5356 & 51.08 \\
\hline \multicolumn{11}{|c|}{ SUHWYOAYTEATBALARCE } \\
\hline \multirow[t]{2}{*}{ Manth } & \multicolumn{2}{|c|}{ Ocapied Perod Whin2 } & & \multirow{2}{*}{\multicolumn{3}{|c|}{ Unocoupiod Period W/m2 }} & \multicolumn{4}{|c|}{ Monty RegurementkWh Fresh Ai Load kWh } \\
\hline & PVnotgin & Intrmis & Oupeal & PVnotgain & & Ororal & Hoaing & $\operatorname{cosing}$ & Heaing & Coofing \\
\hline xery & 3.10 & 2500 & 2810 & -130 & 450 & 320 & 0.00 & 6.77 & 0.00 & 0.66 \\
\hline mery & 4.15 & 2500 & 29.15 & -0.81 & 450 & 3.69 & 0.00 & 6.82 & 0.00 & 0.72 \\
\hline זch & 5.61 & 2500 & 30.51 & -0.69 & 450 & 381 & 0.00 & 7891 & 0.00 & 245 \\
\hline April & 7.17 & 25.00 & 32.17 & -0.211 & 450| & 429 & 0.00 & 824 & 0.00 & 434 \\
\hline$y$ & 848 & 2500 & 33.48 & 0.67 & 450 & 5.17 & 0.00 & 833 & 0.00 & 6.68 \\
\hline 10 & 8.39 & 2500 & 3439 & 0.85 & 450] & 5.45 & 0.00 & 7.68 & 0.00 & 8.78 \\
\hline $\mathbf{u}$ & 8.76 & 25.00 & 34.76 & 120 & 450 & 5.79 & 0.00 & 8.37 & 0.00 & 9.99 \\
\hline & 8.91 & 2500 & 3491 & 122. & 4.50 & 5.72 & 0.00 & 8.51 & 0.00 & 10.35 \\
\hline & 9.10 & 2500 & 34.10 & 0.58 & 450 & 5.08 & 0.00 & 728 & 0.00 & 8.12 \\
\hline & 7.33 & 2500 & 3233 & 023 & 450 & 4.73 & 0.00 & 6.64 & 0.00 & 6A7 \\
\hline & 4.97 & 25.00 & 29.97 & -0.47 & 450 & 4.03 & 0.00 & 7.05 & 0.00 & 3.64 \\
\hline 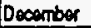 & 3.84 & 25.00 & 2884 & -0.70 & 450 & 3.80 & 0.00 & 6.78 & 0.00 & 234 \\
\hline
\end{tabular}

CLOUDY DAY HEAT BALANCE

\begin{tabular}{|c|c|c|c|c|c|c|c|c|c|c|}
\hline \multirow{2}{*}{ Month } & \multicolumn{3}{|c|}{ Ooapoid Period W/m2 } & \multicolumn{3}{|c|}{ Unocoupiad Penod Whn2 } & \multicolumn{4}{|c|}{ 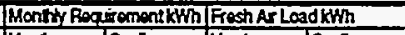 } \\
\hline & PY notgin & Interneds & Oworal & PVmotgin & Intrmals & Oerel & Heaing & Cooling & Hoaing & $\cos \sqrt{n} g$ \\
\hline Januery & 1.68 & $\mathbf{4 5 0 0}$ & 46.68 & $-0,47$ & 450 & 400 & 0.00 & 5.35 & 0.00 & 0.53 \\
\hline ary & 233 & 40.00 & 4233 & -0.60 & 450 & 3.90 & 0.00 & 3.88 & 0.00 & 0.30 \\
\hline March & 352 & 35,00 & 3852 & -0.37 & 450 & 4.13 & 0.00 & 388 & 0.00 & 100 \\
\hline Appi & 4.87 & 30.00 & 34.87 & -0.18 & 450 & 432 & 0.00 & 3.44 & 0.00 & 1.69 \\
\hline May & 6.09 & 2500 & 31.09 & 0.07 & 450 & 457 & 0.00 & 3.56 & 0.00 & 3AA \\
\hline $\operatorname{Non} \theta$ & 7.07 & 2500 & 3207 & 029 & 450 & 4.79 & 0.00 & 454 & 0.00 & 5.22 \\
\hline Juty & 7.43 & 25.00 & 3243 & 0.65 & 450 & 5.15 & 0.00 & 455 & 0.00 & 5.86 \\
\hline Augast & 7.63 & 2500 & 3203 & 0.70 & 4.50 & 520 & 0.00 & 4.45 & 0.00 & 5.82 \\
\hline Soptomber & 6.96 & 30.00 & 35.96 & 028 & 4.50 & 4.78 & 0.00 & 5.37 & 0.00 & 6.34 \\
\hline lotaber & 5.52 & 3500 & 4052 & 0.00 & 450 & 450 & 0.00 & 653 & 0.00 & 529 \\
\hline November & 351 & 40.00 & 4351 & -228 & 450 & 422 & 0.00 & 5.04 & 0.00 & 1.87 \\
\hline Decomber & 252 & 45.00 & 47.52 & -0.37 & 450 & 4.13 & 0.00 & 5.95 & 0.00 & 131 \\
\hline
\end{tabular}

OVERALL ERERGY REQURRELENTS AND PRODUCTOW

\begin{tabular}{|c|c|c|c|c|c|c|c|}
\hline \multirow[t]{2}{*}{ Month } & \multicolumn{2}{|c|}{ Coding Consungion } & \multicolumn{2}{|c|}{ Heating Consernotion } & \multirow{2}{*}{$\begin{array}{l}\text { Fans } \\
\text { twh }\end{array}$} & \multirow{2}{*}{$\begin{array}{l}\text { Ligring and } \\
\text { Pomer Kthth }\end{array}$} & \multirow{2}{*}{$\begin{array}{l}\text { Tobl kWh } \\
\text { Consemad }\end{array}$} \\
\hline & $1 / 000$ & kWh Bectic & 1 loop & kMh Eoctic & & & \\
\hline Januay & 029 & 3.75 & 022 & 0.00 & 2.52 & 4.68 & 11.05 \\
\hline Fobruary & 029 & 3.35 & 022 & 0.00 & 237 & 3.60 & 931 \\
\hline Neasch & 0.29 & 4.38 & 022 & 0.00 & $2 \curvearrowleft$ & 3.53 & 10.54 \\
\hline Apet & 029 & 5.06 & - 022 & 0.00 & 253 & 297 & 10.55 \\
\hline $1 \times b y$ & 020 & 6.40 & 022 & 0.00 & 202 & $26 A$ & 11.66 \\
\hline Jung & 0.29 & 7.61 & 022 & 0.00 & 253 & 2.55 & 1269 \\
\hline Juty & 029 & 822 & 022 & 0.00 & 2.02 & 264 & 1347 \\
\hline Alugust & 0.29 & 8.33 & 022 & 0.00 & 202 & 2.64 & 1358 \\
\hline Seprember & 0.29 & 8.03 & 022 & 0.00 & 253 & 3.17 & 13.73 \\
\hline Ootcoser & 0.29 & 7.12 & 022 & 0.00 & 262 & 4.03 & $137 \pi$ \\
\hline November & 022 & 5.03 & 0.2 & 0.00 & 253 & 4.08 & 11.64 \\
\hline Decernber & 029 & 4.68 & 022 & 0.00 & 2.02 & 4.87 & 1217 \\
\hline TOTAL/m2 & & 71.95 & & 0.00 & 30.84 & 4138 & 144.17 \\
\hline
\end{tabular}


OPTION 4: $\quad$ PV ROOF MONTORS

\begin{tabular}{|c|c|c|c|c|c|c|c|c|c|c|}
\hline $\begin{array}{l}\text { Chy } \\
\text { Crmato }\end{array}$ & $\begin{array}{l}\text { Chicago } \\
\text { Tomperabon }\end{array}$ & oninental & $\begin{array}{l}\text { Longitdo } \\
\text { Lafiuds } \\
\text { Arthodo }\end{array}$ & $\begin{array}{r}41.78 \\
87.75 \\
185.00\end{array}$ & & $\begin{array}{l}\text { Droct Padaf } \\
\text { Dituso Pada }\end{array}$ & $\begin{array}{l}\text { ion Factor } \\
\text { lion Factor }\end{array}$ & $\begin{array}{l}0.50 \\
1.10\end{array}$ & & \\
\hline WEATRER & DATA & & & & & & & & & \\
\hline Manth & Typical Dajy T & Tomperatres & Radaion $24 r$ & xaverages II & Hours of Suns & $\sin \theta$ & Egivalent Da & & Avereostum & ionty $\%$ \\
\hline & $\operatorname{Max} \propto C$ & Mnoc & Diroctw/n2 & D茄 1 so W/mad & Toy hars & Maxpor day & Surny & Caosy & Day & Nint \\
\hline Varuery & 0.50 & -720 & 65.00 & 40.00 & 12500 & 10.00 & 1250 & 1850 & 70 & 80 \\
\hline Fobrery & 1.50 & -630 & 130.00 & 5500 & 141.00 & 10.48 & 1344 & 1456 & 67 & 78 \\
\hline March & GAO & -1.70 & 980.00 & 7500 & 206.00 & 1278 & 16.12 & 1468 & 61. & $\pi$ \\
\hline pio & 14.10 & 4.70 & 250.00 & 100.00 & 206.00 & 1248 & 1650 & 1350 & 58 & 74 \\
\hline Hay & 20.50 & 1050 & 20000 & 120.00 & 275.00 & 1454 & 1891 & 1200 & 55 & 75 \\
\hline Juno & 2640 & 1540 & 305.00 & 125.00 & 307.00 & $155 t$ & 1980 & 1020 & 56 & $\pi$ \\
\hline Juty & 2890 & 1950 & 20000 & 120.00 & 310.00 & 1389 & 2232 & 8.68 & 53 & $\pi$ \\
\hline Augst & 2800 & 1880 & 250.00 & 100.00 & 285.00 & 13.13 & 21.70 & 930 & 56 & 81 \\
\hline Seppomber & 2380 & 18.10 & 180.00 & 7500 & 246.00 & 1242 & 18,80 & 10.20 & 53 & 81 \\
\hline October & 1740 & 820 & 130.00 & 55,00 & 21400 & 11.13 & 1922 & 11.78 & 54 & 78 \\
\hline Novenbex & 810 & 030 & 8500 & 4000 & 135.00 & 1000 & 1350 & 16.50 & $\infty$ & 78 \\
\hline Decorrber & 2.10 & -530 & 70.00 & 35,00 & 115.00 & 10.00 & 1150 & 1950 & 69 & 80 \\
\hline BU Dikg & ATA & & & & PVPXRED & ATR & & & HUDDTrW & INIER \\
\hline $\begin{array}{l}\text { Area of PV p } \\
\text { Area of Nort } \\
\text { Heat Gain tr } \\
\text { Heat Gain tr }\end{array}$ & $\begin{array}{l}\text { and por m2 of } \\
\text { hight por me of } \\
\text { on Lights W/m2 } \\
\text { on Eqipnent }\end{array}$ & $\begin{array}{l}\text { loor } \\
\text { flbor } \\
2 \\
\text { Whon }\end{array}$ & \begin{tabular}{r|}
1.00 \\
0.15 \\
20.00 \\
5.00
\end{tabular} & & $\begin{array}{l}\text { Consersion E } \\
\text { Oirect Transm } \\
\text { Thxmal Tren } \\
\text { PV stope (9) }\end{array}$ & $\begin{array}{l}\text { Sicioncy } \\
\text { nimanos } \\
\text { sitanco Wh }\end{array}$ & $\ln _{2 x} x^{\%}$ & \begin{tabular}{r|}
10.00 \\
0.00 \\
0.20 \\
15.00
\end{tabular} & Entraby & $\begin{array}{r}35 \\
36,41\end{array}$ \\
\hline Hoal Gain fr & an Psoplo W/ne & & 2000 & & IFIERNAL T & EMPEATUR & & & HUTSTISS & ATIER \\
\hline $\begin{array}{l}\text { Oocapancy } 5 \\
\text { osapancy } \mathrm{F}\end{array}$ & $\begin{array}{l}\text { Sart Tme } \\
\text { Erish Tme }\end{array}$ & & $\begin{array}{r}9.00 \\
12.00\end{array}$ & & $\begin{array}{l}\text { Ocapiod Pon } \\
\text { Unocapiod P }\end{array}$ & $\begin{array}{l}\text { nod Sol Point } \\
\text { Period Otsot }\end{array}$ & $\begin{array}{l}\infty \\
\infty\end{array}$ & $\begin{array}{r}21.00 \\
5.00\end{array}$ & $\begin{array}{l}\text { Sort } \\
\text { Entraby }\end{array}$ & $\begin{array}{r}60 \\
47 A 25 \\
\end{array}$ \\
\hline CALCLAT & IONS OFMEAY & MEXTERMLL & coldomos's & & & & & & & \\
\hline Manth & Temperalure & & Sim Day & afation W/nt & Clouts Day & 2acion W/n & Equivans Da & ays & Ext Entaloyk & $\mathrm{k} / \mathrm{kg}$ \\
\hline & Oocapiod II & thocapiod & Doaniod I & Uhocapiod & Ocapiod & Chocapiod & Sinny & County & Doriond & Uhoconind \\
\hline $\operatorname{Santany}$ & -135 & 4.69 & 207.60 & 0.00 & 86.00 & 0.00 & 1250 & 1850 & 3.56 & -0.55 \\
\hline Fabrery & -0.45 & -3.79 & 297.10 & 10.07 & 125.82 & 4A1 & 134 & 1458 & 4.78 & 0.54 \\
\hline Warch & 438 & 0.90 & 33336 & 66.17 & 140.85 & 2796 & 16.12 & 1488 & 1207 & 780 \\
\hline Appi & 11.75 & 7.72 & 451.75 & 80.18 & 182.23 & 34.12 & 1650 & 1350 & 2503 & 20.36 \\
\hline Mry & 1808 & 13.75 & 457.14 & 14833 & 188.04 & 6426 & 1891 & 1200 & 3834 & 3437 \\
\hline Juno & 23.65 & 1894 & 4868 & 176.51 & $183 / 9$ & 76.08 & 19800 & 1020 & 5231 & 4888 \\
\hline Why & 2655 & 2252 & 472.66 & 13296 & 207.36 & 5760 & 2232 & 8.68 & 5817 & 59.53 \\
\hline August & 25.70 & 21.76 & 429,43 & 86.12 & 182.74 & 40.90 & 21.70 & 830 & 57.68 & 59.08 \\
\hline Sepmonber & 21.38 & 1722 & 34288 & 5937 & 144.88 & 2509 & 19.80 & 1020 & 4523 & 45,46 \\
\hline October & 15.10 & 11.16 & 27052 & 21.92 & 118.55 & 9.60 & 1922 & 11.78 & 31.4 & 29.05 \\
\hline Norember & 638 & 290 & 207.60 & 0.00 & 96.00 & 0.00 & 1350 & 16.50 & 15.78 & 1143 \\
\hline Decomber & 0.25 & -289 & 176.40 & 0.00 & 84.00 & 0.00 & 1150 & 1850 & 6.03 & 1.55 \\
\hline SLWTYDAY & HEAT BALAY & $\mathrm{NCE}$ & & & & & & & & \\
\hline Man & Oocopiod Penio & iodW/m2 & & Unocaraiod Pe & PeriodW/n2 & & Morthy Plog: & sementkWh & Frosh A Load & IdkWh \\
\hline & PVnotain & intends & Ooral & PVnelgain I & Intrmils & Oraral & Healing & $\cos \mathrm{ing}$ & Hoaling & $\cos x$ \\
\hline Janumy & -1278 & 2500 & 122 & -1345 & 450 & -895 & -157 & 1.53 & .787 & 0.00 \\
\hline breny & -11.53 & $25 \infty$ & 13.47 & -1278 & 450 & -828 & -156 & 1.81 & -8.16 & 0.00 \\
\hline wh & $\$ 01$ & 2500 & 1699 & -926 & 450 & -4.76 & -1.07| & 271 & .754 & 0.00 \\
\hline Apri & -222 & 2500 & 2278 & 471 & 4.50 & -0.21 & -0.05 & 3.76 & 3.51 & 0.00 \\
\hline by & 194 & 2500 & 2694 & -184 & 450 & 2.66 & 0.00 & 580 & 0.00 & $0 \times 0$ \\
\hline Jino & 5,49 & 2500 & 30.49 & -0.94 & 450 & 356 & 0.00 & 7.02 & 0.00 & 1.86 \\
\hline hut & 7.63 & 2500 & 3263 & -0.05 & 450 & $4 M$ & 0.00 & 8.67 & 0.00 & 4.61 \\
\hline Augst & 6.66 & 2500 & 31.66 & -0.33 & 4.50 & 4.17 & 0.00 & 8.14 & 0.00 & 427 \\
\hline Soprenber & 3.12 & 2500 & 28.12 & -0.33 & 450 & 4.17 & 0.00 & 6.72 & 0.00 & 0.00 \\
\hline Octabor & -156 & 2500 & 2344 & -296 & 450 & 151 & 0.00 & 492 & .134 & 0.00 \\
\hline Norember & -7.76 & 2500 & 1724 & -851 & 450 & -4.01 & -078 & 233 & -535 & 0.00 \\
\hline Docember & -1201 & 25.00 & 1209 & -1230 & 450 & -7.80 & -126 & 149 & -6.71 & 0.00 \\
\hline CLOWOYDI & IYHEATBALA & AMCE & & & & & & & & \\
\hline Manth & poapiod Peiv & iodW/m2 & & Unocapiod Pe & IH/n2 & & Honth Rogi & ntkWh & Afload & dkWh \\
\hline & PVnat gain & Intrmals & Overal & PVnatgain & Intmals & Orerl & Hoing & $\operatorname{cosing}$ & Heaing & $\operatorname{cosing}$ \\
\hline xery & -13.72 & 45.00 & 3128 & -1345 & 450 & -8.85 & -232 & 5.78 & -11.64 & 0.00 \\
\hline bury & -1289 & 40,00 & 27.11 & .1283 & 450 & -833 & -170 & 395 & 884 & 0,0 \\
\hline rach & -9.02 & 3500 & 2538 & .8 .58 & 450 & 5.08 & -1.06 & 3.78 & -606 & 0.00 \\
\hline it & +40 & 30.00 & 2560 & 500 & 450 & -0.59 & -0.11 & 346 & -255 & 0.00 \\
\hline May & -024 & 2500 & 24.76 & -0.98 & 450 & 357 & 0.00 & 3.60 & 0.00 & 0.00 \\
\hline Jins & 3.35 & 2500 & 2835 & -0.38 & 450 & 4.12 & 0.00 & 348 & 0.00 & 0.96 \\
\hline Juty & 535 & 2500 & 30.35 & -0.15 & 450 & 435 & 0.00 & 3.16 & 0.00 & 1.78 \\
\hline Augs & 459 & 25.00 & 29.50 & -0.25 & 450 & 425 & 0.00 & 331 & 0.00 & 183 \\
\hline amber & 1.46 & 3000 & $3 t A 6$ & -0.52 & 4.50 & 3.88 & 0.00 & 3.76 & 0.00 & 0.00 \\
\hline cober & -284 & 35,00 & 3216 & 307 & 450 & 1,13 & 0.00 & 4.02 & -1.13 & 0.00 \\
\hline Vaveriber & 270 & 40,00 & 3130 & -851 & 450 & -4.01 & -0.93 & 5.16 & 6.54 & 0.00 \\
\hline Docomber & -1278 & 4500 & 322 & -1230 & 450 & -7.80 & -213 & 628 & -1138 & 0.00 \\
\hline
\end{tabular}

OVERALI ERERGY FEQLERERERIS ADD PROOUCTOH

\begin{tabular}{|c|c|c|c|c|c|c|c|}
\hline \multirow[t]{2}{*}{ Month } & \multicolumn{2}{|c|}{ Cooding Constmpion } & \multicolumn{2}{|c|}{ Healing Consumption } & \multirow{2}{*}{$\begin{array}{l}\text { Fans } \\
\text { kWh }\end{array}$} & \multirow{2}{*}{$\begin{array}{l}\text { Lighting and } \\
\text { Pomor ikh }\end{array}$} & \multirow{2}{*}{$\begin{array}{l}\text { Toral kWh } \\
\text { Consemed }\end{array}$} \\
\hline & 1000 & Khm Bectic & 11000 & Whap Boctic & & & \\
\hline Janxay & 0.00 & 0.00 & 022 & 520 & 232 & 6.34 & 1386 \\
\hline Fobruary & 0.00 & 0.00 & 022 & 450 & 2.10 & 456 & $\$ 1.16$ \\
\hline March & 0.00 & 0.00 & 0.22 & 3.60 & 232 & 4.12 & 10.16 \\
\hline Appi & 0.00 & 0.00 & 022 & 1,49 & 225 & 323 & 6.97 \\
\hline Mey & 029 & 2.60 & 022 & 0.00 & 232 & 2.64 & 7.64 \\
\hline Jine & 0.29 & 381 & 0.22 & 0.00 & 225 & 255 & 8.60 \\
\hline Juty & 0.29 & 521 & 0.22 & 0.00 & 232 & 2.64 & 10.17 \\
\hline August & 0.29 & 5.01 & 022 & 0.00 & 232 & 2.64 & 9.97 \\
\hline Septomber & 029 & 3.00 & 0.22 & 0.00 & 225 & 3.06 & 830 \\
\hline Oateber & 0.29 & 256 & 0.22 & 0.66 & 232 & 381 & 9.35 \\
\hline Norombor & 0.00 & 0.00 & 022 & 3.02 & 225 & 5.03 & 1020 \\
\hline Decombar & 0.00 & 0.00 & 022 & 4.77 & 232 & 6.54 & 1365 \\
\hline TOTAL/m2 & & 2226 & & 23.33 & 27.35 & 47.14 & 120.08 \\
\hline
\end{tabular}


OPTION 4: $\quad$ PV ROOF MONITORS

(R36 equiv. insulation)

STIE DATA

\begin{tabular}{|c|c|c|c|c|c|}
\hline aty & $\begin{array}{l}\text { Cricinas } \\
\text { Tremperesw continontal }\end{array}$ & $\begin{array}{l}\text { Longivide } \\
\text { Larude } \\
\text { Astadese }\end{array}$ & $\begin{array}{r}39.15 \mathrm{~N} \\
8452 \mathrm{~W} \\
23200 \mathrm{~m}\end{array}$ & 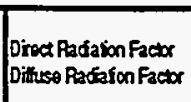 & $\begin{array}{l}0.50 \\
1.10\end{array}$ \\
\hline
\end{tabular}

\section{WEATHERDATA}

\begin{tabular}{|c|c|c|c|c|c|c|c|c|c|c|}
\hline \multirow[t]{2}{*}{ Month } & \multicolumn{2}{|c|}{ Typical Dary Terrocraires } & \multicolumn{2}{|c|}{ Radalion 24 tr averages } & \multicolumn{2}{|c|}{ Hours of Sunstine } & \multicolumn{2}{|c|}{ Equalent Days } & \multicolumn{2}{|c|}{ Avorage Henicity $\%$} \\
\hline & $\operatorname{Max} \propto C$ & Mnoc & DrectW/m2 & Ditheso H/me & Total hars & Maxporday & Surny & Couny & Day & Nigit \\
\hline January & 620 & 330 & 8500 & 40.00 & 108.00 & 10.00 & 10.80 & 2020 & 68 & 83 \\
\hline abreay & 6.30 & -200 & 130,00 & 5500 & 129.00 & 1024 & 1260 & 1540 & 64 & 81 \\
\hline arch & 11.10 & 0.70 & 180.00 & 75.00 & 17300 & 11.16 & 1550 & 1550 & 57) & 79 \\
\hline n] & 1800 & 6.60 & 25000 & 10000 & 201.00 & 11.96 & 1680 & 1320 & 53) & 76 \\
\hline ey & 2380 & 11.90 & 290000 & 120.00 & 241.00 & 1274 & 1891 & 1209 & 53 & 78 \\
\hline no & 29800 & 1720) & 325.00 & 125.00 & 305.00 & 14.73 & 20.70 & 830 & 50 & 80 \\
\hline b & 3080 & 19.10 & 20000 & 120.00 & 32200 & 14.43 & 2220 & 8.58 & 52 & 82 \\
\hline gust & 3020 & 18.30 & 250.00 & 100.00 & 291.00 & 134t & 21.70 & 830 & 52 & 86 \\
\hline prember & 2680 & $\cdot 1420$ & 100.00 & 7500 & 254.00 & 1245 & 20.40 & 8.60 & 50 & 86 \\
\hline atber & 2050 & 8.20 & 130.00 & 5500 & 213.00 & 1126 & 1891 & 1209 & 61 & es \\
\hline overnber & 1180 & 220 & 8500 & 4000 & 145.00 & 10.51 & 1380 & 1620 & 60 & 81 \\
\hline Docember & 5.90 & -230 & 70,00 & 3500 & 126.00 & 10.16 & 1240 & 18.60 & 68 & 80 \\
\hline \multicolumn{4}{|c|}{ BU DIFGDATA } & & \multicolumn{4}{|c|}{ PVPAEEDATA } & \multicolumn{2}{|c|}{ HUL DTYWHTER } \\
\hline \multirow{5}{*}{\multicolumn{3}{|c|}{ 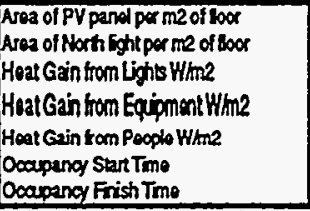 }} & & & \multirow{3}{*}{\multicolumn{3}{|c|}{ 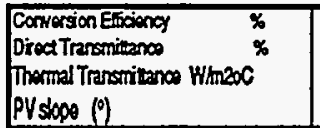 }} & & \multirow{3}{*}{ Entrapy } & 35 \\
\hline & & & $\begin{array}{c}0.15 \\
20,00\end{array}$ & & & & & $\begin{array}{r}0.00 \\
020\end{array}$ & & 641 \\
\hline & & & 500 & & & & & 1500 & & $4 T=8$ \\
\hline & & & 20.00 & & \multicolumn{4}{|c|}{ INTEFUL TELPERATLRES } & \multirow[b]{2}{*}{ Entraby } & 60 \\
\hline & & & $\begin{array}{r}9.00 \\
18,00\end{array}$ & & \multicolumn{3}{|c|}{$\begin{array}{l}\text { Deapiod Pariod Sot Point } \propto C \\
\text { Unooupiod Period Otsol }\end{array}$} & $\begin{array}{r}21.00 \\
5.00\end{array}$ & & 47,125 \\
\hline \multicolumn{11}{|c|}{ CALCULATIOHS OF HEXHEXTEPULL COHDITOHS } \\
\hline \multirow[t]{2}{*}{ Manth } & \multicolumn{2}{|c|}{ Temporatro oc } & Sumy Day R2 & deation $Y / m$ I & Cloudy Day R & abon $\mathrm{HIm}$ & Earvalont Da & & ExtEntraloy & $\mathrm{k} / \mathrm{kg}$ \\
\hline & Ocapiod & Thocapied & Oocaind & thocapiod & Coapiad & Ihocapied & Sumy & Coudy & Ooonpied & IThocaloisd \\
\hline andary & 383 & -025 & 207.60 & 0.00 & 86.00 & 0.00 & 10.80 & 2020 & 11.96 & 636 \\
\hline breay & 4.00 & 0.06 & 29420 & 5,00 & 12893 & 219 & 1260 & 15,40 & 11.79 & 6.71 \\
\hline $\operatorname{arch}$ & 8.50 & 4.04 & 381.68 & 31.66 & 16127 & 1338 & 1550 & 1550 & 1891 & 13.69 \\
\hline oi & 15.15 & 1026 & 471,40 & 66.14 & 200.60 & 28.14 & 1680 & 1320 & 3124 & 2631 \\
\hline ay & 2083 & 15.73 & 521.63 & 10226 & 225.98 & 4430 & 1891 & 12009 & 4393 & 40.35 \\
\hline no & 2590 & 20.93 & 47237 & 159.74 & 203.51 & 6885 & 20.70 & 8.30 & 57.64 & 55.01 \\
\hline Wy & 2788 & 2286 & 460.82 & 145.70 & 199.63 & 63.12 & 2232 & 8.68 & 61.04 & 6303 \\
\hline ings & 2723 & 2213 & 420.58 & 1024 & 178.97 & 4359 & 21.70 & 9.30 & 5933 & 0255 \\
\hline eplenber & 2365 & 1825 & 34.14 & 59.90 & 14457 & 25.31 & 2040 & 9.50 & 4924 & 5028 \\
\hline tober & 1743 & 12.15 & 26740 & 24.14] & 117.19 & 10.58 & 1891 & 1209 & 3398 & 3294 \\
\hline oromber & 8,40 & 520 & 19758 & 7.16 & 81,37 & 331 & 13800 & 1620 & 2121 & 16A1] \\
\hline December & 3.25 & 0.34 & 173.60 & 2.00 & 8267 & 0.95 & 1240 & 18.60 & 1200 & 727 \\
\hline DWWY & 397 & & & & & & & & & \\
\hline Annth & Conpied Pen & nodW/m2 & & $\operatorname{arosdp}$ & Whit & & YBoga & $\mathrm{k} k \mathrm{~m}$ & FreshÁ Loo & dKWh \\
\hline & PVnotgain & Internds & Oueral & PV mot gain & Intonds & Toveral & Hoaing & Cooing & Heaing & $\cos i n g$ \\
\hline nuxy & $-9,42$ & 25.00 & 1558 & -1056 & 450 & 6.05 & -0.82 & 1.68 & 5.07 & 0.00 \\
\hline bruey & -858 & 2500 & 16,12 & -1032 & 450 & -582 & -1.03 & 2.07 & -596 & 0.00 \\
\hline berch & 4.92 & 2500 & 20.08 & -751 & 450 & 301 & -0.55 & 3.11 & 521 & 0.00 \\
\hline$x i$ & 0.16 & 2500 & 25,16 & 3.17 & 450 & 1.33 & 0.00 & 454 & -1.67 & 0.00 \\
\hline $\boldsymbol{y}$ & 427 & 2500 & 2027 & -0.04 & 1450 & 3.56 & 0.00 & 6.48 & 0.00 & 0.00 \\
\hline$\infty$ & 7.15 & 25.00 & 3215 & -0.87 & 450 & 3.63 & 0.00 & 7.71 & 0.00 & 406 \\
\hline 中 & 834 & 2500 & 3334 & -0.82 & 450 & 3.68 & 0.00 & 8.59 & 0.00 & 583 \\
\hline & 758 & 25.00 & 3258 & -0.57 & 450 & 393 & 0.00 & 825 & 0.00 & 498 \\
\hline septernter & 460 & 25.00 & 29.60 & -0.63 & 450 & 387 & 0.00 & 7.14 & 0.00 & 0.71 \\
\hline oet & -0.08 & 25.00 & 2492 & -230 & 450 & 220 & 0.00 & 5.30 & 0.00 & 0,00 \\
\hline Novertiber & -580 & 25.00 & 19.12 & -690 & 450 & -240 & -0.46 & 264 & +1.03 & 0.00 \\
\hline lecentiber & -9.69 & 25.00 & 1531 & -10.16 & 450 & 5.66 & -0.98 & 180 & -5889 & 0.00 \\
\hline
\end{tabular}

CLOLOY DAY HEAT BALAYCE

\begin{tabular}{|c|c|c|c|c|c|c|c|c|c|c|c|}
\hline \multirow{3}{*}{ Month } & \multirow{2}{*}{\multicolumn{2}{|c|}{$\begin{array}{l}\text { Oocapiod PenodW/m2 } \\
\text { PVnel gain Intmats }\end{array}$}} & \multirow[b]{2}{*}{ Operd } & \multicolumn{2}{|c|}{ Unocapiod Period W/m2 } & \multirow[b]{2}{*}{ Ooend } & \multicolumn{5}{|c|}{ 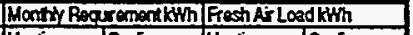 } \\
\hline & & & & PYnot gain I & Internals & & Heaing & Cooing | & Healing & cooing & \\
\hline & -1036 & 45.00 & 34.64 & -10.56 & 4.50 & -6.06 & -1.71 & 7.00 & -9.48 & & 0.00 \\
\hline Fobruary & -9.97 & 40.00 & 30.03 & -10.34 & 450 & -584 & -126 & 4.63 & .728 & & 0.00 \\
\hline March & $-67 n$ & 35,00 & 2823 & -7.66 & 450 & -3.16 & -0.00 & 4.38: & -521 & & 0.00 \\
\hline fapri & -212 & 30.00 & 2788 & 240 & 450 & 1.01 & 0.00 & 3.87] & -131 & & 0.00 \\
\hline May & 1.78 & 25,00 & 26.78 & $-1 / 13$ & 4.50 & 3.07 & 0.00 & 3.75 & 0.00 & & 0.00 \\
\hline Juse & 450 & 25.00 & 29.90 & -0.12 & 450 & 438 & 0.00 & 335 & 0.00 & & 1.22 \\
\hline futy & 6.15 & 25.00 & 31.15 & 0.11 & 450 & 4.61 & 0.00 & 326) & 0.00 & & 227 \\
\hline fungust & 555 & 25.00 & 30.55 & 0.01 & 450 & 451 & 0.00 & 343 & 0.00 & & 2.13 \\
\hline Scplentiber & 294 & 30.00 & 3204 & .092 & 450 & 358 & 0.00 & 3.64 & 0.00 & & 033 \\
\hline Detroer & -134 & 35.00 & 32.68 & -241 & 4.50 & 200 & 0.00 & 4.42 & 0.00 & & \\
\hline vernber & $-6 \pi$ & 40.00 & 3323 & -694 & 450 & -244 & -0.55 & 5.38 & -4.73 & & 0.00 \\
\hline Docantiber & -1045 & 45.00 & 34.55 & -10.17| & 450 & -5.67 & $-1 / 48$ & 6.43 & -872 & & 0.00 \\
\hline
\end{tabular}

OVERKII ERERGY REQURREDENTS A PD PROOUUCTIOH

\begin{tabular}{|c|c|c|c|c|c|c|c|c|}
\hline \multirow[t]{2}{*}{ Month } & \multicolumn{2}{|c|}{ Coding Consumpion } & \multicolumn{3}{|c|}{ Heasing Consumosion } & \multirow{2}{*}{$\begin{array}{l}\text { Fans } \\
\text { Kuth }\end{array}$} & \multirow{2}{*}{$\begin{array}{l}\text { Lighing and } \\
\text { Power Kwh }\end{array}$} & \multirow{2}{*}{$\begin{array}{l}\text { Tot then } \\
\text { Consined }\end{array}$} \\
\hline & 11000 & Axhloctic & $1 / 000$ & & Whectic & & & \\
\hline Janeary & 0.00 & 0.00 & & 022 & 3.82 & 245 & 6.68 & 1294 \\
\hline Fobruary & 0.00 & 0.00 & & 02 & 3.45 & 221 & 4.59 & 10.35 \\
\hline March & 0.00 & 0.00 & & 022 & 261 & $2 A 5$ & 4.19 & 925 \\
\hline Apri & 020 & $2 A 0$ & & 0.22 & 0.66 & 237 & 321 & 8.64 \\
\hline Hay & 029 & 292 & & 022 & 0.00 & 245 & 2.64 & 8.01 \\
\hline Jene & 020 & 4.84 & & 022 & 0.00 & 237 & 255 & 9.76 \\
\hline Juty & 029 & 5.70 & & 022 & 0.00 & 245 & 2.64 & 10.79 \\
\hline August & 029 & 5.36 & & 022 & 0.00 & 245 & 264 & 10.45 \\
\hline Soptember & 029 & 338 & & 022 & 0.00 & 237 & 3.03 & 8.78 \\
\hline Oacabor & 029 & 278 & & 022 & 0.00 & $2 A 5$ & 3.84 & 807 \\
\hline Novention & 0.00 & 0.00 & & 022 & 217 & 237 & 488 & 958 \\
\hline Docomber & 0.00 & 0.00 & & 0.22 & 3.70 & 245 & 6.36 & 1258 \\
\hline TOTALm2 & & 2739 & & & 16,49 & 2883 & 47.42 & 120.14 \\
\hline
\end{tabular}


STE DATA

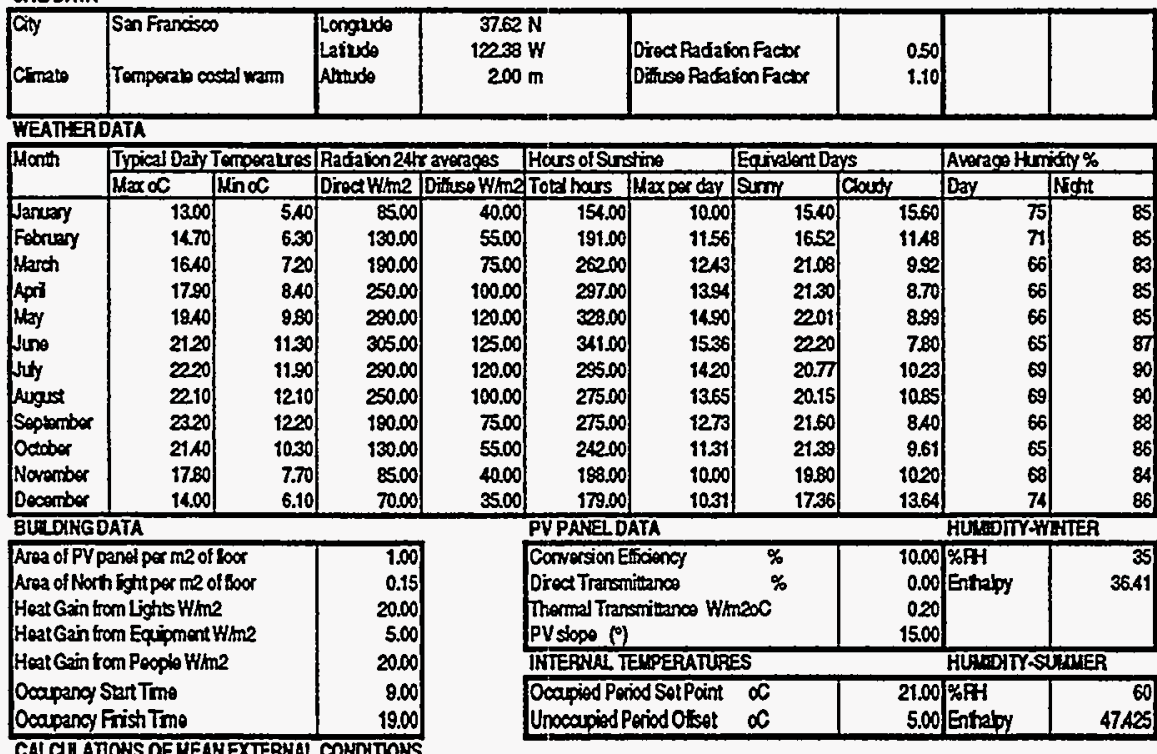

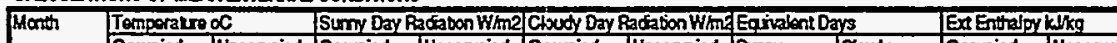

\begin{tabular}{|c|c|c|c|c|c|c|c|c|c|c|}
\hline & & & & & & & & & & \\
\hline & Oosupiod & Whocociod & Oocaioid & Uhocapied & Docapied & Thoocariod & Simy & Coondy & Oocaniod & Thoocioiod \\
\hline Janery & 11.10 & 734 & 207.60 & 0.00 & 96.00 & 0.00 & 15.40 & 15.60 & 2801 & 2252 \\
\hline Faloreary & 1260 & 9.00 & 26051 & 29.06 & 114.17 & 1274 & 1652 & 11,48 & 30.55 & 25.19 \\
\hline March & 14.10 & 10.16 & 342.75 & 5946 & 1488 & 25.13 & 21.08 & 8.92 & 3271 & 2753 \\
\hline Apri & 1553 & 11,45 & 40448 & 113.94 & 172.12 & 4848 & 2130 & 8.70 & 3508 & 31.15 \\
\hline Nos & 17.00 & 1280 & 46.11 & 15628 & 19326 & 67.67 & 2200 & 8.99 & 39.69 & 34.84 \\
\hline fune & 1873 & 1448 & 453.11 & 173.19 & 185.31 & 74.78 & 220 & 780 & 4367 & 39.69 \\
\hline buy & 19.03 & 1521 & 468.06 & 14053 & $202 \pi$ & 6068 & 20.77 & 1023 & (17.62) & 4264 \\
\hline August & 18.60 & 1531 & 41326 & 107.67 & 17585 & 4580 & 20.15 & 10.85 & 4755 & 224 \\
\hline Soplariber & 20,15 & 15.74 & 334.60 & 6528 & 14138 & 2758 & 21.60 & 840 & 4859 & 4354 \\
\hline Databor & 1860 & 1388 & 266.23 & 24.98 & 116.57 & 10.95 & 2139 & 8.51 & 4342 & 37.72 \\
\hline Norember & 1528 & 10.95 & 207.60 & 0.00 & 9600 & 0.00 & 1980 & 1020 & 36.10 & 29.66 \\
\hline Decomber & 1203 & 8.64 & 177.08 & 3.80 & 81,47 & 1.81 & 1736 & 1364 & 2994 & 2450 \\
\hline
\end{tabular}

\begin{tabular}{|c|c|c|c|c|c|c|c|c|c|c|}
\hline \multirow[t]{2}{*}{ Manth } & \multicolumn{3}{|c|}{ Ocaniad PariodWhr2 } & \multicolumn{3}{|c|}{ Unocapiad Paid W/m2 } & \multicolumn{4}{|c|}{ Monthy RegirementkWh Fresh Air Load KWh } \\
\hline & PVnotgin & momels & Jowal & PYnotgain & Insme's & Daner I & Heaing & Cosing & Heaing & Cooing \\
\hline Januery & 4.69 & 25.00 & 20.31 & -530 & 450 & -0.80 & -0.17 & 3.13 & -248 & 0.00 \\
\hline Fabruary & 327 & 2500 & 21.73 & $-4,31$ & 450 & 0.18 & 0.00 & 3.63 & -186 & 0.00 \\
\hline Manch & .161 & 2500 & 2330 & 330 & 450 & 120 & 0.00 & 520 & -150 & 0.00 \\
\hline Apri & -0.16 & 2500 & 2484 & -200 & 450 & 250 & 0.00 & 6.04 & -0.14 & 0.00 \\
\hline May & 1.15 & 2500 & 2615 & -180 & 450 & 2.70 & 0.00 & 6.59 & 0.00 & 0.00 \\
\hline Lino & 233 & 2500 & 2733 & -1.15 & 4.50 & 3.35 & 0.00 & 7.11 & 0.00 & 0.00 \\
\hline Juty & 3.04 & 25.00 & 2804 & -0.96 & 450 & 354 & 0.00 & 6.SS & 0.00 & 0.08 \\
\hline Aurges: & 256 & 2500 & 2756 & -1.17 & 450 & 3.33 & 0.00 & 6.49 & 0.00 & 0.05 \\
\hline seopmober & 2,45 & 2500 & 27A5 & .125 & 450 & 325 & 0.00 & 6.91 & 0.00 & $O A B$ \\
\hline octobs & 0.09 & 2500 & 2560 & -1.18 & 450 & 332 & 0.00 & $6 A 9$ & 0.00 & 0.00 \\
\hline Noverbox & -1.98 & 2500 & 23.02 & 328 & 450 & 122 & 0.00 & 490 & -0.12 & 0.00 \\
\hline Decerribar & -440 & 25,00 & 20.60 & 4.75 & 450 & -0.25 & -0.05 & 3.58 & -2.16 & 0.00 \\
\hline
\end{tabular}

\begin{tabular}{|c|c|}
\hline Themal & Tot Racm: \\
\hline WWhAvilab & KWhHoating \\
\hline 1204 & (2.65) \\
\hline 1606 & (1.80) \\
\hline 26.76 & $(1.50)$ \\
\hline 31.78 & $(0.14)$ \\
\hline 36.12 & 0.00 \\
\hline 3691 & 0.00 \\
\hline $35 \pm 3$ & 0.00 \\
\hline 3052 & 0.00 \\
\hline 2616 & 0.00 \\
\hline 20.85 & 0.00 \\
\hline 1529 & $(0.12)$ \\
\hline 1121 & \\
\hline
\end{tabular}

CLOWOY DAY HEATBALAYCE

\begin{tabular}{|c|c|c|c|c|c|c|c|c|c|c|}
\hline \multirow{2}{*}{ Month } & \multicolumn{3}{|c|}{ Doaroied Period Wh/h2 } & \multicolumn{3}{|c|}{ Unocospied Period W/m2 } & \multicolumn{2}{|c|}{ Monthy RegiromontkYh } & \multicolumn{2}{|c|}{ FicshifloadkHh } \\
\hline & PVnotgin & Lntornels & Onocel & PVnologin & Intonals & anas & Hoaing & $\operatorname{cosing}$ & Heaing & $\operatorname{cosing}$ \\
\hline Januey & -563 & 4500 & 3937 & 530 & $\overline{450}$ & -0.80 & -0.18 & 6.14 & -252 & 0.00 \\
\hline Fodruary & -450 & 40.00 & 3550 & -144 & 450 & 0.05 & 0.00 & 4.08 & -129 & 0.00 \\
\hline Narch & 327 & 3500 & 31.73 & 359 & 450 & 0.91 & 0.00 & 327 & -0.71 & 0.00 \\
\hline Apri & -211 & 30.00 & 2789 & .255 & 450 & 1.95 & 0.00 & 266 & -0.06 & 0.00 \\
\hline May & -0.58 & 2500 & 24.02 & -1.46 & 450 & 304 & 0.00 & 254 & 0.00 & 0.00 \\
\hline fino & 0.16 & 2500 & 25.16 & -0.36 & 450 & 4.14 & 0.00 & $2 A 1$ & 0,0 & 0.00 \\
\hline Whity & 0.81 & 2500 & 2581 & 0.00 & 450 & 450 & 0.00 & 328 & 0.00 & 0.04 \\
\hline Augest & 0.57 & 2500 & 25.57 & -0.06 & 450 & $4 A$ & 000 & 3.45 & 0.00 & 0.03 \\
\hline Soptrmber & 0.83 & 30.00 & $30: 33$ & 0.06 & 450 & 456 & 0.00 & 3.13 & 0.00 & 0.18 \\
\hline octabor & -0.56 & 35.00 & 3444 & -120 & 450 & 321 & 0.00 & 3.74 & 0.00 & 0.00 \\
\hline Norember & -291 & 40.00 & 37.09 & 328 & 450 & 122 & 0.00 & 395 & -0.06 & 0.00 \\
\hline Decomber & -5.15 & $\mathbf{4 5 0 0}$ & 39.85 & 4.7 & 450 & -027 & -0.05 & $5 \mathrm{MA}$ & -1.69 & 0.00 \\
\hline
\end{tabular}

OVEAALI ENERGYREQURRETENTS AND PRODJCTON

\begin{tabular}{|c|c|c|c|c|c|c|c|}
\hline \multirow[t]{2}{*}{ Manth } & \multicolumn{2}{|c|}{ Coding Consumpion } & \multicolumn{2}{|c|}{ Heating Consermption } & \multirow{2}{*}{$\begin{array}{l}\text { Fans } \\
\text { kith }\end{array}$} & \multirow{2}{*}{$\begin{array}{l}\text { Lighing and } \\
\text { Power kW/h }\end{array}$} & \multirow{2}{*}{$\begin{array}{l}\text { Total Xth } \\
\text { Consumed }\end{array}$} \\
\hline & $1 / 000$ & HermEectic & $1 / 00 p$ & kwh Eectic & & & \\
\hline Januay & 0.00 & 0.00 & 022 & 1.19 & 2.08 & 5.76 & 9.03 \\
\hline Februery & 0.00 & 0.00 & 0.22 & 0.70 & 1.88 & 4.10 & 6.68 \\
\hline Warch & 0.00 & 0.00 & 022 & 0.49 & 208 & 3.63 & 620 \\
\hline Apri & 020 & 249 & 0.22 & 0.04 & 2.01 & 299 & 753 \\
\hline May & 0.29 & 2.61 & 022 & 0.00 & 208 & 2.64 & 733 \\
\hline June & 022 & 2.72 & 0.22 & 0.00 & 201 & 255 & 728 \\
\hline Waty & 029 & 2.93 & 022 & 0.00 & 2.08 & 264 & 7.65 \\
\hline Angus: & 020 & 2.86 & 022 & 0.00 & 208 & 2.64 & 758 \\
\hline Septombor & 029 & 3.06 & 022 & 0.00 & 2.01 & 297 & 8.04 \\
\hline Odtober & 029 & 2.92 & 022 & 0.00 & 2.08 & 3.60 & 8.60 \\
\hline November & 029 & 253 & 022 & 0.04 & 201\} & 4.08 & 8.66 \\
\hline Decomber & 0.00 & 0.00 & 0.22 & 0.88 & 208) & 536 & 8.33 \\
\hline TOTAL/m2 & & 2212 & & 3.34 & 24.51 & 4293 & 9290 \\
\hline
\end{tabular}

Thermal Total Regment kWhAvitabe fkWh Heating

\begin{tabular}{rr|r|}
\hline 2.91 & $(200)$ \\
4.47 & $(1.29)$ \\
5.82 & $(0.71)$ \\
5.39 & $(0.05)$ \\
6.88 & 0.00 \\
7.34 & 0.00 \\
10.16 & 0.00 \\
0.00 & 0.00 \\
5.18 & 0.00 \\
0.00 & 0.00 \\
0.07 & $(0.05)$ \\
4.77 & $(1.75)$ \\
\hline
\end{tabular}

\begin{tabular}{|r|r|}
\hline Thamal Avaz & Tobj kWh \\
\hline kwh Bectic & Consuniod \\
\hline 3.29 & 7.84 \\
4.51 & 5.98 \\
7.19 & 5.71 \\
8.18 & 7.49 \\
9.46 & 7.33 \\
9.73 & 728 \\
10.07 & 7.65 \\
6.72 & 7.58 \\
6.96 & 8.04 \\
4.61 & 8.60 \\
3.38 & 8.62 \\
3.52 & 7.4 \\
\hline 77.62 & 69.56 \\
\hline
\end{tabular}


OPTION 5: PV ROOF HONTORS WITH ACTVE HEAT RECOVERY

\begin{tabular}{|c|c|c|c|c|c|c|c|c|c|c|c|c|}
\hline \multirow{3}{*}{\begin{tabular}{|l|} 
Cty \\
Cimate \\
WEATHERD \\
Manth \\
\end{tabular}} & \multicolumn{2}{|c|}{$\begin{array}{l}\text { Now Yak } \\
\text { Tomporab costal oold }\end{array}$} & \multirow[t]{2}{*}{$\begin{array}{l}\text { Longude } \\
\text { Laivido } \\
\text { Alitudo }\end{array}$} & $\begin{array}{r}40.701 \\
74021 \\
3.00\end{array}$ & & $\begin{array}{l}\text { Dreat Radak } \\
\text { Dithuso Rada }\end{array}$ & $\begin{array}{l}\text { ion Factor } \\
\text { ifon Factor }\end{array}$ & $\begin{array}{l}0.50 \\
1.10\end{array}$ & & & & \\
\hline & & & & & & & & & & & & \\
\hline & 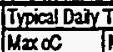 & Tenporatros & 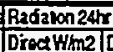 & raveragos & Hars of Suns & sting & Eqivabant ba & ys & Average Her & inty & & \\
\hline anuery & Max 0 & Mnoc & & Dinuso Himn & Total hours & & Sumy & Coucty & Day & Notht & & \\
\hline ebruery & 430 & -2000 & 8500 & 40.00 & 151.00 & 10.00 & 1510 & 15.90 & 61 & 69 & & \\
\hline Fescenty & $\begin{array}{l}4.10 \\
8.90\end{array}$ & $\begin{array}{l}-240 \\
0.50\end{array}$ & 13000 & 5500 & 164.00 & $\begin{array}{c}1028 \\
1183\end{array}$ & $\begin{array}{l}15.96 \\
17.57\end{array}$ & 1204 & 58 & 67) & & \\
\hline Apri & 14.90 & 6.10 & 250.00 & 10000 & 214.00 & 1209 & 17.30 & 作 1230 & $\begin{array}{l}56 \\
55\end{array}$ & 8 & & \\
\hline May & 2090 & 11.80 & 290.00 & 120,00 & 250.00 & 134 & 1860 & 1240 & 56 & 6 & & \\
\hline Jino & 25.60 & 1690 & 305.00 & 125.00 & 301.00 & 16.18 & 18.60 & 1140 & 58 & $\pi$ & & \\
\hline Juty & 2230 & 1990 & 200.00 & 120.00 & 307.00 & 15.00 & 20.46 & 1054 & 57 & 74 & & \\
\hline Avarst & 2740 & 1930 & 250.00 & 100.00 & 275.00 & 1386 & 19.84 & 11.16 & 60 & 76 & & \\
\hline Soplembor & 2380 & 1580 & 190.00 & 75.00 & 277.00 & 1254 & 1890 & 11.10 & 60 & 76 & & \\
\hline Detober & 1840 & 1030 & 130.00 & 5500 & 218.00 & 1134 & 1922 & 11.78 & 59 & 74 & & \\
\hline November & 11.90 & 440 & 85.00 & 40.00 & 17200 & 10.06 & 17.10 & 1290 & 60 & 71 & & \\
\hline Docosmber & 5.70 & -120 & 70.00 & 35.00 & $158.00]$ & 10.00 & 1580 & 15200 & 60 & 67) & & \\
\hline BULFHAL & ATA & & & & PVPANED D & DATA & & & HLAOTIFH & Hive & & \\
\hline $\begin{array}{l}\text { Area of PVP } \\
\text { Area offort } \\
\text { Heat Gain } \\
\text { HealGain th }\end{array}$ & $\begin{array}{l}\text { and per m2 of } \\
\text { a Inght per me of } \\
\text { on Liphts W/m2 } \\
\text { om Eqipment }\end{array}$ & $\begin{array}{l}\sqrt{1600 x} \\
\text { of } 100 x \\
12 \\
\text { iW/m2 }\end{array}$ & $\begin{array}{r}1.00 \\
0.15 \\
20.00 \\
5.00\end{array}$ & & $\begin{array}{l}\text { Converson En } \\
\text { Diroct Trensm } \\
\text { Thermal Trans } \\
\text { PV slope ( }\end{array}$ & $\begin{array}{l}\text { Efficioncy } \\
\text { mitanco } \\
\text { nesritancos Wh } \\
\text { ) }\end{array}$ & $\begin{array}{c}\% \\
\% \\
m\end{array}$ & \begin{tabular}{r|r|}
10.00 & \\
0.00 & \\
020 & \\
1500 & \\
\end{tabular} & $\begin{array}{l}\text { Enth } \\
\text { Entrapy }\end{array}$ & $\begin{array}{r}3 \\
36,41\end{array}$ & & \\
\hline Heat Gain f & om Pooplo W/m & & 20.00 & & 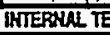 & EXPEERATURI & & & HLYISTYS & 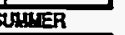 & & \\
\hline $\begin{array}{l}\text { ocoupancy } \\
\text { Docopancy }\end{array}$ & FishTme & & $\begin{array}{r}9.00 \\
19.00\end{array}$ & & $\begin{array}{l}\text { Ocoupiod Perix } \\
\text { Unocapiod P }\end{array}$ & $\begin{array}{l}\text { riod SetPoint } \\
\text { Pariod Orsol }\end{array}$ & $\begin{array}{l}\alpha \\
\alpha\end{array}$ & $\begin{array}{r}21.00 \\
5.00\end{array}$ & $\begin{array}{l}\text { Yor } \\
\text { Entraby }\end{array}$ & $\begin{array}{r}60 \\
47.425\end{array}$ & & \\
\hline CALCULAT & DOHS OF MEAK & NEXTEPMAL & COHDITIOHS & & & & & & & & & \\
\hline Manth & Temporatroc & $\mathscr{C}$ & Sumy Day Ras & 20abon Wmate & Choudy Day $\mathrm{P}$ & gadabon WhI & Egrrabert $\mathrm{Da}_{2}$ & $y$ & Extentraloy & $\mathrm{k} / \mathrm{kg}$ & & \\
\hline & Oospiod II & Unocapiod & ocosoind It & Unocapied & Ocoupied II & Uhocosion & somy & Coundy & Docapiod & Unocapiod & & \\
\hline Januery & 253 & -0.52 & 207.60 & 0.00 & 86.00 & 0.00 & 15.10 & 1500 & 8.95 & 4.83 & & \\
\hline Fabruary & $2 / 8$ & -031 & 293.12 & $5 . \pi$ & 12846 & 253 & 15.96 & 2204 & 856 & 499 & & \\
\hline March & 6.83 & 327 & 360.16 & 4703 & 15218 & 1987 & 17.67 & 1333 & 15.67 & 10.86 & & \\
\hline Apri & 1270 & 8.93 & 46649 & 69.65 & 198.50 & 29.64 & 17.70 & 1230 & 26.71 & 21.80 & & \\
\hline Nity & 18.68 & 14.78 & 494.61 & 12156 & 21427 & 5266 & 18.60 & 1240 & 3998 & 3506 & & \\
\hline Jine & 2343 & 19.70 & 430.09 & 18984 & 18538 & 81.87 & 18.60 & 1140 & $\mathbf{5 2 7 4}$ & 49.03 & & \\
\hline 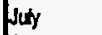 & 2820 & 2260 & 43.06 & 158.39 & 191.24 & 6862 & 20,45 & 10.54 & 59.60 & 5839 & & \\
\hline Acosest & 2538 & 21,20 & 40690 & $11221]$ & 173.15 & 47.75 & 19.84 & 18.16 & 59.05 & 57.48 & & \\
\hline Sepiomber & 2180 & 1837 & 309.72 & 61.53 & 14354 & 26.04 & 1890 & 11.10 & 49.48 & 46.90 & & \\
\hline Decober & 1638 & 1200 & 265.55 & 25.46 & 11638 & 11.16 & 1922 & 11.78 & 3584 & 3204 & & \\
\hline Noventber & 10.03 & 681 & 20639 & 0.86 & 9544 & 0.40 & 17.10 & 1290 & 2245 & 18.01 & & \\
\hline Docomber & 3.98 & 1.02 & 176.40 & 0.00 & 84.00 & .0 .00 & 15.60 & 1520 & 1126 & 7.09 & & \\
\hline SGRWYDA & TEEATQALAN & $\overline{N C E}$ & & & & & & & & & & \\
\hline Month & Oospiod Pai & nodW/m2 & & Unocaspiod Po & exiod W/m2 & & Monthy Rogexi & Fementkthn F & FroshAílos & $2 d x+h$ & Themal & Toex Reoms \\
\hline & PVrotgain & insmals & Overd & PVrotgain I 1 & Intemads & apord & Hoaing & $\begin{array}{ll}\text { Cooling } & \text { I } \\
\end{array}$ & Heaing & Cooking & Whan Awabdi & 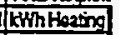 \\
\hline Januery & -1026 & 25.00 & 14.74 & $\cdot 10.74$ & 450 & -624 & $-1,32$ & 222 & -7.96 & 0.00 & 1209 & (923) \\
\hline Fobreary & .958 & 2500 & 1512 & -10.55 & 450 & -6.05 & -1.35 & 246 & -854 & 0.00 & 17.78 & (2.8) \\
\hline March & -6.18 & 25.00 & 1881 & -788 & 450 & 3.38 & -0.84 & 332 & -704 & 0.00 & 2384 & $(7.87)$ \\
\hline Aprit & $-1 A B$ & 25.00 & 2352 & 4.01 & 450 & 0.48 & 0.00 & 123 & -330 & 0.00 & 3053 & (330) \\
\hline Miar & 2.61 & 25.00 & 27.61 & 305 & 150| & $\mathbf{M \mu}$ & 0.00 & 551 & 0.00 & 0.00 & 33.75 & 0.00 \\
\hline Jino & 5.19 & 25.00 & 30.18 & $-1 / 42$ & 450 & 3.08 & 0.00 & 6.42 & 0.00 & 1.90 & 29.16 & 0.00 \\
\hline Juty & 7.10 & 25.00 & 3210 & -0.88 & 450 & 3.62 & 0.00 & 7.61 & 0.00 & 4.78 & 3202 & 0.00 \\
\hline Augest & 626 & 2500 & 3126 & -0.54 & 450| & 3.86 & 0.00 & 728 & 0.00 & 4,43 & 2934 & 0.00 \\
\hline Septuntoser & 3.37 & 25.00 & 2837 & $-1,19$ & 450 & 3.31 & 0.00 & 624 & 0.00 & 0.75 & 2345 & 0.00 \\
\hline loctober & -0.78 & 25.00 & 2422 & -180 & 450 & 270 & 0.00 & 538 & -021 & 0.00 & 1897 & (021) \\
\hline Novembor & -5.40 & 25.00 & 19.60 & -597 & 450| & -147 & -0.35 & 3.35 & -4.58 & 0.00 & 1333 & (4.SA) \\
\hline Docomber & -8.58 & 25.00 & 15.12 & $-9.74 \mid$ & 450] & -524 & -1.16 - & 244 & -7.63 & 0.00 & 10.80 & (879) \\
\hline CLOCOYDA & YYEAT BALA & AHCE & & & & & & & & & & \\
\hline Month & Doapiod Paxx & nod Wm2 & & Unocarpiod Pe & criod W/m2 & & Monfry Rea & Frmentkthif & Fresch Ar Las & $\mathrm{ddkWh}$ & Themal & $\overline{\mathrm{comn}}$ \\
\hline & PY notgain I & Intemals & Owoenl & PVnol gain It & Internals & Toverd! & Heaing & & & Tcooling & Whaveda d & ating \\
\hline$\sqrt{\text { andery }}$ & -1120 & 45.00 & 3380 & -10.74 & 450 & $-624 \mid$ & $-1,39$ & 537 & -238 & 0.00 & 4,48 & (9.7n) \\
\hline Fobruary & -10.96 & 40.00 & 20.04 & -10.58 & 4.50 & -6.08 & -102 & 350 & $-6,4$ & 0.00 & 539 & (7.45) \\
\hline March & -7.94 & 35.00 & 27.06 & \&.19] & 450 & -3.61 & -0.67 & 3.61 & -531 & 0.00 & 657 & $(5.80)$ \\
\hline Apri & -3.73 & 30.00 & 2627 & 435 & 450 & 0.15 & 0.00 & 326 & -228 & 0.00 & 827 & (220) \\
\hline May & 026 & 25.00 & 2528 & -0.30 & 450 & 4.11 & 0.00 & 385 & 0.00 & 0.00 & 930 & 0.00 \\
\hline Jino & 3.13 & 2500 & 28.13 & -0.48 ; & 450 & 4.02 & 0.00 & 385 & 0.00 & 1.16 & 8.58 & 0.00 \\
\hline Juty & 4.99 & 25.00 & 29.99 & -0.01 & 450 & 449 & 0.00 & 382 & 0.00 & 245 & 1049 & 0.00 \\
\hline Augus: & 4.30 & 25.00 & 2930 & $-\infty .09$ & 450 & 4A1 & 0.00 & 395 & 0.00 & 249 & 0.00 & 0.00 \\
\hline Soptember & 1.73 & 30.00 & 31.73 & -1.16 & 450 & 3.34 & 0.00 & 4.04 & 0.00 & 0.44 & 523 & 0.00 \\
\hline Dations & -203 & 3500 & 3297 & -1.92 & 450 & 258] & 0.00 & 431 & -0.13 & 0.00 & 0.00 & $(0.13)$ \\
\hline Novembor & -633 & 40.00 & 33.67 & -5.97 & 450 & $-1,47$ & -0.27 & 434 & 3,45 & 0.00 & 0.07 & (3.72) \\
\hline Docomber & -1036 & 45.00 & 34.64 & -9.74 ] & 450] & .524 & -1.11 & 527] & -7.34 & 0.00 & 5.18 & $(8,45)$ \\
\hline OVERALLE & NERGYREQUA & RREMENTSN & WDPRODUCT & तOH & & & & & & & & \\
\hline Menth & Cooing Const & Impion & Heaing Cons & unotion & Fans & Lighting and & 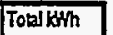 & & & & Thermal Avat: & \\
\hline & 11000 & knmEectic & $11 \log$ & Whe Hectic & Wh & Powor Kth & Consemod & & & & then Bectic & Consemed \\
\hline$\sqrt{\text { tancary }}$ & 0.00 & 0.00 & 022 & 423 & 230 & 5.82 & 1235 & & & & 3.65 & 8.11 \\
\hline Fobruary & 0.00 & 0.00 & 0.2 & 386 & 208 & 4.19 & 80.12 & & & & 5.10 & 626 \\
\hline March & 0.00 & 0.00 & 022 & 3.08 & 230 & 3.97 & 9.35 & & & & 6.59 & 627) \\
\hline Apri & 0.00 & 0.00 & 022 & 124 & 223 & 3.17 & 6.63 & & & & 854 & 5.39 \\
\hline May & 0.29 & 257 & 022 & 0.00 & 230 & 264 & 7.61 & & & & 8,27 & 7.61 \\
\hline Jing & 028 & 3.81 & 022 & 0.00 & 223 & 255 & 858 & & & & 832 & 858 \\
\hline Juty & 028 & 5.34 & 022 . & 0.00 & 230 & 2.54 & 10227 & & & & 9.55 & 1027 \\
\hline Aegust & 029 & 5.19 & 0.22 & 0.00 & 230 & 2.64 & 10.12 & & & & 6A5 & 10.12 \\
\hline Soplomber & 029 & 327 & 022 & 0.00 & 223 & 3.11 & 8.61 & & & & 6.31 & 8.61 \\
\hline loctobor & 029 & $2 \pi$ & 022 & 0.08 & 230 & 381 & 8.96 & & & & 4.15 & 8.88 \\
\hline November & 0.00 & 0.00 & 02 & 1.22 & 223 & 4.48 & 8.8 & & & & 205 & 6.71 \\
\hline Decomber & 0.00 & 0.00 & 022 & 3.83 & 230 & 5.68 & 11.81 & & & & 352 & 7.97 \\
\hline TOTNLm2 & & 2305 & & 1825 & 27.07 & 44.67 & 113.04 & & & & 74.70 & 94.78 \\
\hline
\end{tabular}


OPTION 5: PV ROOF MONTORS WITH ACTVE HEAT RECOVERY

\begin{tabular}{|c|c|c|c|c|c|c|c|c|c|c|c|c|}
\hline$c^{a t y}$ & $\begin{array}{l}\text { Phoordx } \\
\text { Temparato ais }\end{array}$ & & $\begin{array}{l}\text { Longitude } \\
\text { Laitude } \\
\text { Alitude }\end{array}$ & $\begin{array}{r}33.43 \\
112.02 \\
340.00\end{array}$ & & $\begin{array}{l}\text { Droct Padaí } \\
\text { Dillose Radus }\end{array}$ & $\begin{array}{l}\text { ion Factor } \\
\text { lion Factox }\end{array}$ & $\begin{array}{l}1.10 \\
0.90\end{array}$ & & & & \\
\hline WEATEER & ATA & & & & & & & & & & & \\
\hline Marth & Typical Dajy & Terrocatres & Radaion 241 & raverages & Hotrs ol Suns & $\sin \theta$ & Equialont Da & & Averaog Hum & ifify $\%$ & & \\
\hline & $M a x \infty$ & $\sin \alpha$ & Droct W/m2 & Diffuso $\mathrm{Wman}$ & Total hours & Maxporday & Sumy & Coundy & Day & Nognt & & \\
\hline Janery & 1780 & 180 & 105.00 & 2500 & 26200 & 1084 & 24.18 & 682 & 44 & 72 & & \\
\hline Fabruery & 20.10 & 3800 & 150.00 & 30.00 & 271.00 & 1241 & 2184 & 6.16 & 42 & 71 & & \\
\hline Merch & 2390 & 6.10 & 20500 & $35 \infty \infty$ & 351.00 & 1256 & 26.35 & 4.65 & 31 & 60 & & \\
\hline Appi & 2880 & 1020 & 250.00 & 45.00 & 360.00 & 1348 & 26.70 & 330 & 26 & 50 & & \\
\hline$n \in y$ & 33800 & 1390 & 25500 & 45.00 & 408.00 & 14.00 & 29.14 & 186 & 19 & 41 & & \\
\hline timo & 3970 & 1860 & 30500 & 50.00 & 411.00 & 1457 & 2820 & 100 & 17] & 37 & & \\
\hline buty & 40.30 & 2390 & 255.00 & 4500 & 300.00 & 1430 & 2728 & 3.72 & 29 & 51 & & \\
\hline Auges & 38.70 & 2300 & 260.00 & 4500 & 379.00 & 142 & 26.66 & 434 & 35 & 60 & & \\
\hline Septomber & 3680 & 19.60 & 205.00 & 3500 & 354.00 & 13.11 & 27.00 & 3.00 & 29 & 66 & & \\
\hline Oetcoer & 30.40 & 1260 & 150.00 & 30.00 & 323.00 & 11.98 & 26.97| & 4.03 & 30 & 58 & & \\
\hline Norenbex & 2320 & 580 & 105.00 & 2500 & 28200 & 10.90 & 2580 & 420 & 33 & $83)$ & & \\
\hline Decentiber & 1890 & 280 & 90.00 & 25.00 & 260.00 & 10.75 & 24.18 & 682 & 42 & 70 & & \\
\hline ELDAHG & ATA & & & & PVPARE D & ATA & & & 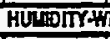 & SNER & & \\
\hline $\begin{array}{l}\text { Area of PV } \\
\text { Area of Nort } \\
\text { Heat Gain th } \\
\text { Heat Gain th }\end{array}$ & $\begin{array}{l}\text { and per m2 of } \\
\text { ight per me of } \\
\text { on Lights W/m2 } \\
\text { on Eatpment }\end{array}$ & $\begin{array}{l}\text { Lox } \\
\text { ttoox } \\
2 \\
W / \pi 2\end{array}$ & $\begin{array}{r}1.00 \\
0.15 \\
20.00 \\
5.00\end{array}$ & & $\begin{array}{l}\text { Corwersion E } \\
\text { Oiroct Transis } \\
\text { Thermal Tran } \\
\text { PV stope () }\end{array}$ & $\begin{array}{l}\text { Dixiency } \\
\text { vittancos } \\
\text { nsmitancos Wh }\end{array}$ & $\begin{array}{c}\% \\
\% \\
m 20 c^{2}\end{array}$ & \begin{tabular}{r|}
10.00 \\
0.00 \\
0.20 \\
1500
\end{tabular} & $\begin{array}{l}\text { FoH } \\
\text { Entralyy }\end{array}$ & \begin{tabular}{r|}
35 \\
35.41 \\
\end{tabular} & & \\
\hline Heat Gaint & on Pooplo W/h & & 2000 & & INIERIL T & ETPERATRE & & & HITIDIT-SU & UITER & & \\
\hline $\begin{array}{l}\text { Ocoupancy } \\
\text { Ocapancy }\end{array}$ & $\begin{array}{l}\text { Shat Tme } \\
\text { nish Trme }\end{array}$ & & $\begin{array}{r}9.00 \\
18.00\end{array}$ & & $\begin{array}{l}\text { Dospied Por } \\
\text { Unocapiod P }\end{array}$ & $\begin{array}{l}\text { riod SolPoint } \\
\text { Period Olsol }\end{array}$ & $\begin{array}{l}\infty \\
\alpha\end{array}$ & $\begin{array}{r}21.00 \\
5.00\end{array}$ & $\begin{array}{l}\text { FoH } \\
\text { Entraby }\end{array}$ & $\begin{array}{r}60 \\
47 \text { A2S }\end{array}$ & & \\
\hline CALCULAT & IOHS OF UEA & NEXTERNAL & CONDIIRONS & & & & & & & & & \\
\hline Month & Tenperairoo & & Sum Day Ra & asision W/m2 & Cloudy Day R & RadabonWmal & Equialont Da & & ExtEntralpy & $k \sqrt[k]{k g}$ & & \\
\hline & Cospiod & thoocipiod & Docoiniod I & throcaniod & Oocoiod & Thocapiod & Suroy & Coosty & oosoind & Uhoospiod & & \\
\hline Januay & 1380 & 694 & 305.66 & 1824 & 110.75 & 6.61 & 24.18 & 682 & 2594 & 1843 & & \\
\hline Fobruay & 1608 & 8.04 & 37.36 & 0389 & 116.05 & 1996 & $2 t .84$ & 6.16 & 2956 & 2260 & & \\
\hline March & 19,45 & 11.82 & 491.02 & 8984 & 133.74 & 24,47 & 2635 & 4.65 & 3188 & 26.13 & & \\
\hline Api & 24.15 & 16.18 & 581.17 & 144.59 & 16020 & 39.66 & 26.70 & 330 & 3782 & 3246 & & \\
\hline Nay & 2883 & 2030 & 625.65 & 178.82 & 15427 & 4.09 & 28.14 & 188 & 41.52 & 37.62 & & \\
\hline Jun & 33.68 & 2506 & 62658 & 204.73 & 164.67 & 5381 & 2820 & 180 & 4787 & 4549 & & \\
\hline forty & 3520 & 29.17 & 61275 & 188.04 & 151.09 & 4636) & 2728 & 3.72 & 63.15] & 6382 & & \\
\hline August & 34.78 & 2805 & 55121 & 165.99 & 151.24 & 45.75 & 26.66 & 434 & 6511 & $\infty \ldots$ & & \\
\hline Septantiber & 3250 & 25.13 & $470 \mathrm{AP}$ & 10454 & 128.14 & 2847 & 27.00 & 3.00 & 55.49 & 61.70 & & \\
\hline octabex & 25.95 & 1832 & 384.76 & 5431 & 12024 & 1697 & 26.97 & 4.03 & 4331 & 40.03 & & \\
\hline November & 18.85 & 1139 & 303.01 & 20.13 & 109.79 & 729 & 2580 & 420 & $3 t .61$ & 30.54 & & \\
\hline Decerriber & 14.88 & 798 & 271.19 & 1458 & 111.60 & 6.00 & 24.18 & 682 & 2738 & 2020 & & \\
\hline SLAWYDA & THEAT BALAN & & & & & & & & & & & \\
\hline Manth & Oocopiod Pei & iod W/m2 & & UnocoupiodP & Period W/m2 & & Montsy Regi & iromentkwh & FreshAirloar & dkwh & Thermat & Tow Foomt \\
\hline & PVnotgain & Intmels & Ovoral & PVnetgain & Thromads & Toveral & Hoaing & Cooing & Hoaing & Cooing & kTh' Avaibde & knh Hoating \\
\hline Januery & -211 & 2500 & 2289 & -5.73 & 450 & -123 & -0.42 & 5.53 & 486 & 0.00 & 27.13 & (520) \\
\hline Fidruey & -0.11 & 25.00 & 2489 & -399 & 450 & 051 & 0.00 & 559 & -288 & 0.00 & 29.91 & (288) \\
\hline March & 3.12 & 2500 & 2812 & -1.96 & 450 & 254 & 0.00 & 8.35 & -220 & 0.00 & $47 / 2$ & (220) \\
\hline Apri & 6.93 & 2500 & 31.93 & -1.22 & 450 & 258 & 0.00 & 8.40 & 0.00 & 0.00 & 5657 & 000 \\
\hline Mag & 1034 & 2500 & 3534 & 1.04 & 4.50 & 554 & 0.00 & 1256 & 0.00 & 0.00 & 66.18 & 0.00 \\
\hline & 1350 & 25.00 & 3850 & 1.11 & 450 & 561 & 0.00 & \{3.07 & 0.00 & 024 & 6283 & 0.00 \\
\hline Juty & 15.03 & 2500 & 40.03 & 3.64 & 450 & 8.14 & 0.00 & 14.03 & 0.00 & 824 & 6021 & 0.00 \\
\hline August & 1358 & 2500 & 3858 & 2.72 & 450 & 722 & 0.00 & 1298 & 0.00 & 921 & 5293 & 0.00 \\
\hline Septenber & 11,43 & 25.00 & 3643 & 0.31 & 450 & 4.81 & 0.00 & 11.65 & 0.00 & 4.18 & 45.75 & 0,00 \\
\hline octcober & 6.45 & 2500 & 31.45 & -128 & 450 & 322 & 0.00 & 9.70 & 0.00 & 0.00 & 37.65 & 0.00 \\
\hline Novernioer & 1.15 & 25.00 & 26.15 & -283 & 450 & 1.57 & 0.00 & 735 & -238 & 0.00 & 2872 & (239) \\
\hline Docomber & -1.70 & 25.00 & 2330 & -509 & 450 & -0.50 & -020 & 5.60 & 4.49 & 0.00 & 2432 & (4.39) \\
\hline CLOUOYD & AY KEAT BALA & AKCE & & & & & & & & & & \\
\hline Mants & Oocapiad Pen & $00 \mathrm{~W} / \mathrm{m} 2$ & & UnocapiodP & PeriodW/m2 & & Monthy Paqu & Irementkwh & Frosh Air Loa & doknh & Thermal & Tool Reams \\
\hline & Py nelgain & Inomels & Owerl & PV notgain & insmals & Overa! & Hoaing & $\cos 50$ & Hoging & Cooting & xwh Apoibdid & okthh Hoating \\
\hline flaxtary & 3.75 & 45.00 & 4125 & -583 & 450 & -133 & -0.13 & 281 & -137 & 0.00 & 0.76 & (1.50) \\
\hline Fabruary & -226 & 40.00 & 37.74 & 436 & 450 & 0.14 & 0.00 & 234 & -0.81 & 0.00 & 1.22 & $(0.81)$ \\
\hline March & 0.12 & 35.00 & 35.12 & $-25 t$ & 450 & 1999 & 0.00 & 1.76 & 0.40 & 0.00 & 2.14 & $(0.40)$ \\
\hline & 339 & 30.00 & 3339 & -1.17 & 450 & 333 & 0.00 & 126 & 0.00 & 0.00 & 1.74 & 0.00 \\
\hline Hey & 6.38 & 25.00 & 34.38 & -0.09 & 450 & $4 A 1$ & 0.00 & 0.70 & 0.00 & 0.00 & 220 & 0.00 \\
\hline Juno & 9.62 & 2500 & 34.52 & 1.47 & 4.50 & 5.97 & 0.00 & 0.77 & 0.00 & 0.02 & 211 & 0.00 \\
\hline futy & 11.15 & 25.00 & 36.15 & 245 & 450 & 6.55 & 0.00 & 1.71 & 0.00 & 9.12 & 354 & 0.00 \\
\hline August & 1023 & 2500 & 3523 & 1.71 & 450 & 621 & 0.00 & 191 & 0.00 & 1.50 & 0.00 & 0.00 \\
\hline Septenter & 855 & 30.00 & 3855 & -0.33 & 450 & 4.17 & 0.00 & 133 & 0.00 & 0.46 & 4.43 & 0.00 \\
\hline Oatconer & 423 & 35.00 & 3923 & - $\quad-1.60$ & 450 & 200 & 0.00 & 1.74 & 0.00 & 0.00 & 0.00 & 0.00 \\
\hline November & -0.48 & 40.00 & 3952 & -293 & 450 & 157 & 0.00 & 1.75 & -030 & 0.00 & 0.08 & $(0.39)$ \\
\hline Docomber & -3.04 & 45.00 & 41.96 & -5.17 & 450 & -0.67 ] & -0.06 & 286 & -1.18 & 0.00 & 6.33 & (1.25) \\
\hline OVERAL & MERGYAEQU & MREKENTSA & AND PROOUC & TON & & & & & & & & \\
\hline Month & Cooing Cons & umpion & Heating Conse & imotion & Fans & Lighting and & Totalkth & & & & ThermalAval & Toblkwh \\
\hline & $\pi / 000$ & kWh Enctic & $1100 p$ & Wh Eectic & knsh & Powor kHh & Consumed & & & & xwh Exatic & Consumed \\
\hline Janxay & 0.00 & 0.00 & 022 & 151 & 293 & 4.00 & 8.44 & & & & 620 & 6.93 \\
\hline February & 0.29 & 227 & 022 & 0.82 & 2.65 & 3.30 & 9.04 & & & & 694 & 822 \\
\hline March & 029 & 289 & 022 & 0.60 & 293 & 3.10 & 9.52 & & & & 10.90 & 892 \\
\hline Apri & 029 & 3.07 & 022 & 0.00 & 2.84 & 2.72 & 8.62 & & & & 1283 & 8.52 \\
\hline May & 0.29 & 3.79 & 022 & 0.00 & 293 & 2.64 & 936 & & & & 1504 & 936 \\
\hline Jumo & 0.29 & 403 & 022 & 0.00 & 2.84 & 255 & 9.42 & & & & 1457 & $9 / 12$ \\
\hline fus & 029 & 7.17 & 022 & 0.00 & 293 & 2.64 & 1274 & & & & 1402 & 1274 \\
\hline Aughes & 0.29 & 739 & 022 & 0.00 & 293 & 264 & 1268 & & & & 11.64 & 1288 \\
\hline Septrmber & 0.29 & 5.04 & 022 & 0.00 & 2.84 & 2.70 & 10.57 & & & & 11.04 & 1057 \\
\hline Octabor & 0.29 & 327 & 022 & 0.00 & 293 & 3.04 & 924 & & & & 628 & 924 \\
\hline Noventber & 0.29 & 260 & 022 & 0.61 & 284 & 3.18 & 923 & & & & 634 & 8.62 \\
\hline Docember & 0.00 & 0.00 & 022 & 125 & 293 & 4,00 & 8.18 & & & & 6.74 & 6.93 \\
\hline TOTAL/m2 & & 41.43 & & 4.78 & 3453 & 3649 & 11724 & & & & 12456 & 11245 \\
\hline
\end{tabular}


OPTION 5: PY ROOF MONITORS WITH ACTIVE HEAT RECOVERY

\begin{tabular}{|c|c|c|c|c|c|c|c|c|c|c|}
\hline \multirow{2}{*}{$\begin{array}{l}\text { City } \\
\text { Cimato } \\
\text { WEATHERD }\end{array}$} & \multicolumn{2}{|l|}{ Subtropical } & $\begin{array}{l}\text { Longitude } \\
\text { Lailude } \\
\text { Artude }\end{array}$ & \multicolumn{2}{|c|}{$\begin{array}{l}2580 \mathrm{~N} \\
8027 \mathrm{~W} \\
200 \mathrm{~m}\end{array}$} & \multicolumn{2}{|c|}{$\begin{array}{l}\text { Direct Radaion Factr } \\
\text { Dituso Padaion Factr }\end{array}$} & $\begin{array}{l}0.50 \\
1.10\end{array}$ & & \\
\hline & \multicolumn{10}{|c|}{ WEATHERDATA } \\
\hline \multirow[t]{2}{*}{ Mand } & \multicolumn{2}{|c|}{ Iypical Dary Temooralres } & Radaion 24h & $x$ averoos & \multicolumn{2}{|c|}{ Hours of sunstine } & \multicolumn{2}{|c|}{ Equiplont Days } & \multicolumn{2}{|c|}{ Average Humidiy \% } \\
\hline & $\sqrt{M_{2 x} \alpha}$ & MinoC & Drect Whn2 & DimusoWhat & Totalhars II & Maxparday & Surny & Coowdy & Dey & Noth \\
\hline Janxay & 2430 & 1440 & 150.00 & 65.00 & 23900 & 1151 & 20.77 & 1023 & 59 & 84 \\
\hline Fabreny & 25.00 & 1490 & 185.00 & 80.00 & 233.00 & 11.72 & 1988 & 8.12 & 56 & 84 \\
\hline Hech & 26.60 & 1620 & 235.00 & 8500 & 250.00 & 1222 & 201 & 899 & 56 & $\mathscr{2}$ \\
\hline Api & 2810 & 1880 & 275.00 & 11500 & 283.00 & 13.10 & 2150 & 840 & 56 & 79 \\
\hline Mis & 29.70 & 20.20 & 29500 & 12500 & 27600 & 13,4 & 20.46 & 1054 & 50 & 78 \\
\hline thine & 31.10 & $23: 10$ & 285.00 & 12500 & 255.00 & 1393 & 1830 & 11.70 & 64 & 81 \\
\hline fisty & 31.60 & 22.70 & 295.00 & 12500 & 27000 & 1382 & 1953 & $11 \times 7$ & 64) & 81 \\
\hline August & 3210 & 2380 & 275.00 & 11500 & 261.00 & 13.16 & 19.84 & 11.16 & $\mathbf{\infty}$ & 83 \\
\hline Septoribet & 31.10 & 22370 & 235.00 & 8500 & 21200 & 11.88 & 17.70 & 1230 & 66 & \&s \\
\hline Dotcher & 2030 & 21.50 & 185.00 & 80.00 & 200,00 & 11.73 & 17.05 & 1385 & 64 & 85 \\
\hline Noventer & 2680 & 18.10 & 150.00 & 6500 & 22400 & 1131 & 19.80 & 1020 & 60 & 84. \\
\hline Docomber & 26.10 & 15.10 & 135.00 & 5500 & 2100 & 11.14 & 19.84 & 11.16 & 6) & 85 \\
\hline \multicolumn{4}{|c|}{ BU:DOHGDATA } & & \multicolumn{4}{|c|}{ PVPAREIDATA } & \multicolumn{2}{|c|}{ HUTOTYHWTER } \\
\hline \multirow{3}{*}{\multicolumn{3}{|c|}{ 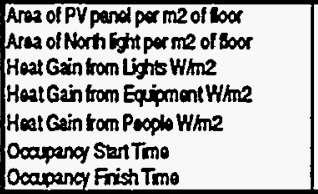 }} & $\begin{array}{r}1.00 \\
0.15 \\
20.00 \\
5.00\end{array}$ & & \multicolumn{3}{|c|}{ 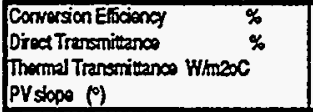 } & $\begin{array}{r}10.00 \\
0.00 \\
0.20 \\
15.00\end{array}$ & FFH & $\begin{array}{r}35 \\
3641\end{array}$ \\
\hline & & & 20.00 & & \multicolumn{4}{|c|}{ WKTERWLL TELPEERATURES } & \multicolumn{2}{|c|}{ HLTSTYSUTISA } \\
\hline & & & $\begin{array}{r}9.00 \\
19.00\end{array}$ & & \multicolumn{3}{|c|}{ 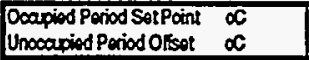 } & $\begin{array}{r}21.00 \\
5.00 \\
\end{array}$ & EFH & $\begin{array}{r}60 \\
47,25\end{array}$ \\
\hline \multicolumn{11}{|c|}{ CMLCULATIOHS OF MEANEXTERMLL COKOITIOHS } \\
\hline \multirow[t]{2}{*}{ Month } & \multicolumn{2}{|c|}{ Tomperalue oC } & Sum Day $F$ & adabonWhind & Coody Day & agibon W/m & Eqrialent De & ars & ExtEntaloyk & $\mathrm{kg} / \mathrm{kg}$ \\
\hline & Ocoupiod & thocaipiod & Dowpiad & Uhroconiod & Oeapiod II & Uhocapied & Survy & Coudy & Oocaiod & Uhocenoind \\
\hline Januery & 21.83 & 1758 & 30555 & 3280 & 13557 & 1459 & 20.7 & 1023 & 49.08 & 4757 \\
\hline Fobruary & 2248 & 18.15 & 379.85 & 46.58 & 16382 & 20.13 & 19.88 & 8.12 & 4932 & 4923 \\
\hline March & 24,00 & 19.54 & 435.25] & 69.18] & 186.55 & 28.60 & 2201 & 8999 & 5321 & 5264 \\
\hline Apri & 25.78 & 21.79 & 483.60 & 107.15 & 210.66 & 46.67 & 21.50 & 840 & 5788 & 5821 \\
\hline May & 2750 & 23.73 & 507.05 & 12639 & 22239 & 55.43 & 20.46 & 1054 & 6443 & 6282 \\
\hline Juno & 29.10 & 25.67 & 490.87| & 137.25 & 21529 & 6050 & 1830 & 11.70 & 72,43 & 7186 \\
\hline July & 29.63 & 2624 & 494.76 & 135.17] & 217.00 & 5929 & 18.53 & $11, A 7$ & 74.06 & 7380 \\
\hline Nergest & 30.03 & 2647 & 481.63 & 108.55 & 209.80 & 4728 & 19.84 & 11.16 & 7460 & 7578 \\
\hline Seplanber & 2925 & 26.08 & 44484 & 6283 & 19036 & 2689 & 17.70 & 1230 & 7426 & 7558 \\
\hline Octubor & 2738 & 24.08 & 37953 & 46.91 & 163.60 & 2023 & 17.05 & 1395 & 67.19 & 6860 \\
\hline November & 24.63 & 2090 & 310.79 & 29.15 & 13789 & 1293 & 1980 & 1020 & 5700 & 57.67 \\
\hline Decortber & 2335 & 18.64 & 275.78 & 2244 & 11850 & 8.64 & 19.84 & 11.16 & 5356 & 51.08 \\
\hline
\end{tabular}

SUWWYOAY EEAT BNLAKCE

\begin{tabular}{|c|c|c|c|c|c|c|c|c|c|c|}
\hline \multirow[t]{2}{*}{ Mondt } & \multicolumn{3}{|c|}{ Oconoiod PeriodW/m2 } & \multicolumn{3}{|c|}{ Unnocuoiod Pariod W/m2 } & \multicolumn{2}{|c|}{ Monthy Requirement thith } & \multicolumn{2}{|c|}{ Fresh Airload kWh } \\
\hline & PVnatgin & Intwrials & Overil & PYnotgin & Tintertals & Ovaral & Heaing & Cooling & Hoging & Cooting \\
\hline Jaxuery & 3.10 & 2500 & 28.10 & -130 & 450 & 320 & 0.00 & $6 . \bar{n}$ & 0.00 & 0. \\
\hline Februery & 4.15 & 2500 & 29.15 & -0.81 & 450 & 3.00 & 0.00 & 682 & 0.00 & .12 \\
\hline March & 5.61 & 25,00 & 30.61 & -0.69 & 450 & 381 & 0.00 & 791 & 0.00 & 245 \\
\hline Apri & 7.17 & 2500 & 3217 & -021 & 450 & 428 & 0.00 & 824 & 0.00 & 434 \\
\hline Hoj & 848 & 25.00 & 33.48 & 0.57 & 450 & 5.17 & 0.00 & 833 & 0.00 & 6.68 \\
\hline $\operatorname{din} \theta$ & 930 & 2500 & $34 \approx 8$ & 0.85 & 450 & 5.45 & 0.00 & 7.69 & 0,00 & 8.76 \\
\hline July & 8.76 & 2500 & 34.76 & 129 & 450 & 5.79 & 0.00 & 8.37 & 0.00 & 9.90 \\
\hline Augast & 991 & 2500 & 34.91 & 122 & 450 & 5.72 & 0.00 & 8.51 & 0.00 & 10.35 \\
\hline Soptombor & 9.10 & 2500 & 34.10 & 0.58 & 450 & 5.08 & 0.0 & 729 & 0.00 & -9.12 \\
\hline & 733 & 2500 & 3233 & 023 & 450 & 4.73 & 0.00 & 6.61 & 0.00 & $6 A 7$ \\
\hline November & 4.97 & 25.00 & 29.97 & $-0,07$ & 450 & 4.03 & 0.00 & 7.05 & 0.00 & 3.6 \\
\hline Desember & 384 & 25,00 & 2884 & -0.70 & 450 & 3800 & 0.00 & 6.78 & 0.00 & 234 \\
\hline
\end{tabular}

CLOLOYDAYHEATBALAHCE

\begin{tabular}{|c|c|c|c|c|c|c|c|c|c|c|}
\hline \multirow[t]{2}{*}{ Month } & \multicolumn{3}{|c|}{ Oospied Period W/m2 } & \multicolumn{3}{|c|}{ Unoospiod Pesiod W/m2 } & \multicolumn{2}{|c|}{ Monty Regiramentk'th } & \multicolumn{2}{|c|}{ Freshirload kWh } \\
\hline & PVnat cein & Intareds & Ouarl & PVnotgin & intamals & Overal & Keaing & Cooing & Hoaling & Cooling \\
\hline Jaxery & 168 & 4500 & 46.68 & .0 .17 & 4.50 & 4.03 & 0.00 & 535 & 0.00 & 0,35 \\
\hline Fabruary & 233 & 40.00 & 4233 & .0 .50 & 150 & 390 & 0.00 & 3.88 & 0.00 & 030 \\
\hline March & 352 & 3500 & 38.52 & -0.37 & 450 & 4.13 & 0.00 & 398 & 0.00 & 1.00 \\
\hline Apri & 487 & 30.00 & 3487 & -0.18 & 450 & 432 & 0.00 & 3.44 & 0.00 & 1.69 \\
\hline Noy & 6.09 & 2500 & 31.09 & 0.07 & 450 & 457 & 0.00 & 395 & 0.00 & 3.44 \\
\hline Jino & 7.07 & 25.00 & 3207 & 029 & 450 & 4.79 & 0.00 & 454 & 0.00 & 5.62 \\
\hline Wil) & 743 & 2500 & 3243 & 0.65 & 450 & 5.15 & 0.00 & 455 & 0.00 & 586 \\
\hline Augest & 7.63 & 25.00 & 3203 & 0.70 & 4.50 & 520 & 0.00 & 4.45 & 0.00 & 582 \\
\hline Sepromber & 6.96 & 30.00 & 36.96 & 028 & 4.50 & 4.78 & 0.00 & 5.37 & 0.00 & 6.34 \\
\hline October & 5.52 & 35.00 & 40.52 & 0.00 & 450 & 450 & 0.00 & 6.53 & 0.00 & 529 \\
\hline Norember & 351 & 40,00 & 4351 & -028 & 450 & 422 & 0.00 & 5.04 & 0.00 & 187 \\
\hline December & 252 & 45,00 & 47.52 & -0.37 & 450 & 4.13 & 0.00 & 5.95 & 0.00 & 1.31 \\
\hline
\end{tabular}

\section{OVERALL ENERGY REQUREUENTS ALD PRODUCTIOH}

\begin{tabular}{|c|c|c|c|c|c|c|c|}
\hline \multirow[t]{2}{*}{ Manth } & \multicolumn{2}{|c|}{ Coding Consempion } & \multicolumn{2}{|c|}{ Heating Consumption } & \multirow{2}{*}{$\begin{array}{l}\text { Fans } \\
\text { twh }\end{array}$} & \multirow{2}{*}{$\begin{array}{l}\text { Lighing and } \\
\text { Power WWh }\end{array}$} & \multirow{2}{*}{$\begin{array}{l}\text { Toal kHh } \\
\text { consermod }\end{array}$} \\
\hline & $1 / \operatorname{cop}$ & xwh Boctic & $1 / 000$ & KKM Electic & & & \\
\hline Januery & 029 & 3.75 & 022 & 0.00 & 2.62 & 4.68 & 11.05 \\
\hline Fobruary & 028 & 335 & 022 & 0.00 & 237 & 3.60 & 931 \\
\hline March & 029 & 4.38 & 022 & 0.00 & 2.62 & 3.53 & 1054 \\
\hline Apri & 029 & 5.06 & 022 & 0.00 & 253 & 297 & 1056 \\
\hline May & 029 & $6 A 0$ & 022 & 0.00 & 262 & 2.54 & 11.66 \\
\hline Jino & 0.29 & 7.61 & 022 & 0.00 & 253 & 255 & 1269 \\
\hline Juty & 0.29 & 822 & 0.22 & 0.00 & 2.62 & 264 & 1347 \\
\hline Algust & 0229 & 833 & 0.22 & 0.00 & 2.62 & 2.64 & 1358 \\
\hline Sepromber & 029 & 8.03 & 022 & 0.00 & 253 & 3.17 & 1378 \\
\hline Ocabor & 029 & 7.12 & 022 & 0.00 & 2.62 & 403 & $137 \pi$ \\
\hline Noventber & 029 & 5.03 & 0.22 & 0.00 & 253 & 4.08 & 11.64 \\
\hline Decanber & 029 & 4.68 & 0.22 & 0.00 & 2.62 & 4.87 & 1217 \\
\hline TOTALm2 & & 71.96 & & 0.00 & 3084 & 41,38 & 144.17 \\
\hline
\end{tabular}

\begin{tabular}{|c|c|}
\hline Thermal & Total Regm \\
\hline 23.18 & 0.00 \\
\hline 2758 & 0.00 \\
\hline 3495 & 000 \\
\hline 37.96 & 0.00 \\
\hline 37.65 & 0.00 \\
\hline 325 & 0.00 \\
\hline 34.97 & 0.00 \\
\hline 34.55 & 0.00 \\
\hline 2847 & 0.00 \\
\hline 2315 & 0,00 \\
\hline 2235 & 0.00 \\
\hline 18.91 & 0.00 \\
\hline
\end{tabular}

\begin{tabular}{|c|c|}
\hline andral & Tot Rocm \\
\hline indavitas & kth Heatix \\
\hline 5.78 & 0.00 \\
\hline 6.84 & 0.00 \\
\hline 754 & 0.00 \\
\hline 9.35 & 0.00 \\
\hline 11.14 & 0.00 \\
\hline 785 & 0.00 \\
\hline 8.64 & 0.00 \\
\hline 0.00 & 0.00 \\
\hline 6.78 & 0.00 \\
\hline 0.00 & 0.00 \\
\hline 0.10 & 0.00 \\
\hline 6.42 & 0.0 \\
\hline
\end{tabular}

\begin{tabular}{|r|r|}
\hline Thermed Avaj & Topl kWh \\
kHh Boctic & Consemed \\
\hline 6.37 & 11.55 \\
7.57 & 9.31 \\
9.35 & 10.54 \\
10.41 & 10.55 \\
10.73 & 11.65 \\
8.58 & 12.69 \\
9.59 & 13.47 \\
7.60 & 1358 \\
7.76 & 1373 \\
5.15 & 13.77 \\
4.94 & 11.64 \\
5.79 & 12.17 \\
\hline 94.15 & 144.17 \\
\hline
\end{tabular}


OPTION 5: PV ROOF MONITORS WTH ACTVE HEAT RECOVERY

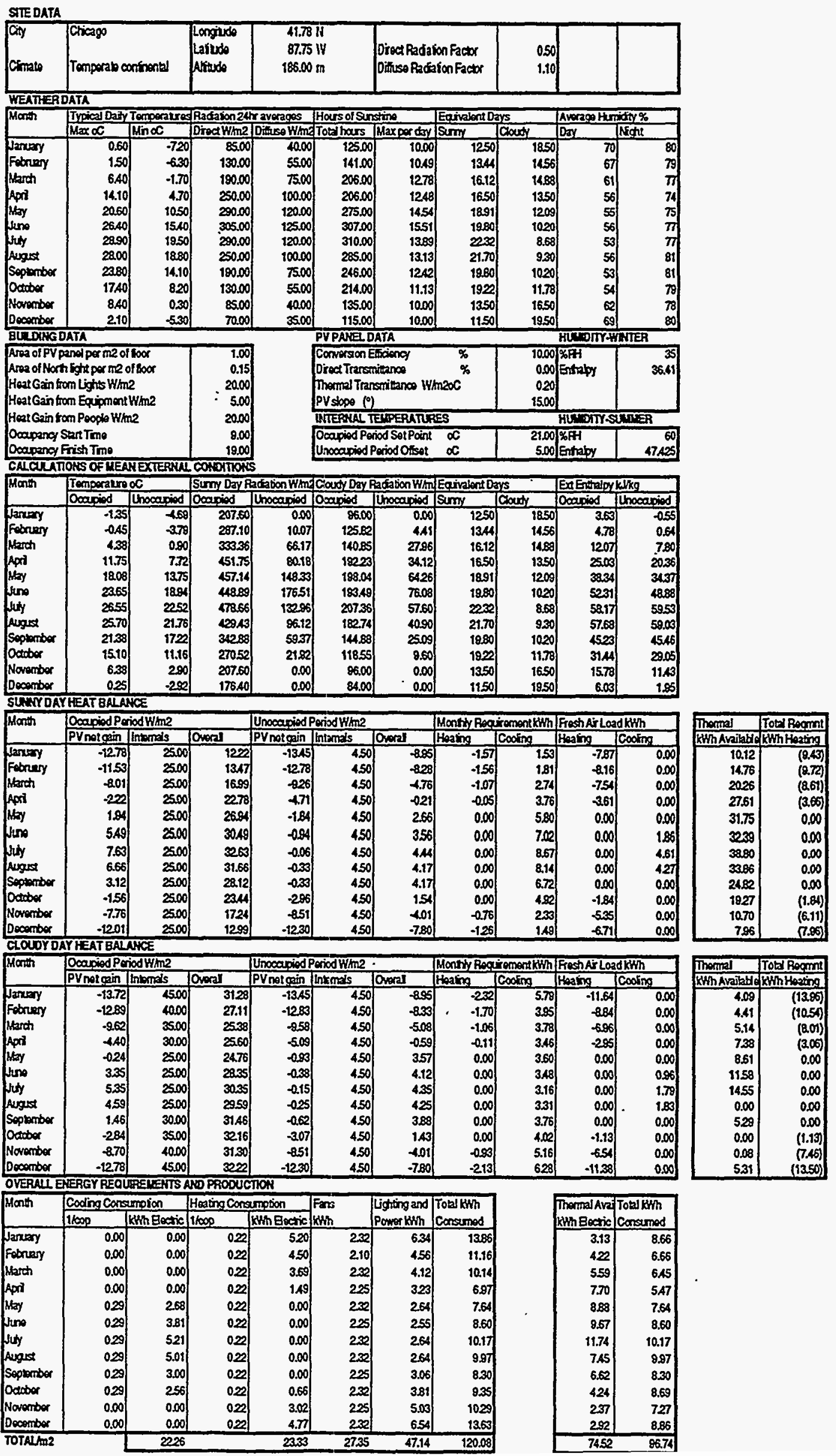


OPTION 5: PV ROOF MONITORS WITH ACTIVE HEAT RECOVERY

\begin{tabular}{|c|c|c|c|c|c|c|c|c|c|c|c|c|}
\hline \multirow{2}{*}{$\begin{array}{l}\text { Sileodia } \\
\text { Cay } \\
\text { chate }\end{array}$} & \multicolumn{2}{|l|}{ Cromali } & $\begin{array}{l}\text { Longlude } \\
\text { Laitude } \\
\text { Alrude }\end{array}$ & $\begin{array}{r}39.15 \\
8452 \\
23200\end{array}$ & & $\begin{array}{l}\text { Drect Radato } \\
\text { Dimuso Pladar }\end{array}$ & $\begin{array}{l}\text { fon Factor } \\
\text { afion Factr }\end{array}$ & $\begin{array}{l}0.50 \\
1.10\end{array}$ & & & & \\
\hline & & & & & & & & & & & & \\
\hline$\sqrt{\text { Mansth }}$ & Iypical Dazy & Tomperatres & Padaion 24 & travergos & Hours of Sins & sine & Equivedent Da & ays & Averega $\mathrm{HW}$ & miofor & & \\
\hline & Max & Mnoc & Drect Hhon 2 & Dansso W/m & Totd hars & Maxperday & simy & Cooudy & Day & I Nodt & & \\
\hline $\begin{array}{l}\text { Januery } \\
\text { Foonery }\end{array}$ & $\begin{array}{r}620 \\
630\end{array}$ & $\begin{array}{r}-330 \\
-290\end{array}$ & $\begin{array}{r}8500 \\
93000\end{array}$ & $\begin{array}{l}40.00 \\
5500\end{array}$ & $\begin{array}{l}108.00 \\
12900\end{array}$ & $\begin{array}{l}10.00 \\
1024\end{array}$ & \begin{tabular}{|l|}
10.85 \\
1260
\end{tabular} & 2020 & tos & \begin{tabular}{|l|l|}
8 & 83 \\
&
\end{tabular} & & \\
\hline March & 11.10 & 0.70 & 190.00 & 7500 & 173.00 & 11.16 & 1550 & 1550 & 57 & 78 & & \\
\hline appit & 1800 & 6.60 & 250.00 & 100.00 & 20100 & 11.96 & 1680 & 1320 & 53 & 76 & & \\
\hline May & 2380 & 11.90 & 200000 & 120.00 & 241.00 & 1274 & 1891 & 1200 & 53 & 78 & & \\
\hline Jino & 2800 & 1720 & 30500 & 125.00 & 305.00 & 14.73 & 20.70 & 930 & 5 & 80 & & \\
\hline Juty & 3080 & 19.10 & 200.00 & 120.00 & 322,00 & $14 / 3$ & 2232 & 8.88 & 52 & 82 & & \\
\hline Nugust & 3020 & 1830 & 25000 & 100.00 & 291.00 & 1341 & 21.70 & 930 & 52 & 86 & & \\
\hline Sopunber & 2600 & 1420 & 190,00 & 75.00 & 25400 & 1215 & 2040 & 9.50 & 50 & 86 & & \\
\hline Dotobor & 2050 & 820 & 130000 & 5500 & 213.00 & 1126 & 1891 & 1209 & ต & 85] & & \\
\hline Norember & 1180 & 220 & 85000 & 40.00 & 14500 & 10.51 & $13 B 0$ & 1620 & 60 & 81 & & \\
\hline Decormber & 5.90 & -230 & 70.00 & 3500 & 126.00 & 10.16 & 1240 & 18.60 & 68 & 82 & & \\
\hline BUTAIHG & DATA & & & & PYPAELLD & & & & HULOTKस & WhIIER & & \\
\hline $\begin{array}{l}\text { Asea of PVP } \\
\text { Area of Nor } \\
\text { Heat Gaint } \\
\text { Heal Gaint }\end{array}$ & 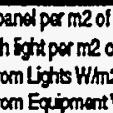 & $\begin{array}{l}\text { Ifoor } \\
\text { of foor } \\
2 \\
\text { Whing }\end{array}$ & \begin{tabular}{r|}
1.00 \\
0.15 \\
2000 \\
5.00
\end{tabular} & & $\begin{array}{l}\text { Conversion } \\
\text { Drect Transm } \\
\text { Thermal Tran } \\
\text { PV stope }\end{array}$ & $\begin{array}{l}\text { Exciancy } \\
\text { nitancos } \\
\text { ssmitanco Wh }\end{array}$ & $\begin{array}{c}\% \\
\% \\
\% 30\end{array}$ & $\begin{array}{r}10.00 \\
0.00 \\
0.20 \\
15.00\end{array}$ & $\begin{array}{l}\text { Fif } \\
\text { Entraby }\end{array}$ & $\begin{array}{r}35 \\
36.41 \\
\end{array}$ & & \\
\hline Hotcaing & on Peopio H/h & & 20.00 & & DNERPUL T & EDPERATLRE & & & HUTOTIS & $\frac{1}{\text { SरTIEA }}$ & & \\
\hline $\begin{array}{l}\text { boupancy } \\
\text { Oocapancy }\end{array}$ & $\begin{array}{l}\text { SutTme } \\
\text { Frish Tme }\end{array}$ & & $\begin{array}{r}9.00 \\
18.00\end{array}$ & & $\begin{array}{l}\text { Ocapied Po } \\
\text { UnocapiodP }\end{array}$ & $\begin{array}{l}\text { nod Sot Point } \\
\text { Period Onsot }\end{array}$ & $\begin{array}{l}\infty \\
\infty\end{array}$ & $\begin{array}{r}21.00 \\
5.00\end{array}$ & FFH & $\begin{array}{r}60 \\
47 A 25\end{array}$ & & \\
\hline CNCOUT & DOHS OF ME & HEXTERAUL & Comotions & & & & & & & & & \\
\hline Herch & Temocrature & & Sum Day $P_{2}$ & $20500 n \mathrm{~W} / \mathrm{m}^{2}$ & Choudy Dar $\mathrm{P}$ & action WhI & Eaivelone & & Extentralpy & $\mid k\}_{k g}$ & & \\
\hline & Oosuied & Throsapiod & Doapiod & Thocalpiod & Docupied & Uhocapiod & Sum $y$ & Couchy & Docaciod & Uhocaroind & & \\
\hline Jaxuery & 383 & -025 & 20760 & 0.00 & 5 $\quad 66.00$ & 0.00 & 10.80 & 2020 & 11.96 & 5. & & \\
\hline Fobruery & 1.00 & 0.06 & 29420 & 5.00 & 123.83 & 2.19 & 1260 & 15.40 & 11.79 & 6.77 & & \\
\hline March & 850 & 4.04 & 381.68 & 31.66 & 16127 & 1338 & 1550 & 1550 & 1891 & 1360 & & \\
\hline Apri & 15.15 & 1026 & $471+10$ & 65.14 & 200.60 & 2314 & 1680 & 1320 & 3124 & 2631 & & \\
\hline Mey & 20.83 & 15.73 & 521.63 & 10226 & 225.98 & 4330 & 1891 & 1200 & 4300 & 4035 & & \\
\hline Juno & 2500 & 20.23 & 47237 & 159.74 & 203.61 & 685 & 20.70 & 930 & 57.64 & 56001 & & \\
\hline Juty & 2788 & 2285 & 46082] & 145.70 & 199.63 & 63.12 & 2232 & 8.68 & 5104 & 0303 & & \\
\hline Augass & 2723 & 2213 & 42058 & 1024 & 17897 & 4359 & 21.70 & 8.30 & 5930 & 2055 & & \\
\hline Sopunbor & 23.65 & 1825 & 36.14 & 5990 & 1457 & 2531 & 20.40 & 9.60 & 4924 & 5029 & & \\
\hline October & 1743 & 1215 & 26740 & 24.14 & 117.19 & 10.58 & 1891 & 1209 & 3290 & 3294 & & \\
\hline Noventber & $9 \neq 0$ & 529 & 19758 & 7.16 & 81.37 & 331 & 1380 & 1620 & 2121 & 1641 & & \\
\hline Decomber & 385 & 0.34 & 173.60 & 200 & 8267 & 0.05 & 1240 & 18.60 & 1200 & 727 & & \\
\hline SLFYOR & YHEATBALA & & & & & & & & & & & \\
\hline Montry & oospiod $\mathrm{Per}$ & nod W/m2 & & ThocapiodP & PeriodW/m2 & & Monthy Regi: & Frementkth II & Frosidirlos & adkth & Thermal & Toter foom \\
\hline & PVnetgin & Intme's & Toweril & PVnotgein & Tinemels & |axed & Heasing & $\operatorname{cosing}$ & Hoaling & TCoofing & kthavalibs & B \\
\hline$\sqrt{\operatorname{lanu} u r y}$ & $-9,42$ & 2500 & 1558 & -1056 & 54 & -506 & -0.29 & 1.68 & 5.07 & 0.00 & $\frac{8.52}{8.20}$ & | \\
\hline Fobruery & -858 & 25.00 & $16 \times 2$ & -1032 & 450 & -582 & -1.03 & 207 & 595 & 0.00 & 14.04 & $(6.8)$ \\
\hline March & +28 & 2500 & 20.08 & -7.51 & 450 & 201 & -0.65 & 3.11 & 521 & 0.00 & 208 & (5.85) \\
\hline Appi & 0.16 & 2500 & $25: 16$ & 3.17 & 450 & 133 & 0.00 & 454 & -1.67 & 0.00 & 29.19 & (1.67) \\
\hline May & 427 & 2500 & 2827 & -0.84 & 450) & 356 & 0.00 & 6.89 & 0.00 & 0.00 & 36.09 & 0.00 \\
\hline Juno & 7.15 & 25.00 & 3215 & -0.87 & 450 & 3.63 & 0.00 & 7.71 & 0.00 & 406 & 3554 & 0.00 \\
\hline Juty & 834 & 25.00 & 33.34 & -0.82 & 4.50 & 3.68 & 0.00 & 859] & 0.00 & 5.83 & 3728 & 0.00 \\
\hline Augess & 758 & 2500 & 3258 & -0.57 & 450 & 3.93 & 0.00 & 826 & 0.00 & 4.96 & 3206 & 0.00 \\
\hline Seplamber & 1.50 & 2500 & 2960 & -0.63 & 450 & 387 & 0.00 & 7.14 & 0.00 & $0.7 \pi$ & 2541 & 0.00 \\
\hline Octuber & -2.08 & 2500 & 24.92 & -230 & 450 & 220 & 0.00 & 530 & 0.00 & 0.00 & 18.65 & 0.00 \\
\hline Noventioer & -5.88 & 2500 & 19.12 & -690 & 450 & -240 & -0.46 & 264 & -103 & 0.00 & 1033 & $(4.49)$ \\
\hline Docombor & -9.69 & 2500 & 1531 & -10.16 & 450 & -5.66 & -0.98 & 190 & -581 & 0.00 & 8.35 & (6.79) \\
\hline CLOLOYO & AY HEATBAL & AHCE & & & & & & & & & & \\
\hline Month & Oogpiod Por & rodw/m2 & & UncocapledP & PeriodW/m2 & & Monthy Regr & Temantkph & Fresthar Lo & & Thermal & Tow Rocm \\
\hline & PV not gain & Insmeds & Ouperal & PVnotgain & Intemals & |Oweral & Hoation & Cocing & Heasing & TCoofing & KWhAvitat & (kWh Heating \\
\hline panuery & -10.36 & 45.00 & 34.64 & -10.56 & 450 & -6.06 & \begin{tabular}{|c|}
-4.77 \\
\end{tabular} & 7.00 & -9.48 & 0.00 & 3.63 & 3. (1120) \\
\hline Fobnery & .9.97| & 40.00 & 32003 & .10 .34 & 450 & -584 & -126 & 4.60 & .728 & 0.00 & 4.43 & (854) \\
\hline Wearch & $-6 . \pi$ & 35.00 & 2320 & -7.66 & 450 & -316 & -0.68 & 438 & 521 & 0.00 & 5.75 & (5.80) \\
\hline Apri & .212 & 30.00 & 2788 & 349 & 450 & 101 & 0.00 & 387 & $-1,31$ & 0.00 & 7.17 & (1.31) \\
\hline May & 1.78 & 2500 & 2678 & $-1 / 13$ & 450 & 3.07 & 0.00 & 3.76 & 0.00 & 0.00 & 10.00 & 0.00 \\
\hline Jino & 4.90 & 2500 & 29.90 & -0.12 & 450 & 438 & 0.00 & 335 & 0.00 & 182 & 1189 & 0.00 \\
\hline Juby & 6.15 & 25.00 & 31.15 & 0.11 & 4.50 & 4.53 & 0.00 & 326 & 0.00 & 227 & 1330 & 0.00 \\
\hline Augest & 5.55 & 25.00 & 30.55 & 0.01 & 4.50 & 451] & 0.00 & $3 / 3$ & 0.00 & 2.13 & 0.00 & 0.00 \\
\hline Soplembor & 294 & 30.00 & 3294 & -0.82 & 450 & 358 & 0.00 & 3.64 & 0.00 & 0.33 & 523 & 0.00 \\
\hline oachere & .134 & 3500 & 33.66 & -241 & 150 & 200 & 0.00 & $4 / 2$ & 0.00 & 0.00 & 0.00 & 0.00 \\
\hline Nowersber & $-6 \pi$ & 40.00 & 3323 & -294 & 450 & -244 & -0.55 & 538 & 4.73 & 0.00 & 0.07 & (528) \\
\hline Decomber & $-10,15$ & 45.00 & 3455 & -10.17 & 450 & -5.67 & $-1,18$ & 6,43 & -8.72 & 0.00 & 5.11 & (10.19) \\
\hline OVERQEIE & NERGYREOU & UAREMENTS $\lambda$ & ANOPAODUC & $\mathrm{THON}$ & & & & & & & & \\
\hline Mantr & Coding Const & sempion & Heasing Conse & ampion & JFans & Lighting and 7 & Tobalkth & & & & Thermal Ave & TOM $\mathrm{kWm}$ \\
\hline & 11000 & kWhBectic & 16000 & ktwh Rectic & $x+4 n$ & Power Kth & Consunod & & & & krm Bectic & mod \\
\hline Jaxeary & 0.00 & 0.00 & 022 & 382 & 245 & 6.68 & 1294 & & & & 259 & 9.12 \\
\hline Fobruary & 0.00 & 0.00 & 022 & 3.45 & 221 & 4.69 & 1035 & & & & 4.05 & 6.90 \\
\hline Warch & 0.00 & 0.00 & 022 & 261 & 245 & 4.19 & 925 & & & & 6.12 & 6.63 \\
\hline Aperd & 028 & $2 \wedge 0$ & 022 & 0.56 & 237 & 321 & 8.64 & & & - & 8.00 & 7.88 \\
\hline May & 0.29 & 292 & 022 & 0.00 & 215 & 2.64 & 8.01 & & & & 10.14 & 8.01 \\
\hline Jine & 0.29 & 484 & 0.22 & 0.00 & 237] & 255 & 9.76 & & & & 10.43 & 9.76 \\
\hline Juby & 0.29 & 5.70 & 022 & 0.00 & 245 & 264 & 10.79 & & & & 11.13 & 10.79 \\
\hline August & 029 & 536 & 022 & 0.00 & 245 & 264 & 10.45 & & & & 728 & 10.45 \\
\hline Seplomber & 0.29 & 3.38 & 022 & 0.00 & 237] & 3.03 & 8.78 & 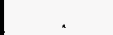 & & & 6.74 & 8.78 \\
\hline oustorer & 029 & 2.78 & 022 & 0.00 & 245 & 384 & 9.07 & & & & 4.10 & 9.07 \\
\hline Nowambor & 0.00 & 0.00 & 022 & 2.17 & 237! & 4.98 & 952 & & & & 220 & 735 \\
\hline Decomber & 0.00 & 0.00 & 022 & 3.78 & 2,s & 6.36 & 1258 & & & & 290 & 8.80 \\
\hline TOTALm2 & & 2739 & & $16, A 9$ & 2883 & 47.42 & 120.14 & & & & 75.96 & 103.65 \\
\hline
\end{tabular}


STEDATA

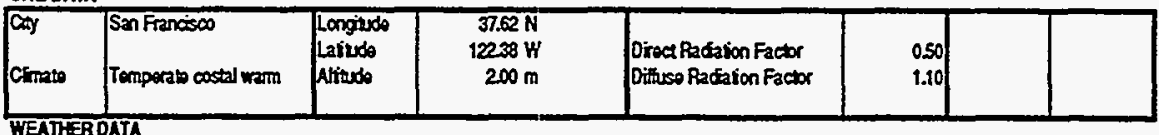

WEATHERDATA

\begin{tabular}{|c|c|c|c|c|c|c|c|c|c|c|}
\hline \multirow[t]{2}{*}{ Mant? } & \multicolumn{2}{|c|}{ Trpical Dain Terpoerares } & \multicolumn{2}{|c|}{ Radaion 24 r avorags } & \multicolumn{2}{|c|}{ Hours of Sunstine } & \multicolumn{2}{|c|}{ Eavialent Days } & \multicolumn{2}{|c|}{ Avereges Hurridy\% } \\
\hline & $\operatorname{Max} \infty$ & $\min \alpha$ & Diect Whin? & 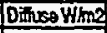 & Tordhars & Maxporday & Surry & Count & Day & Nont \\
\hline Janury & 13.00 & 540 & 8500 & 4000 & 154.00 & 1000 & 1540 & 15.60 & 75 & U \\
\hline abrery & 14.70 & 630 & 130.00 & 55.00 & 191.00 & 1156 & 16.52 & $11 / 48$ & 71 & 8 \\
\hline Warch & 1640 & 720 & 190.00 & 7500 & 262.00 & 1243 & 21.08 & 9.92 & 65 & B \\
\hline Api & 1790 & 8.40 & 250.00 & 100.00 & 297.00 & 1394 & 21.30 & 8.70 & 66 & 8 \\
\hline May & $19 A 0$ & 9.80 & 20000 & 120.00 & 328.00 & 1490 & 22.01 & 8.99 & 66 & 8 \\
\hline hin & 2120 & 1130 & 305.00 & 12500 & 341.00 & 1536 & 2220 & 780 & 65 & 8 \\
\hline haty & 220 & 1190 & 290.00 & 120.00 & 295.00 & 1420 & 20.77 & 1023) & 6 & $\theta$ \\
\hline Augest & 2210 & 1210 & 250.00 & 10000 & 275.00 & 1365 & 20.15 & 10.25 & 69 & 9 \\
\hline Septantox & 2320 & 1220 & 190.00 & 7500 & 275.00 & 1273 & 24.60 & 840 & 66 & 8 \\
\hline Octuber & 21.40 & 1030 & 130.00 & 55.00 & 242.00 & 1131 & 2139 & 2.51 & 65 & 8 \\
\hline Novomber & 1780 & 7.70 & 8500 & 4000 & 198.00 & 10.00 & 19.80 & 1020 & 68 & \\
\hline Decomber & 14.00 & 6.10 & 70.00 & 35,00 & 179.00 & 10.31 & 1736 & 1364 & 74 & \\
\hline \multicolumn{4}{|c|}{ BUDDINGDATA } & & \multicolumn{4}{|c|}{ PVPANEIDATA } & \multicolumn{2}{|c|}{ HLSDTYWLVEA } \\
\hline \multirow{4}{*}{\multicolumn{3}{|c|}{ 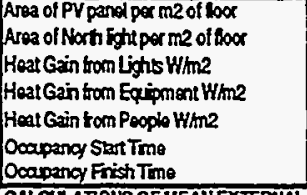 }} & & & \multirow{2}{*}{\multicolumn{3}{|c|}{ 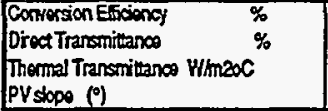 }} & $\begin{array}{c}10.00 \\
0.00\end{array}$ & \multirow{2}{*}{ Entrapy } & \\
\hline & & & $\begin{array}{r}20.00 \\
5.00\end{array}$ & & & & & $\begin{array}{l}0.020 \\
0.00\end{array}$ & & \\
\hline & & & 20.00 & & \multicolumn{4}{|c|}{ INTERNAL TEMPERATWES } & \multicolumn{2}{|c|}{ HURDTTSSTHWER } \\
\hline & & & $\begin{array}{r}9.00 \\
19,00\end{array}$ & & \multicolumn{3}{|c|}{ 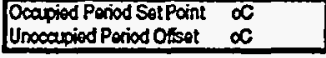 } & $\begin{array}{r}21.00 \\
5,00\end{array}$ & ofit & $\begin{array}{r}6 \\
47.42\end{array}$ \\
\hline \multicolumn{11}{|c|}{ CALCULATOHS OF UEAHEXTERALL COMOITIONS } \\
\hline \multirow[t]{2}{*}{ Manth } & \multicolumn{2}{|c|}{ Temperalro oC } & Sumy Day Rs & action W/m2 & Coudy Day R & gadaionW/ng & Equivent Da: & & Extentralgyk & $\mathrm{k} / \mathrm{kg}$ \\
\hline & Oocaiod & thocapiod & Oosupiod & Unocapiod & Loapiod & Throcapiod & Surny & County & Toosioied & Thocapiod \\
\hline lantary & 11.10 & 7.84 & 207.60 & 0.00 & 8600 & 0.00 & 15.40 & 15.60 & 28001 & 225 \\
\hline abruey & 1250 & 8.00 & 250.51 & 2906 & 114.17 & 1274 & 1652 & 1148 & 30.55 & 25.1 \\
\hline ach & 14.10 & 10.16 & 342.75 & 59.46 & 144.82 & 25.13 & 21.08 & 0.52 & 3271 & 275 \\
\hline pri & 15.53 & $11 / 25$ & 404.48 & 113.24 & 172.12 & 4848 & 2130 & 8.70 & 36.08 & 31.15 \\
\hline tay & 17.00 & 1289 & 446.11 & 15621 & 19326 & 67.67 & 2201 & 899 & 30.5 & $34, B$ \\
\hline Juno & 1873 & 1448] & 453.11 & 17349 & 18531 & 74.78 & 2220 & 780 & 4367 & 39.65 \\
\hline dy & $19 \%$ & 1521 & 488.06 & 140.53 & 2027 & 6028 & 20.77 & 1023 & 47.52 & 426 \\
\hline ugust & 1960 & 1531 & 41326 & 107.57 & 17585 & 4582 & 20.15 & 10.55 & 4755 & 209 \\
\hline opternber & 20.45 & 1574 & 334.60 & 6528 & 14138 & 2758 & 21.60 & 8.40 & 4259 & 43.5 \\
\hline Octaber & 1863 & 12867 & 26623 & $24: 88$ & 116.67 & 10.5 & 2139 & 8.51 & 4312 & 37.7 \\
\hline Noverber & 1528 & 10.95 & 207.60 & 0.00 & 86.00 & 0.00 & 19.80 & 1020 & 36.10 & 22.66 \\
\hline Decontiber & 1203 & 8.64 & 171.08 & 3.60 & 81.47 & 1.81 & 1736 & 13.64 & 29.94 & 245 \\
\hline intring & YFEAT BALA & $\mathrm{MCE}$ & & & & & & & & \\
\hline Uants & Doonoied Per & fodW/nin & & 0 & Whan 2 & & Reger & tkwh & Fresharloa & adkrh \\
\hline & PVnotgin & Thenels & Oweral & PV rat gain & Intrmas & Oxaral & Hoaling & Cooling & Heaing & Cooling \\
\hline Jaxury & -024 & 4500 & 4.76 & -15 & 450 & 287 & 0.00 & 751 & -248 & 0.0 \\
\hline Fobreary & 0.51 & 4500 & 4551 & -1.16 & 450 & 334 & 0.00 & 820 & -1.86 & 0.0 \\
\hline March & 150 & 4500 & 4650 & -0.57 & 450 & 3.83 & 0.00 & 10.53 & -1.50 & 0.06 \\
\hline trii & 230 & $\mathbf{4 5 . 0 0}$ & 4730 & 0.05 & 450 & 455 & 0.00 & $11 / 8$ & -0.14 & $00 x$ \\
\hline yy & 295 & 4500 & 47.95 & 0.36 & 450 & 486 & 0.00 & 1205 & 0.00 & 000 \\
\hline ine & 3.35 & 4500 & 4835 & 0.55 & 450 & 5.15 & 0.00 & 1234 & 0.00 & 0.00 \\
\hline juty & 3.66 & 4500 & 4865 & 0.52 & 450 & $5 . \infty$ & 0.00 & 11.57 & 0.00 & 0.08 \\
\hline Augast & 3.19 & 4500 & 48.19 & 027 & 4,50 & 4.7 & 0.00 & 11.06 & 0.00 & 0.05 \\
\hline Sepromber & 2.70 & 4500 & 47.70 & 0.00 & 450 & 450 & 0.00 & 11.56 & 0.00 & 0.4 \\
\hline patuber & 1.76 & 4500 & 46.76 & -022 & 450 & 428 & 0.00 & 1128 & 0.00 & 0.00 \\
\hline mber & 0.60 & 4500 & 45.60 & -1.01 & 450 & 3.49 & 0.00 & 10000 & -0.12 & 0.00 \\
\hline Decomber & -0.36 & 4500 & 44.64 & $-1 / 4$ & 450 & 306 & 0.00 & $8 / 9$ & -216 & 0.0 \\
\hline
\end{tabular}

CLOOOYOAYPEAT BALAKCE

\begin{tabular}{|c|c|c|c|c|c|c|c|c|c|c|c|}
\hline Manth & Ocapiod Peri & odWhn2 & & UnosomindP & criod Whin & & Month Roqu & irament kth & Frest Ais Load & dkWh & \\
\hline & PVotgin & Intomals & Oworal & PV not cain & Intarals & Ovoral & Heaing & Cooing & Hoging & $\operatorname{cosing}$ & \\
\hline Jaxuay & -1.17 & 4500 & 4383 & -1.53 & 450 & 287 & 0.00 & $7 A 6$ & -252 & & 0.0 \\
\hline$y$ & -0.72 & 4500 & 428 & -120 & 450 & 321 & 0.00 & 5.60 & -120 & & \\
\hline ch & -0.16 & 4500 & 4484 & -0.96 & 450 & 3.54 & 0.00 & 494 & -0.71 & & 0.0 \\
\hline$A x^{2}$ & 0.35 & 45.00 & 4535 & -0.50 & 450 & 4.00 & 0.00 & 4143 & -0.06 & & 0.0 \\
\hline Hay & 0.82 & 4500 & 4582 & -0.05 & 450 & 4.45 & 0.00 & 4.68 & 0.00 & & 0.0 \\
\hline$n$ & 1.19 & 4500 & 46.19 & 0.32 & 450 & 180 & 0.00 & 4.13 & 0.00 & & 0.0 \\
\hline$u\rangle$ & $1 A B$ & 4500 & 46.43 & 0.35 & 450 & 4.85 & 0.00 & 5.4 & 0.00 & & 0.04 \\
\hline August & 120 & 4500 & 4620 & 025 & 450 & 4.75 & 0.00 & 5.73 & 0.00 & & 0.0 \\
\hline Soptriber & 1.08 & 45,00 & 4608 & 0.18 & 450 & 4.68 & 0.00 & 4A2 & 0.00 & & 0.19 \\
\hline October & 0.51 & 45.00 & 45.51 & -0.33 & 450 & 4.17 & 0.00 & 4.93 & 0.00 & & 00 \\
\hline Noventor & -0.34 & 4500 & 4466 & -1.01 & 450 & 3.49 & 0.00 & 5.05 & -0.05 & & 0.0 \\
\hline Docostrber & -1.11 & 45.00 & 4389 & -146 & 450| & 3.04 & 0.00 & 6.57 & -1.69 & & 0.00 \\
\hline
\end{tabular}

OVERAII ENERGY REOUREE RERTS AFO PRODUCTION

\begin{tabular}{|c|c|c|c|c|c|c|c|}
\hline \multirow[t]{2}{*}{ Manth } & \multicolumn{2}{|c|}{ Coding Consumpion } & \multicolumn{2}{|c|}{ Heating Consumpion } & \multirow{2}{*}{$\begin{array}{l}\text { Fans } \\
\text { kHh }\end{array}$} & \multirow{2}{*}{$\begin{array}{l}\text { ligting and } \\
\text { Powor kWh }\end{array}$} & \multirow{2}{*}{$\begin{array}{l}\text { Toul kWh } \\
\text { Consemed }\end{array}$} \\
\hline & 11000 & Khth Bextic & 1 loop & Khin Bectic & & & \\
\hline Jansey & 0.00 & 0.00 & 022 & 1.11 & 3.68 & 8.84 & 13.63 \\
\hline Februay & 0.00 & 0.00 & 022 & 0.70 & 3.33 & 7.98 & 1201 \\
\hline March & 0.00 & 0.00 & 022 & 0.49 & 3.68 & BBA & 13.01 \\
\hline Apri & 0.29 & 453 & 022 & 0.04 & 3.55 & 8.55 & 16.69 \\
\hline May & 0.29 & 4.78 & 022 & 0.00 & 3.68 & 8.84 & 1730 \\
\hline Jung & 029 & 4.70 & 022 & 0.00 & 356 & 8.55 & 16.82 \\
\hline bity & 029 & 489 & 022 & 0.00 & 3.68 & 8.84 & $17 A 1$ \\
\hline August & 0.29 & 4.82 & 0.22 & 0.00 & 3.68 & 8.84 & 17.34 \\
\hline Seprombor & 028 & 4.79 & 022 & 0.00 & 355 & 8.55 & 1600 \\
\hline October & 028 & 4.03 & 022 & 0.00 & 3.68 & 8.84 & 17.15 \\
\hline Noromber & 029 & 4.30 & 022 & 0.04 & 356 & 8.55 & 1645 \\
\hline Decomber & 0.00 & 0.00 & 022 & 0.85 & 3.68 & 8.84 & 1337 \\
\hline TOTALma2 & & 3745 & & 324 & 4336 & 104.03 & 188.08 \\
\hline
\end{tabular}




\section{REF. DESIGN FLAT INSULATED NON-PV ROOF}

STIEDATA

\begin{tabular}{|c|c|c|c|c|c|c|c|c|c|c|}
\hline \multirow{2}{*}{$\begin{array}{l}\text { Cty } \\
\text { CSmlo } \\
\text { WEATHER DA }\end{array}$} & \multicolumn{2}{|c|}{$\begin{array}{l}\text { Now Yark } \\
\text { Tomperab costil ald }\end{array}$} & $\begin{array}{l}\text { Longinds } \\
\text { Lafiuds } \\
\text { Altude }\end{array}$ & $\begin{array}{r}40.70 \\
74.02 \\
3.00\end{array}$ & & $\begin{array}{l}\text { Oirod Rada } \\
\text { Oibuse Pada }\end{array}$ & $\begin{array}{l}\text { ion Factox } \\
\text { aion Factox }\end{array}$ & $\begin{array}{l}0.50 \\
1.10\end{array}$ & & \\
\hline & & & & & & & & & & \\
\hline Month & Typical Daty & Temooralues & Fadaion 24h & Ir averagses & Hours of ST & sting & Egiviont D & gys & Averag Hum & odty \% \\
\hline & $\operatorname{Max} O C$ & Min $\infty$ & Droct W/m? & Dornse What & Totalhars & Waxporday & sumy & Couty & Day & Nigh \\
\hline Januay & 430 & -280 & 85.00 & 40.00 & 15100 & 1000 & 15.10 & 15.00 & 61 & 69 \\
\hline Fabruery & 4.10 & -240 & 130.00 & 55.00 & 16400 & 1028 & 1596 & 1204 & 58 & 67 \\
\hline March & 8.200 & 0.60 & 190.00 & 75.00 & 20900 & 1183 & 17.67 & 1333 & 56 & 67 \\
\hline Apri & 1490 & 6.10 & 250.00 & 100.00 & 21400 & 1209 & 17.70 & 1230 & 55 & 68 \\
\hline $\mathrm{Mry}$ & 20.90 & 11.80 & 290.00 & 120.00 & 250.00 & 13.44 & 18.60 & 1240 & 56 & 69 \\
\hline & 2560 & 1650 & 305.00 & 125.00 & 301.00 & 16.18 & 18.60 & 11.40 & 58 & 72 \\
\hline suly & 2230 & 19.90 & 290.00 & 120.00 & 307,00 & 15.00 & 20.46 & 10.54 & 57 & 74 \\
\hline Augas: & 27A0! & 19.30 & 250.00 & 100.00 & 275.00 & 1386 & 1984 & 11.16 & 60 & 76 \\
\hline Soptomber & 2380 & 1580 & 190.00 & 75.00 & 237.00 & 1254 & 1800 & 11.10 & 60 & 76 \\
\hline actabor & 1840 & 1030 & 130.00 & 5500 & 218.00 & 1134 & 1922 & 11.78 & 59 & 74 \\
\hline Novernber & 11.90 & 440 & 85.00 & 40.00 & 172.00 & 10.06 & 17.10 & 1290 & 60 & 71 \\
\hline December & 5.70 & -120 & 70.00 & 35,00 & 158.00 & 10.00 & 1580 & 1520 & 60 & 67 \\
\hline BUILOINGL & ATA & & & & PVPANEID & ATA & & & HUUDITY'W & NRT \\
\hline $\begin{array}{l}\text { Area of PV } \\
\text { Area of Nor } \\
\text { Heat Gainf } \\
\text { Heat Gainf }\end{array}$ & $\begin{array}{l}\text { and per m2 of } \\
\text { h Light por m2 of } \\
\text { on Lighis W/m2 } \\
\text { om Eqipmant }\end{array}$ & $\begin{array}{l}f \text { foor } \\
\text { of fioor } \\
12 \\
\text { Whin2 }\end{array}$ & $\begin{array}{r}1.00 \\
0.00 \\
20.00 \\
5.00\end{array}$ & & $\begin{array}{l}\text { Conversion E } \\
\text { Direct Transh } \\
\text { Thermal Tran } \\
\text { PY slope (?) } \\
\end{array}$ & $\begin{array}{l}\text { Sticioncy } \\
\text { nitancos } \\
\text { sritancos Wh }\end{array}$ & $\operatorname{mac}^{\%}$ & $\begin{array}{r}10.00 \\
0.00 \\
0.20 \\
0.00 \\
\end{array}$ & FFH & $\begin{array}{r}35 \\
35,41\end{array}$ \\
\hline Heat Gainf & om Pcoplo W/man & & 20.00 & & INTERAUL T & EXPERATUR & & & HLYWDTYSU & UUISER \\
\hline $\begin{array}{l}\text { Oocupancy } \\
\text { Oocapancy }\end{array}$ & $\begin{array}{l}\text { Sturt Tmo } \\
\text { FrishTmo }\end{array}$ & & $\begin{array}{r}9.00 \\
19.00\end{array}$ & & $\begin{array}{l}\text { Ocapiod Po } \\
\text { Unosapiod }\end{array}$ & $\begin{array}{l}\text { rod Sol Poind } \\
\text { Period Otsol }\end{array}$ & $\begin{array}{l}\alpha \\
\infty\end{array}$ & $\begin{array}{r}21.00 \\
5.00\end{array}$ & $\begin{array}{l}\text { \%HH } \\
\text { Entraby }\end{array}$ & $\begin{array}{r}60 \\
47 A 25 \\
\end{array}$ \\
\hline CALCULAT & OAS OFWEA & MEXIERMAL & 2 COADITONA & & & & & & & \\
\hline Monith & Temperature & & Surny Day $R$ & góbon What & Cloudy Day & adaton Win & Equivalont Da & & ExtEntralyle & ellkg \\
\hline & Oocapied & Thocapied & Coapiod & Lhooconiod & Doupiod & thocapiod & Surm & Croudy & Ooapiod II & Uhocapiod \\
\hline Jaxury & 253 & -0.52 & 207.60 & 0.00 & 96.00 & 0.00 & 15.10 & 1590 & 8.95 & 483 \\
\hline Fobreary & 248 & -0.31 & 293.12 & 5.77 & 128.46 & 253 & 15.96 & 1204 & 8.56 & 499 \\
\hline March & 683 & 327 & 360.16 & 47.03 & 152.18 & 1987 & 17.67 & 1333 & 15.67 & 10.86 \\
\hline Apri & 1270 & 8.93 & 466.49 & 69.65 & 188.50 & 28.64 & 17.70 & 1230 & 26.71 & 2180 \\
\hline May & 1863 & 14.78 & 494.61 & 121.56 & 21427 & 5250 & 18.60 & 1240 & 39.98 & 3506 \\
\hline & 23,43 & 19.70 & 430.09 & 189.94 & 18539 & 81.87 & 18.60 & 1140 & 5274 & 49.03 \\
\hline futy & 2620 & 2260 & 443.06 & 15839 & 181.24 & 68.62 & 20.46 & 10.54 & 59.60 & 5833 \\
\hline Augus: & 2538 & 21.90 & 406.20 & 11221 & 173.15 & 47.55 & 19.84 & 11.16 & 59.06 & 57.18 \\
\hline Sepponber & 21.80 & 1837 & 339.72 & 61.63 & 14354 & 26.04 & 1800 & 11.10 & 49.48 & 45.90 \\
\hline Octobser & 1638 & 1290 & 265.55 & 2546 & 116.38 & 11.16 & 1922 & 11.78 & 3584 & 3204 \\
\hline Norember & 10.03 & 681 & 20630 & 0.86 & 0544 & 0.40 & 17.10 & 1290 & 2245 & 18.01 \\
\hline December & 3.98 & 1.02 & 17640 & 0.00 & 84.00 & 0.00 & 15.80 & 1520 & 1126 & 7.09 \\
\hline SLWWYDA & I HEAT BALAH & MCE & & & & & & & & \\
\hline Monith & Oocopiod Peri & rod Whin2 & & UnocouriodP & PeriodWhen & & Month Rog & rementkHh & Fosh Ailload & ddkWh \\
\hline & \begin{tabular}{|l|} 
PVnot qain \\
\end{tabular} & Intenals & Oueral & PVnotgain & Intrinals & Ouard & Hoating & Cooting & Hoaisg lo & Cooling \\
\hline January & -1.95 & $\quad 4500$ & 4305 & 330 & 4.50 & 120 & 0.00 & 6.75 & -7.96 & 0.00 \\
\hline Fobruery & -124 & 4500 & 43.76 & 321 & 4.50 & 129 & 0.00 & 727 & -8.54 & 0.00 \\
\hline March & 0.19 & 4500 & 45.19 & -215 & 450 & 235 & 0.00 & 8.57 & -7.04 & 0.00 \\
\hline Aprit & 226 & 4500 & 4726 & -0.83 & 4.50 & 3.67 & 0.00 & 927 & -330 & 0.00 \\
\hline & 3.68 & 45.00 & 48.68 & -023 & 4.50 & 427 & 0.00 & 10.17 & 0.00 & 0.00 \\
\hline & 4.10 & 45.00 & 49.10 & 0.67 & 450 & 5.17 & 0.00 & 1048 & 0.00 & 1.90 \\
\hline & 4.76 & 4500 & 48.76 & 0.65 & 450 & 5.15 & 0.00 & 11.66 & 0.00 & 4.78 \\
\hline August & 429 & 4500 & 4920 & 0.46 & 450 & 495 & 0.00 & 11.16 & 0.00 & 4.43 \\
\hline Septertiber & 3.01 & 4500 & 48.01 & -0.01 & 4.50 & 449 & 0.00 & 1026 & 0.00 & 0.75 \\
\hline Octubor & 1,31 & 4500 & 4631 & -0.41 & 450 & 4.09 & 0.00 & 10.00 & -0.21 & 0.00 \\
\hline Novertber & -0.46 & 4500 & 4454 & -1.83 & 4.50 & 267 & 0.00 & 826 & 4.58 & 0.00 \\
\hline Decomber & $-1,92$ & 4500 & 4308 & 3.00 & 450 & 1.50 & 0.00 & 7.14 & -7.63 & 0.00 \\
\hline CLOWOY D & TYHEAT BALA & AMCE & & & & & & & & \\
\hline Month & Ocoupiod Peri & riod W/m2 & & Unocapiod P & PeriodW/m2 & & Montity Roge & Sementkwn & FrostAirload & dkWh \\
\hline & PVnotgin & Interpels & Overal & PVnatgain & Intands & Owaral & Hoaing & Cooing & Hoaing & Cooling \\
\hline Januey & -289 & 45.00 & 4211 & 330 & 450 & 120 & 0.00 & 6.96 & -838 & 0.00 \\
\hline Fabruary & -263 & 45.00 & 4237 & 324 & 450 & 126 & 0.00 & 531 & $-6,44$ & 0.00 \\
\hline Marach & -156 & 45.00 & 43.44 & -238 & 450 & 2.12 & 0.00 & 6.19 & -531 & 0.00 \\
\hline Aprit & 0.01 & 45.00 & 45.01 & -1.17 & 450 & 333 & 0.00 & 6.11 & -222 & 0.00 \\
\hline Mxy & 1.32 & 4500 & 4532 & 0.19 & 450 & 4.59 & 0.00 & 6.56 & 0.00 & 0.00 \\
\hline Jino & 2.04 & 45.00 & 47.04 & 0.33 & 450 & 483 & 0.00 & 6.13 & 0.00 & 1.16 \\
\hline Juty & 265 & 45.00 & 47.65 & 0.40 & 450 & 490 & 0.00 & 5.75 & 0.00 & 2.46 \\
\hline August & 233 & 4500 & 4733 & 0.25 & 450 & 4.75 & 0.00 & 6.02 & 0.00 & 249 \\
\hline Soptonber & 1.37 & 45.00 & 4637 & -021 & 450 & 428 & 0.00 & 581 & 0.00 & 0.44 \\
\hline Oatober & 0.05 & 4500 & 45.05 & -0.53 & 450 & 3.97 & 0.00 & 5.96 & -0.13 & 0.00 \\
\hline Noyentor & -139 & 45.00 & 43.61 & -1.83 & 450 & 257 & 0.00 & 6.11 & 3.46 & 0.00 \\
\hline Deconber & -270 & 45.00 & 4230 & 200 & 450 & 1.50 & 0.00 & 6.75 & -734 & 0.00 \\
\hline OVERALE & MERGYREQU & MREMENTS A & ARD PRODUC & TION & & & & & & \\
\hline Month & Coding Const & unoion & Heating Cons & Imotion & Fans & Lighting and & Tob/ Kth & & & \\
\hline & $1 / \operatorname{cop}$ & kWh Bectic & 11000 & KWhEoctic & kish & Poworith & Consumed & & & \\
\hline Jantery & 0.00 & 0.00 & 022 & 3.63 & 3.74 & 8.84 & $162 t$ & & & \\
\hline Fobruery & 0.00 & 0.00 & 022 & 3.33 & 338 & 7988 & 14.60 & & & \\
\hline March & 0.00 & 0.00 & 022 & 2.74 & 3.74 & 8.84 & $15: 32$ & & & \\
\hline Aprit & 0.00 & 0.00 & 022 & 124 & 3.62 & 8.55 & 13.41 & & & \\
\hline $\mathrm{Mry}$ & 029 & 4.78 & 0.22 & 0.00 & 3.74 & 8.84 & 1735 & & & \\
\hline June & 029 & 5.52 & 022 & 0.00 & 3.62 & 855 & 17.79 & & & \\
\hline Juty & 029 & 7.04 & 0.22 & 0.00 & 3.74 & 8.84 & 19.62 & & & \\
\hline August & 029 & 689 & 022 & 0.00 & 3.74 & 8.84 & 19.46 & & & \\
\hline Seprember & 029 & 4.93 & 022 & 0.00 & 3.62 & 8.55 & 17.10 & & & \\
\hline Oateber & 0.29 & 4.56 & 022 & 0.08 & 3.74 & 8.84 & 1721 & & & \\
\hline Noromber & 0.00 & 0.00 & 022 & 1.79 & 3.62 & 855 & 1396 & & & \\
\hline Docomber & 0.00 & 0.00 & 0.22 & 333 & 3.74 & 8.84 & 1590 & & & \\
\hline TOTALm2 & & 3382 & & 16.14 & 44.03 & 104.03 & 198.02 & & & \\
\hline
\end{tabular}




\section{REF. DESIGN FLAT INSULATED NONHPY ROOF}

STEDATA

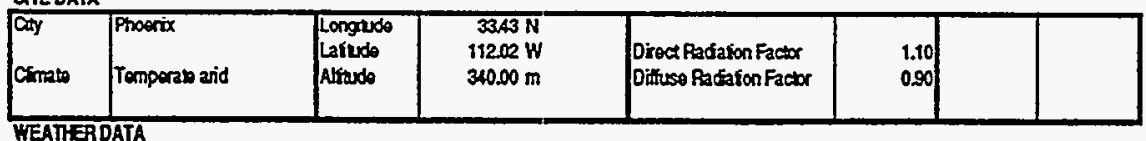

\section{WEATFERDATA}

\begin{tabular}{|c|c|c|c|c|c|c|c|c|c|c|c|}
\hline \multirow[t]{2}{*}{ Nonth } & \multicolumn{2}{|c|}{ Trpical Dati Temporaures } & \multicolumn{2}{|c|}{ Radalion 24tr averagos } & \multicolumn{2}{|c|}{ Hors of Sunstine } & \multicolumn{2}{|c|}{ Equivalent Days } & \multicolumn{3}{|c|}{ Averaco Hunidify\% } \\
\hline & $M a x \propto C$ & $\operatorname{Min} \alpha$ & DiroctWhe & Dinse Hima & Totalhars & Maxporday & sumy & Couty & Day & & Notit \\
\hline andary & 17800 & 1.20 & 105.00 & 2500 & 252.00 & 10.84 & 24.18 & 6.82 & & 44) & $\overline{72}$ \\
\hline$b n$ & 20.10 & 350 & 150.00 & 30.00 & 271.00 & 1241 & 2184 & 6.16 & & 42) & 1 \\
\hline $\operatorname{arch}$ & 2390 & 6.10 & 205.00 & 35.00 & 331.00 & 1256 & 2635 & 4.65 & & 3i) & 60 \\
\hline $\boldsymbol{x}$ & 2880 & 1020 & 260.00 & 45.00 & 360.00 & 1348 & 26.70 & 330 & & 26 & 50 \\
\hline 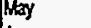 & 3380 & 1390 & 295.00 & 45.00 & 408.00 & 14.00 & 29.14 & 188 & & 19 & 41 \\
\hline n & 38.70 & 1860 & 305.00 & 50.00 & 411.00 & 1457 & 2220 & 180 & & 17) & 37 \\
\hline dy & 4030 & 2390 & 205.00 & 45.00 & 390.00 & 1430 & 2728 & 3.72 & & 29 & 51 \\
\hline gast & 38.70 & 2300 & 260.00 & 45.00 & 379.00 & 1422 & 26.68 & 434 & & 35 & 60 \\
\hline punbor & 36.80 & 1960 & 205.00 & 35.00 & 354.00 & 13.11 & 27,00 & 3.00 & & 29 & 66 \\
\hline xabor & 3040 & 1260 & 150.00 & 30.00 & 323.00 & 11.58 & 2697 & 4.03 & & 30 & $\infty$ \\
\hline lovernber & 2320 & 5.80 & 105.00 & 25.00 & 282.00 & 10.93 & 2580 & 420 & & 33 & 85 \\
\hline Decenter & 1890 & 280 & 90.00 & 25.00 & 260.00 & 10.75 & 24.18 & 6.82 & & 42 & 70 \\
\hline \multicolumn{5}{|c|}{ BULDIHGDATA } & \multicolumn{4}{|c|}{ PVPANEI DATA } & \multicolumn{3}{|c|}{ HULIDTTYWWEER } \\
\hline \multirow{3}{*}{\multicolumn{3}{|c|}{ 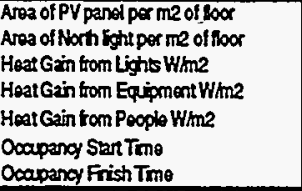 }} & $\begin{array}{r}1.00 \\
0.00 \\
20.00 \\
5.00\end{array}$ & & \multicolumn{3}{|c|}{ 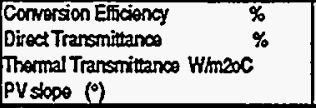 } & $\begin{array}{l}10.00 \\
0.00 \\
0.20 \\
0.00\end{array}$ & \multicolumn{2}{|l|}{$\begin{array}{l}\text { FAt } \\
\text { Enthaby }\end{array}$} & $\begin{array}{r}35 \\
36,41\end{array}$ \\
\hline & & & 20.00 & & \multicolumn{4}{|c|}{ INTERNAL TEMPEAATHRES } & \multicolumn{3}{|c|}{ HUADTTS SUMUEA } \\
\hline & & & $\begin{array}{c}9.00 \\
19.00\end{array}$ & & $\begin{array}{l}\text { Ocaupiad Peri } \\
\text { Unocapiod P }\end{array}$ & $\begin{array}{l}\text { nod Sol Point } \\
\text { Period Olsot }\end{array}$ & $\begin{array}{l}\infty \\
\infty\end{array}$ & $\begin{array}{r}21.00 \\
5.00\end{array}$ & Fot & & $\begin{array}{r}60 \\
47 A 25\end{array}$ \\
\hline
\end{tabular}

CALCULATIOHS OFLEAHEXTERWLL COROTIOHS

\begin{tabular}{|c|c|c|c|c|c|c|c|c|c|c|}
\hline \multirow[t]{2}{*}{ Manth } & \multicolumn{2}{|c|}{ Tomperaere oC } & \multicolumn{2}{|c|}{ Surry Day Radation W/m } & \multicolumn{2}{|c|}{ Cloudy Day Radation W/n } & \multicolumn{2}{|c|}{ EquialontDays } & \multicolumn{2}{|c|}{ IExEntraloy klyg } \\
\hline & Cospiod & Uhocapiod & Ocomind & Unocapiod & Ocoupiod & Uhocaloied & Sumy & Coudy & Dospiod & Uhocanoind \\
\hline January & 1380 & 6.94 & 305.66 & 1824 & 110.75 & 6.61 & 24.18 & 6.82 & 2594 & 18.43 \\
\hline obruay & 16.03 & 9.04 & 371.36 & 6380 & 116.05 & 1896 & 21.84 & 6.16 & 29.56 & 2260 \\
\hline bach & 19,45 & 11.82 & 491.02 & 8984 & 133.74 & 2447 & 2635 & 4.65 & 31.89 & 26.13 \\
\hline pri & 24.15 & 16.18 & 581.17 & 14459 & 160.20 & 3986 & 26.70 & 3.30 & 3782 & 3246 \\
\hline tay & 2883 & 2030 & 625.65 & 178.82 & 15427| & 44,09 & 29.14 & 1.85 & 41.52 & 37.62 \\
\hline ine & 3308 & 2506 & 62658 & 204.73 & 164.67 & 5381 & 2820 & 180 & 47.87 & 45,49 \\
\hline luty & 3620 & 29.17 & 612.75 & 188.04 & 151.09 & 4636 & 2728 & 3.72 & 63.15 & 6382 \\
\hline Lughest & 34.78 & 28.05 & 55121 & 165.99 & 151.94 & 45.76 & 26.66 & 434 & 65.11 & 6653 \\
\hline Sepminber & 3250 & 25.13 & $470 A 4$ & 10454 & 128.14 & 2847 & 27.00 & 3.00 & 5519 & 61.70 \\
\hline Datcotox & 25.95 & 1232 & 384.76 & 5431 & 12024 & 1697 & 2697 & 4003 & 4331 & 40.03 \\
\hline Vorember & 1805 & 11,39 & 303.01 & 20.13 & 109.79 & 729 & 2580 & 420 & 31.61 & 30.54 \\
\hline Decomber & 14.88 & 7.88 & 271.19 & 1458 & 111.60 & 6.00 & 24.18 & 6.82 & 2738 & 2020 \\
\hline
\end{tabular}

SLWY DAYKEAT BALANCE

\begin{tabular}{|c|c|c|c|c|c|c|c|c|c|c|}
\hline Month & Coapiod Pai & iodW/m2 & & Unocospiod P & PeriodW/m2 & & Monthy Rea & Eementkwi I & IFrosh Ai' Load & adkWh \\
\hline & PVnotgin & Intomals & Oueral & PVnatgin & Intomals & Overal & Healing & $\cos \sin x$ & Healing & Cooling \\
\hline Iancry & 1.13 & 4500 & 46.13 & -1.65 & 450 & 284 & 0.00 & 1212 & 486 & 0.00 \\
\hline sbriay & 2.12 & 45,00 & 47.12 & $-A B 6$ & 450 & 3.64 & 0.00 & $11 / 11$ & -208 & 0.00 \\
\hline earch & 381 & 4500 & 4881 & -0.08 & 4.50 & 4,42 & 0.00 & 1419 & -225 & 0.00 \\
\hline xit & 5.51 & 45.00 & 50.51 & 0.25 & 450 & 4.75 & 0.00 & 1526 & 0.00 & 0.00 \\
\hline ay & 682 & 4500 & 5128 & 136 & 4.50 & 586 & 0.00 & 1749 & 0.00 & 0.00 \\
\hline ino & 780 & 45.00 & 5280 & 1.53 & 450 & 6.03 & 0.00 & 1727? & 0.00 & 024 \\
\hline uny & 8.18 & 45.00 & 53.19 & 221 & 450 & 6.71 & 0.00 & 17.07] & 0.00 & 824 \\
\hline Augest & 739 & 45.00 & 5239 & 1.80 & 450 & 6.30 & 0.00 & 1632 & 0.00 & 821 \\
\hline Septatiber & 625 & 4500 & 5125 & 0.70 & 450 & 520 & 0.00 & 1581 & 0.00 & 4.18 \\
\hline Octobs & 422 & 45.00 & 4922 & -0.08 & 4.50 & 4.42 & 0.00 & 14.94 & 0.00 & 0.00 \\
\hline Novamber & 2.12 & 4500 & 47.12 & -0.75 & 450 & 3.75 & 0.00 & 1351 & -238 & 0.00 \\
\hline Decariber & 1.05 & 4500 & 46.05 & -1.48 & 4.50 & 3.02 & 0.00 & 1216 & $-4,19$ & 0.00 \\
\hline
\end{tabular}

CLOLDY DAY HEAT BALAHCE

\begin{tabular}{|c|c|c|c|c|c|c|c|c|c|c|}
\hline \multirow[t]{2}{*}{ Month } & \multicolumn{3}{|c|}{ Oeapiod Period W/m2 } & \multicolumn{3}{|c|}{ Umoocipiod Period W/m2 } & \multicolumn{4}{|c|}{ Monhy PoquromentkWh Frest Ar LoadkWh } \\
\hline & PVnatgain & Inemats & Overal & PVnotgin & Intemals & Ovon: & Heating & $\cos \sin$ & Hoaing & Cooving \\
\hline Januay & -0.51 & 4500 & 44A9 & -1.76 & 450 & 2.74 & 0.00 & 330 & -137 & 0.00 \\
\hline & -0.02 & 4500 & 4498 & -122 & 450 & 328 & 0.00 & 305 & -0.87 & 0.00 \\
\hline march & 0.81 & 4500 & 4581 & -0.63 & 4.50 & 3.87 & 0.00 & 238 & -0.40 & 0.00 \\
\hline Apri & 1.98 & 4500 & 46.98 & -0.13 & 450 & 4.37 & 0.00 & 1.75 & 0.00 & 0.00 \\
\hline Hay & 286 & 4500 & 47.86 & 0223 & 450 & 4.73 & 0.00 & 101 & 0.00 & 0.00 \\
\hline June & 3.92 & 45.00 & 4892 & 0.76 & 450 & 526 & 0.00 & 1.01 & 0.00 & 0.02 \\
\hline Juty & 431 & 45.00 & 49,31 & 1.02 & 4.50 & 5.52 & 0.00 & 2.12) & 0.00 & 1.12 \\
\hline Augh & 4.03 & 45.00 & 49.03 & 0.79 & 450 & 520 & 0.00 & 215 & 0.00 & 150 \\
\hline Soptermber & 338 & 45.00 & 4838 & 0.06 & 450 & 456 & 0.00 & 1.64 & 0.00 & 0.46 \\
\hline Oetobor & 200 & 45.00 & 47.00 & -0.39 & 4.50 & 4.11 & 0.00 & 2.13 & 0.00 & 0.00 \\
\hline November & 0.49 & 45.00 & 4549 & -0.85 & 450 & 3.64 & 0.00 & 2.12 & -0.39 & 0.00 \\
\hline Decentiber & -0.29 & 45.00 & 44.71 & -1.55 & 450 & 295 & 0.00 & 3.33 & $-1,18$ & 0.00 \\
\hline
\end{tabular}

OVERALI EKERGY REQUIREMENTS AYD PRODUCTIOH

\begin{tabular}{|c|c|c|c|c|c|c|c|}
\hline \multirow[t]{2}{*}{ Manth } & \multicolumn{2}{|c|}{ Coding Consermpion } & \multicolumn{2}{|c|}{ Heating Consmotion } & \multirow{2}{*}{$\begin{array}{l}\text { Fans } \\
\text { Kuh }\end{array}$} & \multirow{2}{*}{$\begin{array}{l}\text { Lighting and } \\
\text { Power kWh }\end{array}$} & \multirow{2}{*}{$\begin{array}{l}\text { Total kWh } \\
\text { Consumed }\end{array}$} \\
\hline & 91000 & km Eoctic & $1 / \mathrm{kog}$ & KHm Boctic & & & \\
\hline Jaxuany & 0.00 & 0.00 & 022 & 1.38 & 4.02 & 8.84 & 1424 \\
\hline Fobrary & 029 & 4.13 & 022 & 0.82 & 3.60 & 7.98 & 1656 \\
\hline March & 029 & 4.82 & 022 & 0.60 & 4.02 & 8.84 & 1828 \\
\hline Appril & 029 & 486 & 022 & 0.00 & 3.89 & 8.55 & 1730 \\
\hline $\mathrm{May}$ & 029 & 522 & 022 & 0.00 & 4.02 & 8.84 & 18.14 \\
\hline Jiso & 029 & 530 & 022 & 0.00 & 3.89 & 8.55 & 17774 \\
\hline Juty & 020 & 8.16 & 022 & 0.00 & 4.02 & 8.84 & 21.02 \\
\hline August & 0.20 & 8.42 & 022 & 0.00 & 4.02 & 8.84 & 2128 \\
\hline Soptanber & 029 & 6.31 & 022 & 0.00 & 3.89 & 855 & 18.75 \\
\hline October & 029 & 488 & 0.22 & 0.00 & 4.02 & 8.84 & 17.73 \\
\hline November & 022 & $4 A 7$ & 0.22 & 0.61 & 3.89 & 8.55 & 1752 \\
\hline Decomber & 0.00 & 0.00 & 0.22 & 1.19 & 4.02 & 8.84 & 14.05 \\
\hline TOTAL/m2 & & 56.63 & & 4.61 & 4735 & 104.03 & 212.62 \\
\hline
\end{tabular}


STEDATA

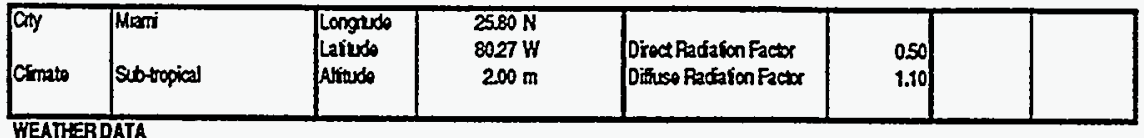

WEATKERDATA

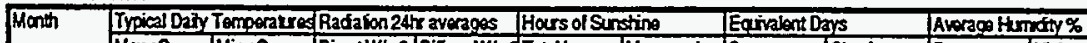

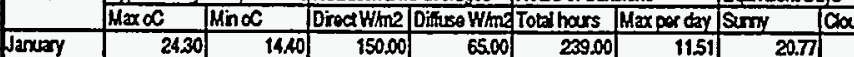

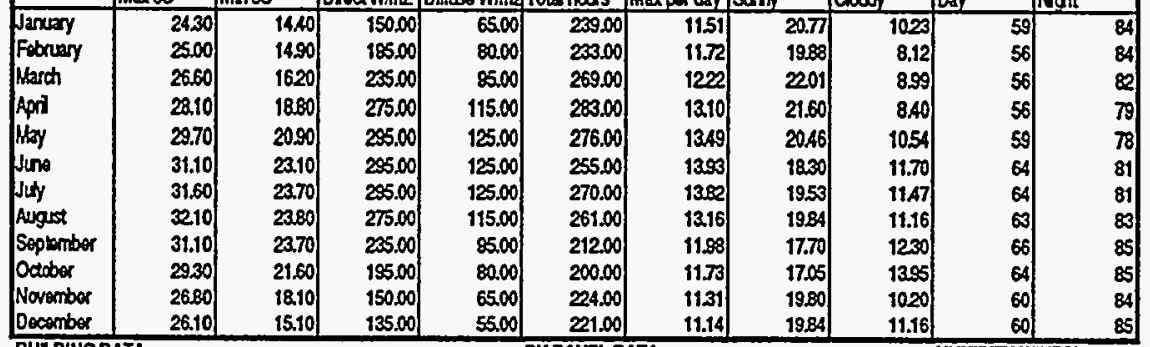
BULDIMGDATA

Aroa of PV panel per me of toor

PYPARELDATA

Area of North ligheper me of toor

Heat Gain forn Lights Whrt

Heal Gain from Equpment Whin

Heat Gain fom Peopio W/m?

Oospancy Sart Tme

1.00
0.00
20.00
5.00
20.00
9.00
19.00

PY PAKEI DATA

CALCLATOHS OF MEANEXTERAL CONDITIONS

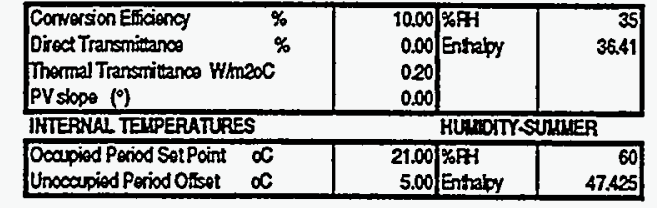

\begin{tabular}{|c|c|c|c|c|c|c|c|c|c|c|}
\hline \multirow[t]{2}{*}{ Manth } & \multicolumn{2}{|c|}{ Temperaturo $\alpha$} & \multicolumn{6}{|c|}{ 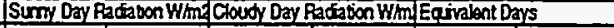 } & \multicolumn{2}{|c|}{ Ext Enthalpy kJ/kg } \\
\hline & Oosioied & Unoocinied & Docupied & Uhrocapiad & osoriod & Uhoocapiad & Simy & Courdy & Ocosiond & Unocapiod \\
\hline Januery & 2183 & 1758 & 32555 & 3089 & 135.57 & 14.59 & 20.77 & 1023 & 49.08 & 4757 \\
\hline Fabrory & 2248 & 18.15 & 37985 & 46.68 & 16382 & 20.13 & 19.88 & 8.12 & 49.32 & 4923 \\
\hline Narch & 24.00 & 19.54 & 435.95 & 69.18 & 18655 & 29.60 & 2201 & 8.99 & 5321 & 5264 \\
\hline Apri & 25.78 & 21.79 & 483.60 & 107.15 & 210.66 & 4657 & 21.60 & 8.40 & 57.88 & 5821 \\
\hline Why & 2750 & 23.73 & 507.05 & 12639 & 22239 & $\mathbf{5 5 . 4 3}$ & 20.46 & 10.54 & 6443 & 6382 \\
\hline Juno & 29.10 & 25.57 & 49087 & 137.35 & 21529 & 60.50 & 1830 & 11.70 & 7243 & 71.86 \\
\hline Juty & 29.63 & 2624 & 454.76 & 135.17 & 217.00 & 5929 & 1953 & $11 A 7$ & 7406 & 7380 \\
\hline Aughast & 30.03 & $26 A 7$ & 481.63 & 108.55 & 209.80 & 4728 & 19.84 & 11.16 & 7460 & 75.78 \\
\hline Septaniber & 2925 & 26.00 & 14ABA & 6283 & 19036 & 2689 & 17.70 & 1230 & 7426 & 7558 \\
\hline Petsoer & 2739 & 24.08 & 379.53 & 45.91 & 163.68 & 2023 & 17.05 & 1395 & 67.18 & 6860 \\
\hline Nowernber & 24.63 & 2050 & 310.79 & 20.15 & 137.89 & 1293 & 19.80 & 1020 & $\mathbf{5 7 . 0 0}$ & 57.67 \\
\hline Decortibor & 2335 & 18.64 & 275.78 & 224 & 118.50 & 8.64 & 19.84 & 11.16 & 5356 & 51.08 \\
\hline
\end{tabular}

SUWWYDAYFEAT BALAHCE

\begin{tabular}{|c|c|c|c|c|c|c|c|c|c|c|}
\hline \multirow[t]{2}{*}{ Manth } & \multicolumn{3}{|c|}{ Deconod Period Whin2 } & \multicolumn{3}{|c|}{ Unocapiod PerrodW/m2 } & \multicolumn{2}{|c|}{ Monfty FogiremontkWh } & \multicolumn{2}{|c|}{ FeshAir Load kWh } \\
\hline & PVnotgain & Inmanals & Overal & PV notgain & Internas & Overal & Hoaing & Cooling & Hoaing & Cooing \\
\hline Januay & 273 & 45.00 & 47.73 & -021 & 450 & 429 & 0.00 & 11.16 & 0.00 & 0.66 \\
\hline Fobray & 3.49 & 4500 & 4249 & 0.02 & 4.50 & 452 & 0.00 & 10.90 & 0.00 & 0.72 \\
\hline March & 428 & 45.00 & 4926 & 0.19 & 4.50 & 4.69 & 0.00 & 1229 & 0,00 & 245 \\
\hline Apri & 5.02 & 4500 & 50.02 & 0.56 & 4.50 & 5.06 & 0.00 & 1233 & 0.00 & 434 \\
\hline Way & 5.56 & 4500 & 5056 & 0.94 & 450 & $5 \mathrm{AM}$ & 0.00 & 11.90 & 0.00 & 6.68 \\
\hline Jine & 5.74 & 4500 & 50.74 & 1.09 & 450 & 559 & 0.00 & 10.72 & 0.00 & 8.78 \\
\hline Juty & 588 & 45.00 & 5088 & 1.18 & 4.50 & 5.68 & 0.00 & 1149 & 0.00 & 8.99 \\
\hline Augest & 5.85 & 4500 & 50.85 & 1.01 & 450 & 551 & 0.00 & 11.02 & 0.00 & 10.35 \\
\hline Septenber & 5.39 & 45.00 & 5030 & 0.54 & 4.50 & 5.04 & 0.00 & 10.17 & 0.00 & 9.12 \\
\hline Octaber & 446 & 45.00 & 49.46 & 0.34 & 450 & 484 & 0.00 & 9.59 & 0.00 & 6.47 \\
\hline Novernber & 334 & 4500 & 4834 & 0.02 & 450 & 452 & 0.00 & 10.82 & 0.00 & 3.64 \\
\hline Decomber & 279 & 45.00 & 47.79 & -0.08 & 450 & $4 A 2$ & 0.00 & 10.71 & 0.00 & 234 \\
\hline
\end{tabular}

CLOUDY DAY HEAT BALANCE

\begin{tabular}{|c|c|c|c|c|c|c|c|c|c|c|}
\hline \multirow[t]{2}{*}{ Marth } & \multicolumn{3}{|c|}{ Ooapiod Penod Whan } & \multicolumn{3}{|c|}{ Unocapied Period W/m2 } & \multicolumn{4}{|c|}{ Monthy RogiremenikWh Fresh Ar LoadkHh } \\
\hline & PVnot gain & Inbmals & loveral & PV netgain & Intonds & Loveral & Hoaing & Cooing & Hoafing & Cooing \\
\hline January & 1,30 & 4500 & 46.30 & -0.06 & 450 & $4 A 4$ & 0.00 & 5.37 & 0.00 & 0.33 \\
\hline Fobruary & 1.67 & 45.00 & 46.67 & -0.07 & 4.50 & 443 & 0.00 & 429 & 0.00 & 0.30 \\
\hline tarch & 2.17 & 4500 & 47.17 & 0.06 & 450 & 456 & 0.00 & 4.81 & 0.00 & 1.00 \\
\hline prî & 272 & 45.00 & 47.72 & 022 & 450 & 4.72 & 0.00 & 4.56 & 0.00 & 1.69 \\
\hline yy & 3.17 & 45.00 & 48.17 & 0.34 & 4.50 & 4.84 & 0.00 & 5.79 & 0.00 & 3.44 \\
\hline ino & 343 & 4500 & 4843 & 0.44 & 450 & 494 & 0.00 & $6 A B$ & 0.00 & 5.62 \\
\hline Juty & 355 & 4500 & 4855 & 0.55 & 450 & 5.05 & 0.00 & 6.38 & 0.00 & 5.86 \\
\hline Augst & 3.57 & 4500 & 4857 & $0.4 \theta$ & 4.50 & 499 & 0.00 & 620 & 0.00 & 5.82 \\
\hline Soplember & 325 & 45.00 & 4825 & 0.24 & 450 & 4.74 & 0.00 & 6.75 & 0.00 & 6.34 \\
\hline Oatober & 265 & 45.00 & 47.65 & 0.12 & 4.50 & 4.62 & 0.00 & 755 & 0.00 & 529 \\
\hline Novernber & 188 & 45.00 & 4688 & -0.01 & 4.50 & 4.49 & 0.00 & 5.42 & 0.00 & 187 \\
\hline December & 1,47 & 45.00 & 46A7 & -0.05 & 4.50 & 4A4 & 0.00 & 5.88 & 0.00 & 1.31 \\
\hline
\end{tabular}

OVERALL ENERGY PEQUREELENTS AKD PRODUCTION

\begin{tabular}{|c|c|c|c|c|c|c|c|}
\hline \multirow[t]{2}{*}{ Month } & \multicolumn{2}{|c|}{ Cooding Consempion } & \multicolumn{2}{|c|}{ Heating Consmemobion } & \multirow{2}{*}{$\begin{array}{l}\text { Fans } \\
\text { kim }\end{array}$} & \multirow{2}{*}{$\begin{array}{l}\text { Highing and } \\
\text { power kth }\end{array}$} & \multirow{2}{*}{$\begin{array}{l}\text { Towlikwh } \\
\text { Consumed }\end{array}$} \\
\hline & $1 / \cos p$ & KWh Boctic & 11000 & kHh $\mathrm{Eectic}$ & & & \\
\hline January & 029 & 5.01 & 022 & 0.00 & 386 & 884 & 17.71 \\
\hline Fabruary & 029 & 4.63 & 022 & 0.00 & 3.49 & 7.98 & 16.10 \\
\hline March & 029 & 5.87 & 022 & 0.00 & 386 & 8.84 & 18.57 \\
\hline Aprit & 029 & 6.55 & 022 & 0.00 & 3.74 & 8.55 & 18.84 \\
\hline$M z^{\prime}$ & 022 & 7.95 & 022 & 0.00 & 3.86 & 8.84 & 20.65 \\
\hline Juno & 029 & 9.03 & 022 & 0.00 & 3.74] & 8.55 & 2132 \\
\hline Juty & 0.29 & 9.63 & 022 & 0.00 & 3.86 & 8.84 & 2233 \\
\hline August & 022 & 9.71 & 0.22 & 0.00 & 3.86 & 8.84 & 2241 \\
\hline Steprmber & 029 & 9.25 & 0.22 & 0.00 & 3.74 & 8.55 & 2154 \\
\hline loctober & 0.29 & 826 & 022 & 0.00 & 3.86 & 884 & 20.95 \\
\hline November & 0.29 & 622 & 022 & 0.00 & 3.74 & 8.55 & 18.51 \\
\hline Docomber & 029 & 5.78 & 0.22 & 0.00 & 3.86 & 8.84 & 18.48 \\
\hline TOTAL $\operatorname{me} 2$ & & 87.88 & & 0.00 & 45.51 & 104.03 & 237.41 \\
\hline
\end{tabular}




\begin{tabular}{|c|c|c|c|c|c|c|c|c|c|c|}
\hline \multirow{2}{*}{$\begin{array}{l}\text { CAy } \\
\text { Crimato } \\
\text { HERTLERDI }\end{array}$} & \multicolumn{2}{|c|}{$\begin{array}{l}\text { Chicago } \\
\text { Tempara coninental }\end{array}$} & \multirow[t]{2}{*}{$\begin{array}{l}\text { Longitudo } \\
\text { Laitude } \\
\text { Alude }\end{array}$} & $\begin{array}{r}41.78 \\
87.75 \\
186.00\end{array}$ & & $\begin{array}{l}\text { Direct Radar } \\
\text { Difuso Rada }\end{array}$ & $\begin{array}{l}\text { ion Factor } \\
\text { ision Factor }\end{array}$ & $\begin{array}{l}0.50 \\
1.10\end{array}$ & & \\
\hline & & & & & & & & & & \\
\hline Manth & Typical Da] I & Terrooritres & Radaion 24h & $x$ arroges & Hars of Sunst & $\sin \theta$ & Eariatentor & ys & Avereg Him: & idity $\%$ \\
\hline & $\operatorname{Max} \infty$ & Minoc & Droct Wing & Diurso W/ma & Total hass & Maxporday & sumy & Cavody & Day & Nist \\
\hline January & 0.60 & -720 & 8500 & 4000 & 125.00 & 1000 & 1250 & 1850 & 70 & 80 \\
\hline Fabreary & 1.50 & -830 & 130.00 & 5500 & 141.00 & 10.48 & 1344 & 14.56 & 67 & 79 \\
\hline March & 610 & -1.70 & 190.00 & 7500 & 206.00 & 1278 & 16.12 & 1488 & 6i) & $\pi$ \\
\hline Apri & 14.10 & 4.70 & 250.00 & 100.00 & 206.00 & 1248 & 1650 & 1350 & 56) & 74 \\
\hline May & 20.60 & 10.50 & 200.00 & 120.00 & 275.00 & 1454 & 1891 & 1209 & 55 & 75 \\
\hline Jino & 2640 & 1540 & 305.00 & 12500 & 307.00 & 15.51 & 19.80 & 1020 & 56 & $\pi$ \\
\hline bety & 2090 & 19.50 & 290.00 & 120.00 & 310.00 & 1389 & 2232 & 8.68 & 53 & $\pi$ \\
\hline August & 2800 & 1880 & 250.00 & 100.00 & 285.00 & 13.13 & 21.70 & 930 & 56 & 81 \\
\hline Septumber & 2380 & 14.10 & 190.00 & 75.00 & 246.00 & 1242 & 19.80 & 1020 & 53 & 81 \\
\hline Datober & 1740 & 8.20 & 130.00 & 55,00 & 214.00 & 11.93 & 1922. & 11.78 & 54 & 79 \\
\hline Novernber & 8.40 & 0.30 & 85.00 & 40.00 & 135.00 & 10.00 & 1350 & 1650 & 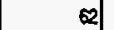 & 78 \\
\hline Docember & 2.10 & -5.30 & 7000 & 35.00 & 115.00 & 10.00 & 11.50 & 19.50 & 69 & 80 \\
\hline BURDINGD & ATA & & & & PYPNEIDA & ATA & & & HUMoITYW. & NTER \\
\hline $\begin{array}{l}\text { Area of PVP } \\
\text { Area ofNort } \\
\text { Heat Gain th } \\
\text { Heat Gain th }\end{array}$ & $\begin{array}{l}\text { and per m2 of } \\
\text { inght per me of } \\
\text { orn Lights W/m2 } \\
\text { om Equipment }\end{array}$ & $\begin{array}{l}\text { lloor } \\
\text { of foor } \\
2 \\
\text { Whan }\end{array}$ & $\begin{array}{r}1.00 \\
0.00 \\
20.00 \\
5.00\end{array}$ & & $\begin{array}{l}\text { Convorsion Efl } \\
\text { Direct Transmi } \\
\text { Thermal Trans: } \\
\text { PVosope (o) }\end{array}$ & $\begin{array}{l}\text { Strioncy } \\
\text { xitanco } \\
\text { smitanco Wh }\end{array}$ & $\begin{array}{c}\% \\
\% \\
m\end{array}$ & $\begin{array}{r}10.00 \\
0.00 \\
0.20 \\
0.00 \\
\end{array}$ & $\begin{array}{l}\text { \%FH } \\
\text { Entrapy }\end{array}$ & $\begin{array}{r}35 \\
3641\end{array}$ \\
\hline Heat Gain f & on Peoplo W/ins & & 2000 & & INTERNAL IE & EIPESATURI & & & HULDTITSUI & MLER \\
\hline $\begin{array}{l}\text { Oocapancy } \\
\text { Docapancy }\end{array}$ & $\begin{array}{l}\text { Sant Tme } \\
\text { Frish Tme }\end{array}$ & & $\begin{array}{r}8.00 \\
19.00\end{array}$ & & $\begin{array}{l}\text { Doapiod Pofio } \\
\text { Unoosipiod Pe }\end{array}$ & $\begin{array}{l}\text { iod Sot Point } \\
\text { oriod Otsol }\end{array}$ & $\begin{array}{l}\infty \\
\infty\end{array}$ & $\begin{array}{r}21.00 \\
5.00\end{array}$ & $\begin{array}{l}\text { FमH } \\
\text { Entraby }\end{array}$ & $\begin{array}{r}60 \\
47,125 \\
\end{array}$ \\
\hline CALCULAT & TOHSOFMEAM & MEXTERMAL & conditnons & & & & & & & \\
\hline Manth & Temporatroo & $\alpha$ & Sumy Day R & GeabionW/int & Coudy Day Ra & 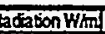 & Eqrivalont Da & & Ext Entraloy $k$ & k $1 \mathrm{~kg}_{\mathrm{kg}}$ \\
\hline & Ocopiod It & Unocousiod & Ocaniod & Uhoosuriod & Oapoind IL & Urrocapiod & Surny & Cloody & Oocaried L & Urocapiod \\
\hline Janutay & -1.35 & 469 & 207.50 & 0.00 & 8600 & 0.00 & 1250 & 1850 & 3.63 & -0.55 \\
\hline Fobreary & -0.45 & -379 & 287.10 & 10.07 & 12582 & 441 & 13.4 & 1456 & 4.78 & 0.64 \\
\hline March & 438 & 0.90 & 333.36 & 66.17 & 140.85 & 2796 & 16.12 & 1488 & 1207 & 780 \\
\hline Apri & 11.75 & 7.72 & 451.75 & 80.18 & 19223 & 34.12 & 16.50 & 1350 & 2503 & 2036 \\
\hline Nay & 18.08 & 13.75 & 457.14 & 148.23 & 198.04 & 6426 & 1899 & 1200 & 3834 & 3437 \\
\hline & 23.65 & 1894 & 448.69 & 176.51 & $193 \mathrm{AO}$ & 76.08 & 19.80 & 1020 & 5231 | & 4888 \\
\hline futy & 26.55 & 2252 & 478.66 & 13206 & 20736 & 57.60 & 2230 & 8.68 & 58.17 & 59.53 \\
\hline August & 25.70 & 21.76 & 40913 & 86.12 & 182.74 & 4080 & 21.70 & 8,30 & 57.68 & 59.08 \\
\hline Septartber & 2138 & 1722 & 34288 & 5937 & 144.68 & 25.00 & 19.80 & 1020 & 4523 & 45.46 \\
\hline Octobor & 1510 & 11.16 & 270.52 & 21.32 & 11855 & 9.60 & 1922 & 11.78 & 31,4 & 2005 \\
\hline Narenber & 638 & 2.90 & 207.60 & 0.00 & 9600 & 000 & 1350 & 1650 & 1578 & 1143 \\
\hline Decernber & 025 & -298 & 176.40 & 0.00 & 84.00 & 0.00 & 1150 & 19.50 & 6.00 & 1.85 \\
\hline SUTHY DA & T HEAT BALAK & KCE & & & & & & & & \\
\hline Menth & 000000 Perio & $\operatorname{cod} \mathrm{W} / \mathrm{m} 2$ & & Unocodiodp & griod W/m2 & & Montry Regu & Tementk & Fresth Á Load & Idwh \\
\hline & PV nos gain & Intonals & Oucral & PVnot gain & Intrna's & Orall & Haging & $\operatorname{cosin}$ & Hoaing & cooing \\
\hline Janury & -273 & 4500 & 4227 & 4.14 & 450 & 035 & 0.00 & 5.35 & -787 & 0.00 \\
\hline Fobruary & -1.88 & 4500 & 43.12 & 387 & 450 & 0.03 & 0.00 & 591) & -8.9 & 0.00 \\
\hline March & -0.52 & 4500 & 448 & -246 & 450 & 204 & 0.00 & 7.58 & -754 & 0.00 \\
\hline Aprit & 1.94 & 45.00 & 4694 & 208 & 450 & 352 & 0.00 & 8.56 & 3.61 & 0.00 \\
\hline Nay & 325 & 4500 & 4825 & 0.30 & 450 & 4.80 & 0.00 & 1039 & 0.00 & 0.00 \\
\hline hos & 430 & 4500 & 4030 & 0.74 & 450 & 524 & 0.00 & 1121 & 0.00 & 1.86 \\
\hline Juty & 5.13 & 4500 & 50.13 & 0.75 & 450 & 525 & 0.00 & 1280 & 0.00 & 4.61 \\
\hline August & 455 & 4500 & 4955 & 0.46 & 450 & 496 & 0.00 & 1226 & 0.00 & $42 \pi$ \\
\hline Soplember & 2.96 & 4500 & 4796 & 024 & 450 & 4.74 & 0.00 & 1081 & 0.00 & 0.00 \\
\hline Octcber & 1.09 & 4500 & 4609 & -0.78 & 450 & 3.72 & 0.00 & 8.86) & -1.84 & 0.00 \\
\hline Noramber & -1.18 & 45.00 & 4382 & -262 & 450 & 188 & 0.00 & 627 & $-5 x$ & 0.00 \\
\hline Decomber & -267 & 45.00 & 4233 & -3.78 & 4.50 & 0.72 & 0.00 & 498 & 6.71 & 0.00 \\
\hline CLOUDYOA & YYEAT BALA & AHCE & & & & & & & & \\
\hline Manth & Ocapied Perio & od Hime & & Unocapiodp & eciodW/h2 & & Monthy Rogi & TementkWh & Frosh Árload & dkwh \\
\hline & PVnetgin 1 & Intmals & Oroal & PVnet gin & Internals & Oronal & Hoafre & $\operatorname{cosin}$ & Heaing IC & cosing \\
\hline Janary & 3.66 & 45.00 & 4134 & 4.14 & 450 & 035 & 0.00 & 7.74 & -11.64 & 0.00 \\
\hline Fabray & 323 & 4500 & 41.7 & -392 & 450 & 0.58 & 0.00 & 620 & 884 & 0.00 \\
\hline March & -214 & 45.00 & 4286 & -278 & 4.50 & 1.72 & 0.00 & 6.73 & 696 & 0.00 \\
\hline Apti & -024 & 45.00 & 4.76 & $-1,37$ & 450 & 3.13 & 0.00 & 6.63 & .295 & 0.00 \\
\hline itay & 1.08 & 4500 & 4508 & 0.00 & 4.50 & 459 & 0.00 & 635 & 0.00 & 0.00 \\
\hline Jino & 2.16 & 4500 & 47.16 & 0.33 & 450 & 483 & 0.00 & 5.50 & 0.00 & 096 \\
\hline Juty & 285 & 45.00 & 47.ES & 0.20 & 450 & 4.79 & 0.00 & 4.74 & 0.00 & 1.78 \\
\hline August & 247 & 4500 & $47 A 7$ & 0.96 & 4.50 & 4.66 & 0.00 & 5.2 & 0.00 & 1.83 \\
\hline Soptomber & 129 & 45.00 & 4629 & -0.05 & 450 & 4.45 & 0.00 & 536 & 0.00 & 0.00 \\
\hline October & -0.18 & 4500 & 488 & -0.89 & 4.50 & 361 & 0.00 & 588 & -1.13 & 0.00 \\
\hline Norember & -212 & 4500 & 4288 & .262 & 4.50 & 1.88 & 0.00 & 751 & -6.54 & 0.00 \\
\hline Decormbor & -344 & 1500 & 41.56 & 3.78 & 450 & 0.72 & 0.00 & 830 & -1138 & 0.00 \\
\hline OVERAL E & HRGYREQU & REIEITS A & RODUCI & 4 & & & & & & \\
\hline Menth & Coding Consur & impion & Hogting Conse & unobion & Fans & Lighing and & Topalkm & & & \\
\hline & 1000 & Khth Eectic & $1 / 600$ & knth Boctic & kth & Powrer kith & Consermed & & & \\
\hline Jamery & 0.00 & 0.00 & 022 & 434 & 3.76 & 884 & 1699 & & & \\
\hline Fobruxy & 0.00 & 0.00 & 022 & 378 & 3.39 & 7.98 & 15.15 & & & \\
\hline March & 0.00 & 0.00 & 022 & 322 & 3.76 & 884 & 1581 & & & \\
\hline Apri & 0.00 & 0.00 & 0.22 & $1 A 6$ & 3.63 & 8.55 & 13.64 & & & \\
\hline Nay & 029 & 4.78 & 022 & 0.00 & 3.76 & 8.84 & 1737 & & & \\
\hline $\operatorname{Jin} \theta$ & 0.29 & 558 & 022 & 0.00 & 3.63 & 8.55 & 17.76 & & & \\
\hline Juty & 029 & 6.85 & 022 & 0.00 & 3.76 & 8.84 & 19.44 & & & \\
\hline August & 0.29 & 6.68 & 022 & 0.00 & 3.76 & 8.84 & 1927 & & & \\
\hline Soptember & 0.29 & 4.62 & 022 & 0.00 & 3.63 & 8.55 & 1680 & & & \\
\hline October & 029 & 450 & 022 & 0.66 & 3.76 & 8.84 & 17.74 & & & \\
\hline Novembor & 0.00 & 0.00 & 022 & 264 & 3.63 & 8.55 & 14.82 & & - & \\
\hline Decombor & 0.00 & 0.00 & 022 & $4 . \infty$ & 3.76 & 8.84 & 16.61 & & & \\
\hline TOTAL/m2 & & 33.00 & & 20.11 & 4421 & 104.03 & 20135 & & & \\
\hline
\end{tabular}


STIEDATA

\begin{tabular}{|c|c|c|c|c|c|c|}
\hline Cory & $\begin{array}{l}\text { Cincrmal } \\
\text { Temperab coninental }\end{array}$ & $\begin{array}{l}\text { Longado } \\
\text { Larido } \\
\text { Atitude }\end{array}$ & $\begin{array}{l}39.15 \mathrm{~N} \\
8152 \mathrm{~W} \\
232.00 \mathrm{~m}\end{array}$ & 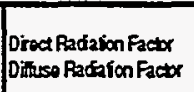 & $\begin{array}{l}0.50 \\
1.10\end{array}$ & \\
\hline
\end{tabular}

\section{WEATHERDATA}

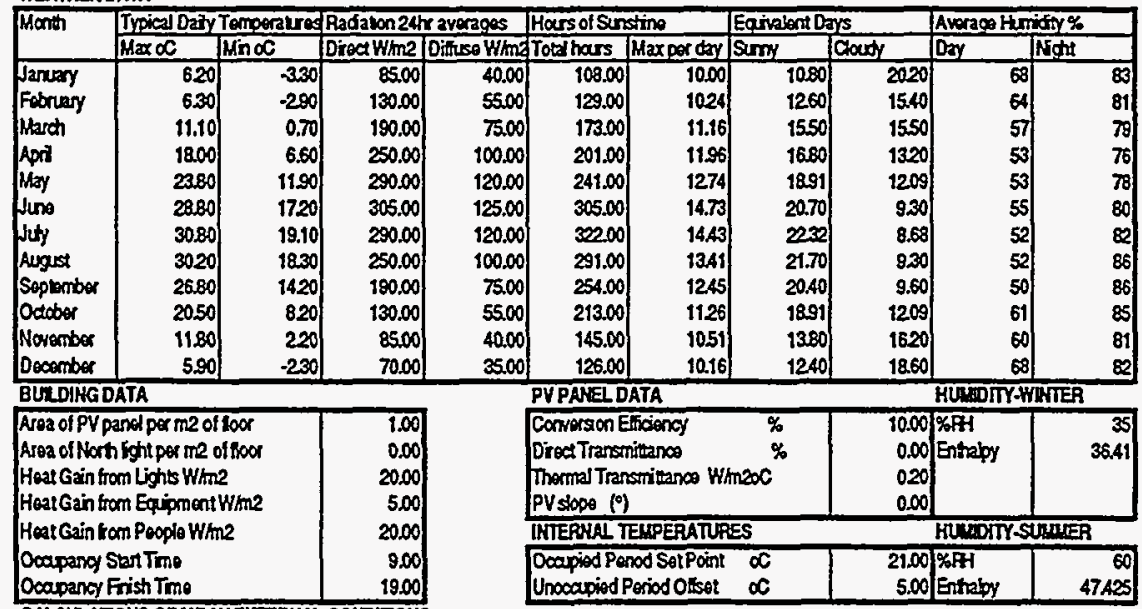

CALCUAATONS OF WEAN EXTERNAL COHDITONS

\begin{tabular}{|c|c|c|c|c|c|c|c|c|c|c|}
\hline \multirow[t]{2}{*}{ Month } & \multicolumn{2}{|c|}{ Temporatrooc } & \multirow{2}{*}{\multicolumn{2}{|c|}{ 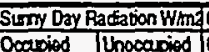 }} & \multicolumn{2}{|c|}{ 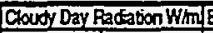 } & \multicolumn{2}{|c|}{ auvalont Dars } & \multicolumn{2}{|c|}{ Exerestragykdikg } \\
\hline & Doaviod & Unocosiod & & & Dosoind & Uhocaniod & Sumy & Couty & Doanied & throcaniod \\
\hline tary & 3.83 & .025 & 207.60 & 0.00 & 96,00 & 0.00 & 1080 & 2020 & 11.96 & 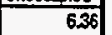 \\
\hline wery & 0 & 0.06 & 29420 & 5.00 & 128.93 & 2.19 & 1260 & 15.40 & 11.79 & \\
\hline ach & 50 & 4.04 & 381.58 & 31.66 & 16127 & 1338 & 1550 & 15,50 & 1891 & \\
\hline & 15.15 & 1026 & 47140 & 66.14 & 200.60 & 28.14 & 1680 & 1320 & 3124 & \\
\hline Mz & 20.83 & 15.73 & 521.60 & 10226 & 22588 & 4430 & 1891 & 1209 & 43.98 & \\
\hline N & 25.90 & 20.93 & 47237 & 159.74 & 203.61 & 6865 & 20.70 & 930 & 57.64 & \\
\hline 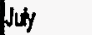 & 27.88 & 2286 & 46082 & 145.70 & 189.60 & 02.12 & 2232 & 8.68 & 61.04 & \\
\hline st & 2723 & 2213 & 4 & 10244 & 17897 & 4359 & 21.70 & 930 & 5938 & \\
\hline entor & 23.65 & 1825 & & $50 \mathrm{ml}$ & 14457] & 2531 & 2040 & 9.50 & & \\
\hline & 17 A3 & 1215 & & 14] & & 058 & 1891 & 1209 & 3898 & \\
\hline & & 529 & 19? & 7.16 & 9137 & 331 & 1380 & 1620 & 2121 & \\
\hline & 3.85 & & 173.60 & & & & 1240] & 1860 & 1200 & \\
\hline
\end{tabular}

SURHY DAY HEAT BALAHCE

\begin{tabular}{|c|c|c|c|c|c|c|c|c|c|c|}
\hline \multirow[t]{2}{*}{ Month } & \multicolumn{2}{|c|}{ Ocapied Period W/m2 } & \multirow[b]{2}{*}{ Oreared } & \multicolumn{2}{|c|}{ Unocapiod Poriod W/m2 } & \multirow[b]{2}{*}{ Overal } & \multicolumn{4}{|c|}{ 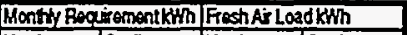 } \\
\hline & PV nol gain I & & & PVotgain & Intmals & & Heaing & Cooing & Heating & Cooling \\
\hline Jaxtary & -1.69 & 45.00 & 4331 & .325 & 450 & $\sqrt{125}$ & 0.00 & 487 & 5.07 & \\
\hline Fabreary & -0.93 & 45,00 & 4.07] & -3.15 & 450 & 1.35 & 0.00 & 5.79 & -5.96 & \\
\hline March & 0.74 & 45.00 & 457.71 & -213 & 450 & 237 & 0.00 & 7.60 & -521 & 0.0 \\
\hline & 2.79 & 45.00 & 47.79 & -0.59 & 450 & 3.91 & 0.00 & 8.95 & -1.67 & \\
\hline Hay & 4.35 & 45.00 & 4935 & 0.30 & 450 & 480 & 0.00 & 10.50) & 0.00 & a \\
\hline Jine & 4.55 & 45.00 & 4995 & 0.56 & 450 & 5.16 & 0.00 & 1183 & 0.00 & 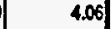 \\
\hline 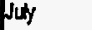 & 525] & 45.00 & 5025 & 0.50 & 450 & 5.10 & 0.00 & 1281 & 0.00 & | \\
\hline gest & 4.78 & 45.00 & 49.78 & 0.42 & 450 & $4: 2$ & 0.00 & 1230 & 0.00 & \\
\hline Sopterbor & $3 A 0$ & 45. & 40) & 0.15 & 450 & 4.65 & 0.00 & 1120 & 0.00 & ) \\
\hline bor & 153 & 4500 & 46.53 & -0.57 & 450 & 393 & 0.00 & 8B4 & 0.00 & 0. \\
\hline mber & -0.66 & 45.00 & 4434 & -208 & 450 & 242 & 0.00 & 6.59 & -4.03 & 0. \\
\hline anbor & -1.97 & 45.00 & 43.03 & -3.12 & 450 & 138 & 0.00 & 5.58 & -581 & \\
\hline
\end{tabular}

CLOCOY DAY HEAT BALANCE

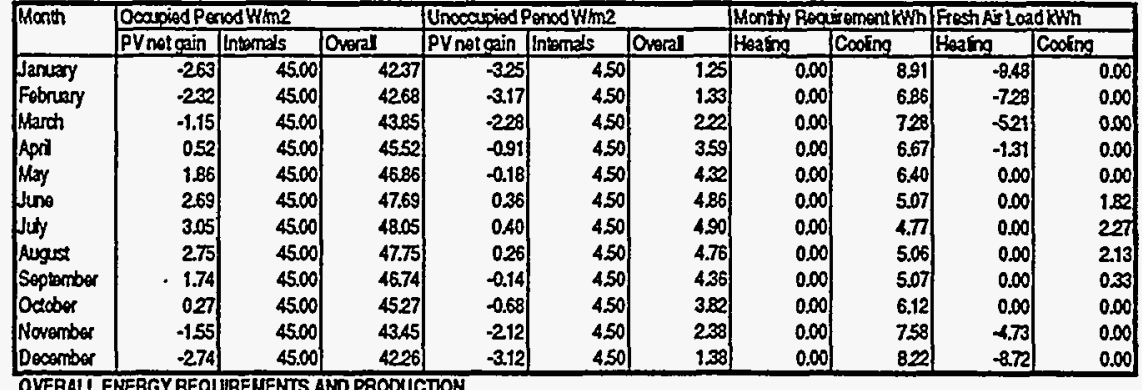

\begin{tabular}{|c|c|c|c|c|c|c|c|}
\hline \multirow[t]{2}{*}{ Menth } & \multicolumn{2}{|c|}{ Coding Consempron } & \multicolumn{2}{|c|}{ Hibaing Consempsion } & \multirow{2}{*}{$\begin{array}{l}\text { Fans } \\
\text { kth }\end{array}$} & \multirow{2}{*}{$\begin{array}{l}\text { Lighing and } \\
\text { Poworkthrn }\end{array}$} & \multirow{2}{*}{$\begin{array}{l}\text { To:al kHWh } \\
\text { Corsumed }\end{array}$} \\
\hline & 11000 & When Boctic & 11000 & kWh Boctic & & & \\
\hline jaxwary & 0.00 & 0.00 & 022 & 325 & 3.80 & 8.24 & 15.87 \\
\hline Fobruary & 0.00 & 0.00 & 022 & 254 & 3.4 & 798 & 1436 \\
\hline अrch & 0.00 & 0.00 & 022 & 232 & 380 & 8.84 & 1495 \\
\hline pil & 029 & $4 A 6$ & 022 & 0.66 & 3.68 & 855 & 1736 \\
\hline by & 029 & 486 & 022 & 0.00 & 380 & 8.84 & 1750 \\
\hline Jine & 029 & 651 & 0.22 & 0.00 & 3.68 & 855 & 18.74 \\
\hline Pury & 029 & 7.34 & 022 & 0.00 & 380 & 884 & 1998 \\
\hline & 029 & 6.98 & 022 & 0.00 & 380 & 8.84 & 19.62 \\
\hline mbor & 029 & 195 & 022 & 0.00 & 3.68 & 8.55 & 17.18 \\
\hline & 029 & 456 & 022 & 0.00 & 380 & 8.84 & 1720 \\
\hline & 0.00 & 0.00 & 022 & 1.95 & 3.68 & 855 & 14.18 \\
\hline sconber & 0.00 & 0.00 & 022 & 323 & 3.80 & 8.84 & 15.87 \\
\hline TOTAL/m2 & & 39.66 & & 14,33 & 44.79 & 104.03 & 20281 \\
\hline
\end{tabular}


D PV HOURLY PERFORMANCE \& UTILITY RATE ANALYSIS 


\begin{tabular}{|c|c|c|c|c|c|c|c|c|c|c|c|c|}
\hline UTILITY RATES & OAKLAND & & & & & & & & & & & \\
\hline \multicolumn{13}{|l|}{ Pacific Gas \& Electric } \\
\hline Rate A6 & Jan & Feb & Mar & Apr & May & Jun & Jul & Aug & Sep & Oct & Nov & Dec \\
\hline 56 & 0.0608 & 0.0608 & 0.0608 & 0.0608 & 0.0789 & 0.0789 & 0.0789 & 0.0789 & 0.0789 & 0.0789 & 0.0608 & 0.0608 \\
\hline 6.7 & 0.0608 & 0.0608 & 0.0608 & 0.0608 & 0.0789 & 0.0789 & 0.0789 & 0.0789 & 0.0789 & 0.0789 & 0.0608 & 0.0608 \\
\hline $7-8$ & 0.0608 & 0.0608 & 0.0608 & 0.0608 & 0.0789 & 0.0789 & 0.0789 & 0.0789 & 0.0789 & 0.0789 & 0.0608 & 0.0608 \\
\hline 8.9 & 0.0809 & 0.0809 & 0.0809 & 0.0809 & 0.1516 & 0.1516 & 0.1516 & 0.1516 & 0.1516 & 0.1516 & 0.0809 & 0.0809 \\
\hline $9-10$ & 0.0809 & 0.0809 & 0.0809 & 0.0809 & 0.1516 & 0.1516 & 0.1516 & 0.1516 & 0.1516 & 0.1516 & 0.0809 & 0.0809 \\
\hline $10-11$ & 0.0809 & 0.0809 & 0.0809 & 0.0809 & 0.1516 & 0.1516 & 0.1516 & 0.1516 & 0.1516 & 0.1516 & 0.0809 & 0.0809 \\
\hline $11-12$ & 0.0809 & 0.0809 & 0.0809 & 0.0809 & 0.1516 & 0.1516 & 0.1516 & 0.1516 & 0.1516 & 0.1516 & 0.0809 & 0.0809 \\
\hline $12 \cdot 13$ & 0.0809 & 0.0809 & 0.0809 & 0.0809 & 0.3029 & 0.3029 & 0.3029 & 0.3029 & 0.3029 & 0.3029 & 0.0809 & 0.0809 \\
\hline 13.14 & 0.0809 & 0.0809 & 0.0809 & 0.0809 & 0,3029 & 0.3029 & 0.3029 & 0.3029 & 0.3029 & 0.3029 & 0.0809 & 0.0809 \\
\hline $14-15$ & 0.0809 & 0.0809 & 0.0809 & 0.0809 & 0.3029 & 0.3029 & 0.3029 & 0.3029 & 0.3029 & 0.3029 & 0.0809 & 0.0809 \\
\hline $15-16$ & 0.0809 & 0.0809 & 0.0809 & 0.0809 & 0.3029 & 0.3029 & 0.3029 & 0.3029 & 0.3029 & 0.3029 & 0.0809 & 0.0809 \\
\hline $16-17$ & 0.0809 & 0.0809 & 0.0809 & 0.0809 & 0.3029 & 0.3029 & 0.3029 & 0.3029 & 0.3029 & 0.3029 & 0.0809 & 0.0809 \\
\hline$\overline{17.18}$ & 0.0809 & 0.0809 & 0.0809 & 0.0809 & 0.3029 & 0.3029 & 0.3029 & 0.3029 & 0.3029 & 0.3029 & 0.0809 & 0.0809 \\
\hline $18-19$ & 0.0800 & 0.0809 & 0.0809 & 0.0809 & 0.1516 & 0.1516 & 0.1516 & 0.1516 & 0.1516 & 0.1516 & 0.0809 & 0.0809 \\
\hline & & & & & & & & & & & & \\
\hline \multicolumn{13}{|l|}{ PV OUTPUT (Option 1, PV1) } \\
\hline \multicolumn{13}{|l|}{ PER HOUR } \\
\hline & & & & & & & & & & & & \\
\hline Oak/15\%/45 az & $\operatorname{Jan}$ & Feb & Mar & Apr & May & Jun & Jul & Aug & Sep & Oct & Nov & Dec \\
\hline 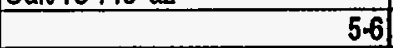 & 0.0000 & 0.0000 & 0.0000 & 0.0000 & 0.1034 & 0.1396 & 0.1233 & 0.0376 & 0.0000 & 0.0000 & 0.0000 & 0.0000 \\
\hline 6.7 & 0.0000 & 0.0000 & 0.0861 & 0.1974 & 0.3254 & 0.3722 & 0.3521 & 0.2442 & 0.1238 & 0.0000 & 0.0000 & 0.0000 \\
\hline $7-8$ & 0.0675 & 0.1616 & 0,3813 & 0.6092 & 0.8528 & 0.9171 & 0.9047 & 0.7096 & 0.4542 & 0.2271 & 0.0899 & 0.0421 \\
\hline 8.9 & 0.3702 & 0.5462 & 0.9620 & 1.2720 & 1.5388 & 1.5679 & 1.5945 & 1.3964 & 1.0706 & 0.7239 & 0.4211 & 0.3208 \\
\hline $9-10$ & 0.8188 & 1.0422 & 1.5841 & 1.9220 & 2.1583 & 2.1555 & 2.2165 & 2.0410 & 1.7135 & 1.3365 & 0.9024 & 0.7493 \\
\hline $10-11$ & 1.2338 & 1.4677 & 2.0936 & 2.4264 & 2.6425 & 2.6110 & 2.7007 & 2.5434 & 2.2307 & 1.8457 & 1.3397 & 1.1541 \\
\hline $11-12$ & 1.5031 & 1.7364 & 2.4118 & 2.7408 & 2.9397 & 2.8890 & 2.9972 & 2.8544 & 2.5517 & $2.16 \pi$ & 1.6248 & 1.4141 \\
\hline $12-13$ & 1.5753 & $1.8+22$ & 2.5007 & 2.8301 & 3.0243 & 2.9698 & 3.0831 & 2.9425 & 2.6418 & 2.2580 & 1.7003 & 1.4898 \\
\hline $13-14$ & 1.4423 & 1.6810 & 2.3510 & 2.6874 & 2.8902 & 2.8455 & 2.9511 & 2.8018 & 2.4955 & 2.1072 & 1.5648 & 1.3577 \\
\hline $14-15$ & 1.1272 & 1.3693 & 1.9871 & 2.3341 & 2.5563 & 2.5342 & 2.6201 & 2.4525 & 2.1341 & 1.7396 & 1.2422 & 1.0477 \\
\hline $15-16$ & 0.6993 & 0.9314 & 1.4654 & 1.8138 & 2.0618 & 2.0707 & 2.1281 & 1.9382 & 1.6095 & 1.2180 & 0.7979 & 0.6289 \\
\hline $16-17$ & 0.2483 & 0.4483 & 0.8618 & 1.1937 & 1.4599 & 1.5049 & 1.5276 & 1.3157 & 0.9962 & 0.6303 & 0.3185 & 0.1960 \\
\hline 17-18 & 0.0000 & 0.0000 & 0.2764 & 0.5629 & 0.8149 & 0.8858 & 0.8765 & 0.6692 & 0.3841 & 0.0000 & 0.0000 & 0.0000 \\
\hline \multirow[t]{2}{*}{$18-19$} & 0.0000 & 0.0000 & 0.0000 & 0.0000 & 0.2416 & 0.3167 & 0.2872 & 0.1234 & 0.0000 & 0.0000 & 0.0000 & 0.0000 \\
\hline & 9.0858 & 11.1963 & 16.9613 & 20.5898 & 23.6099 & 23.7799 & 24.3627 & 22.0699 & 18.4057 & 14.2540 & 10.0016 & 8.4005 \\
\hline Peak kW & 0.0508 & 0.0647 & 0.0807 & 0.0943 & 0.0976 & 0.0990 & 0.0995 & 0.0949 & 0.0881 & 0.0728 & 0.0567 & 0.0481 \\
\hline demand credit @ & $\$ 0.0000$ & $\$ 0.0000$ & $\$ 0.0000$ & $\$ 0.0000$ & $\$ 0.0000$ & $\$ 0.0000$ & $\$ 0.0000$ & $\$ 0.0000$ & $\$ 0.0000$ & $\$ 0.0000$ & $\$ 0.0000$ & $\$ 0.0000$ \\
\hline \multicolumn{13}{|l|}{ Eominam vili } \\
\hline Total energy charges/yr & $\$ 35.3447$ & & & & & & & & & & & \\
\hline Total demand charges/y? & $\$ 0.0000$ & & & & & & & & & & & \\
\hline Total kWh/m2/yr & 202.72 & & & & & & & & & & & \\
\hline Av. SkWh & $\$ 0.1744$ & & & & & & & & & & & \\
\hline & & & & & & & & & & & & \\
\hline
\end{tabular}




\begin{tabular}{|c|c|c|c|c|c|c|c|c|c|c|c|c|}
\hline UTILITY RATES & NEW YORK & & & & & & & & & & & \\
\hline ULCO & & & & & & & & & & & & \\
\hline Rates SC 2-MRP Rate 1 & Jan & Feb & Mar & Apr & May & Jun & Jul & Aug & Sep & Oct & Nov & Dec \\
\hline 策 & 0.1003 & 0.1003 & 0.1003 & 0.1003 & 0.1003 & 0.1038 & 0.1038 & 0.1038 & 0.1038 & 0.1003 & 0.1003 & 0.1003 \\
\hline $6-7$ & 0.1003 & 0.1003 & 0.1003 & 0.1003 & 0.1000 & 0.1038 & 0.1038 & 0.1038 & 0.1038 & 0.1003 & 0.1003 & 0.1003 \\
\hline 78 & 0.1195 & 0.1195 & 0.1195 & 0.1195 & 0.1195 & 0.1230 & 0.1230 & 0.1230 & 0.1230 & 0.1195 & 0.1195 & 0.1195 \\
\hline $8-9$ & 0.1195 & 0.1195 & 0.1195 & 0.1195 & 0.1195 & 0.1230 & 0.1230 & 0.1230 & 0.1230 & 0.1195 & 0.1195 & 0.1195 \\
\hline $9-10$ & 0.1195 & 0.1195 & 0.1195 & 0.1195 & 0.1195 & 0.1230 & 0.1230 & 0.1230 & 0.1230 & 0.1195 & 0.1195 & 0.1195 \\
\hline $10-11$ & 0.1195 & 0.1195 & 0.1195 & 0.1195 & 0.1195 & 0.1375 & 0.1375 & 0.1375 & 0.1375 & 0.1195 & 0.1195 & 0.1195 \\
\hline $11 \cdot 12$ & 0.1195 & 0.1195 & 0.1195 & 0.1195 & 0.1195 & 0.1375 & 0.1375 & 0.1375 & 0.1375 & 0.1195 & 0.1195 & 0.1195 \\
\hline $12-13$ & 0.1195 & 0.1195 & 0.1195 & 0.1195 & 0.1195 & 0.1375 & 0.1375 & 0.1375 & 0.1375 & 0.1195 & 0.1195 & 0.1195 \\
\hline 13.14 & 0.1195 & 0.1195 & 0.1195 & 0.1195 & 0.1195 & 0.1375 & 0.1375 & 0.1375 & 0.1375 & 0.1195 & 0.1195 & 0.1195 \\
\hline $14-15$ & 0.1195 & 0.1195 & 0.1195 & 0.1195 & 0.1195 & 0.1375 & 0.1375 & $0: 1375$ & 0.1375 & 0.1195 & 0.1195 & -0.1195 \\
\hline 15-16 & 0.1195 & 0.1195 & 0.1195 & 0.1195 & 0.1195 & 0.1375 & 0.1375 & 0.1375 & 0.1375 & 0.1195 & 0.1195 & 0.1195 \\
\hline $16-17$ & 0.1195 & 0.1195 & 0.1195 & 0.1195 & 0.1195 & 0.1375 & 0.1375 & 0.1375 & 0.1375 & 0.1195 & 0.1195 & 0.1195 \\
\hline $17-18$ & 0.1195 & 0.1195 & 0.1195 & 0.1195 & 0.1195 & 0.1375 & 0.1375 & 0.1375 & 0.1375 & 0.1195 & 0.1195 & 0.1195 \\
\hline $18-19$ & 0.1195 & 0.1195 & 0.1195 & 0.1195 & 0.1195 & 0.1375 & 0.1375 & 0.1375 & 0.1375 & 0.1195 & 0.1195 & 0.1195 \\
\hline & & & & & & & & & & & & \\
\hline PV OUTPUT (Option 1, PVI) & & & & & & & & & & & & \\
\hline PERHOUR & & & & & & & & & & & & \\
\hline & & & & & & & & & & & & \\
\hline NY/1 $5^{\circ} / 45^{\circ} a z$ & Jan & Feb & Mar & Apr & May & Jun & Jul & Aug & Sep & Oct & Nov & Dec \\
\hline $5 \cdot 6$ & 0.0000 & 0.0000 & 0.0000 & 0.0044 & 0.1157 & 0.1508 & 0.1333 & 0.0475 & 0.0000 & 0.0000 & 0.0000 & 0.0000 \\
\hline 6-7 & 0.0000 & .0 .0000 & 0.0766 & 0.1940 & 0.3220 & 0.3593 & 0.3396 & 0.2361 & 0.1143 & 0.0000 & 0.0000 & 0.0000 \\
\hline $7-8$ & 0.0371 & 0.1304 & 0.3201 & 0.5042 & 0.7075 & 0.7385 & 0.7235 & 0.5718 & 0.3712 & 0.1859 & 0.0582 & 0.0109 \\
\hline 8.9 & 0.2692 & 0.4135 & 0.7349 & 0.9543 & 1.1785 & 1.1662 & 1.1739 & 1.0269 & 0.7913 & 0.5481 & 0.2863 & 0.2071 \\
\hline $9-10$ & 0.6050 & 0.7764 & 1.1775 & 1.3961 & 1.6059 & 1.5543 & 1.5818 & 1.4562 & 1.2283 & 0.9983 & 0.6027 & 0.4854 \\
\hline 10.11 & 0.9200 & 1.0946 & 1.5433 & 1.7426 & 1.9396 & 1.8545 & 1.8983 & 1.7903 & 1.5822 & 1.3826 & 0.8950 & 0.7499 \\
\hline $11-12$ & 1.1238 & 1.2971 & 1.7709 & 1.9551 & 2.1421 & 2.0353 & 2.0896 & 1.9945 & 1.8003 & 1.6246 & 1.0818 & 0.9243 \\
\hline 12-13 & 1.1787 & 1.3479 & 1.8282 & 2.0089 & 2.1938 & 2.0816 & 2.1384 & 2.0462 & 1.8565 & 1.6904 & 1.1295 & 0,9665 \\
\hline 13.14 & 1.0666 & 1.2407 & 1.7088 & 1.8993 & 2.0914 & 1.9897 & 2.0417 & 1.9419 & 1.7455 & 1.5676 & 1.0271 & 0.8669 \\
\hline $14-15$ & 0.8145 & 0.9973 & 1.4336 & 1.6424 & 1.8482 & 1.7717 & 1.8118 & 1.6956 & 1.4835 & 1.2802 & 0.7934 & 0.6491 \\
\hline 15-16 & 0.4767 & 0.6588 & 1.0438 & 1.2712 & 1.4929 & 1.4517 & 1.4749 & 1.3380 & 1.1097 & 0.8780 & 0.4814 & 0.3589 \\
\hline $16-17$ & 0.1333 & 0.2947 & 0.5991 & 0.8350 & 1.0656 & 1.0652 & 1.0688 & 0.9114 & 0.6789 & 0.4340 & 0.1581 & 0.0712 \\
\hline 17.18 & 0.0000 & 0.0000 & 0.1803 & 0.3973 & 0.6125 & 0.6485 & 0.6330 & 0.4733 & 0.2562 & 0.0000 & 0.0000 & 0.0000 \\
\hline 18-19 & 0.0000 & 0.0000 & 0.0000 & 0.0371 & 0.2060 & 0.2618 & 0.2350 & 0.1002 & 0.0000 & 0.0000 & 0.0000 & 0.0000 \\
\hline$\$ 0.1304$ & 6.6249 & 8.2514 & 12.4171 & 14.8419 & 17.5217 & 17.1291 & 17.3436 & 15.6299 & 13.0179 & 10.5897 & 6.5135 & 5.2902 \\
\hline & & & & & & & & & & & & \\
\hline PeakkW & 0.0380 & 0.0481 & 0.0590 & 0.0670 & 0.0708 & 0.0694 & 0.0690 & 0.0660 & 0.0619 & 0.0545 & 0.0377 & 0.0312 \\
\hline demand credit 6 & $\$ 0.0390$ & $\$ 0.0494$ & $\$ 0.0605$ & $\$ 0.0687$ & $\$ 0.0726$ & $\$ 0.0712$ & $\$ 0.0708$ & $\$ 0.0677$ & $\$ 0.0635$ & $\$ 0.0559$ & $\$ 0.0386$ & $\$ 0.0320$ \\
\hline $20 \%$ & & & & & & & & & & & & \\
\hline Total energy charges/yr & $\$ 18.2465$ & & & & & & & & & & & \\
\hline Total demand charges/yr & $\$ 0.6900$ & & & & & & & & & & & \\
\hline Total kWh/m2/yr & 145.17 & & & & & & & & & & & \\
\hline Av. \$NWh & $\$ 0.1304$ & $\cdot$ & & & & & & & & & & \\
\hline & & & & & & & & & & & & \\
\hline
\end{tabular}




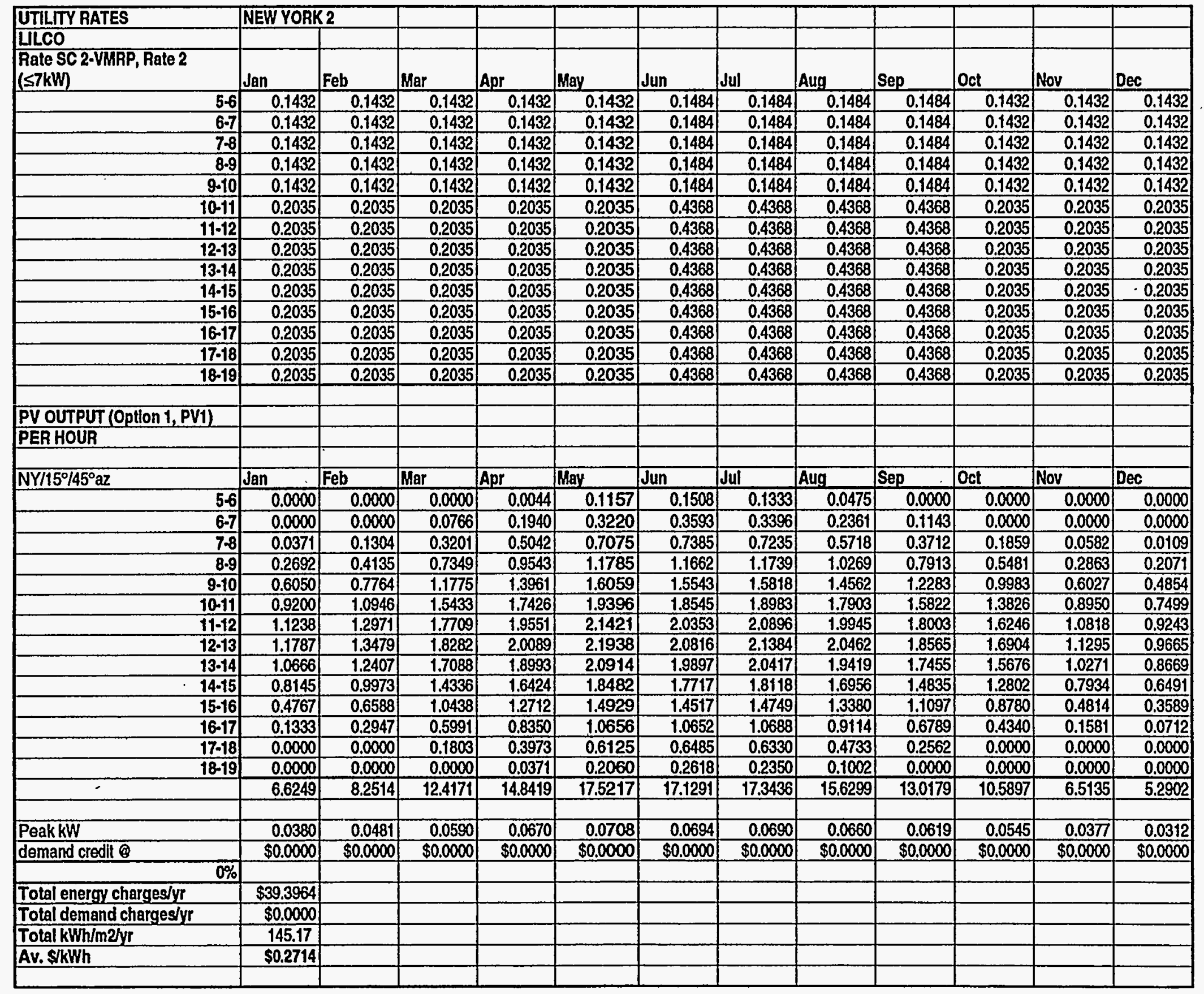




\begin{tabular}{|c|c|c|c|c|c|c|c|c|c|c|c|c|}
\hline UTILTYRATES & PHOENIX & & & & & & & & & & & \\
\hline \multicolumn{13}{|c|}{ Arizona Publlc Service Company } \\
\hline Rate E-32 & Jan & Feb & Mar & Apr & May & Jun & Jul & Aug & Sep & Oet & Nov & Dec \\
\hline 56 & 0.0878 & 0.0878 & 0.0878 & 0.0878 & 0.0878 & 0.0878 & 0.0878 & 0.0878 & 0.0878 & 0.0878 & 0.0878 & 0.0878 \\
\hline 6.7 & 0.0878 & 0.0878 & 0.0878 & 0.0878 & 0.0878 & 0.0878 & 0.0878 & 0.0878 & 0.0878 & 0.0878 & 0.0878 & 0.0878 \\
\hline $7-8$ & 0.0878 & 0.0878 & 0.0878 & 0.0878 & 0.0878 & 0.0878 & 0.0878 & 0.0878 & 0.0878 & 0.0878 & 0.0878 & 0.0878 \\
\hline $8 \cdot 9$ & 0.0878 & 0.0878 & 0.0878 & 0.0878 & 0.0878 & 0.0878 & 0.0878 & 0.0878 & 0.0878 & 0.0878 & 0.0878 & 0.0878 \\
\hline $9-10$ & 0.0878 & 0.0878 & 0.0878 & 0.0878 & 0.0878 & 0.0878 & 0.0878 & 0.0878 & 0.0878 & 0.0878 & 0.0878 & 0.0878 \\
\hline $10-11$ & 0.0878 & 0.0878 & 0.0878 & 0.0878 & 0.0878 & 0.0878 & 0.0878 & 0.0878 & 0.0878 & 0.0878 & 0.0878 & 0.0878 \\
\hline $11-12$ & 0.0878 & 0.0878 & 0.0878 & 0.0878 & 0.0878 & 0.0878 & 0.0878 & 0.0878 & 0.0878 & 0.0878 & 0.0878 & 0.0878 \\
\hline $12-13$ & 0.0878 & 0.0878 & 0.0878 & 0.0878 & 0.0878 & 0.0878 & 0.0878 & 0.0878 & 0.0878 & 0.0878 & 0.0878 & 0.0878 \\
\hline $13 \cdot 14$ & 0.0878 & 0.0878 & 0.0878 & 0.0878 & 0.0878 & 0.0878 & 0.0878 & 0.0878 & 0.0878 & 0.0878 & 0.0878 & 0.0878 \\
\hline $14-15$ & 0.0878 & 0.0878 & 0.0878 & 0.0878 & 0.0878 & 0.0878 & 0.0878 & 0.0878 & 0.0878 & 0.0878 & 0.0878 & 0.0878 \\
\hline $15-16$ & 0.0878 & 0.0878 & 0.0878 & 0.0878 & 0.0878 & 0.0878 & 0.0878 & 0.0878 & 0.0878 & 0.0878 & 0.0878 & 0.0878 \\
\hline $16-17$ & 0.0878 & 0.0878 & 0.0878 & 0.0878 & 0.0878 & 0.0878 & 0.0878 & 0.0878 & 0.0878 & 0.0878 & 0.0878 & 0.0878 \\
\hline $17-18$ & 0.0878 & 0.0878 & 0.0878 & 0.0878 & 0.0878 & 0.0878 & 0.0878 & 0.0878 & 0.0878 & 0.0978 & 0.0878 & 0.0878 \\
\hline 18.19 & 0.0878 & 0.0878 & 0.0878 & 0.0878 & 0.0878 & 0.0878 & 0.0878 & 0.0878 & 0.0878 & 0.0878 & 0.0878 & 0.0878 \\
\hline & & & & & & & & & & & & \\
\hline PV OUTPUT (Option 1, PV1) & & & & & & & $\dot{5}$ & & & & & \\
\hline \multicolumn{13}{|l|}{ PER HOUR } \\
\hline & & & & & & & . & & & & & \\
\hline AZ/15\%/45a & Jan & $\overline{F e b}$ & Mar & Apr & May & Jun & Jul & Aug & $\overline{\operatorname{sep}}$ & Oct & Nov & Dec \\
\hline $5 \cdot 6$ & 0.0000 & 0.0000 & 0.0000 & 0.0000 & 0.0735 & 0.1037 & 0.0898 & 0.0170 & 0.0000 & 0.0000 & 0.0000 & 0.0000 \\
\hline 6.7 & 0.0000 & 0.0000 & 0.0938 & 0.1831 & 0.2793 & 0.3123 & 0.2985 & 0.2176 & 0.1210 & 0.0121 & 0.0000 & 0.0000 \\
\hline $7-8$ & 0.1132 & 0.2102 & 0.4407 & 0.6531 & 0.8758 & 0.9032 & 0.8407 & 0.6950 & 0.4834 & 0.2770 & 0.1342 & 0.0867 \\
\hline 8.9 & 0.5486 & 0.7396 & 1.1595 & 1.4663 & 1.7135 & 1.6639 & 1.5567 & 1.4319 & 1.1888 & 0.9268 & 0.5999 & 0.4824 \\
\hline 9.10 & 1.1723 & 1.3931 & 1.9254 & 2.2583 & 2.4680 & 2.3466 & 2.1984 & 2.1187 & 1.9171 & 1.6743 & 1.2477 & 1.0696 \\
\hline $10-11$ & 1.7279 & 1.9355 & 2.5460 & 2.8754 & 3.0602 & 2.8773 & 2.6969 & 2.6530 & 2.4932 & 2.2977 & 1.8148 & 1.6027 \\
\hline $11-12$ & 2.0874 & 2.2820 & 2,9326 & 3.2630 & 3.4267 & 3.2034 & 3.0006 & 2.9827 & 2.8509 & 2.6942 & 2.1788 & 1.9509 \\
\hline $12 \cdot 13$ & 2.1877 & 2.3725 & 3.0418 & 3.3787 & 3.5390 & 3,3031 & 3.0887 & 3.0760 & 2.9542 & 2.8093 & 2.2849 & 2.0451 \\
\hline $13-14$ & 2.0203 & 2.2195 & 2.8668 & 3.2129 & 3,3844 & 3.1644 & 2.9514 & 2.9271 & 2.7933 & 2.6315 & 2.1172 & 1.8831 \\
\hline 14.15 & $\frac{.0200}{1.6220}$ & 1.8303 & 2.4333 & 2.7906 & 2.9868 & 2.8089 & 2.6099 & 2.5573 & 2.3939 & 2.1943 & 1.7139 & 1.4944 \\
\hline $15-16$ & 1.0594 & 1.2770 & 1.8064 & 2.1661 & 2.3943 & 2.2771 & 2.1029 & 2.0129 & 1.8100 & 1.5653 & 1.1426 & 0.9576 \\
\hline $16-17$ & 0.4525 & 0.6531 & 1.0795 & 1.4162 & 1.6683 & 1.6234 & 1.4821 & 1.3508 & 1.1232 & 0.8436 & 0.5186 & 0.3781 \\
\hline $17-18$ & 0.0000 & 0.0000 & 0.3585 & 0.6519 & 0.8922 & 0.9122 & 0.8137 & 0.6638 & 0.4332 & 0.1828 & 0.0000 & 0.0000 \\
\hline \multirow[t]{2}{*}{$18-19$} & 0.0000 & 0.0000 & 0.0000 & 0.0000 & 0.2190 & 0.2747 & 0.2236 & 0.0904 & 0.0000 & 0.0000 & 0.0000 & 0.0000 \\
\hline & 12.9913 & 14.9188 & 20.6843 & 24.3156 & 26.9810 & 25.7742 & 23.9539 & 22.7942 & 20.5622 & 18.1089 & 13.7526 & 11.9506 \\
\hline & & 00010 & 00081 & 01126 & 01142 & 01101 & 0,0996 & 0.0992 & 0.0985 & 0.0906 & 0.0762 & 0.0660 \\
\hline \begin{tabular}{|l} 
Peak kW \\
demand credit $Q$
\end{tabular} & $\frac{0.0706}{\$ 0.0265}$ & \begin{tabular}{r|}
0.0849 \\
$\$ 0.0318$
\end{tabular} & $\frac{0.0987}{\$ 0.0368}$ & $\begin{array}{r}0.1120 \\
\$ 0.0422\end{array}$ & $\$ 0.0428$ & $\$ 0.0455$ & $\$ 0.0412$ & $\$ 0.0410$ & \begin{tabular}{r|}
$\$ .00007$ \\
$\$ 0.0407$
\end{tabular} & $\$ 0.0374$ & $\$ 0.0286$ & $\frac{0.0000}{\$ 0.0247}$ \\
\hline \multicolumn{13}{|l|}{$20 \%$} \\
\hline Total energy charges/yr & $\$ 20.7956$ & & & & & & & & & & & \\
\hline Total demand charges/yr & $\$ 0.4392$ & & & & & & & & & & & \\
\hline TotalkWh/m2/yr & 236.79 & & & & & & & & & & & \\
\hline Av. \$ $\mathrm{kWh}$ & $\$ 0.0897$ & & & & & & & & & & & \\
\hline & & & & & & & & & & & & \\
\hline
\end{tabular}




\begin{tabular}{|c|c|c|c|c|c|c|c|c|c|c|c|c|}
\hline UTILITY RATES & MIAMI & & & & & & & & & & & \\
\hline \multicolumn{13}{|l|}{ Florlda Power \& Light } \\
\hline Rate GSDT-1 & Jan & Feb & Mar & Apr & May & Jun & Jul & Aug & Sep & Oct & Nov & Dec \\
\hline $5-6$ & 0.0470 & 0.0470 & 0.0470 & 0.0309 & 0.0309 & 0.0309 & 0.0309 & 0.0309 & 0.0309 & 0.0309 & 0.0470 & 0.0470 \\
\hline $6-7$ & 0.0747 & 0.0747 & 0.0747 & 0.0309 & 0.0309 & 0.0309 & 0.0309 & 0.0309 & 0.0309 & 0.0309 & 0.0747 & 0.0747 \\
\hline $7-8$ & 0.0747 & 0.0747 & 0.0747 & 0.0309 & 0.0309 & 0.0309 & 0.0309 & 0.0309 & 0.0309 & 0.0309 & 0.0747 & 0.0747 \\
\hline $8-9$ & 0.0747 & 0.0747 & 0.0747 & 0.0309 & 0.0309 & 0.0309 & 0.0309 & 0.0309 & 0.0309 & 0.0309 & 0.0747 & 0.0747 \\
\hline $9 \cdot 10$ & 0.0747 & 0.0747 & 0.0747 & 0.0309 & 0.0309 & 0.0309 & 0.0309 & 0.0309 & 0.0309 & 0.0309 & 0.0747 & 0.0747 \\
\hline $10-11$ & 0.0470 & 0.0470 & 0.0470 & 0.0309 & 0.0309 & 0.0309 & 0.0309 & 0.0309 & 0.0309 & 0.0309 & 0.0470 & 0.0470 \\
\hline $11-12$ & 0.0470 & 0.0470 & 0.0470 & 0.0309 & 0.0309 & 0.0309 & 0.0309 & 0.0309 & 0.0309 & 0.0309 & 0.0470 & 0.0470 \\
\hline $12 \cdot 13$ & 0.0470 & 0.0470 & 0.0470 & 0.0585 & 0.0585 & 0.0585 & 0.0585 & 0.0585 & 0.0585 & 0.0585 & 0.0470 & 0.0470 \\
\hline 13-14 & 0.0470 & 0.0470 & 0.0470 & 0.0585 & 0.0585 & 0.0585 & 0.0585 & 0.0585 & 0.0585 & 0.0585 & 0.0470 & 0.0470 \\
\hline $14-15$ & 0.0470 & 0.0470 & 0.0470 & 0.0585 & 0.0585 & 0.0585 & 0.0585 & 0.0585 & 0.0585 & 0.0585 & 0.0470 & 0.0470 \\
\hline $15-16$ & 0.0470 & 0.0470 & 0.0470 & 0.0585 & 0.0585 & 0.0585 & 0.0585 & 0.0585 & 0.0585 & 0.0585 & 0.0470 & 0.0470 \\
\hline $16-17$ & 0.0470 & 0.0470 & 0.0470 & 0.0585 & 0.0585 & 0.0585 & 0.0585 & 0.0585 & 0.0585 & 0.0585 & 0.0470 & 0.0470 \\
\hline $17-18$ & 0.0470 & 0.0470 & 0.0470 & 0.0585 & 0.0585 & 0.0585 & 0.0585 & 0.0585 & 0.0585 & 0.0585 & 0.0470 & 0.0470 \\
\hline $18-19$ & 0.0747 & 0.0747 & 0.0747 & 0.0585 & 0.0585 & 0.0585 & 0.0585 & 0.0585 & 0.0585 & 0.0585 & 0.0747 & 0.0747 \\
\hline & & & & & & & & & & & & \\
\hline \multicolumn{13}{|l|}{ PV OUTPUT } \\
\hline \multicolumn{13}{|l|}{ PER HOUR } \\
\hline & & & & & & & & & & & & \\
\hline 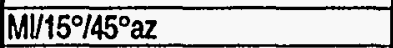 & Jan & Feb & Mar & Apr & May & Jun & Jul & Aug & $\operatorname{sep}$ & Oct & Nov & Dec \\
\hline 5.6 & 0.0000 & 0.0000 & 0.0000 & 0.0000 & 0.0290 & 0.0550 & 0.0429 & 0.0000 & 0.0000 & 0.0000 & 0.0000 & 0.0000 \\
\hline 6.7 & 0.0000 & 0.0152 & 0.1027 & 0.1757 & 0.2443 & 0.2559 & 0.2528 & 0.1995 & 0.1215 & 0.0392 & 0.0000 & 0.0000 \\
\hline $7-8$ & 0.1924 & 0.2685 & 0.4432 & 0.5585 & 0.6463 & 0.6162 & 0.6356 & 0.5540 & 0.4287 & 0.3197 & 0.2082 & 0.1688 \\
\hline 8.9 & 0.6437 & 0.7549 & 1.0392 & 1.1704 & 1.2108 & 1.0934 & 1.1560 & 1.0692 & 0.9166 & 0.8251 & 0.6624 & 0.6054 \\
\hline $9 \cdot 10$ & 1.1803 & 1.2816 & 1.6522 & 1.7589 & 1.7291 & 1.5268 & 1.6306 & 1.5548 & 1.4047 & 1.3618 & 1.1844 & 1.1407 \\
\hline 10.11 & 1.6240 & 1.7134 & 2.1327 & 2.2159 & 2.1322 & 1.8640 & 2.0003 & 1.9325 & 1.7842 & 1.7968 & 1.6163 & 1.5892 \\
\hline $11-12$ & 1.9009 & 1.9786 & 2.4258 & 2.4930 & 2.3740 & 2.0653 & 2.2212 & 2.1587 & 2.0140 & 2.0615 & 1.8847 & 1.8657 \\
\hline $12 \cdot 13$ & 1.9669 & 2.0440 & 2.4978 & 2.5620 & 2.4326 & 2.1117 & 2.2731 & 2.2111 & 2.0662 & 2.1233 & 1.9492 & 1.9350 \\
\hline 13.14 & 1.8180 & 1.9035 & 2,3429 & 2.4172 & 2.3026 & 1.9997 & 2.1519 & 2.0842 & 1.9370 & 1.9787 & 1.8049 & 1.7862 \\
\hline $14-15$ & 1.4771 & 1.5787 & 1.9838 & 2.0801 & 2.0025 & 1.7443 & 1.8737 & 1.7967 & 1.6450 & 1.6484 & 1.4752 & 1.4433 \\
\hline $15-16$ & 1.0020 & 1.1217 & 1.4686 & 1.5927 & 1.5686 & 1.3761 & 1.4732 & 1.3844 & 1.2276 & 1.1827 & 1.0135 & 0.9675 \\
\hline 16-17 & 0.4769 & 0.5985 & 0.8727 & 1.0138 & 1.0477 & 0.9345 & 0.9922 & 0.8955 & 0.7441 & 0.6500 & 0.4984 & 0.4456 \\
\hline $17-18$ & 0.0000 & 0.1173 & 0.2866 & 0.4340 & 0.5136 & 0.4799 & 0.4980 & 0.4042 & 0.2688 & 0.1503 & 0.0000 & 0.0000 \\
\hline \multirow[t]{2}{*}{$18-19$} & 0.0000 & 0.0000 & 0.0000 & 0.0000 & 0.0678 & 0.0938 & 0.0816 & 0.0000 & 0.0000 & 0.0000 & 0.0000 & 0.0000 \\
\hline & 12.2822 & 13.3759 & 17.2482 & 18.4722 & 18.3011 & 16.2166 & 17.2831 & 16.2448 & 14.5584 & 14.1384 & 12.2972 & 11.9474 \\
\hline & & & & & & & & & & & & \\
\hline Peak kW & 0.0634 & 0.0730 & 0.0806 & 0.0854 & 0.0785 & 0.0704 & 0.0733 & 0.0713 & 0.0689 & 0.0685 & 0.0650 & 0.0624 \\
\hline demand credil 6 & $\$ 0.0883$ & $\$ 0.1016$ & $\$ 0.1122$ & $\$ 0.1189$ & $\$ 0.1093$ & $\$ 0.0980$ & $\$ 0.1021$ & $\$ 0.0993$ & $\$ 0.0959$ & $\$ 0.0954$ & $\$ 0.0905$ & $\$ 0.0869$ \\
\hline \multicolumn{13}{|l|}{$20 \%$} \\
\hline Total energy charges/yr & $\$ 19.9043$ & & & & & & & & & & & \\
\hline Total demand charges/yr & $\$ 1.1983$ & & & & & & & & & & & \\
\hline Total kWh/m2/yr & 182.37 & & & & & & & & & & & \\
\hline Av. SkWh & $\$ 0.1157$ & & & & & & & & & & & \\
\hline & & & & & & & & & & & & \\
\hline
\end{tabular}




\begin{tabular}{|c|c|c|c|c|c|c|c|c|c|c|c|c|}
\hline \multirow{2}{*}{\begin{tabular}{|l} 
UTILITY RATES \\
Commonwealth Edison \\
\end{tabular}} & \multicolumn{12}{|l|}{ CHICAGO } \\
\hline & \multicolumn{2}{|c|}{ (includes ECA) } & \multirow[b]{2}{*}{ Mar } & \multirow[b]{2}{*}{ Apr } & \multirow[b]{2}{*}{ May } & \multirow[b]{2}{*}{ Jun } & \multirow[b]{2}{*}{ Jul } & \multirow[b]{2}{*}{ Aug } & \multirow[b]{2}{*}{ Sep } & \multirow[b]{2}{*}{ Oet } & \multirow[b]{2}{*}{ Nov } & \multirow[b]{2}{*}{ Dec } \\
\hline Rate Sched 6 TOU & Jan & Feb & & & & & & & & & & \\
\hline 56 & 0.0296 & 0.0296 & 0.0296 & 0.0296 & 0.0296 & 0.0284 & 0.0284 & 0.0284 & 0.0284 & 0.0296 & 0.0296 & 0.0296 \\
\hline $6-7$ & 0.0296 & 0.0296 & 0.0296 & 0.0296 & 0.0296 & 0.0284 & 0.0284 & 0.0284 & 0.0284 & 0.0296 & 0.0296 & 0.0296 \\
\hline $7-8$ & 0.0296 & 0.0296 & 0.0296 & 0.0296 & 0.0296 & 0.0284 & 0.0284 & 0.0284 & 0.0284 & 0.0296 & 0.0296 & 0.0296 \\
\hline $8-9$ & 0.0296 & 0.0296 & 0.0296 & 0.0296 & 0.0296 & 0.0284 & 0.0284 & 0.0284 & 0.0284 & 0.0296 & 0.0296 & 0.0296 \\
\hline $9-10$ & 0.0644 & 0.0644 & 0.0644 & 0.0644 & 0.0644 & 0.0631 & 0.0631 & 0.0631 & 0.0631 & 0.0644 & 0.0644 & 0.0644 \\
\hline $10-11$ & 0.0644 & 0.0644 & 0.0644 & 0.0644 & 0.0644 & 0.0631 & 0.0631 & 0.0631 & 0.0631 & 0.0644 & 0.0644 & 0.0644 \\
\hline 11.12 & 0.0644 & 0.0644 & 0.0644 & 0.0644 & 0.0644 & 0.0531 & 0.0631 & 0.0631 & 0.0631 & 0.0644 & 0.0644 & 0.0644 \\
\hline $12-13$ & 0.0644 & 0.0644 & 0.0644 & 0.0644 & 0.0644 & 0.0631 & 0.0631 & 0.0631 & 0.0631 & 0.0644 & 0.0644 & 0.0644 \\
\hline 13.14 & 0.0644 & 0.0644 & 0.0644 & 0.0644 & 0.0644 & 0.0631 & 0.0631 & 0.0631 & 0.0631 & 0.0644 & 0.0644 & 0.0644 \\
\hline $14 \cdot 15$ & 0.0644 & 0.0644 & 0.0644 & 0.0644 & 0.0644 & 0.0631 & 0.0631 & 0.0631 & 0.0631 & 0.0644 & 0.0644 & 0.0644 \\
\hline $15-16$ & 0.0644 & 0.0644 & 0.0644 & 0.0644 & 0.0644 & 0.0631 & 0.0631 & 0.0631 & 0.0631 & 0.0644 & 0.0644 & 0.0644 \\
\hline $16-17$ & 0.0644 & 0.0644 & 0.0644 & 0.0644 & 0.0644 & 0.0631 & 0.0631 & 0.0631 & 0.0631 & 0.0644 & 0.0644 & 0.0644 \\
\hline $17 \cdot 18$ & 0.0644 & 0.0644 & 0.0644 & 0.0644 & 0.0644 & 0.0631 & 0.0631 & 0.0631 & 0.0631 & 0.0644 & 0.0644 & 0.0644 \\
\hline $18-19$ & 0.0644 & 0.0644 & 0.0644 & 0.0644 & 0.0644 & 0.0631 & 0.0631 & 0.0631 & 0.0631 & 0.0644 & 0.0644 & 0.0644 \\
\hline & & & & & & & & & & & & \\
\hline \multicolumn{13}{|l|}{ PV OUTPUT (Option 1, PVI) } \\
\hline \multicolumn{13}{|l|}{ PER HOUR } \\
\hline & & & & & & & & & & & & \\
\hline ChV15\% $/ 45^{\circ}$ az & Jan & Feb & Mar & Apr & May & Jun & Jul & Aug & Sep & Oct & Nov & Dec \\
\hline $5 \cdot 6$ & 0.0000 & 0.0000 & 0.0000 & 0.0079 & 0.1244 & 0.1616 & 0.1435 & 0.0538 & 0.0000 & 0.0000 & 0.0000 & 0.0000 \\
\hline $6-7$ & 0.0000 & 0.0000 & 0.0773 & 0.1990 & 0.3370 & 0.3874 & 0.3620 & 0.2457 & 0.1173 & 0.0000 & 0.0000 & 0.0000 \\
\hline$\overline{7-8}$ & 0.0291 & 0.1276 & 0.3280 & 0.5280 & 0.7615 & 0.8392 & 0.8098 & 0.6315 & 0.3913 & 0.1840 & 0.0527 & 0.0022 \\
\hline 8.9 & 0.2639 & 0.4271 & 0.7799 & 1.0164 & 1.2835 & 1.3494 & 1.3382 & 1.1713 & 0.8705 & 0.5770 & 0.2933 & 0.1982 \\
\hline $9 \cdot 10$ & 0.6201 & 0.8246 & 1.2665 & 1.4970 & 1.7559 & 1.8117 & 1.8154 & 1.6809 & 1.3729 & 1.0821 & 0.6453 & 0.4842 \\
\hline $10-11$ & 0.9574 & 1.1772 & 1.6706 & 1.8751 & 2.1247 & 2.1689 & 2.1856 & 2.0766 & 1.7811 & 1.5177 & 0.9760 & 0.7624 \\
\hline 11.12 & 1.1810 & 1.4041 & 1.9238 & 2.1079 & 2.3500 & 2.3862 & 2.4114 & 2.3208 & 2.0350 & 1.7952 & 1.1879 & 0.9488 \\
\hline $12-13$ & 1.2424 & 1.4634 & 1.9905 & 2.1694 & 2.4107 & 2.4464 & 2.4733 & 2.3879 & 2.1053 & 1.8745 & 1.2479 & 0.9947 \\
\hline 13.14 & 1.1255 & 1.3498 & 1.8644 & 2.0550 & 2.3030 & 2.3457 & 2.3681 & 2.2733 & 1.9849 & 1.7426 & 1.1365 & 0.8923 \\
\hline $14-15$ & 0.8588 & 1.0868 & 1.5674 & 1.7813 & 2.0413 & 2.0971 & 2.1094 & 1.9931 & 1.6923 & 1.4268 & 0.8784 & 0.6663 \\
\hline $15-16$ & 0.4984 & 0.7178 & 1.1442 & 1.3834 & 1.6560 & 1.7281 & 1.7263 & 1.5814 & 1.2715 & 0.9803 & 0.5314 & 0.3634 \\
\hline $16-17$ & 0.1338 & 0.3205 & 0.6569 & 0.9138 & 1.1896 & 1.2780 & 1.2609 & 1.0860 & 0.7836 & 0.4860 & 0.1724 & 0.0651 \\
\hline $17-18$ & 0.0000 & 0.0000 & 0.2012 & 0.4400 & 0.6914 & 0.7870 & 0.7550 & 0.5715 & 0.3007 & 0.0000 & 0.0000 & 0.0000 \\
\hline \multirow[t]{2}{*}{$18-19$} & 0.0000 & 0.0000 & 0.0000 & 0.0489 & 0.2402 & 0.3241 & 0.2874 & 0.1300 & 0.0000 & 0.0000 & 0.0000 & 0.0000 \\
\hline & 6.9104 & 8.8989 & 13.4727 & 16.0231 & 19.2692 & 20.1108 & 20.0463 & 18.2041 & 14.7064 & 11.6662 & 7.1218 & 5.3776 \\
\hline \multirow[b]{2}{*}{ PeakkW } & & & & & & & & & & & & \\
\hline & 0.0401 & 0.0523 & 0.0842 & 0.0723 & 0.0778 & 0.0815 & 0.0798 & 0.0770 & 0.0702 & 0.0605 & 0.0416 & 0.0321 \\
\hline demand credit 0 & $\$ 0.0951$ & $\$ 0.1241$ & $\$ 0.1524$ & $\$ 0.1717$ & $\$ 0.1846$ & $\$ 0.2476$ & $\$ 0.2422$ & $\$ 0.2338$ & $\$ 0.2130$ & $\$ 0.1435$ & $\$ 0.0987$ & $\$ 0.0762$ \\
\hline \multicolumn{13}{|l|}{$20 \%$} \\
\hline Total energy charges/yr & $\$ 9.7554$ & & & & & & & & & & & \\
\hline Total demand charges/yr & $\$ 1.9831$ & & & & & & & & & & & \\
\hline Total kWh/m2/yr & 161.81 & & & & & & & & & & & \\
\hline Av. SWWh & $\$ 0.0725$ & & & & & & & & & & & \\
\hline & & & & & & & & & & & & \\
\hline
\end{tabular}




\begin{tabular}{|c|c|c|c|c|c|c|c|c|c|c|c|c|}
\hline \multirow{2}{*}{$\begin{array}{l}\text { UTILITY RATES } \\
\text { Cincinnall Gas \& Electric }\end{array}$} & \multicolumn{2}{|c|}{ CINCINNATI } & & & & & & & & & & \multirow{3}{*}{ Dec } \\
\hline & & & & & & & \multirow[b]{2}{*}{ Jul } & \multirow[b]{2}{*}{ Aun } & \multirow[b]{2}{*}{ Sep } & \multirow[b]{2}{*}{ loct } & \multirow[b]{2}{*}{ Nov } & \\
\hline $\begin{array}{l}\text { Rate Comm, Gen. Service } \\
\text { Schedule DS }\end{array}$ & Jan & Feb & Mar & Apr & May & Jun & & & & & & Dec \\
\hline $5-6$ & 0.0469284 & 0.0469284 & 0.0469284 & 0.0469284 & 0.0469284 & 0.0470654 & 0.0470654 & 0.0470654 & 0.0470654 & 0.0469284 & 0.0469284 & \\
\hline $6-7$ & 0.0469284 & 0.0469284 & 0.0469284 & 0.0469284 & 0.0469284 & 0.0470654 & 0.0470654 & 0.0470654 & 0.0470654 & 0.0469284 & 0.0469284 & 0.0469284 \\
\hline 7.8 & 0.0469284 & 0.0469284 & 0.0469284 & 0.0469284 & 0.0469284 & 0.0470654 & 0.0470654 & 0.0470654 & 0.0470654 & 0.0469284 & $0: 0469284$ & 0.0469284 \\
\hline 8.9 & 0.0469284 & 0.0469284 & 0.0469284 & 0.0469284 & 0.0469284 & 0.0470654 & 0.0470654 & 0.0470654 & 0.0470654 & 0.0469284 & 0.0469284 & 0.0469284 \\
\hline 9.10 & 0.0469284 & 0.0469284 & 0.0469284 & 0.0469284 & 0.0469284 & 0.0470654 & 0.0470654 & 0.0470654 & 0.0470654 & 0.0469284 & 0.0469284 & 0.0469284 \\
\hline $10-11$ & 0.0469284 & 0.0469284 & 0.0469284 & 0.0469284 & 0.0469284 & 0.0470654 & 0.0470654 & 0.0470654 & 0.0470654 & 0.0469284 & 0.0469284 & 0.0469284 \\
\hline $11-12$ & 0.0469284 & 0.0469284 & 0.0469284 & 0.0469284 & 0.0469284 & 0.0470654 & 0.0470654 & 0.0470654 & 0.0470654 & 0.0469284 & 0.0469284 & 0.0469284 \\
\hline $12-13$ & 0.0469284 & 0.0469284 & 0.0469284 & 0.0469284 & 0.0469284 & 0.0470654 & 0.0470654 & 0.0470654 & 0.0470654 & 0.0469284 & 0.0469284 & 0.0469284 \\
\hline $13-14$ & 0.0469284 & 0.0469284 & 0.0469284 & 0.0469284 & 0.0469284 & 0.0470654 & 0.0470654 & 0.0470654 & 0.0470654 & 0.0469284 & 0.0469284 & 0.0469284 \\
\hline 14.15 & 0.0469284 & 0.0469284 & 0.0469284 & 0.0469284 & 0.0469284 & 0.0470654 & 0.0470654 & 0.0470654 & 0.0470654 & 0.0469284 & 0.0469284 & 0.0469284 \\
\hline $15-16$ & 0.0469284 & 0.0469284 & 0.0469284 & 0.0469284 & 0.0469284 & 0.0470654 & 0.0470654 & 0.0470654 & 0.0470654 & 0.0469284 & 0,0469284 & 0.0469284 \\
\hline $16-17$ & 0.0469284 & 0.0469284 & 0.0469284 & 0.0469284 & 0.0469284 & 0.0470654 & 0.0470654 & \begin{tabular}{|l}
0.0470654 \\
\end{tabular} & 0.0470654 & 0.0469284 & 0.0469284 & 0.0469284 \\
\hline $17-18$ & 0.0469284 & 0.0469284 & 0.0469284 & 0.0469284 & 0.0469284 & 0.0470654 & 0.0470654 & 0.0470654 & 0.0470654 & 0.0469284 & 0.0469284 & 0.0469284 \\
\hline $18-19$ & 0.0469284 & 0.0469284 & 0.0469284 & 0.0469284 & 0.0469284 & 0.0470654 & 0.0470654 & 0.0470654 & 0.0470654 & 0.0469284 & 0.0469284 & 0.0469284 \\
\hline & & & & & & & & & & & & \\
\hline \multicolumn{13}{|l|}{ PV OUTPUT (Optlon 1, PV1) } \\
\hline \multicolumn{13}{|l|}{ PER HOUR } \\
\hline & & & & & & & & & & & & \\
\hline $\mathrm{C} \ln / 15^{\circ} / 45^{\circ} \mathrm{az}$ & Jan & Feb & Mar & Apr & May & Jun & Jul & Aug & Sep & Oct & $\mathrm{Nov}$ & $\overline{\text { Dec }}$ \\
\hline 56 & 0,0000 & 0,0000 & 0.0000 & 0.0000 & 0.1063 & 0.1423 & 0.1249 & 0.0414 & 0.0000 & 0.0000 & 0.0000 & 0.0000 \\
\hline $6-7$ & 0.0000 & 0.0000 & 0.0788 & 0.1925 & 0.3146 & 0.3556 & 0.3350 & 0.2372 & 0.1176 & 0.0000 & 0.0000 & 0.0000 \\
\hline $7-8$ & 0.0512 & 0.1425 & 0.3260 & 0.5098 & 0.7085 & 0.7654 & 0.7403 & 0.6054 & 0.3923 & 0.2055 & 0.0735 & 0.0260 \\
\hline $8-9$ & 0.2866 & 0.4337 & 0.7340 & 0.9722 & 1.1958 & 1.2371 & 1.2243 & 1.1174 & 0.8503 & 0.6104 & 0.3255 & 0.2360 \\
\hline 9.10 & 0.6094 & 0.7967 & 1.1658 & 1.4252 & 1.6380 & 1.6644 & 1.6621 & 1.5988 & 1.3251 & 1.1073 & 0.6700 & 0.5261 \\
\hline $10-11$ & 0.9077 & 1.1104 & 1.5209 & 1.7785 & 1.9831 & 1.9948 & 2.0019 & 1.9734 & 1.7079 & 1.5263 & 0.9836 & 0.7954 \\
\hline $11-12$ & 1.0976 & 1.3079 & 1.7402 & 1.9952 & 2.1923 & 2.1938 & 2.2071 & 2.2025 & 1.9433 & 1.7881 & 1.1857 & 0.9689 \\
\hline $12 \cdot 13$ & 1.1448 & 1.3569 & 1.7940 & 2.0495 & 2.2451 & 2.2458 & 2.2599 & 2.2619 & 2.0039 & 1.8589 & 1.2339 & 1.0112 \\
\hline $13-14$ & 1.0367 & 1.2487 & 1.6748 & 1.9366 & 2.1384 & 2.1461 & 2.1564 & 2.1475 & 1.8850 & 1.7277 & 1.1247 & 0.9087 \\
\hline $14 \cdot 15$ & 0.7939 & 1.0051 & 1.4034 & 1.6731 & 1.8861 & 1.9083 & 1.9105 & 1.8752 & 1.6038 & 1.4163 & 0.8769 & 0.6854 \\
\hline $15-16$ & 0.4721 & 0.6684 & 1.0211 & 1.2922 & 1.5181 & 1.5588 & 1.5500 & 1.4786 & 1.2012 & 0.9795 & 0.5433 & 0.3895 \\
\hline $16-17$ & 0.1420 & 0.3048 & 0.5867 & 0.8451 & 1.0755 & 1.1364 & 1.1150 & 1.0034 & 0.7359 & 0.4936 & 0.1932 & 0.0926 \\
\hline $17-18$ & 0.0000 & 0.0000 & 0.1760 & 0.3968 & 0.6077 & 0.6793 & 0.6489 & 0.5141 & 0.2777 & 0.0000 & 0.0000 & 0.0000 \\
\hline $18-19$ & 0.0000 & 0.0000 & 0.0000 & 0.0000 & 0.1916 & 0.2586 & 0.2267 & 0.0997 & 0.0000 & 0.0000 & 0.0000 & 0.0000 \\
\hline & 6.5420 & 8.3751 & 12.2217 & 15.0667 & 17.8011 & 18.2867 & 18.1630 & 17.1565 & 14.0440 & 11.7136 & 7.2103 & 5.6398 \\
\hline & & & & & & & & & & & & \\
\hline PeakkW & 0.0369 & 0.0485 & 0.0579 & 0.0683 & 0.0724 & 0.0749 & 0.0729 & 0.0730 & 0.0668 & 0.0600 & 0.0411 & 0.0326 \\
\hline demand credil Q & $\$ 0.0717$ & $\$ 0.0941$ & $\$ 0.1124$ & $\$ 0.1327$ & $\$ 0.1406$ & $\$ 0.1454$ & $\$ 0.1416$ & $\$ 0.1417$ & $\$ 0.1297$ & $\$ 0.1165$ & $\$ 0.0799$ & $\$ 0.0633$ \\
\hline $20 \%$ & & & & & & & & & & & & \\
\hline Total energy charges/yr & $\$ 7.1527$ & & & & & & & & & & & \\
\hline Total demand charges/yr & $\$ 1.3696$ & & & & & & & & & & & \\
\hline Total kWh/m2/yr & 152.22 & & & & & & & & & & & \\
\hline Av. S/kWh & $\$ 0.0560$ & & & & & & & & & & & \\
\hline & & & & & & & & & & & & \\
\hline
\end{tabular}




\section{REPORT DOCUMENTATION PAGE}

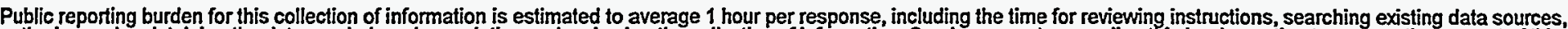

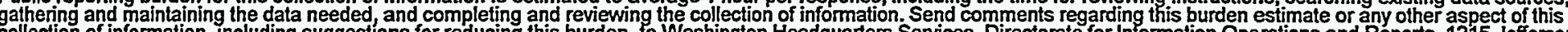

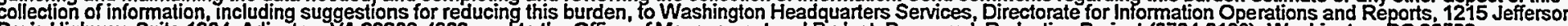

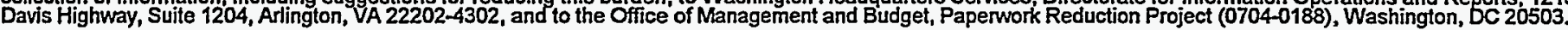

\begin{tabular}{|l|l|l}
\hline 1. AGENCY USE ONLY (Leave blank) & $\begin{array}{c}\text { 2. REPORT DATE } \\
\text { March } 1995\end{array}$ & $\begin{array}{c}\text { 3. REPORT TYPE AND DATES COVERED } \\
\text { Subcontract Report }\end{array}$ \\
\hline
\end{tabular}

\section{TITLE AND SUBTITLE}

5. FUNDING NUMBERS

Building Integrated Photovoitaics: A Case Study

(C) AAE-5-14456-01

(TA) BE 51.2101

\section{AUTHOR(S)}

Gregory Kiss, Jennifer Kinkead, Mahadev Raman

\section{PERFORMING ORGANIZATION NAME(S) AND ADDRESS(ES)}

Kiss Cathcart Anders Architects, P.C.

150 Nassau St.

New York, NY 10038

8. PERFORMING ORGANIZATION REPORT NUMBER

NA

\section{SPONSORING/MONITORING AGENCY NAME(S) AND ADDRESS(ES)}

National Renewable Energy Laboratory

1617 Cole Boulevard

Golden, CO 80401-3393

10. SPONSORING/MONITORING AGENCY REPORT NUMBER

NRELTP-472-7574

\section{SUPPLEMENTARY NOTES}

\section{2a, DISTRIBUTION/AVAILABILITY STATEMENT}

National Technical Information Service

U.S, Department of Commerce

5285 Port Royal Road

Springfield, VA 22161 12b. DISTRIBUTION CODE

UC-1600

\section{ABSTRACT (Maximum 200 words)}

In 1992, Kiss Cathcart Anders Architects performed a study for NREL on building integrated photovoltaic (BIPV) issues as seen from the perspective of the building community. That study included a broad overview of potential BIPV applications in commercial /institutional buildings. In general, the purpose of the study was to list major issues and potential applications; by its nature it asked more questions than it answered.

The second phase of the studyreported on here-was commissioned by NREL in 1994 to produce quantitative data on the performance of specific BIPV systems. High value-added applications for BIPV are targeted in medium to high-end commercial/institutional buildings. These building types should offer a good initial market for BIPV products. The study did not attempt to identify the best possible application for BIPV. The study reached the following conclusions: (1) Payback periods range from 14.5 years and up. (2) Systems with payback periods of approximately 20 years can be cost effective today. (3) No single PV technology is most cost effective in all cases. (4) No single BIPV roof system is most cost effective in all cases.

\section{SUBJECT TERMS}

Building-integrated photovoltaics, roof-integrated photovoltaics
15. NUMBER OF PAGES

136

16. PRICE CODE

$\mathrm{A} 07$

20. LIMITATION OF ABSTRACT
18. SECURITY CLASSIFICATION OF THIS PAGE
19. SECURITY CLASSIFICATION OF ABSTRACT

\section{SECURITY CLASSIFICATION} OF REPORT
Standard Form 298 (Rev. 2-89) Prescribed by ANSI Std. Z39-18
NSN 7540-01-280-5500 